\title{
Short- and intermediate-term health outcomes following Hypertensive Disorders in Pregnancy in Nigeria
}

Gezondheidsuitkomsten op de korte en middellange termijn van vrouwen met een hypertensieve aandoening tijdens de zwangerschap 
Colophon

Print: Ridderprint | www.ridderprint.nl 


\title{
Short- and intermediate-term health outcomes following Hypertensive Disorders in Pregnancy in Nigeria
}

\author{
Gezondheidsuitkomsten op de korte en middellange termijn van vrouwen \\ met een hypertensieve aandoening tijdens de zwangerschap
}

(met een samenvatting in het Nederlands)

\author{
Proefschrift \\ ter verkrijging van de graad van doctor aan de \\ Universiteit Utrecht \\ op gezag van de \\ rector magnificus, prof.dr. H.R.B.M. Kummeling, \\ ingevolge het besluit van het college voor promoties \\ in het openbaar te verdedigen op
}

donderdag 25 maart 2021 des middags te 12.45 uur

door

Ishaku Mohammed Salisu

geboren op 22 januari 1972

te Takum, Nigeria 


\section{Promotoren:}

Prof. dr. D.E. Grobbee

Prof. dr. A. Franx

\section{Copromotoren:}

Dr. J.L. Browne

Dr. A.G. Kayode

Dit proefschrift werd (mede) mogelijk gemaakt met financiële steun van de Population Council en de US Agency for International Development (USAID, APSOAA-14-000048). 
Chapter 1

PART 1

Chapter 2

Chapter 3

Chapter 4

Chapter 5

Chapter 6
General Introduction

9

Quality of care for women with hypertensive disorders in pregnancy

19

Detecting and managing hypertensive disorders in pregnancy: a cross-

sectional analysis of the quality of antenatal care in Nigeria

Adherence to Guidelines in Postpartum Management of Hypertensive

Disorders in Pregnancy in Nigeria: A Multi-centre Study

Chronic medical disorders associated with prior hypertensive disorders in

Pregnancy

Persistent Hypertension up to One Year Postpartum among Women with Hypertensive Disorders in Pregnancy in a Low-resource Setting: a Prospective Cohort Study

Metabolic syndrome following hypertensive disorders in pregnancy in a lowresource setting: A cohort study

Prevalence and determinants of chronic kidney disease in women with hypertensive disorders in pregnancy in Nigeria: a cohort study

Community-based approach to management of hypertensive disorders in Pregnancy 161 
Chapter $7 \quad$ Exploring survivor perceptions of pre-eclampsia and eclampsia in Nigeria $\begin{array}{ll}\text { through the health belief model } & 163\end{array}$

Chapter 8 A primary health care model for managing pre-eclampsia and eclampsia in low-

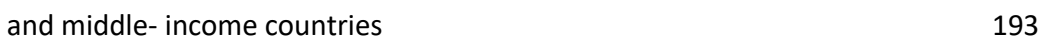

$\begin{array}{llr}\text { Chapter } 9 & \text { General discussion } & 210\end{array}$

$\begin{array}{llr}\text { Appendix } & \text { Summary } & 220\end{array}$

$\begin{array}{ll}\text { Samenvatting } & 224\end{array}$

$\begin{array}{lr}\text { Contributing authors } & 228\end{array}$

$\begin{array}{lr}\text { Acknowledgements } & 231\end{array}$

$\begin{array}{ll}\text { Curriculum vitae } & 233\end{array}$

$\begin{array}{ll}\text { Lists of publications } & 236\end{array}$ 




\section{Chapter 1}

\section{General introduction}

\section{Background}

Hypertensive disorders in pregnancy (HDP) is a group of medical conditions during pregnancy generally characterized by the presence of hypertension (defined as systolic blood pressure of $\geq 140 \mathrm{mmHg}$ and/or diastolic blood pressure of $\geq 90 \mathrm{mmHg}$ measured on two consecutive periods 4-6 hours apart) ${ }^{1-2}$. As classified by the International Society for the Study of Hypertension in pregnancy (ISSHP), HDP includes chronic hypertension, gestational hypertension and pre-eclampsia ${ }^{1-2}$. Chronic hypertension is hypertension diagnosed before, or within the first 20 weeks of pregnancy. Gestational hypertension occurs for the first time in pregnancy after 20 weeks of gestation without proteinuria or other biochemical or hematological abnormalities while pre-eclampsia is gestational hypertension associated with excess protein in urine $(>300 \mathrm{mg} / \mathrm{dl}$ of urine or $\geq 2++$ of proteinuria on urine dipstick measurement) and or abnormal biochemical and hematological findings $\mathrm{s}^{1-2}$.

Although all forms of HDP are potentially life-threatening, pre-eclampsia is the severest causing more than 500,000 and 70,000 global neonatal and maternal deaths respectively ${ }^{2,4}$. During pregnancy, delivery and the immediate postpartum periods, pre-eclampsia can be complicated by convulsions in the mother (Eclampsia), stroke, antepartum hemorrhage ( for example abruptio placentae as a consequence of associated hypertension in pre-eclampsia), postpartum hemorrhage (for example from Disseminated Intra-vascular Coagulopathy -DIC), acute kidney failure, pulmonary edema, liver rupture and hemolysis, elevated liver enzymes and low platelets (HELLP's syndrome) ) $^{3-5}$. All these contribute to the impact of HDP on maternal and perinatal survival and wellbeing.

Besides HDP's contribution to immediate pregnancy-related complications, HDP is also associated with long-term maternal health outcomes. Increasing bodies of knowledge link 
prior history of HDP with long-term asymptomatic and symptomatic cardiovascular disease risk $^{6-9}$. Studies have shown 3.5 folds increase in risk of asymptomatic heart disease four to ten years following $\mathrm{HDP}^{9}$ and three-seven folds increase risk of future cardiovascular diseases and $40 \%$ risk of structural and functional cardiac anomalies up to 1 year after delivery ${ }^{10}$. Women with previous HDP-complicated pregnancies (especially those with history of pre-eclampsia) are prone to developing insulin resistance and its associated metabolic problems of dyslipidemia and hyperinsulinemia generally referred to as metabolic syndrome, within few months to several years ${ }^{11-16}$.

Evidence also suggests that HDP (mainly pre-eclampsia) affects renal function and morphology, leads to persistent microalbuminuria after delivery, and could lead to chronic and end-stage renal diseases (both pre-eclampsia and gestational hypertension) ${ }^{17-20}$. Another chronic medical condition associated with history of HDP is type II diabetes mellitus, with evidence emerging from different populations. Some authors have suggested that preeclampsia confers similar risk to developing type II diabetes mellitus as do obesity and smoking ${ }^{21-23}$. Two to four folds increased risk of developing type II diabetes mellitus in HDPaffected pregnancies, compared to normotensive, have been reported in some series ${ }^{24-26}$.

Other complications that could occur during postpartum period in HDPs in the absence of proper management includes thromboembolism and unresolved hypertension leading to chronic hypertension in some women ${ }^{27}$. The foregoing suggests the importance of HDP to health of women both during their reproductive years and beyond.

\section{Global burden of hypertensive disorders in pregnancy}

Hypertension impacts about one billion adults worldwide, with 9 million related annual death $s^{28}$. HDP is a global health problem complicating between $5-10 \%$ of pregnancies and causing more than 500,000 and 70,000 global neonatal and maternal deaths respectively $1,2,28,29$. Because of rising prevalence of obesity and maternal age due to delays in starting a family, the prevalence and burden of HDP will likely increase over the next few decades ${ }^{30}$. As a consequence of the recent surge in hypertension in Africa, HDP are among the top causes of maternal and perinatal mortality in this continent ${ }^{31}$. As much as $10 \%$ of African women 
would develop any of HDP types during pregnancy or postpartum period, with prevalence significantly higher in sub-Saharan than in north Africa ${ }^{28}$.

\section{Burden of HDP in Nigeria}

HDP, as in the rest of the world, affects $5-10 \%$ of pregnancies in Nigeria. With the projected 7.4 million births in Nigeria by the year 2020 (37.269 births per 1000 population) ${ }^{32}$, HDP can be estimated to affect between $350,000-700,000$ pregnancies in 2020 alone. While HDP is only second to postpartum hemorrhage as cause of deaths in Nigeria in general, it is the leading cause of maternal mortality and morbidity in tertiary referral centers accounting for $23 \%$ of maternal deaths in this setting ${ }^{33}$. With the growing evidence linking HDP to future risks of chronic cardiovascular and metabolic disorders as identified in earlier sections ${ }^{6-20}$, HDP would constitute triple burden to health of women and children in Africa in general and Nigeria in particular. This burden will include effects on health of women during pregnancy, childbirth and postpartum period ${ }^{3-5}$, perinatal complication due to prematurity or intrauterine growth restriction ${ }^{2,4}$ and chronic health outcomes after reproductive life years ${ }^{6-}$ 20.

\section{Thesis objective}

The objective of this thesis is to explore management practices of HDP during antenatal and postnatal periods and to assess short- and intermediate-term health outcomes for women following HDP-complicated pregnancies in Nigeria. The thesis will examine:

1. Quality of care (including facilitating and militating factors) for women with HDP in Nigeria

2. Medical health outcome for women within one year of delivery of HDP-complicated pregnancies

3. Community-based approach to understanding and managing HDP

This thesis, therefore, holistically address pertinent issues around care for women with HDP right from their communities and their interaction with the primary, secondary and tertiary health systems. Because this research was conducted on the platform of non-governmental 
organizations' (NGOs') service delivery setting in a low-resource country, it discusses the suitability of NGO's derived data to contribute to body of knowledge in the management of HDP in low-resource setting and elsewhere.

\section{Thesis outline}

Part I of this thesis will look at quality of care issues for women with hypertensive disorders in pregnancy in Nigeria during antenatal and postnatal periods (chapter $2 \& 3$ ). In part II, the thesis will assess prevalence and incidence of three medical conditions known to be associated with prior hypertensive disorders in pregnancy namely persistent (chronic) hypertension, metabolic syndrome and chronic kidney diseases -CKD)- (chapter 4-6). Finally, Part III will review HDP through the lens of the survivors (chapter 7) and will suggest an integrated community care model for women with HDP (chapter 8). 


\section{References}

1. Laura AM, Peter VD, William S; Mathew M (2016). The FIGO textbook of pregnancy hypertension: An Evidence-based guide to monitoring, prevention and management. ISBN: 978-0-9927545-5-6

2. Mark AB, Laura AM, Louise CK et al. Hypertensive Disorders of Pregnancy ISSHP Classification, Diagnosis, and Management Recommendations for International Practice. DOI: 10.1161/HYPERTENSIONAHA.117.10803

3. Nankali A, Malek-khosravi SH, Zangeneh M, Rezaei M, Hemati Z, Kohzadi M. Maternal complications associated with severe pre-eclampsia. J Obstet Gynaecol India. 2013 Apr; 63(2): 112-115. doi: $10.1007 / \mathrm{s} 13224-012-0283-0$

4. Alicia ML. Effects of preeclampsia on the mother, fetus and child. Pregnancy and Birth, Fetal Monitoring. Retrieved June 112020 from https://www.obgyn.net/pregnancy-andbirth/effects-preeclampsia-mother-fetus-and-child

5. Cheryl B, Monique C. Preeclampsia and Stroke: Risks during and after Pregnancy. Stroke Research and Treatment 2011. https://doi.org/10.4061/2011/858134

6. Theilen LH, Fraser A, Hollingshaus MS et al. All-cause and cause-specific mortality after hypertensive disease of pregnancy. Aust N Z J Obstet Gynecol. 2016; 128:238-244. doi: 10.1097/AOG.0000000000001534

7. Theilen L, Meeks H, Fraser A, Esplin MS, Smith KR, Varner M. Long-term mortality risk and life expectancy following recurrent hypertensive disease of pregnancy. Am J Obstet Gynecol. 2017;216(suppl 1): S32-S33

8. Tooher J, Thornton C, Makris A et al. Hypertension in pregnancy and long-term cardiovascular mortality: a retrospective cohort study. Am J Obstet Gynecol. 2016; 214:722. e1-722. e6. doi: 10.1016/j.ajog.2015.12.047.

9. Ghossein-Doha C, Van neer J, Wissink B et al. Pre-eclampsia: an important risk factor for asymptomatic heart failure. Ultrasound Obstet Gynecol 2017; 49: 143-149.

10. Bellamy L, Casas JP, Hingorani AD, Williams DJ. Pre-eclampsia and risk of cardiovascular disease and cancer in later life: systematic review and meta-analysis. British Medical Journal. 2007; 335:974. doi: 10.1136/bmj.39335. 385301. 
11. Joe“| G, Yves G, Jean-Marie M, Jean-Claude F. Previous Hypertensive Disease of Pregnancy Is Associated with Alterations of Markers of insulin resistance. Hypertension. 2007; 49:10561062.

12. Kaaja R, Laivuori $H$, Laakso M, Tikkanen MJ, Ylikorkala O. Evidence of a state of increased insulin resistance in preeclampsia. Metabolism. 1999; 48:892-896.

13. Solomon CG, Carroll JS, Okamura K, Graves SW, Seely EW. Higher cholesterol and insulin levels in pregnancy are associated with increased risk for pregnancy-induced hypertension. Am J Hypertens. 1999;12: 276-282.

14. Laivuori H, Tikkanen MJ, Ylikorkala O. Hyperinsulinemia 17 years after preeclamptic first pregnancy. J Clin Endocrinol Metab. 1996;81: 2908-2911.

15. Sattar N, Ramsay J, Crawford L, Cheyne H, Greer IA. Classic and novel risk factor parameters in women with a history of preeclampsia. Hypertension. 2003; 42:39-42.

16. Nisell H, Erikssen C, Persson B, Carlstrom K. Is carbohydrate metabolism altered among women who have undergone a preeclamptic pregnancy? Gynecol Obstet Invest. 1999; 48:241-246

17. Gaber LW, Spargo BH, Lindheimer MD. Renal pathology in pre-eclampsia. Baillieres Clin Obstet Gynaecol 1994; 8:443-68

18. Bar J, Kaplan B, Wittenberg C, et al. Microalbuminuria after pregnancy complicated by preeclampsia. Nephrol Dial Transplant 1999; 14:1129-32.

19. McDonald SD, Han Z, Walsh MW, et al. Kidney disease after preeclampsia: a systematic review and meta-analysis. Am J Kidney Dis 2010; 55:1026-39

20. I-Kuan W, Chih-Hsin M, Yi-Chih C et al. Association between hypertensive disorders during pregnancy and end-stage renal disease: a population-based study. Canadian Medical Association Journal, February 19, 2013, 185(3)

21. Callaway LK, Lawlor DA, O'Callaghan $M$ et al. Diabetes mellitus in the 21 years after a pregnancy that was complicated by hypertension: findings from a prospective cohort study. Am J Obstet Gynecol 2007; 197:492. e1-7 
22. Engeland A, Bjorge T, Kjersti DAK et al. Risk of diabetes after gestational diabetes and preeclampsia. A registry-based study of 230,000 women in Norway. Eur J Epidemiol 2011; 26:157-63

23. Wang IK, Tsai IJ, Chen PC et al. Hypertensive disorders in pregnancy and subsequent diabetes mellitus: a retrospective cohort study. Am J Med 2012; 125:251-7.

24. Lykke JA, Langhoff-Roos J, Sibai BM et al. Hypertensive pregnancy disorders and subsequent cardiovascular morbidity and type 2 diabetes mellitus in the mother. Hypertension 2009; 53:944-51.

25. Feig DS, Zinman B, Wang $X$ et al. Risk of development of diabetes mellitus after diagnosis of gestational diabetes. Canadian Medical Association Journal 2008; 179:229-34.

26. Agrawal S, Fledderjohann J. Hypertensive disorders of pregnancy and risk of diabetes in Indian women: a cross-sectional study. British Medical Journal Open 2016;6: e011000. doi:10.1136/bmjopen-2015011000

27. Royal College of Obstetricians and Gynecologists. Hypertension in pregnancy: The management of hypertensive disorders during pregnancy. NICE Clinical guideline; August 2010. Revised reprint January 2011

28. Jean JN, Jean JB, Ulrich FN, Ahmadou MJ, Arnaud DK, Jobert RN, Joel FD. The burden of hypertensive disorders in Africa: A systematic review and meta-analysis. J Clin Hypertens. 2019; 21:479-488.

29. Foo L, Tay J, Lees CC, McEniery CM, Wilkinson IB. Hypertension in Pregnancy: natural history and treatment options. Curr Hypertens Rep (2015) 17: 36; DOI 10.1007/s11906-015-0545-1

30. Umesawa M, Kobashi G. Epidemiology of hypertensive disorders in pregnancy: prevalence, risk fac tors, predictors and prognosis. Hypertens Res. 2017;40(3):213-220.

31. Say L, Chou D, Gemmill A et al. Global causes of maternal death: a WHO systematic analysis. Lancet Glob Health. 2014;2(6): e323-e333.

32. Macrotrends. Nigeria birth rate 1950 - 2020. Retrieved July 20, 2020 from https://www.macrotrends.net/countries/NGA/nigeria/birth-rate

33. Oladapo OT, Adetoro OO, Ekele BA et al. Nigeria Near-miss and Maternal Death Surveillance Network. When getting there is not enough: a nationwide cross-sectional study of 998 
maternal deaths and 1451 near-misses in public tertiary hospitals in a low-income country. $\mathrm{Br}$ J Obstet Gynaecol 2015; DOI: 10.1111/1471-0528.13450. 




\section{Part 1}

Quality of care for women with hypertensive disorders in pregnancy 


\section{Chapter 2}

Detecting and managing hypertensive disorders in pregnancy: a cross-sectional analysis of the quality of antenatal care in Nigeria

Angela Salomon

Salisu Ishaku

Karen R. Kirk

Charlotte E. Warren

BMC Health Services Research (2019) 19:411. https://doi.org/10.1186/s12913-019-4217-8 


\section{Abstract}

Background: Nigeria has one of the highest rates of maternal mortality in the world (576/100,000 births), with a significant proportion of death attributed to hypertensive disorders in pregnancy (HDP). High quality antenatal care (ANC) plays a crucial role in early detection and management of HDP. We conducted an assessment of quality of antenatal care, and its capacity to detect and manage HDP, in two tiers of Nigerian facilities, with the aim of describing the state of service delivery and identifying the most urgent gaps.

Methods: Quality of antenatal care was assessed and compared between primary healthcare centers (PHCs) ( $n=56$ ) and hospitals (secondary + tertiary facilities, $n=39$ ) in seven states of Nigeria. A cross-sectional design captured quality of care using facility inventory checklists, semi-structured interviews with healthcare providers and clients, and observations of ANC consultations. A quality of care framework and scoring system was established based on aspects of structure, process, and outcome. Average scores were compared using independent sample t-tests and measures of effect were assessed by multivariable linear regression.

Results: All domains of quality except provider interpersonal skills scored below $55 \%$. The lowest overall scores were observed in provider knowledge $(49.9 \%)$ and provider technical skill (47.7\%). PHCs performed significantly worse than hospitals in all elements of quality except for provider interpersonal skills. Provider knowledge was significantly associated with their level of designation (i.e., obstetrician vs. other providers).

Conclusions: In order to provide high quality care, ANC in Nigeria must experience massive improvements to inventory, infrastructure and provider knowledge and training. In particular, ANC programs in PHCs must be revitalized to minimize the disparity in quality of care provided between PHCs and hospitals. The relatively low quality of care observed may be contributing to Nigeria's high rate of maternal mortality and burden of disease attributed to HDP.

Keywords: Antenatal care - maternal health - quality of care - health systems, Hypertensive disorders in pregnancy - primary health care 


\section{Background}

Antenatal care (ANC) describes the services offered to pregnant women, including health promotion and communication, screening and diagnosis, disease prevention, and emotional and psychological support. Quality, evidence-based ANC plays a crucial role in improving the lives of pregnant women, and in setting the groundwork for healthy motherhood and infant development [1]. As of 2015, hypertensive disorders in pregnancy (HDP), including preeclampsia and eclampsia, were the greatest cause of facility-based maternal mortality (29.0\%) in Nigeria [2]. Broadly speaking, HDP are characterized by elevated blood pressure, proteinuria, and many hematologic, hepatic, neurologic and renal changes that can result in adverse maternal and neonatal outcomes including intrauterine growth restriction, oligohydramnios (low amniotic fluid), placental abruption, and fetal death [3]. Because HDP tend to have easily detectable clinical parameters [4], increasing utilization of quality ANC is essential to reducing maternal mortality caused by this class of disease. Globally, an estimated $81 \%$ of pregnant women attend at least one antenatal care visit, while only $56 \%$ attend at least four [5]. In regions of sub-Saharan Africa, this number is further reduced [6], and in Nigeria specifically, only half of pregnant women attend at least four visits, ranging from $30 \%$ in north-western states to $87 \%$ in the southwest $[7,8]$. While increasing access to ANC services is important, it is also critical that the services received are of good quality. Quality of Care ( $\mathrm{QOC})$, as described by Donabedian [9], depends on three components: structure (adequacy of physical environment and systems), process (components of care delivered), and outcomes (satisfaction/status of clients) $[9,10]$. That is to say, facilities should be well stocked with essential commodities, services should be provided by competent healthcare workers, and clients should leave well-informed, satisfied, and respected. In Nigeria, primary healthcare centers (PHCs) are the first points of contact for many pregnant women, especially for the majority of rural dwellers; however, service readiness in PHCs is often sub-standard compared to hospital counterparts $[11,12]$. Recently, a landscape analysis of pre-eclampsia and eclampsia in Nigeria [13] showed limited capacity at PHCs for providing even the most basic emergency obstetric and neonatal care. Previous research has explored factors affecting 
quality of ANC in Nigeria but has been restricted to private health facilities and small geographic areas and has used a limited scope of quality of care [14-16]. This paper explores the quality of ANC in seven states across the six geopolitical zones of Nigeria using a wellestablished framework for assessing QOC, with a focus on its capacity to detect and manage hypertensive disorders in pregnancy. Furthermore, it examines disparities in the quality of ANC provided between PHCs and hospitals.

\section{Methods}

\section{Study participants}

Following discussion with the Nigerian Federal Ministry of Health and the donor, seven states covering the country's six geo-political zones were chosen as study sites - Sokoto, Bauchi, Katsina, Kogi, Cross River, Ebonyi, and Ondo (Fig. 1). In consultation with the states' Ministries of Health, ninety-five public facilities with ANC units were purposively selected from within these states to represent diversity in facility type $(\mathrm{PHCs}=56$, secondary/tertiary hospitals $=$ 39). Following an inventory assessment at 95 selected facilities, researchers conducted service provider interviews $(n=200)$ from a subset of 93 facilities; 2 facilities were excluded as no willing providers were present on the day of the inventory assessment. Researchers also observed 135 ANC consultations and conducted 135 client exit interviews from a subset of 26 facilities. The subset of facilities used for consultation observations and client interviews was chosen using convenience sampling, based on the location of the facility (considering difficult terrains), as well as the functionality of the facility (facility operating hours), while ensuring a representative sample across facility level and region.

\section{Quality of Care framework}

The framework used for measuring quality of ANC was adapted from Warren et al. [17] and included many of the essential elements of antenatal care as informed by WHO guidelines [1]. It was further tailored to include indicators specifically pertaining to the capacity of the ANC to detect and manage HDPs. The framework incorporated three elements of QOC as described by Donabedian [9], Bruce [18], and Hulton [19]: structure, process, and outcome. 
Structure encompassed factors related to inventory and infrastructure (facility guidelines, equipment, supplies, drugs, referral mechanisms, etc.), as well as human resources (provider knowledge and training). Process encompassed the range of services provided, information and documentation shared with the client, the practitioner's competency in history taking and physical examinations, and their interpersonal skills. Outcome encompassed client experiences, satisfaction, and health comprehension. Table 1 shows the indicators used to create structure, process, and outcome scores. Scores were calculated as the average cumulative number of points received by each unit of observation (facility, provider, ANC consultation, or client interview). All scores are reported as a percent of the total possible score.

\section{Data collection}

This paper uses cross sectional data collected during a landscape analysis that assessed the health facility and provider capacity to prevent, detect, and manage pre-eclampsia and eclampsia in seven states in Nigeria.

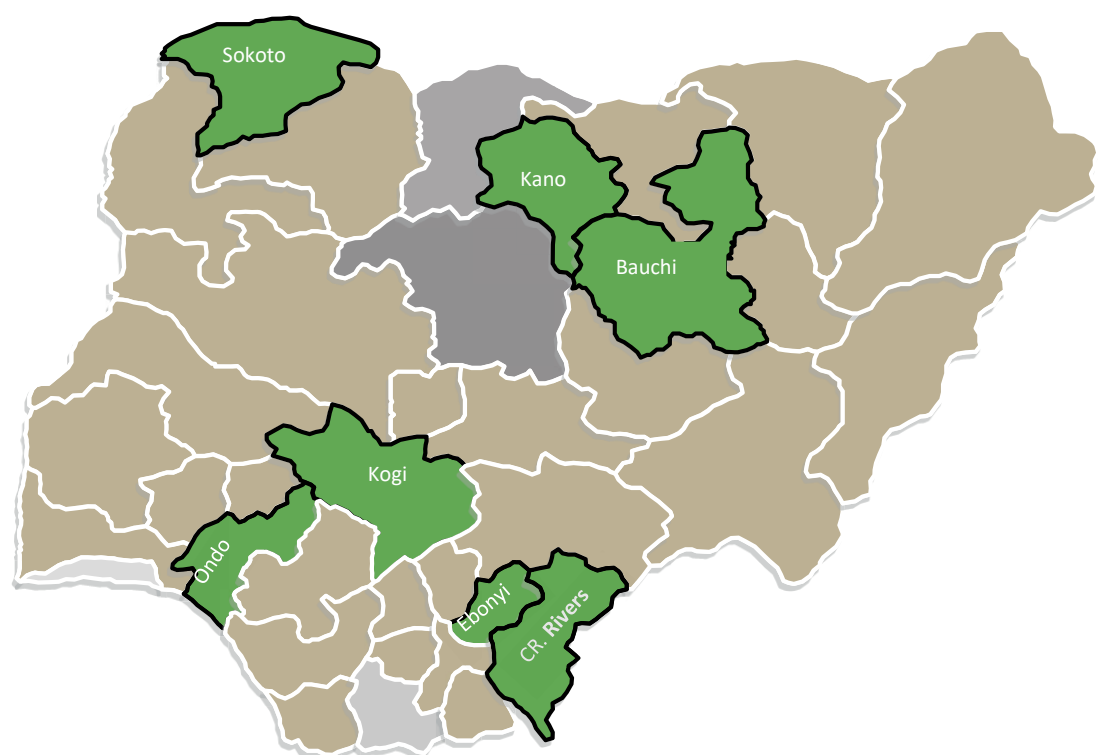

Fig. 1. Map of study sites. Data was collected from 95 participating hospitals in 7 states across the 6 geopolitical zones of Nigeria. Geopolitical zones are divided based on similarity in culture, ethnic group, and common history. Including all hospitals from all geopolitical zones helps to ensure representative national results. Rights to this image are owned by the author 
Data collection occurred over a three-month period, from June-August 2015.

\section{Structure}

Inventory and infrastructure $(n=95)$ were assessed with a facility inventory checklist for a maximum score of 38 points. Provider knowledge, competence, and training was assessed using semi-structured interviews with the ANC service providers $(n=200)$ for a maximum score of 66 points (Table 1).

Process

Client-provider interactions $(n=135)$ were observed and documented using a standardized checklist to assess the provider's technical skills for a maximum score out of 37 , and the provider's interpersonal skills for a maximum score out of 11 (Table 1).

\section{Outcome}

Following their ANC visit, researchers conducted semi-structured exit interviews with clients $(n=135)$ to assess their perception, satisfaction, and knowledge of services received, for a maximum score out of 16 (Table 1 ).

\section{Data analysis}

We compared mean scores obtained from PHCs and hospitals in each QOC category using one-tailed independent sample t-tests ( $\mathrm{HO}$ : secondary facility = PHCs, HA: secondary facility > PHC); variances were assumed unequal if Levene's Test for Equality was significant. For all categorical variables, significant differences between facility type were determined using the Pearson chi-square test; Fisher's Exact test was used in cases where individual cell counts were below 5 .

We then conducted two multivariable linear regression models to identify independent variables associated with provider knowledge and outcome scores. Variables in model 1 included facility level, provider age, gender, and designation; variables in model 2 included facility level, client age, education, and socio-economic status (SES). Clustering within facility was adjusted for using a robust estimate of variance (sandwich estimator). We developed a single variable for SES through principal component analysis of indicators pertaining to wealth, household facilities, and assets (including vehicles, land, and livestock owned). We classified SES as either low, medium or high based on whether the individual fell into the bottom, middle, or top tertile [20]. A significance threshold of $p=0.05$ was used for all 
analyses. Individual observations with $>50 \%$ of data missing were excluded from analysis. Where appropriate, means (with standard deviations), coefficients (with 95\% confidence intervals), and p-values are reported. Data were stored, cleaned, and analyzed using STATA v $15.1[21]$

\section{Result}

Figure 2 shows an overview of the scores received in each of the five categories by all facilities, PHCs, and Hospitals.

\section{Descriptive characteristics}

Client and provider characteristics are described in Table 2. The average client was 27.78 years of age, with those visit- ing PHCs being significantly younger than those visiting hospitals (24.9 vs. 28.3 years; $p=0.001)$. Most women had completed at least secondary level of schooling $(30.1 \%)$, and the level of education attained was significantly associated with facility type, with a much higher percentage of women at hospitals having attended postsecondary school $(p<0.001)$. Those identifying as Muslim were approximately three times more likely to attend a PHC than a hospital facility $(p=0.001)$, likely because the majority of PHCs sampled were in Northern states, which are pre- dominantly Muslim. There were no significant differences between PHCs and hospitals in the client's SES, marital status, or spending decision habits. The mean age of providers (39.1 years) was similar at both PHCs and hospitals, and the majority were female (81.6\%). In PHCs, most providers were Community Health Extension Workers (CHEWs) (64.8\%), followed by nurse/midwives (18.1\%); this was significantly different from hospitals, where the majority of provides were nurses/midwives $(66.7 \%)$, followed by general practitioners $(15.6 \%)(p<0.001)$.

\section{Structural attributes of care: inventory/infrastructure}

Table 3 shows the structural attributes related to facility inventory and infrastructure. Out of a total of 38 points, PHCs scored significantly lower than hospitals $(47.5 \%$ vs. $60.0 \%, p<$ 0.001). PHCs also scored significantly lower in domains of general infrastructure $(40.8 \% \mathrm{vs}$. 
$54.3 \% ; p=0.0116)$, general equipment (54.2\% vs. $64.6 \%, p=0.0304)$, referral mechanisms (54.9\% vs. $67.3 \% ; p=0.0161)$ and capacity for HDP specific services (38.8\% vs. $68.6 \% ; p<$ 0.0001). There were no significant differences between facility type in scores for data collection tools (90.5\%), HDP guidelines (33.9\%), or availability of drugs associated with HDPs (38.5\%).

Structural attributes of care: Provider knowledge/training

Table 3 shows the structural attributes related to provider knowledge and training. Out of a total of 66 points, providers in PHCs scored significantly lower than those in hospitals $(46.0 \%$ vs. $54.1 \%, p=0.002$ ). 


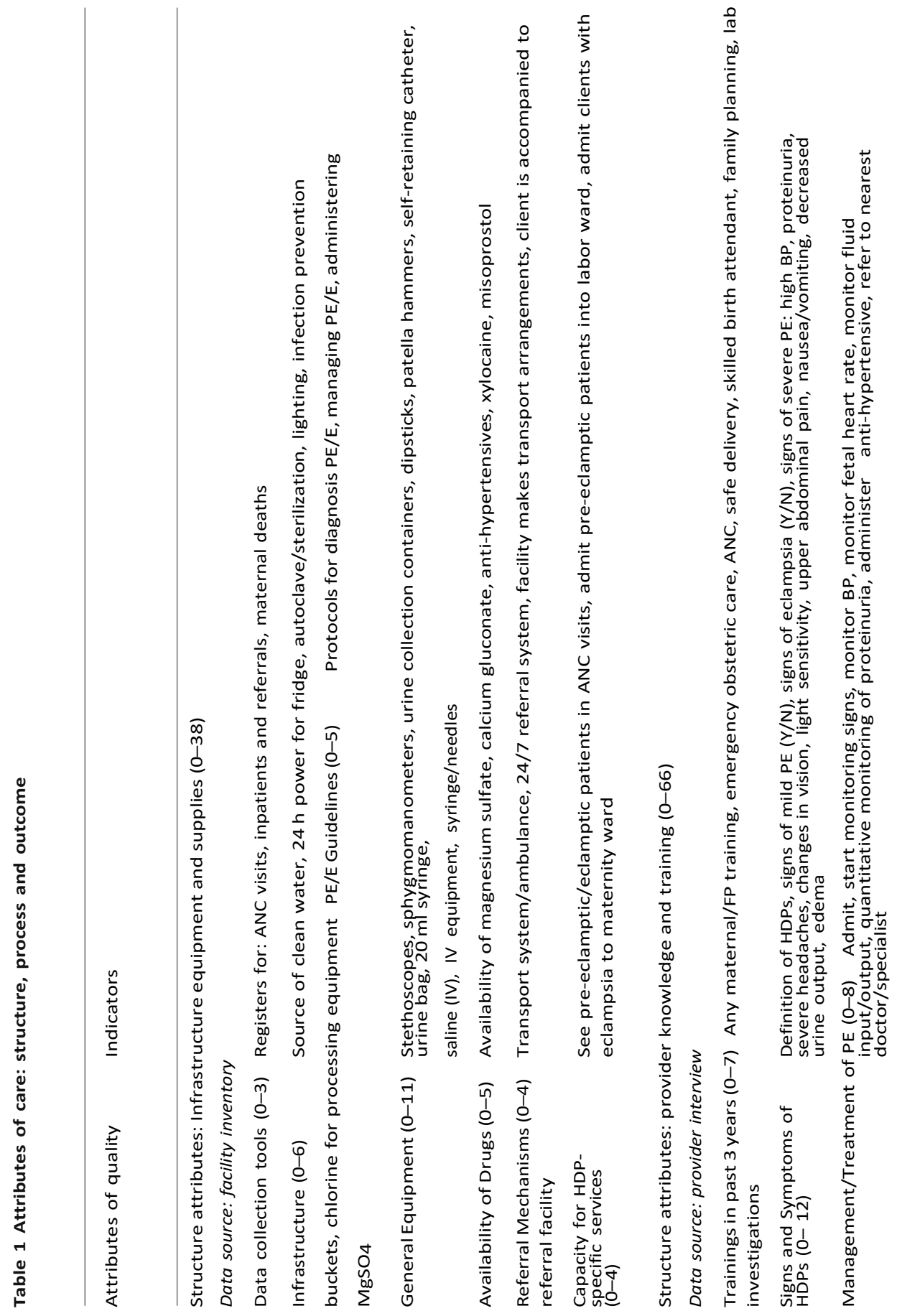




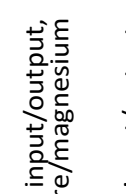

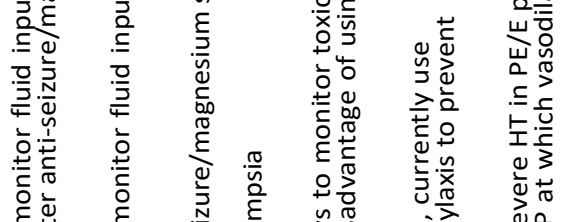

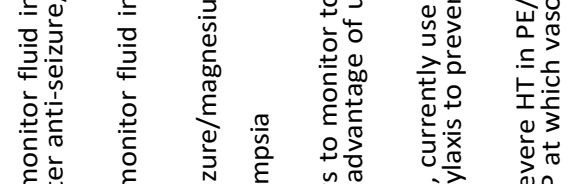

है

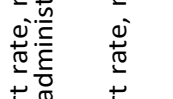

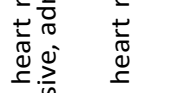

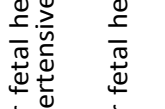

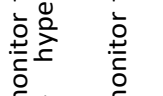

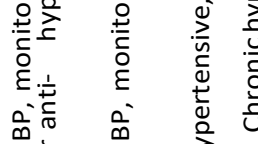

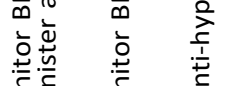

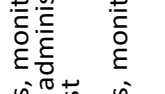

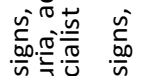

:

政产

है응

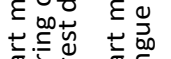

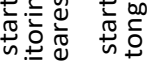

हैं

एक्ष

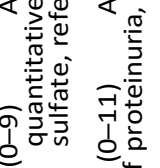

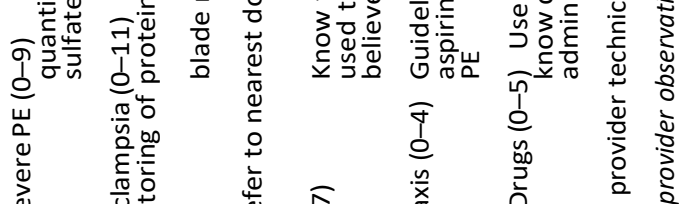

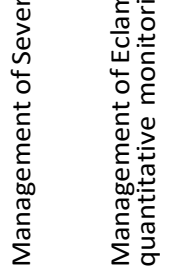

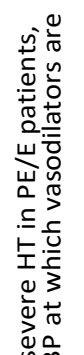

온

ㅎํㅇㅇㅛ

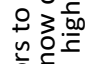

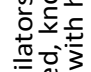

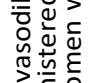

这高交

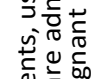

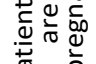

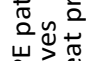

山्य.

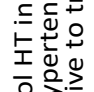

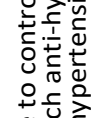

:

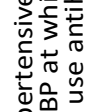

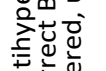

先

ऽ) $3 . \frac{5}{5}$

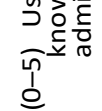

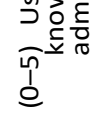

品

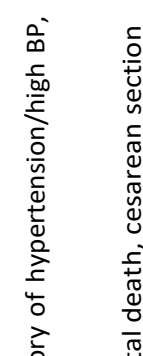

总

है

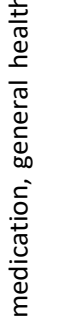

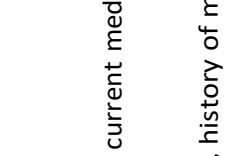

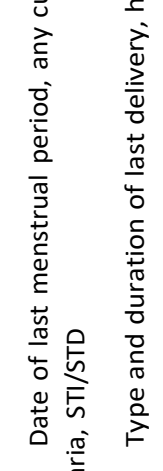

更

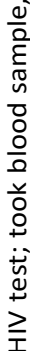

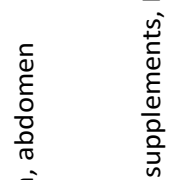

$\frac{0}{8} \quad \frac{n}{0}$

竞

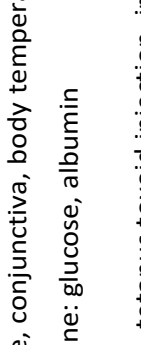

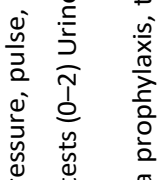

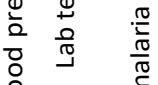

잉

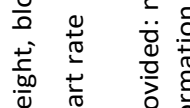

3

a

कิ

की

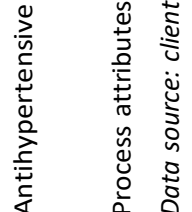

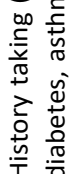

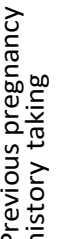

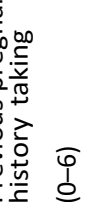

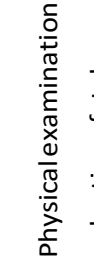

㟧

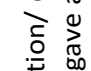

نे

흘 을

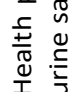




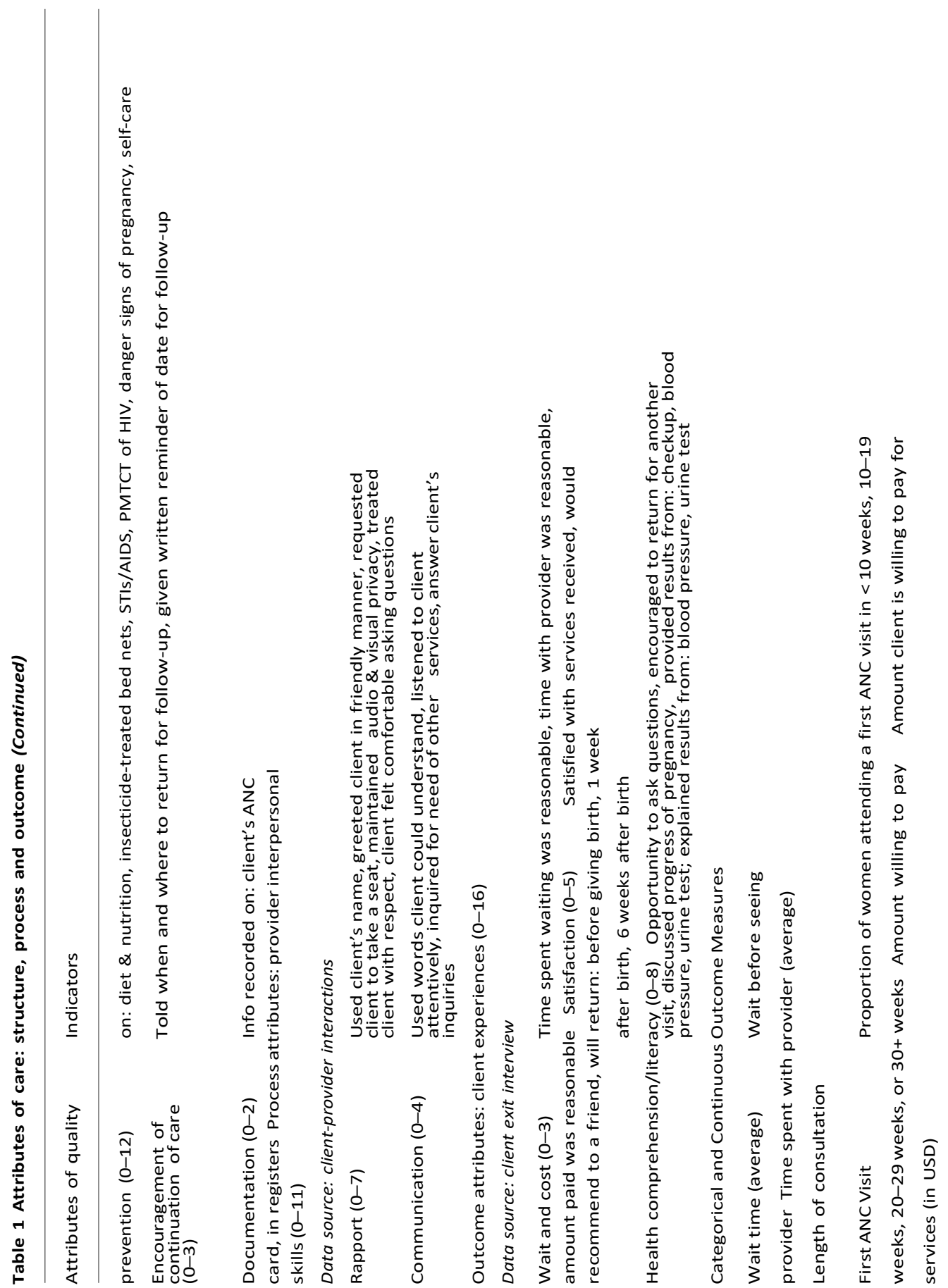




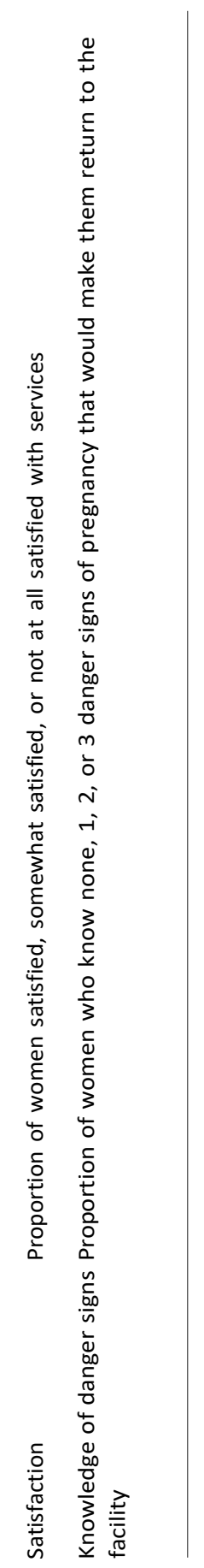


PHCs scored lower than hospitals in additional facets of provider knowledge including signs and symptoms of HDPs (60.1\% vs. $67.8 \% ; p=0.019)$, how to manage severe pre- eclampsia $(49.2 \%$ vs. $59.0 \% ; p=0.032)$ and eclampsia $(49.7 \%$ vs. $65.2 \%)$, correctly diagnosing HDPs $(58.0 \%$ vs. $73.6 \% ; p=0.000)$, correct use of magnesium sulfate $(15.9 \%$ vs. $31.1 \%, p=0.000)$, and use of antihypertensive drugs to manage mild $\operatorname{HDP}(13.6 \%$ vs. $23.5 \%, p=0.001)$. The difference observed in overall provider knowledge between PHCs and hospital facilities was no longer significant after regression adjustment for provider demographic variables including age, gender, type of provider, and length of time working at facility (Fig. 3). However, overall provider knowledge was significantly associated with provider type; using maternal health specialists as the reference (obstetricians/gynecologists), it was found that general practitioners, nurses/midwives, CHEWs, and CHOs scored lower by 12.0 points $(p=0.001), 15.4$ points $(p=$ $0.000), 23.8$ points $(p=0.000)$ and 18.5 points $(p=0.000)$, respectively. Further adjustment for clustering by specific facility did not quantitatively or qualitatively change the results of the regression analysis.

\section{Process attributes of care: provider technical skills}

Table 4 shows the process attributes related to provider technical skills. Out of a total of 37 points, PHCs scored significantly lower than hospitals ( $42.2 \%$ vs. $49.8 \%, p=0.0017)$. There were also significant differences between facility type for skills in general history taking $(42.2 \%$ vs. $61.0 \% ; p<0.001)$ and history taking for women with a previous pregnancy $(39.6 \%$ vs. $58.8 \% ; p=$ 0.0073). There were no significant differences in scores for physical examinations, lab tests, documentation, health promotion/prevention of disease, or encouragement of follow-up, but all categories except for documentation and encouragement of follow-up scored below $70 \%$ of potential points.

\section{Process attributes of care: provider interpersonal skills}

Table 4 also shows the process attributes related to provider interpersonal skills. Out of a total score of 11 , PHCs and hospitals scored highly -85.3 and $84.9 \%$, respectively. There were no significant differences observed in the scores of interpersonal sub- categories, or in the cumulative interpersonal score between PHC and hospital care providers. However, individual 
indicators within the rapport-building and communications sub-categories were found to significantly differ (see Additional file 1). For example, PHCs more often used the client's name (97.5\% vs. $89.5 \% ; p=0.016)$ and greeted in a friendly manner ( $100 \%$ vs. $85.4 \% ; p=0.011)$, but were less likely to maintain audio privacy $(67.5 \%$ vs. $92.6 \% ; p<0.001)$ and visual privacy $(65.0 \%$ vs. $90.5 \%$; $p$ <.001), or inquire for need of other services (35.9\% vs. $83.3 \%$; $p=0.022$ ). 


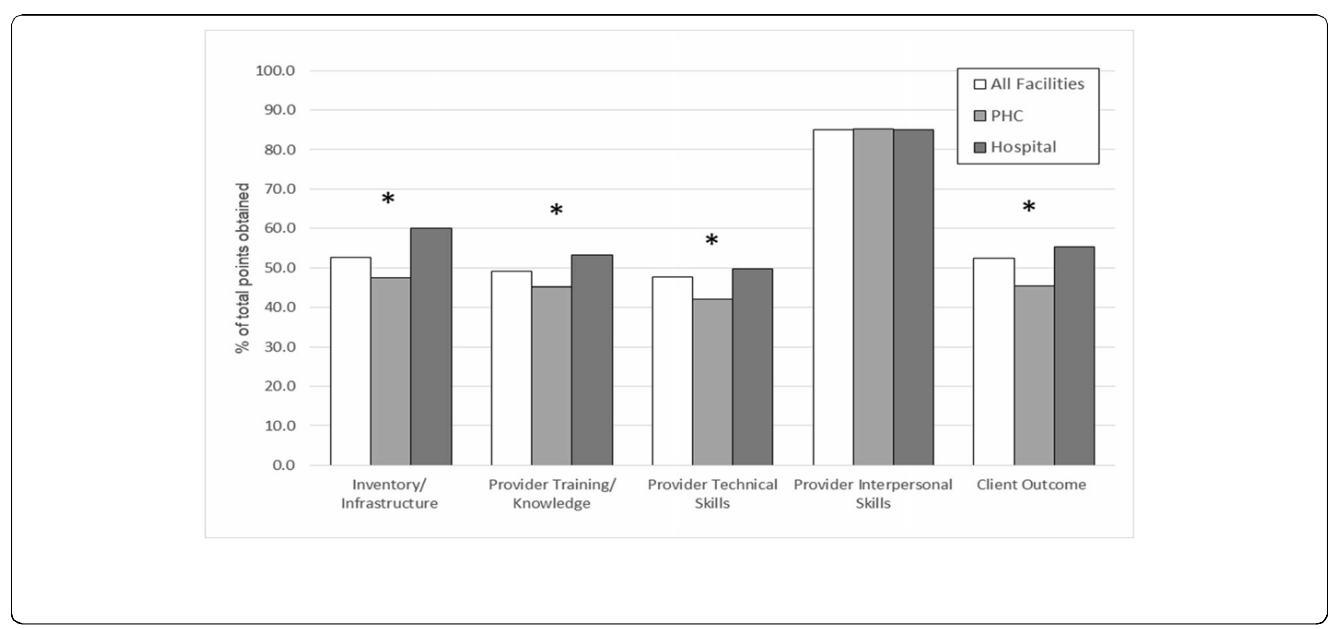

Fig. 2 Comparison of domains of quality of care across facility type. All domains except for interpersonal skills obtained a cumulative score of below $55 \%$. The lowest overall scores were obtained in provider and provider skill (47\%) and provider training/knowledge (49\%), while the highest scores were obtained in provider interpersonal skills (85\%). PHCs scored lower than hospitals in all domains except provider interpersonal skills

\section{Determinants of Provider Knowledge}

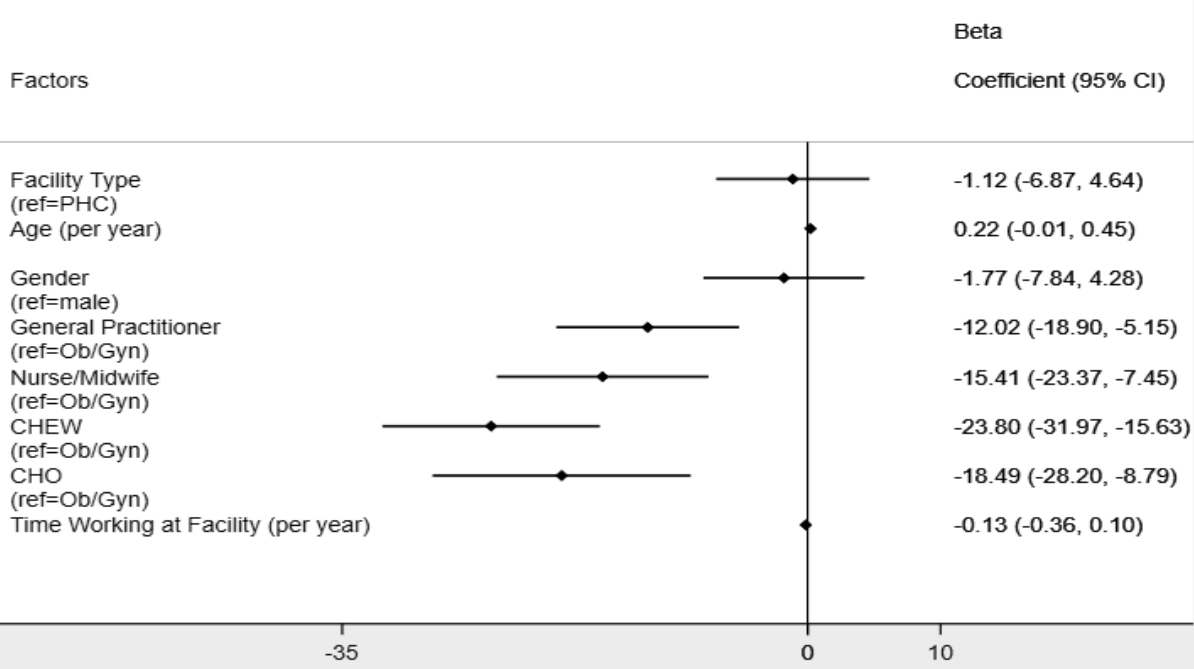

Fig. 3 Linear regression coefficients examining provider knowledge score. Each row represents a variable included in the multivariable linear regression assessing factors associated provider knowledge scores. After adjustment for provider sociodemographic factors, the level of facility (primary vs. secondary) was no longer significant. Compared to obstetricians/gynecologists (reference group), each of the other provider types (general practitioner, nurse/midwife, $\mathrm{CHEW}$, and $\mathrm{CHO}$ ) obtained significantly lower knowledge scores 


\begin{tabular}{|c|c|c|c|c|}
\hline Client characteristics & $\begin{array}{l}\text { Total } \\
(n=135)\end{array}$ & $\begin{array}{l}\text { PHC } \\
(n=38)\end{array}$ & $\begin{array}{l}\text { Hospital } \\
(n=97)\end{array}$ & $P$-Value \\
\hline Mean age (years) $\pm S D$ & $27.87 \pm 8.23$ & $24.89 \pm 5.88$ & $28.32 \pm 5.15$ & 0.001 \\
\hline Mean gravidity $\pm S D$ & $2.79 \pm 2.05$ & $3.16 \pm 2.49$ & $2.65 \pm 1.84$ & 0.256 \\
\hline Mean gestational age (weeks) \pm SD & $28.26 \pm 8.43$ & $30.13 \pm 8.56$ & $27.43 \pm 8.29$ & 0.120 \\
\hline Mean gestational age at 1st ANC visit (weeks) & $20.83 \pm 7.78$ & $21.9 \pm 8.06$ & $20.49 \pm 7.71$ & 0.413 \\
\hline \multicolumn{5}{|l|}{ \pm SD Mean \# ANC visits ${ }^{b}$} \\
\hline Gestational age $<20$ weeks $(n=13)$ & $2.23 \pm 1.79$ & $2.00 \pm 2.00$ & $2.33 \pm 1.80$ & 0.771 \\
\hline Gestational Age $20-29$ weeks $(n=27)$ & $2.15 \pm 1.54$ & $2.60 \pm 1.52$ & $2.05 \pm 1.56$ & 0.477 \\
\hline Gestational age $30+$ weeks $(n=64)$ & $3.43 \pm 2.09$ & $3.39 \pm 1.9$ & $3.45 \pm 2.21$ & 0.920 \\
\hline Education [n (\%)] & & & & $<0.001$ \\
\hline Primary or below primary & $41(30.4)$ & $18(47.4)$ & $23(23.7)$ & \\
\hline Secondary & $41(30.1)$ & $19(50)$ & $22(22.7)$ & \\
\hline Postsecondary & $53(39.3)$ & $1(2.6)$ & $52(53.6)$ & \\
\hline Religion [n (\%)] & & & & 0.001 \\
\hline Christian & $60(44.4)$ & $8(21.1)$ & $52(53.6)$ & \\
\hline Muslim & $75(55.6)$ & $30(78.9)$ & $45(46.4)$ & \\
\hline Marital status [n (\%)] & & & & $1.000^{\mathrm{a}}$ \\
\hline Married/living together & $130(96.3)$ & $37(97.4)$ & $93(95.9)$ & \\
\hline Divorced/separated/widowe & $5(3.7)$ & $1(2.6)$ & $4(4.1)$ & \\
\hline d Health insurance coverage & & & & 0.191 \\
\hline Yes & $19(23.8)$ & $2(11.8)$ & $17(27.0)$ & \\
\hline No & $61(76.3)$ & $15(88.2)$ & $46(73.0)$ & \\
\hline Socioeconomic status [n (\%)] & & & & 0.051 \\
\hline Low & $50(37.0)$ & $9(23.7)$ & $41(42.3)$ & \\
\hline Medium & $63(46.7)$ & $24(63.2)$ & $39(40.2)$ & \\
\hline High & $22(16.3)$ & $5(13.2)$ & $17(17.5)$ & \\
\hline Spending decision $[\mathrm{n}(\%)]^{\mathrm{b}}$ & & & & 0.285 \\
\hline Myself & $10(8.3)$ & $3(8.6)$ & $7(8.2)$ & \\
\hline Partner & $49(40.8)$ & $16(45.7)$ & $33(38.8)$ & \\
\hline Both myself and partner & $51(42.5)$ & $11(31.4)$ & $40(47.1)$ & \\
\hline Other & $10(8.3)$ & $5(14.3)$ & $5(5.9)$ & \\
\hline \multirow[t]{2}{*}{ PROVIDERS } & Total & $\mathrm{PHC}$ & Hospital & \\
\hline & $(n=201)$ & $(n=105)$ & $(n=96)$ & \\
\hline Mean age \pm SD & $39.06 \pm 9.64$ & $38.15 \pm 9.79$ & $40.10 \pm 9.40$ & 0.164 \\
\hline gender [n (\%)] & & & & 0.396 \\
\hline Male & $37(18.4)$ & $17(16.2)$ & $20(20.8)$ & \\
\hline Female & $164(81.6)$ & $88(83.8)$ & $76(79.2)$ & \\
\hline Type of provider [n (\%)] & & & & $<0.001$ \\
\hline Obstetrician/Gynecologist & $6(3.0)$ & $0(0.0)$ & $6(6.3)$ & \\
\hline General Practitioner & $20(10.0)$ & $5(4.8)$ & $15(15.6)$ & \\
\hline Nurse/midwife & $83(41.3)$ & $19(18.1)$ & $64(66.7)$ & \\
\hline Community Health Extension Worker & $79(39.3)$ & $68(64.8)$ & $11(11.5)$ & \\
\hline Community Health Officer & $13(6.5)$ & $13(12.4)$ & $0(0.0)$ & \\
\hline 'using Fisher's exact test & & & & \\
\hline
\end{tabular}




\begin{tabular}{|c|c|c|c|c|c|c|c|c|c|c|}
\hline \multirow[t]{2}{*}{ Structure Attributes } & \multicolumn{3}{|l|}{ Total } & \multicolumn{3}{|c|}{ Primary } & \multicolumn{3}{|c|}{ Secondary } & \multirow[t]{2}{*}{$p$-value } \\
\hline & Mean & SD & $\%$ of total & Mean & SD & $\%$ of total & Mean & SD & $\%$ of total & \\
\hline Data collection tools $(0-3)$ & 2.72 & 0.56 & 90.5 & 2.64 & 0.59 & 88.1 & 2.82 & 0.51 & 94.0 & $0.059^{*}$ \\
\hline Infrastructure $(0-6)$ & 2.78 & 0.18 & 46.3 & 2.45 & 0.21 & 40.8 & 3.26 & 0.29 & 54.3 & 0.012 \\
\hline Guidelines $(0-5)$ & 1.69 & 0.20 & 33.9 & 1.45 & 0.25 & 28.9 & 2.05 & 0.33 & 41.0 & 0.071 \\
\hline General equipment (0-11) & 6.43 & 0.30 & 58.5 & 5.96 & 0.40 & 54.2 & 7.10 & 0.43 & 64.6 & 0.030 \\
\hline Drugs $(0-5)$ & 1.93 & 0.12 & 38.5 & 1.79 & 0.14 & 35.7 & 2.13 & 0.22 & 42.6 & 0.087 \\
\hline Referral mechanisms (0-4) & 2.40 & 0.11 & 60.0 & 2.20 & 0.14 & 54.9 & 2.69 & 0.18 & 67.3 & 0.016 \\
\hline Capacity for HDP-specific services $(0-4)$ & 2.04 & 0.14 & 51.1 & 1.55 & 0.16 & 38.8 & 2.74 & 0.19 & 68.6 & 0.000 \\
\hline Total for inventory/ infrastructure (0-38) & 19.99 & 0.73 & 52.6 & 18.04 & 0.91 & 47.5 & 22.79 & 1.06 & 60.0 & 0.001 \\
\hline Training in last 3 years $(0-6)$ & 1.18 & 0.10 & 19.7 & 1.13 & 0.13 & 18.8 & 1.24 & 0.17 & 20.7 & 0.293 \\
\hline Signs of HDPs $(0-12)$ & 7.66 & 0.22 & 63.8 & 7.21 & 0.32 & 60.1 & 8.14 & 0.30 & 67.8 & 0.019 \\
\hline Management of pre-eclampsia $(0-8)$ & 4.15 & 0.18 & 51.8 & 4.22 & 0.25 & 52.8 & 4.06 & 0.26 & 50.8 & 0.669 \\
\hline Management of severe pre-eclampsia (0-9) & 4.85 & 0.24 & 53.9 & 4.42 & 0.35 & 49.2 & 5.31 & 0.33 & 59.0 & $0.032^{*}$ \\
\hline Management of eclampsia (0-11) & 6.29 & 0.32 & 57.1 & 5.47 & 0.46 & 49.7 & 7.17 & 0.44 & 65.2 & 0.004 \\
\hline Diagnosis of HDPs (0-3) & 1.97 & 0.07 & 65.5 & 1.74 & 0.10 & 58.0 & 2.21 & 0.09 & 73.6 & 0.000 \\
\hline Usage of MgSO4 (0-7) & 1.63 & 0.12 & 23.2 & 1.12 & 0.13 & 15.9 & 2.18 & 0.20 & 31.1 & $0.000^{*}$ \\
\hline Usage of eclampsia prophylaxis $(0-4)$ & 3.81 & 0.04 & 95.1 & 3.89 & 0.05 & 97.4 & 3.71 & 0.06 & 92.7 & $0.994^{*}$ \\
\hline Usage of antihypertensive drugs $(0-5)$ & 0.92 & 0.08 & 18.4 & 0.68 & 0.09 & 13.7 & 1.18 & 0.13 & 23.5 & $0.001^{*}$ \\
\hline Total knowledge/ training score $(0-65)$ & 32.44 & 0.92 & 49.9 & 29.88 & 1.25 & 46.0 & 35.19 & 1.31 & 54.1 & 0.002 \\
\hline
\end{tabular}

\section{Table 4. Average scores for process attributes}

\begin{tabular}{|c|c|c|c|c|c|c|c|c|c|c|}
\hline \multirow[t]{2}{*}{ Process Attribute } & \multicolumn{3}{|c|}{$\begin{array}{l}\text { Total } \\
p \text {-value }\end{array}$} & \multicolumn{3}{|c|}{ Primary } & \multicolumn{3}{|c|}{ Secondary } & \\
\hline & Mean & SD & $\%$ of total & Mean & SD & $\%$ of total & Mean & SD & $\%$ of total & \\
\hline History taking for women with previous pregnancy $(0-6)$ & 3.22 & 0.22 & 53.7 & 2.38 & 0.45 & 39.6 & 3.53 & 0.25 & 58.8 & 0.011 \\
\hline History taking (0-9) & 5.00 & 0.23 & 55.6 & 3.79 & 0.49 & 42.2 & 5.49 & 0.24 & 61.0 & 0.000 \\
\hline Physical exam (0-9) & 5.64 & 0.13 & 62.6 & 5.62 & 0.18 & 62.4 & 5.65 & 0.16 & 62.7 & 0.457 \\
\hline Lab tests $(0-2)$ & 1.04 & 0.08 & 52.2 & 0.85 & 0.16 & 42.3 & 1.13 & 0.09 & 56.3 & 0.060 \\
\hline Documentation (0-2) & 1.46 & 0.06 & 73.0 & 1.31 & 0.12 & 65.4 & 1.52 & 0.07 & 76.0 & 0.063 \\
\hline Disease prevention $(0-12)$ & 8.35 & 0.21 & 69.6 & 8.47 & 0.34 & 70.6 & 8.30 & 0.26 & 69.2 & 0.644 \\
\hline Follow-up/continuation of care $(0-3)$ & 2.35 & 0.08 & 78.3 & 2.44 & 0.12 & 81.2 & 2.31 & 0.10 & 77.1 & 0.752 \\
\hline Total provider/technical skills score $(0-37)^{\text {a }}$ & 17.64 & 0.44 & 47.7 & 15.61 & 0.84 & 42.2 & 18.43 & 0.49 & 49.8 & 0.002 \\
\hline Rapport (0-7) & 6.21 & 0.10 & 88.7 & 6.23 & 0.16 & 89.0 & 6.20 & 0.13 & 88.5 & 0.559 \\
\hline Communication $(0-4)$ & 3.15 & 0.07 & 53.7 & 3.15 & 0.11 & 78.8 & 3.15 & 0.08 & 78.6 & 0.522 \\
\hline Total interpersonal skills score (0-11) & 9.36 & 0.137 & 85.1 & 9.38 & 0.21 & 85.3 & 9.34 & 0.17 & 84.9 & 0.553 \\
\hline
\end{tabular}

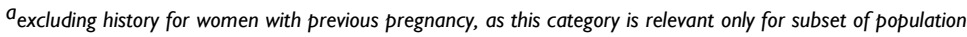




\section{Outcome attributes of care}

Table 5 shows the outcome attributes related to client experiences. Out of a total of 16, PHCs scored significantly lower than hospitals $(45.4 \%$ vs. $55.2 \% ; p=0.004)$. While perceptions of wait times, costs, and satisfaction were not significantly different between facility type, clients at PHCs had significantly lower health literacy than those at hospital health facilities (39.5\% vs. $50.6 \%, p$ $=0.020$ ). Wait-time and time spent with providers were comparable between primary and secondary facilities, but women attending ANC at hospitals reported willingness to pay a higher amount for services (1.28 USD vs. 0.43 USD; $p=0.044$ ) (Table 6). More than half of all women (51.1\%) could not recall any danger signs in pregnancy that would compel them to return to a health provider. Only $5.2 \%$ of clients could name at least 3 danger signs - most commonly severe headache, bleeding or fluids from vagina, and swelling of legs and feet (Table 6). Notably, satisfaction was very high for all facilities; $100 \%$ of respondents in PHCs and $89.4 \%$ of respondents in hospitals reported being satisfied with services received.

The difference observed in overall outcome score between PHCs and hospital facilities was no longer significant after regression adjustment for client age, level of education, and socioeconomic status ( $p=0.189$, [Additional file 2]). Outcome scores for women with a "high" SES were $1.42(95 \% \mathrm{Cl}=0.03,2.80)$ points lower than scores for women with a "low" SES ( $p=$ 0.045). Further adjustment for clustering by specific facility did not quantitatively or qualitatively change the results of the regression analysis.

Table 5 Average Scores for Outcome Attributes

\begin{tabular}{|c|c|c|c|c|c|c|c|c|c|}
\hline \multirow[t]{2}{*}{ Outcome Attribute } & \multicolumn{3}{|l|}{ Total } & \multicolumn{3}{|c|}{ Primary } & \multicolumn{3}{|c|}{ Secondary } \\
\hline & mean & SD & $\%$ of total & mean & SD & $\%$ of total & mean & SD & $\%$ of total \\
\hline Wait and cost $(0-3)$ & 1.58 & 0.09 & 52.6 & 1.34 & 0.17 & 44.7 & 1.67 & 0.11 & 55.7 \\
\hline Satisfaction (0-5) & 3.01 & 0.11 & 60.3 & 2.76 & 0.23 & 55.3 & 3.11 & 0.12 & 62.3 \\
\hline Health literacy $(0-8)$ & 3.80 & 0.20 & 47.5 & 3.16 & 0.33 & 39.5 & 4.05 & 0.24 & 50.6 \\
\hline Total outcome score (0-16) & 8.39 & 0.26 & 52.5 & 7.26 & 0.51 & 45.4 & 8.84 & 0.30 & 55.2 \\
\hline
\end{tabular}

*equal variances not assumed (using F statistic, $p<0.05$ ) 
Table 6 Categorical and Continuous Outcomes

\begin{tabular}{|c|c|c|c|c|}
\hline \multirow[t]{2}{*}{ Key outcome measures of quality of care } & Total & PHC & Hospital & \multirow[b]{2}{*}{ value } \\
\hline & $(n=135)$ & $(n=38)$ & $(n=97)$ & \\
\hline Mean wait time (minutes) $\pm S D$ & $93.8 \pm 78.13$ & $96.41 \pm 81.0$ & $92.85 \pm 77.63$ & 0.839 \\
\hline Wait time $[\mathrm{n}(\%)]^{\mathrm{C}}$ & & & & 0.285 \\
\hline$<1 \mathrm{~h}$ & $33(31.4)$ & $10(37.0)$ & $23(29.5)$ & \\
\hline $1-2 \mathrm{~h}$ & $45(42.9)$ & $8(29.6)$ & $37(47.4)$ & \\
\hline$>2 \mathrm{~h}$ & $27(25.7)$ & $9(33.3)$ & $18(23.1)$ & \\
\hline Mean time spent with provider & $20.4 \pm 17.68$ & $23.6 \pm 19.8$ & $18.8 \pm 16.7$ & 0.415 \\
\hline (minutes) \pm SD Time spent with provider & & & & 0.185 \\
\hline$<5 \mathrm{~min}$ & $9(21.4)$ & $1(7.1)$ & $8(28.6)$ & \\
\hline $5-19 \mathrm{~min}$ & $15(35.7)$ & $7(50.0)$ & $8(28.6)$ & \\
\hline $20-39 \min$ & $10(23.8)$ & $2(14.3)$ & $8(28.6)$ & \\
\hline$>40$ min & $8(19.0)$ & $4(28.6)$ & $4(14.3)$ & \\
\hline First ANC visit [n (\%)] ${ }^{\mathrm{C}}$ & & & & $\begin{array}{l}0.616 \\
a\end{array}$ \\
\hline$<15$ weeks & $28(24.1)$ & $5(17.9)$ & $23(26.1)$ & \\
\hline 15-24 weeks & $41(35.3)$ & $9(32.1)$ & $32(36.4)$ & \\
\hline 25-30weeks & $28(24.1)$ & $9(32.1)$ & $19(21.6)$ & \\
\hline$>30$ weeks & $19(16.4)$ & $5(17.9)$ & $14(15.9)$ & \\
\hline Mean amount willing to pay for services (USD) ${ }^{b} \pm$ SD & $1.06 \pm 1.59$ & $0.43 \pm 0.40$ & $1.28 \pm 1.79$ & 0.044 \\
\hline Amount willing to pay for services $[\mathrm{n}(\%)]^{\mathrm{C}}$ & & & & 0.741 \\
\hline$<0.27$ USD & $6(19.4)$ & $2(25.0)$ & $4(17.4)$ & \\
\hline $0.27-1.37$ USD & $18(58.1)$ & $5(62.5)$ & $13(56.5)$ & \\
\hline$>1.37$ USD & $7(22.6)$ & $1(12.5)$ & $6(26.1)$ & ח11 \\
\hline Satisfaction $[\mathrm{n}(\%)]^{\mathrm{C}}$ & & & & a \\
\hline Satisfied & $107(92.2)$ & $31(100.0)$ & $76(89.4)$ & \\
\hline Somewhat or not satisfied & $9(7.8)$ & $0(0.0)$ & $9(10.6)$ & \\
\hline Knowledge of danger signs in pregnancy [ $(\%)]$ & & & & $\begin{array}{l}0.504 \\
a\end{array}$ \\
\hline 0 & $69(51.1)$ & $21(55.3)$ & $48(49.5)$ & \\
\hline 1 & $30(22.2)$ & $10(26.3)$ & $20(20.6)$ & \\
\hline 2 & $29(21.5)$ & $5(13.2)$ & $24(24.7)$ & \\
\hline 3 & $7(5.2)$ & $2(5.3)$ & $5(5.2)$ & \\
\hline
\end{tabular}

${ }^{a}$ using Fisher's exact test

${ }^{b}$ I USD $=364.50 \mathrm{NGN}$

'effective sample size does not equal total sample due to missing data 


\section{Discussion}

This analysis provides a comprehensive cross-sectional overview of the quality of ANC services provided across the six geo-political zones of Nigeria and explores how quality differs between PHC and hospitals. In general, it was found that the quality of ANC provided by Nigerian healthcare facilities is sub-par and does not meet many of the WHO-required elements for adequate ANC. All elements of quality of care, except for interpersonal skills, obtained a cumulative score of below 55\%. Furthermore, PHCs scored lower than hospitals in most domains. Despite these relatively low scores for quality of care, self-reports of client satisfaction were very positive.

\section{Structure}

Facilities captured in this study obtained very low scores for inventory and infrastructure - below half of the tools, recent guidelines, equipment, drugs, and mechanisms deemed necessary to perform antenatal services. Within general equipment, only three quarters of facilities had operational sphygmomanometers for measuring blood pressure, and less than half had dipsticks for proteinuria testing. These numbers are concerning, especially regarding the important role they play in diagnosing pre- eclampsia and eclampsia [22].

PHCs scored significantly lower than hospitals in almost all aspects of inventory and infrastructure. Ademiluyi and Aluko Arowolo [23] have written on the unique infrastructural challenges faced by PHCs, citing Nigeria's colonial history and its implications on the evolution and distribution of healthcare resources as the root cause. To this day, the bulk of public healthcare dollars are spent in secondary facilities, as is government spending on general infrastructure [24]. While all levels of facilities must meet standards for accreditation, secondary level facilities have much stricter infrastructure requirements, including those for operating theatre, pharmacy, laboratory, and personnel capacities [25]. Despite their limited capacity to treat pregnant women, PHCs play a vital role in advising women on danger signs of obstetric complications, detecting these complications (including pre-eclampsia and eclampsia) early and referring women to general or specialist hospitals in a timely manner (often following initial stabilizing care, such as administration of loading dose of MgSO4) [26]. However, PHCs likely face 
challenges in efficiently getting patients to hospitals in the case of emergencies, as this study reveals that only a quarter have any ambulatory transport systems.

Other research has found a similar lack of infrastructure in both primary and secondary facilities in Nigeria. Kress et al. [12] reported on a "general shortage of drugs and supplies" that likely are the result of segmented supply chains and financial constraints placed on Local Government Authorities (LGAs) who are responsible for allocating resources to PHCs. They report that as much as $95 \%$ of funding must be allocated to staff salaries at the expense of adequate drugs, supplies, and maintenance. Oyekale [11] similarly found that tools and drugs essential for ANC including sphygmomanometers, thermometers, stethoscopes, magnesium sulfate, folic acid, and calcium gluconate were unavailable or dysfunctional in as many as $87 \%$ of facilities studied.

Provider knowledge and training received the lowest overall scores, with PHCs scoring significantly lower than hospitals. This finding is not entirely unexpected given the presence of lower level staff at PHCs compared to secondary facilities. Indeed, regression analysis shows that provider knowledge scores are dependent on the type of provider, (with obstetricians/gynecologists scoring at least 12 points higher than the next best cadre - general practitioners). This is not to say that lower cadre health workers cannot be adequately trained on critical components of ANC, as has been demonstrated in previous research [27-30]. Despite existing evidence that shows task-shifting and training of lower-cadre health-care workers is an important strategy for low-income countries, only $12.2 \%$ of providers in this study reported having received any training in the past 3 years.

\section{Process}

For both PHCs and hospitals, scores for providers' skills were moderate to low. Providers were relatively successful in the realm of 'follow-up/ continuation of care' (most clients were observed being instructed to come for a follow-up appointment and told where to go for follow-up). However, a smaller percentage were given a written reminder of when to come or encouraged to re- turn in the case of emergency. It is well-documented that Nigerian women attend fewer ANC consultations than is recommended for healthy pregnancy and child- birth [8]. Providing 
written reminders may be a simple strategy to improve rates of ANC attendance. Previous research in Zanzibar has shown the effects of follow-up reminders (via SMS) to be beneficial in not only the number of ANC visits attended, but also in the percentage of women with antenatal complications identified and referred [31]. In 2015, Fagbamigbe and Idemudia [16] studied ANC quality in Nigeria and reported on the eight nationally recommended 'critical components' of ANC (blood pressure, iron supplements, blood sample, urine sample, tetanus injections, danger signs, HIV tests, malaria prophylaxis). As in our study, the most common of these eight components practiced were blood pressure measurement and provision of iron/folic acid supplements. Compared with those in Fagbamigbe and Idemudia, the participants in our study reported higher frequency of HIV testing (78.5\% vs. $41.7 \%)$ and provision of malaria prophylaxis (77.8\% vs. $40.1 \%$ ), and lower frequency of urine testing ( $28.1 \%$ vs. $81.9 \%)$. Furthermore, process scores may be an optimistic interpretation of the provider's skill in these areas; although the data tells us that the actions were taken, it does not specify the degree to which they were correctly completed. For example, there are many sources of inaccuracy for blood pressure measurement including patient, device, procedure, and observer-related sources [32]. Previous research has identified blood pressure inaccuracy as a challenge in hospitals in developing nations, and especially among providers with lower qualifications such as nurses and CHOs [33, 34].

Provider interpersonal skills, as assessed by direct observation of their interaction with clients, scored highly. Audio and visual privacy was the only interpersonal indicator in which PHCS performed worse than secondary facilities; this is understandable given the relatively little space and busy atmosphere of PHCs. Babatunde et al. [35] found similarly positive results among PHC users, with the ideal interpersonal skills being displayed in $71-91 \%$ of healthcare interactions.

\section{Outcome}

Low scores in health literacy (dissemination, communication, and explanation of consultation results) were reflected in clients' poor recall of danger signs of pregnancy. Hospitals did significantly better in this regard than PHC, possibly because of better-trained staff, or more educated clients. Gaining sufficient and adequate information about one's health and potential complications from a care provider is important during the antenatal period and is associated 
with improved ANC attendance and pregnancy outcomes [36-38]. Health literacy is critical to patient empowerment, and accurate information from an informed provider is especially valuable when the alternative might be informal education, misrepresentation, or mythology. Despite the low scores received for structural and process attributes, as well as moderate perceptions of wait time and cost, all facilities scored extremely well in terms of client satisfaction. This result is encouraging, as each new interaction with the health system presents an additional opportunity for HDPs to be detected and managed. However, perception of care must be interpreted cautiously, as low-income and low-education women can be uncritical of the healthcare they receive or base it on the provider's interpersonal skills rather than their competence [17, 39, 40]. Only a quarter of clients attended an ANC visit before 15 weeks gestational age, despite the WHO's recommendations for an initial contact within 12 weeks of pregnancy. This is especially important for first time mothers, and to ensure early detection of HDPs, HIV, and anemia [1].

In 2016, the WHO released "Standards for Improving Quality of Maternal and Newborn Care in Health Facilities" a document which outlines 8 domains of quality of care that should be assessed, improved, and monitored within the health system [41]. These range from the appropriate use of data, to effective and compassionate communication with women and their families, to ap- propriate physical environment and competent, motivated staff. Furthermore, quality antenatal care should result in a "positive pregnancy experience", defined as not only maintaining a healthy pregnancy for the mother and baby, but "having an effective transition to positive labor and birth", and "achieving positive motherhood (including maternal self-esteem, competence and autonomy)" [1]. In accordance with these standards, our study shows that improvements to ANC must be holistic and encompass not just the facility, but also provider know- ledge and practices. It must also involve community mobilization to educate on the importance of ANC and improve maternal health literacy. In an environment where resources are limited and there are competing interests in terms of health priorities, solutions must be innovative and easy to adopt, especially for PHCs. This may include mobile training and decisionmaking tools, such as the m4Change application created as part of the Nigerian government's 
Saving One Million Lives Initiative [29]. Policy that mandates regular training refreshers around ANC skills could help to close knowledge gaps. Improvements should be evidence-based and outcomes- oriented, meaning that they are measured using outcome indicators such as the number of women who have at least four ANC visits, tetanus protection at birth, or the proportion of women with a written birth and emergency plan at 37 weeks of pregnancy [42]. This research comes during the development of a new National Health Act, in which the Federal Government of Nigeria has committed to "reactivate" and "revitalize" the country's PHCs. This has begun with the inauguration of an inter- agency Supply Chain Committee, which will act as the implementation partner for the PHC revitalization agenda [43]. This research may therefore serve as a framework for which major gaps in PHC quality, specifically as it pertains to ANC and the ability to manage HDPs, require immediate attention.

\section{Limitations}

There are several limitations to our approach in the identification of indicators that adequately assess quality of ANC, and more specifically its capacity to detect and manage HDPs, as there is little consensus or predetermined framework for doing so. Quality and abundance of indicators are further limited in that they only include aspects of care that should be provided at every ANC consultation (data is cross-sectional and does not encompass multiple ANC visits for a single patient). Indicators used for "outcome" are limited in that they do not capture postnatal outcomes such as birth success, incidence of disease, survival, or long-term health conditions.

However, they do measure outcomes of the consultation itself by showing how much knowledge and satisfaction a woman gained from her ANC visit, and how likely she would be to return. Facilities and clients were selected purposively, leading to potential selection bias and lack of external generalizability. However, because it was conducted across the country (and across all geo-political zones) - it reflects the population of most women attending ANC in Nigerian facilities. As with all studies that rely on interview-based data collection, this study is limited by interviewer and respondent bias. To mitigate this, research assistants were trained on interviewing and data collection techniques. Additionally, interview sources were supplemented with more objective data sources - facility and ANC visit observational checklists. 


\section{Conclusion}

We identified three major obstacles to quality ANC in Nigeria and its ability to detect and manage HDP: 1) inadequate infrastructure, particularly in the lack of facility guidelines, drug stock, referral mechanisms, and general equipment including sphygmomanometers and urine dipsticks; 2) very inadequate provider knowledge, due to infrequent training updates and low understanding of the use of drugs to prevent and treat HDP, and 3) poor communication of health results to clients, resulting in low maternal health literacy. These issues are generally exacerbated in PHCs vs. hospitals due to lower level cadres of healthcare workers and inadequacy of funding for essential supplies, equipment, and infra- structure. Future research may wish to explore additional elements of the quality of ANC in Nigeria and how it impacts birth and postnatal outcomes. This includes outcomes of eclampsia seizures, likelihood and success of cesarean section following PE/E indication, days of hospitalization, and admission to intensive care.

\section{Acknowledgements}

We thank all clients and healthcare providers who participated in the landscape analysis, and staff at the affiliated facilities. We also thank the Ending Eclampsia team based in Abuja, Nigeria, comprised of Gloria Adoyi, Roli Akpolo, Emmanuel Nwala, and Charles Nwaigwe. Additionally, Dr. Pooja Sripad, Dr. Timothy Abuya, and Dr. George Eluwa (Population Council) provided statistical, methodological, and general support.

\section{Authors' contributions}

$\mathrm{CW}, \mathrm{SI}$, and KK contributed to development of the protocol, data collection, and reviewing the manuscript. AS was responsible for data cleaning, analysis, and writing the manuscript. All authors read and approved the final manuscript.

\section{Funding}

Support for this paper was made possible by the generous support of the American people through the United States Agency for International Development (USAID) under the terms of USAID APS-OAA-13-000005. The contents of this paper are the sole responsibility of the authors and do not necessarily reflect the views of USAID or the United States Government. Apart from requesting that the study cover Nigeria's six geo-political zones, the funding body did not play a role in specific site selection, design, execution, analysis or write-up of this study. 


\section{Availability of data and materials}

The datasets from the current study are available from the corresponding author on reasonable request.

\section{Ethics approval and consent to participate}

Ethical approval was granted by the Population Council's Institutional Review Board (IRB) in New York City (p. 693), the National Health Research Ethics Committee (NHREC) of the Federal Ministry of Health (FMoH), Nigeria (NHREC/01/01/2007-20/06/2015b), and the ethics committees of all the participating states within Nigeria. Written, informed consent was obtained from each participant, with emphasis that the study was voluntary and confidential. Data collectors were trained on ethical conduct.

\section{Consent for publication}

Not applicable.

\section{Competing interests}

The authors declare that they have no competing interests.

\section{Author details}

${ }^{1}$ Dalla Lana School of Public Health, Toronto, Canada. ${ }^{2}$ Population Council, Abuja, Nigeria. ${ }^{3}$ Population Council, New York, USA. ${ }^{4}$ Population Council, Washington, USA. 


\section{References}

1. World Health Organization. WHO recommendations on antenatal care for a positive pregnancy experience: World Health Organization; 2016.

2. Oladapo O, Adetoro O, Ekele B, Chama C, Etuk S, Aboyeji A, et al. When getting there is not enough: a nationwide cross-sectional study of 998 maternal deaths and 1451 nearmisses in public tertiary hospitals in a low- income country. BJOG. 2016;123(6):928-38.

3. Mammaro A, Carrara S, Cavaliere A, Ermito S, Dinatale A, Pappalardo EM, etal. Hypertensive disorders of pregnancy. J Perinat Med. 2009;3(1):1.

4. Oyerinde K. Can antenatal care result in significant maternal mortality reduction in developing countries. J Community Med Health Educ. 2013; 3(2):2-3.

5. WHO. World health statistics 2014. Geneva: World Health Organization; 2014.

6. UNICEF. Antenatal Care - UNICEF data. https://data.unicef.org/topic/ maternalhealth/antenatal-care/\#. Accessed 23 May 2017.

7. Omer K, Afi NJ, Adamu M, Malami SA, Oyo-Ita A, Cockcroft A, et al. Seeking evidence to support efforts to increase use of antenatal care: a cross- sectional study in two states of Nigeria. BMC pregnancy childbirth. 2014; 14(1):380.

8. Mallick L, Tukur D, Kerry M. Trends in maternal health in Nigeria, 2003-2013. DHS Further Analysis Reports. 2016; (102).

9. Donabedian A. Evaluating the quality of medical care. The Milbank memorial fund quarterly. 1966;44(3):166-206. 
10. Ayanian JZ, Markel H. Donabedian's lasting framework for health Care quality. N Engl J Med. 2016;375(3):205-7.

11. Oyekale AS. Assessment of primary health care facilities' service readiness in Nigeria. BMC Health Serv Res. 2017;17(1):172.

12. Kress DH, Su Y, Wang H. Assessment of primary health Care system performance in Nigeria: using the primary health Care performance. Indicator conceptual framework. Health Systems \& Reform. 2016;2(4):302-18.

13. Warren CE, Ishaku S, Oginni A, Adoyi G, Kirk KR, Dempsey A. Landscape analysis of preeclampsia/eclampsia in Nigeria. Abuja: Population Council; 2015.

14. Balogun O. Patients perception of quality of antenatal service in four selected private health facilities in Ilorin, Kwara state of Nigeria. Niger Med Pract. 2007;51(4):80-4.

15. Oladapo OT, Iyaniwura CA, Sule-Odu AO. Quality of antenatal services at the primary care level in Southwest Nigeria. Afr J Reprod Health. 2008;12(3):71-92.

16. Fagbamigbe AF, Idemudia ES. Assessment of quality of antenatal care services in Nigeria: evidence from a population-based survey. Reprod Health. 2015;12(1):88.

17. Warren CE, Abuya T, Kanya L, Obare F, Njuki R, Temmerman M, et al. A cross sectional comparison of postnatal care quality in facilities participating in a maternal health voucher program versus non-voucher facilities in Kenya. BMC pregnancy childbirth. 2015;15(1):153.

18. Bruce J. Fundamental elements of the quality of care: a simple framework. Stud Fam Plan. 1990;21(2):61-91. 
19. Hulton L, Matthews Z, Stones RW. Applying a framework for assessing the quality of maternal health services in urban India. Soc Sci Med. 2007;64(10): 2083-95.

20. Vyas S, Kumaranayake L. Constructing socio-economic status indices: how to use principal components analysis. Health Policy Plan. 2006;21(6):459-68.

21. StataCorp. 2017. STATA (release 15) statistical software. College Station, TX: StataCorp LLC.

22. WHO. WHO recommendations for prevention and treatment of pre- eclampsia and eclampsia. Geneva: World Health Organization; 2011.

23. Ademiluyi IA, Aluko-Arowolo SO. Infrastructural distribution of healthcare services in Nigeria: an overview. J Geog Reg Plann. 2009;2(5):104-10.

24. Central Bank of Nigeria. Annuals report and statement of account for the year-ended 31st December $2005 . \quad$ https://www.cbn.gov.ng/OUT/2010/ PUBLICATIONS/REPORTS/RSD/Link\%20Files/INTERACTIVE\%20ANNUAL

25. \%2OREPORT\%2OFOR\%20THE\%2OYEAR\%20ENDED\%20DECEMBER\%202009. pdf. Accessed 17 Aug 2017.

26. National Health Insurance Scheme. National Health Insurance Scheme Operational Guidelines. http://www.dhmlnigeria.com/downloads/NHIS_ OPERATIONAL_GUIDELINES(Revised).pdf. Accessed 17 Aug 2017.

27. Oguntunde O, Charyeva Z, Cannon M, Sambisa W, Orobaton N, Kabo IA, et al. Factors influencing the use of magnesium sulphate in pre-eclampsia/ eclampsia management in health facilities in northern Nigeria: a mixed methods study. BMC Pregnancy Childbirth. 2015;15(1):130. 
28. Fulton BD, Scheffler RM, Sparkes SP, Auh EY, Vujicic M, Soucat A. Health workforce skill mix and task shifting in low income countries: a review of recent evidence. Hum Resour Health. 2011;9(1):1.

29. Ishaku S, Ahonsi B, Tukur J, Ayodeji O. Attrition from care after the critical phase of severe pre-eclampsia and eclampsia: insights from an intervention with magnesium sulphate in a primary care setting in northern Nigeria. Health. 2013;5(09):1461.

30. McNabb M, Chukwu E, Ojo O, Shekhar N, Gill CJ, Salami H, et al. Assessment of the quality of antenatal care services provided by health workers using a mobile phone decision support application in northern Nigeria: a pre/post- intervention study. PLoS One. 2015;10(5): e0123940.

31. Ameh CA, Kerr R, Madaj B, Mdegela M, Kana T, Jones S, et al. Knowledge and skills of healthcare providers in sub-Saharan Africa and Asia before and after competency-based training in emergency obstetric and early newborn care. PLoS One. 2016;11(12): e0167270.

32. Lund S, Nielsen BB, Hemed M, Boas IM, Said A, Said K, et al. Mobile phones improve antenatal care attendance in Zanzibar: a cluster randomized controlled trial. BMC Pregnancy Childbirth. 2014;14(1):29.

33. Kallioinen N, Hill A, Horswill MS, Ward HE, Watson MO. Sources of inaccuracy in the measurement of adult patients' resting blood pressure in clinical settings: a systematic review. J Hypertens Manag. 2017;35(3):421.

34. Ahmed MEBK. Knowledge of blood pressure measurement among a teaching hospital staff in a developing nation. J Hum Hypertens. 1997; 11(8):495. 
35. Ayodele O, Okunola O, Akintunde A, Sanya E. End digit preference in blood pressure measurement in a hypertension specialty clinic in Southwest Nigeria: cardiovascular topics. Cardiovasc J Afr. 2012;23(2):85-9.

36. Babatunde OA, Aiyenigba E, Awoyemi OA, Akande TM, Musa OI, Salaudeen AG, et al. Primary health Care Consumers' perception of quality of Care and its determinants in north-Central Nigeria. J Asian Sci Res. 2013;3(7):775.

37. Kohan S, Ghasemi S, Dodangeh M. Associations between maternal health literacy and prenatal care and pregnancy outcome. Iran J Nurs Midwifery Res. 2008; 12:4.

38. Simkhada B, ERv T, Porter M, Simkhada P. Factors affecting the utilization of antenatal care in developing countries: systematic review of the literature. J Adv Nurs. 2008;61(3):244-60.

39. Mojoyinola J. Influence of maternal health literacy on healthy pregnancy and pregnancy outcomes of women attending public hospitals in Ibadan, Oyo state, Nigeria. Afr Res Rev. $2011 ; 5: 3$.

40. Porter $M$, Macintyre S. What is, must be best: a research notes on conservative or deferential responses to antenatal care provision. Soc Sci Med. 1984;19(11):1197-200.

41. Fawole AO, Okunlola MA, Adekunle AO. Clients' perceptions of the quality of antenatal care. J Natl Med Assoc. 2008;100(9):1052-8.

42. WHO. Standards for improving quality of maternal and newborn care in health facilities. Geneva: World Health Organization; 2016. 
43. Lincetto O, Mothebesoane-Anoh S, Gomez P, Munjanja S. Antenatal care. In: Lawn J, Kerber K, editors. Opportunities for Africa's newborns: practical data, policy and programmatic support for newborn care in Africa. Capetown: Mills Lotho; 2006. p. 55-62.

44. Stewart S, Charles J. Nigeria's President Commits to Revitalizing 10,000 Primary Health Care Centers. North Bethesda; USAID; 2017. 




\section{Chapter 3}

Adherence to Guidelines in Postpartum Management of Hypertensive Disorders in Pregnancy in Nigeria: A Multi-centre Study

\section{Salisu M. Ishaku}

Lawal Oyeneyin

Onyebuchi Azubuike

Innocent Agbo

Lamaran Dattijo

Aminu Baffah

Gbenga Kayode

Olorunfemi Owa

Patience Odusolu

Karima Tunau

Jamilu Tukur

Charlotte E. Warren

Hannifa Abubakar

Musa Abdulkarim

Arie Franx

Diederick Grobbee

Joyce Browne

First and second authors contributed equally

Submitted for publication in the Journal of Health Systems and Reform, reviewers' comments have been addressed, awaiting final decision 


\section{Abstract}

\section{Background}

Hypertensive disorders in pregnancy (HDP) are a leading cause of maternal mortality in low- and middle-income countries (LIMCS) like Nigeria. Guidelines for postpartum management are available and expected to be optimally utilized.

\section{Objective}

This study aimed to determine adherence to these guidelines in selected Nigerian hospitals.

\section{Methods}

This study is nested in a cohort of women with HDP who delivered in eight tertiary hospitals across Nigeria between October 2017 and June 2018. Nine weeks after delivery, their case notes were evaluated on 14 pre-specified indicators and supplemented with questionnaire-based interviews. The level of adherence to the guidelines was determined using descriptive analyses including frequencies, percentages, means and standard deviations as well as charts.

\section{Results}

Of the 366 participants, 33 (9\%), 75 (20\%), 200 (55\%) and 58 (16\%) had chronic hypertension, gestational hypertension, pre-eclampsia and eclampsia, respectively. Only about a third of all the women with HDP had their blood pressure measured between postpartum days three to five (7\%, $16 \%, 60 \%$ and $17 \%$ distributed respectively as above). Similarly, a third of those with persistent hypertension $(\geq 150 / 100 \mathrm{mmHg}$ ) were not on antihypertensive medications within eight weeks postpartum. In addition, $37 \%$ and $42 \%$ of participants were not counselled on contraceptives use and early antenatal visit in future pregnancies, respectively. Among those with pre-eclampsia/eclampsia, 93\% were not offered screening for risk of thrombo-prophylaxis postpartum. Although all women with pre-eclampsia/eclampsia in this study remained hypertensive two weeks after discharge, only $24 \%$ were offered medical review. Overall, only $58 \%$ and $44 \%$ of all HDPs and pre-eclampsia/eclampsia-specific indicators were satisfied, respectively. 


\section{Conclusion}

Level of adherence to guidelines on postpartum management of HDP in tertiary hospitals in Nigeria is poor. It is recommended that institutionalization of these guidelines be prioritized and linked to the entire continuum of care from pre-conception, pregnancy, childbirth through longer term postpartum care.

Key words: Quality of care, hypertensive disorders in pregnancy, postpartum management, Nigerian tertiary hospitals 


\section{Introduction}

Hypertensive disorders in pregnancy (HDP) complicate $5.2 \%$ to $8.2 \%$ of pregnancies, worldwide ${ }^{1}$. They affect one in ten pregnancies in sub-Saharan Africa (SSA) with higher prevalence in Central and West African regions ${ }^{2}$. They are also responsible for 70,000 maternal deaths globally; killing a woman every 11 minutes $^{1,2}$. A 2015 survey sponsored by the World Health Organization (WHO) and conducted in 42 tertiary hospitals across Nigeria revealed HDP as the leading cause of maternal deaths accounting for $23 \%^{3}$. Other reviews showed that a significant proportion of deaths from severe cases of HDP occurred in the postpartum period ${ }^{4-6}$. These deaths were mostly related to lack of adherence to recommended practices, especially poor control of postpartum blood pressure. In addition, about $25 \%$ of women with HDP, particularly those with a severe condition, experience a deterioration of end organs functions during puerperium which is the period spanning six to eight weeks after delivery during which pregnancy changes revert ${ }^{7}$. More studies also showed that pre-eclampsia (PE) occurring early in pregnancy ( $<34$ weeks of gestation), presenting in its severe form or persisting with proteinuria beyond three to six months after delivery could develop into chronic diseases such as hypertension, stroke, diabetes mellitus, renal and ischemic heart diseases as well as increased risk for venous thromboembolism (VTE) ${ }^{8-}$ 10.

Lack of capacity, knowledge and skills among lower-level health care providers for early detection, monitoring, managing and or referral of severe forms of HDP such as pre-eclampsia and eclampsia, is implicated as contributing to these avoidable deaths $\mathrm{s}^{3,11-12}$. In addition to that of antenatal and intrapartum care, maternal outcomes in HDP are influenced by the quality of postpartum care. Specifically, women with HDP need monitoring in the immediate postpartum period and beyond. Towards this end, international guidelines are widely available to guide healthcare providers ${ }^{1,9,13-15}$. Implementation of these guidelines enables strict monitoring of signs and symptoms, prompt identification and management of postpartum complications such as eclampsia, cerebrovascular accident, thromboembolism and renal impairment. The Federal Ministry of Health of Nigeria in collaboration with Population Council pioneered the development of a service delivery manual on the management of women with HDP, including during 
immediate postpartum period relevant for use by the cadre of health workers at primary and secondary levels of care ${ }^{16}$.

To date, no study has examined the level of adherence to clinical guidelines in postpartum management of HDP in sub-Saharan Africa. A report on quality of care assessment for women and their babies following HDP is available for Bangladesh ${ }^{17}$. Tertiary hospitals being referral centers are expected to provide the best form of quality of health care services pertaining to all medical conditions, including HDP. This study, therefore, aimed to determine the level of adherence to standard guidelines in postpartum management of HDP at selected tertiary hospitals in Nigeria.

\section{Methodology}

\section{Study Design}

This study was nested in a cohort of women with HDP who delivered and received postpartum care at tertiary health facilities in Nigeria, including a minimum of five days as inpatients. After discharge, they were then expected to be followed up on out-patient basis till full recovery. Data collection was conducted at nine weeks after delivery between October 2017 and June 2018. The choice of ninth week postpartum as data collection point was to allow the usual six to eight weeks postpartum visit period to elapse. This enabled determining whether recommended postpartum care services were provided or not.

\section{Study setting}

The study was conducted in eight tertiary hospitals across the six geo-political zones of Nigeria. The hospitals were purposefully selected to reflect diversity in the country in terms of ethnicity and socio-economic status. The following states (and hospitals) participated: Bauchi State (Abubakar Tafawa Balewa University Teaching Hospital), Cross River State (University of Calabar Teaching Hospital), Ebonyi State (Federal Teaching Hospital Abakaliki), Kogi State (Federal Medical Center, Lokoja), Kano State (Aminu Kano Teaching Hospital), Ondo State (Mother and Child Hospital Akure and University of Medical Sciences Teaching Hospital) and Sokoto State (Usman Danfodio University Teaching Hospital). 
Due to the geographical spread, women who received care in these facilities were considered a good representation of the diversity in the country. The selected tertiary health facilities were high-volume sites with well-functioning ante- and postnatal clinics, delivery rooms as well as laboratory services with combined annual deliveries averaging 38,400.

\section{Participants}

Women 18 years and above, who delivered and received postpartum care at these facilities (regardless of where they received antenatal care) as well as a diagnosis of HDP were eligible for inclusion. No exclusion criteria were applied.

\section{Exposure variables}

The main exposure of interest was the presence of any of the HDP sub-types including chronic hypertension, gestational hypertension and pre-eclampsia/eclampsia as defined by the International Society for the Study of Hypertension in Pregnancy (ISSHP) ${ }^{13}$ :

- Chronic hypertension in pregnancy: Any hypertension ( $\mathrm{BBP} \geq 140$ and or $\mathrm{dBP} \geq 90 \mathrm{mmHg}$ ) that was present pre-pregnancy or diagnosed before the 20th week of pregnancy

- Gestational hypertension: Any hypertension ( $\mathrm{BBP} \geq 140$ and or $\mathrm{dBP} \geq 90 \mathrm{mmHg}$ ) that appears for the first time after the 20th week of pregnancy without significant proteinuria, fetal growth restriction or evidence of biochemical and haematological abnormalities

- Pre-eclampsia/eclampsia: Any hypertension (sBP $\geq 140$ and or $\mathrm{dBP} \geq 90 \mathrm{mmHg}$ ) that appears for the first time after the 20th week of pregnancy associated with significant proteinuria, fetal growth restriction or any biochemical and haematological abnormalities. If a client with pre-eclampsia experiences tonic-clonic convulsions in the absence of a neurological cause, it was regarded as eclampsia

- Significant protein in urine (proteinuria): Protein excretion in urine of $\geq 2++$ using urine dipsticks measurement

\section{Outcome variables}

The level of adherence was assessed by pre-specified indicators that were selected from standard guidelines on postpartum management for women with HDP. The guidelines include those developed by the Federal Ministry of Health of Nigeria ${ }^{16}$, the International Federation of Gynecologists and Obstetricians (FIGO) ${ }^{1}$, the National Institute for Health and Care Excellence 
$(\mathrm{NICE})^{14}, \mathrm{ISSHP}^{13}, \mathrm{WHO}^{15}$ and the Royal College of Obstetricians \& Gynecologists (RCOG) ${ }^{9}$. Although there is no consensus across guidelines, the 14 study indicators were based on agreed minimal care components for women with HDP during postpartum period. Only healthcare services that were available within Nigeria's health system were selected for evaluation. These include:

- Daily blood pressure (BP) measurement in the first two days postpartum for women with chronic and gestational hypertension, and measurement of BP four times a day in the first two days postpartum for women with pre-eclampsia ${ }^{13-14,16}$

- Measurement of BP, at least, once between day 3 and 5 after birth in women with all forms of HDP ${ }^{1,14,16}$

- Whether women with pre-eclampsia were asked about severe headache or epigastric pain any time $\mathrm{BP}$ is measured ${ }^{13-14,16}$

- Whether health care providers targeted a BP value of less than $140 / 90 \mathrm{mmHg}$ after delivery in women with HDP ${ }^{15-16}$

- Continuation of anti-hypertensive treatment for women who remained hypertensive ${ }^{14-15}$

- Offering (specialist) medical review for women with gestational hypertension and preeclampsia who remain on anti-hypertensive treatment two weeks after discharge from hospital ${ }^{14}$

- Offering (specialist) medical review to women with any form of HDP at between six to eight weeks postpartum ${ }^{14,16}$

- Offering specialist assessment for women with gestational hypertension and preeclampsia who still required anti-hypertensive treatment six to eight weeks after delivery ${ }^{14}$

- For women who had pre-eclampsia, whether they had their platelets, transaminases and serum creatinine measured forty-eight to seventy-two hours after delivery ${ }^{13-14}$

- If women with pre-eclampsia had urine protein estimation using urine dipsticks at six to eight weeks after birth ${ }^{14}$

- If desirous for another pregnancy, women with pre-eclampsia were advised to achieve and keep a BMI within a healthy range $(18.5-24.9 \mathrm{~kg} / \mathrm{m} 2)^{14}$ 
- Had PE/E in isolation or with additional risk factor for thrombosis (e.g. Age > 35, obesity and parity $\geq 3$, etc.), and received thrombo-prophylaxis at any point after delivery ${ }^{9}$

- If women were counseled to attend ANC early in next pregnancy ${ }^{16}$

- If women were counseled on family planning at any time before or after delivery ${ }^{16}$

In addition, information captured on participants' obstetrics and demographic characteristics included age, parity, body mass index (BMI) and healthcare facilities were collected.

\section{Data source/measurements}

Participants' health records were evaluated, and data retrieved, supplemented with questionnaire-based interviews conducted by trained research assistants.

\section{Sample size}

The required sample size was 73 , including a $10 \%$ potential non-response. This was derived from a $5 \%$ prevalence of HDP among pregnant women ${ }^{1-2}$. This study, however, evaluated 366 participants.

\section{Risk of bias}

To minimize measurement errors and misclassification bias, case identification and outcomes assessment were performed by specially trained midwives/nurses and medical officers using standard diagnostic criteria. The information retrieved were entered into the electronic data capturing platform by trained research assistants as soon as the data were obtained to reduce the incidence of missing data. The hard copies of all records were retained as source documents at the facilities for future reference when necessary.

\section{Data Analysis}

Descriptive statistics were performed to describe the obstetrics and demographic characteristics of the participants. The level of adherence to standard guidelines in postpartum management of HDP was expressed in terms of frequencies, percentages, mean and standard deviations. A 
compound bar chat was used to visualize trends in adherence to the management of blood pressure during the postpartum period. The data was analyzed using SPSS IBM version 25.0.

\section{Results}

Out of 366 women with HDP evaluated at nine weeks postpartum, 33 (9\%), 75 (20\%), 200 (55\%) and 58 (16\%) had chronic hypertension, gestational hypertension, pre-eclampsia and eclampsia, respectively. The majority was multiparous $(55 \%)$, relatively overweight with mean $\mathrm{BMI}$ of $28.8 \mathrm{~kg} / \mathrm{m}^{2}(\mathrm{SD}=7.7)$ and a mean maternal age of 28.7 years $(S D=6.4)$. About $40 \%(145)$ of all women did not receive antenatal care, and among those who did, only $3 \%$ (12) registered within the first trimester. The majority received the diagnosis of HDP $\geq 34$ weeks gestation ( $n=235$, $64 \%)$, delivered at term $(n=219,60 \%)$ and had a caesarean section $(n=195,53 \%)$. Details of other obstetric and demographic variables are shown in Table 1.

Table 1: Baseline characteristics of study participants

\begin{tabular}{|c|c|c|c|c|c|c|}
\hline \multirow[t]{2}{*}{ Variables } & \multicolumn{2}{|c|}{ Records reviewed ( $\mathrm{N}=366$ ) } & \multirow{2}{*}{$\begin{array}{l}\text { Chronic } \\
\text { hypertension }\end{array}$} & \multirow{2}{*}{$\begin{array}{l}\text { Gestational } \\
\text { hypertension }\end{array}$} & \multirow[t]{2}{*}{ Pre-eclampsia } & \multirow[t]{2}{*}{ Eclampsia } \\
\hline & Number (\%) & Mean (SD) & & & & \\
\hline HDP sub-types $n(\%)$ & & & $33(9.0)$ & $75(20.5)$ & $200(54.6)$ & $58(15.8)$ \\
\hline Mean age (SD)* & & $28.7(6.4)$ & $35.2(6.8)$ & $33.3(28.9)$ & $28.4(5.7)$ & $24.6(6.3)$ \\
\hline Mean BMI at booking (SD) & -------------- & $28.8(7.7)$ & $31.7(11.7)$ & $31.6(8.5)$ & $27.7(6.2)$ & $24.9(4.2)$ \\
\hline \multicolumn{7}{|l|}{ Parity $n(\%)$} \\
\hline Para 0 & $78(21.3)$ & \multirow[t]{3}{*}{ |----------- } & $1(1.3)$ & $9(11.5)$ & $45(57.7)$ & $23(29.5)$ \\
\hline Para 1-3 & $200(54.6)$ & & $14(7.0)$ & $51(25.5)$ & $108(54.0)$ & $27(13.5)$ \\
\hline$\geq$ para 4 & $87(23.7)$ & & $18(20.7)$ & $15(17.2)$ & $47(54.0)$ & $7(8.0)$ \\
\hline Missing & $1(0.3)$ & & & & & \\
\hline \multicolumn{7}{|l|}{ ANC status $n(\%)$} \\
\hline Registered & $221(60.4)$ & \multirow[t]{2}{*}{\begin{tabular}{|l|}
--------- \\
\end{tabular}} & $23(10.4)$ & $60(27.2)$ & $117(52.9)$ & $21(9.5)$ \\
\hline Not registered & $145(39.6)$ & & $10(6.9)$ & $16(11.0)$ & $83(57.2)$ & $36(24.8)$ \\
\hline
\end{tabular}




\begin{tabular}{|c|c|c|c|c|c|c|}
\hline $\begin{array}{l}\text { Gestational age at } A N C \\
\text { registration }\end{array}$ & & & & & & \\
\hline$\leq 12$ weeks & $12(3.3)$ & & $0(0)$ & $3(25.0)$ & $7(58.3)$ & $2(16.7)$ \\
\hline $13-20$ weeks & $69(18.9)$ & & $6(8.7)$ & $14(20.3)$ & $43(62.3)$ & $6(8.7)$ \\
\hline$>20$ weeks & $284(77.6)$ & & $27(9.5)$ & $58(20.4)$ & $150(52.8)$ & $49(17.3)$ \\
\hline Missing & $1(0.3)$ & & & & & \\
\hline $\begin{array}{l}\text { Gestational age at } \\
\text { diagnosis of } H D P^{*}\end{array}$ & & & & & & \\
\hline$<34$ weeks & $126(34.4)$ & & $23(18.3)$ & $11(8.7)$ & $77(61.1)$ & 15(11.9) \\
\hline$\geq 34$ weeks & $235(64.2)$ & & $10(4.3)$ & $61(26.0)$ & 122(51.9) & $42(17.9)$ \\
\hline Missing & $5(1.4)$ & & & & & \\
\hline $\begin{array}{l}\text { Moderate/severe } \mathrm{HTN}^{*} \\
\text { treatment before delive }\end{array}$ & & & & & & \\
\hline Yes & $123(33.4)$ & - & $29(23.6)$ & $27(22.0)$ & $61(49.6)$ & $6(4.9)$ \\
\hline No & $243(66.6)$ & & $4(1.6)$ & $49(20.2)$ & 139(57.2) & $51(21.0)$ \\
\hline Gestational age at deliv & & & & & & \\
\hline$<37$ weeks & $146(39.9)$ & & $15(10.3)$ & $8(5.5)$ & $97(66.4)$ & $26(17.8)$ \\
\hline$>=37$ weeks & $219(59.8)$ & & $18(8.2)$ & $67(30.6)$ & $103(47.0)$ & $31(14.2)$ \\
\hline Missing & $1(0.3)$ & & & & & \\
\hline Mode of delivery & & & & & & \\
\hline $\begin{array}{l}\text { Spontaneous vaginal } \\
\text { deliveries }\end{array}$ & $165(45.1)$ & & 18(10.9) & $42(25.5)$ & $82(49.7)$ & $23(13.9)$ \\
\hline $\begin{array}{l}\text { Assisted vaginal } \\
\text { deliveries }\end{array}$ & $6(1.6)$ & & $0(0)$ & $2(33.3)$ & $3(50.0)$ & $1(16.7)$ \\
\hline Caesarean section & $195(53.3)$ & & $15(7.7))$ & $31(15.9)$ & $115(59.0)$ & $34(17.4)$ \\
\hline Perinatal deaths $n(\%)$ & & & & & & \\
\hline Stillbirths & $29(65.9)$ & & $1(3.5)$ & $2(6.9)$ & $20(69.0)$ & $6(20.7)$ \\
\hline Early neonatal deaths & $12(27.3)$ & & $2(16.7)$ & $1(8.3)$ & $9(75.0)$ & $0(0)$ \\
\hline Late neonatal deaths & $3(6.8)$ & & $1(33.3)$ & $0(0)$ & $1(33.3)$ & $1(33.3)$ \\
\hline
\end{tabular}




\begin{tabular}{|c|c|c|c|c|c|c|}
\hline $\begin{array}{l}\text { Presence of peri-natal } \\
\text { complications other th } \\
\text { deaths }\end{array}$ & & & & & & \\
\hline Yes & $95(26.0)$ & \multirow[t]{2}{*}{---------- } & $9(9.5)$ & $12(12.6)$ & $61(64.2)$ & $13(13.7)$ \\
\hline No & $271(74.0)$ & & $24(8.9)$ & $63(23.3)$ & $139(51.5)$ & $45(16.3)$ \\
\hline \multicolumn{7}{|c|}{ Fetal weight at birth $(\mathrm{Kg})$} \\
\hline$<2.5$ & $140(38.3)$ & & $14(10.0)$ & $7(5.0)$ & $92(65.7)$ & $27(19.3)$ \\
\hline $2.5-4.0$ & $215(58.7)$ & & $16(7.4)$ & $66(30.7)$ & $103(47.9)$ & $30(14.0)$ \\
\hline$>4.0$ & $10(2.7)$ & & $3(30.0)$ & $2(20.0)$ & $5(50.0)$ & $0(0)$ \\
\hline missing & $1(0.3)$ & & & & & \\
\hline
\end{tabular}

${ }^{*} \mathrm{SD}=$ standard deviation, $\mathrm{ANC}=$ antenatal care, $\mathrm{HDP}=$ hypertensive disorders in pregnancy, $\mathrm{HTN}=$ hypertension, $\mathrm{CHT}=$ chronic hypertension, $\mathrm{GHT}$ = gestational hypertension, $\mathrm{PE}$ = pre-eclampsia

Table 2 shows the distribution of adherence in the postpartum period in HDP. Only about a third of all the women had their blood pressure measured between postpartum days 3 and 5 (i.e. $6.7 \%$, $16.4 \%, 59.7 \%$ and $17.2 \%$ for chronic hypertension, gestational hypertension, pre-eclampsia and eclampsia, respectively). Similarly, a third of women who had blood pressure above 140/90mmHg were not on antihypertensive medication within the first week of delivery (Figure 1).

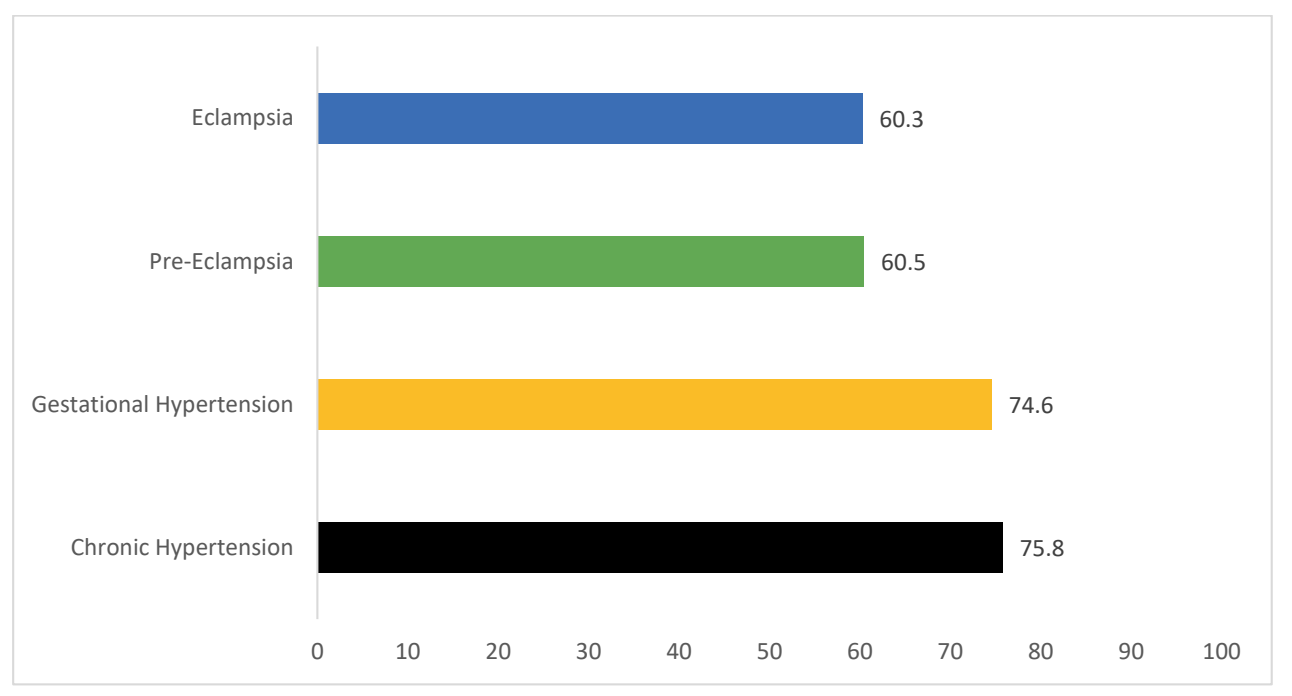

Figure 1: Proportion of women based on HDP sub-types with blood pressure above $140 / 90 \mathrm{mmHg}$ on antihypertensive medications within first week of delivery 
Only $63 \%$ and $58 \%$ were counseled on contraceptive services and the importance for early antenatal visit in future pregnancies, respectively. Fifty-four percent were not offered six to eight weeks postpartum medical review. The overall adherence to the recommended indicators for all women with $\mathrm{HDP}$ is $58 \%$ (i.e. $8.6 \%, 16 \%, 56.5 \%$ and $18.7 \%$ distributed as above, respectively).

Table 2: Proportion of women with any HDP who received recommended care and or counselling during puerperium (up to 8 weeks after delivery)

\begin{tabular}{|c|c|c|c|c|c|}
\hline Indicators & $\begin{array}{l}(\mathrm{N}=366) \\
\text { Number }(\%)\end{array}$ & $\begin{array}{l}\text { Chronic } \\
\text { Hypertension }\end{array}$ & $\begin{array}{l}\text { Gestational } \\
\text { Hypertension }\end{array}$ & Pre-eclampsia & Eclampsia \\
\hline $\begin{array}{l}\text { Received information on danger signs } \\
\text { (severe headache, blurring of vision, } \\
\text { nausea or vomiting, epigastric pain) in } \\
\text { the immediate postpartum period }\end{array}$ & $197(53.8)$ & $15(7.6)$ & $35(17.8)$ & $106(53.8)$ & $41(20.8)$ \\
\hline $\begin{array}{l}\text { Clinical wellbeing of babies assessed } \\
\text { daily in the first } 2-3 \text { days following } \\
\text { delivery? }\end{array}$ & $208(56.8)$ & $17(8.2)$ & $35(16.8)$ & $116(55.8)$ & $40(19.2)$ \\
\hline $\begin{array}{l}\text { Adequacy of babies' feeding assessed } \\
\text { daily in the first } 2-3 \text { days following } \\
\text { delivery }\end{array}$ & $209(57.1)$ & $17(8.1)$ & $36(17.2)$ & $118(56.5)$ & $38(18.2)$ \\
\hline $\begin{array}{l}\text { Blood pressure checked, at least, once } \\
\text { between day } 1 \text { and day } 2 \text { after } \\
\text { delivery }\end{array}$ & $360(98.4)$ & $32(8.9)$ & $73(20.3)$ & $199(55.3)$ & $56(15.6)$ \\
\hline $\begin{array}{l}\text { Blood pressure checked, at least, once } \\
\text { between day } 3 \text { and day } 5 \text { after } \\
\text { delivery }\end{array}$ & $134(36.6)$ & $9(6.7)$ & $22(16.4)$ & $80(59.7)$ & $23(17.2)$ \\
\hline $\begin{array}{l}\text { Women with blood pressure } \\
\text { measurement of } \geq 150 / 100 \mathrm{mmHg} \text { who } \\
\text { were on anti-hypertensive }\end{array}$ & $234(63.9)$ & $20(8.6)$ & $36(15.4)$ & $138(59.0)$ & $40(17.1)$ \\
\hline $\begin{array}{l}\text { Counselled on family planning at the } \\
6 \text {-weeks postnatal visit? Or at any } \\
\text { time before or after delivery }\end{array}$ & 231(63.1) & 21(9.1) & $45(19.5)$ & $120(52.0)$ & $45(19.5)$ \\
\hline $\begin{array}{l}\text { Counselled to attend ANC early in next } \\
\text { pregnancy }\end{array}$ & $213(58.2)$ & $20(9.4)$ & $30(14.1)$ & $121(56.8)$ & $42(19.7)$ \\
\hline $\begin{array}{l}\text { Patient counselled on risk of } \\
\text { recurrence of condition in subsequent } \\
\text { pregnancy (ies) }\end{array}$ & $198(54.1)$ & $20(10.1)$ & $21(10.6)$ & $117(59.1)$ & $40(20.2)$ \\
\hline $\begin{array}{l}\text { Babies received by a neonatologist at } \\
\text { delivery? }\end{array}$ & $202(55.2)$ & $18(8.9)$ & $34(16.8)$ & $118(58.4)$ & $32(15.8)$ \\
\hline $\begin{array}{l}\text { Offered a review between } 6-8 \text { weeks } \\
\text { postpartum }\end{array}$ & $168(45.9)$ & $16(9.5)$ & $22(13.1)$ & $93(55.4)$ & $37(22.0)$ \\
\hline
\end{tabular}


While about $83 \%$ of patients with PE/eclampsia had been offered urine protein estimation in the first week following delivery, only $42 \%$ had the same investigation between six to eight weeks postpartum. Ninety-three percent were not assessed for or administered thromboprophylaxis after delivery. Of those who remained hypertensive two weeks after discharge, only $24 \%$ were offered (specialist) medical review. None of the women with PE/eclampsia had their platelets, transaminases and serum creatinine measured after delivery. The overall adherence to the indicators was $44 \%$. Details of the distribution of adherence among women with pre-eclampsia and eclampsia in postpartum period are shown in Table $\mathbf{3}$.

Table 3: Proportion of women who had pre-eclampsia/eclampsia and offered recommended treatment and counselling at any given postpartum period

\begin{tabular}{|c|c|c|c|}
\hline \multirow[t]{2}{*}{ Indicators } & \multicolumn{3}{|l|}{ Number (\%) } \\
\hline & $\begin{array}{l}\text { Total } \\
258(100 \%)\end{array}$ & $\begin{array}{l}\text { Pre-eclampsia } \\
200(100 \%)\end{array}$ & $\begin{array}{l}\text { Eclampsia } \\
58(100 \%)\end{array}$ \\
\hline $\begin{array}{l}\text { Had PE/E and offered urine protein estimation within } 1 \text { week of } \\
\text { delivery }\end{array}$ & $214(82.9)$ & $162(81.0)$ & $52(89.7)$ \\
\hline $\begin{array}{l}\text { Had PE/E and offered urine protein estimation at } 6-8 \text { weeks } \\
\text { postpartum visits }\end{array}$ & $109(42.2)$ & $74(37.0)$ & $35(60.3)$ \\
\hline $\begin{array}{l}\text { Had PE/E and additional risk factor for thrombosis (e.g. Age }>35 \text {, } \\
\text { obesity and parity } \geq 3 \text {, etc.) received thromboprophylaxis at any } \\
\text { point after delivery }\end{array}$ & $19(7.4)$ & $13(6.5)$ & $6(10.3)$ \\
\hline $\begin{array}{l}\text { Had PE/E, planning for another pregnancy, counselled to maintain } \\
\text { health BMI before next pregnancy }\end{array}$ & $131(50.8)$ & $94(47.0)$ & $37(63.8)$ \\
\hline $\begin{array}{l}\text { Had PE/E, remained hypertensive } 2 \text { weeks after discharge, offered } \\
\text { medical review }\end{array}$ & $62(24.0)$ & $42(21.0)$ & 10(17.2) \\
\hline $\begin{array}{l}\text { Had PE/E, counseled to avoid a diuretic treatment if she is } \\
\text { breastfeeding her baby? }\end{array}$ & $154(49.6)$ & $92(46.0)$ & $36(62.1)$ \\
\hline $\begin{array}{l}\text { Had PE/E and Platelets, transaminases and serum creatinine } \\
\text { measured } 48 \text { to } 72 \text { hours after delivery }\end{array}$ & $0(0)$ & $0(0)$ & $0(0)$ \\
\hline Had PE/E received a dose of magnesium sulphate & 253(98.0) & $179(89.5)$ & $57(98.3)$ \\
\hline
\end{tabular}

Finally, Figure 2 shows trends in estimation and measurement of urine protein and blood pressure, respectively, within the first five days after delivery among women with all HDP subtypes. It shows a rapidly declining pattern of compliance and adherence from the day of delivery until the fifth postpartum day. For example, while $98 \%$ and $77 \%$ of the women, respectively, had their blood pressure and urine protein measured and documented on day 1 , 
only $37 \%$ and $24 \%$, respectively, had these measurements performed on day 5 postpartum whilst still on admission.

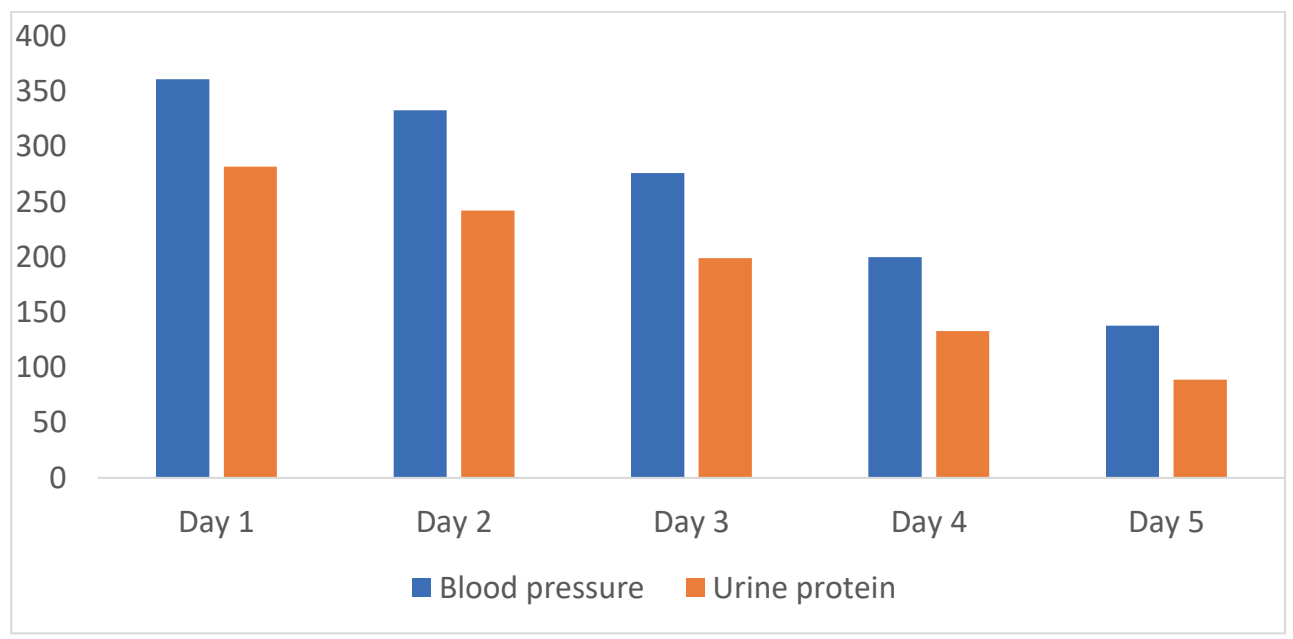

Figure 2: Proportion of women with HDP who had blood pressure and urine protein measured and documented between day $1-5$ after delivery

\section{Discussion}

In general, the level of adherence to the recommended postpartum care of the participants was poor. Overall, only $58 \%$ and $44 \%$ of all HDP and pre-eclampsia/eclampsia-specific indicators were met, respectively. For instance, it is important for women with HDP to have their BPs checked as at when due. This is because the values can rise unexpectedly to dangerously high levels especially between the third and fifth days postpartum. During this window period, two-thirds (64.4\%) of participants did not have their BPs checked, putting them at risk of adverse cardiovascular outcomes.

Two-thirds of women with HDP who had BP values above $140 / 90 \mathrm{mmHg}$ were not on antihypertensive medications. This is despite the widespread local availability of effective, lowcost drugs like nifedipine and $\alpha$-methyldop ${ }^{18}$. This represents an important missed opportunity to avert untoward clinical sequelae. While it is standard practice to review all women at six to eight weeks postpartum, over half of those with HDP in this study were not. This gave rise to another missed opportunity for smooth transition from obstetrics to specialist medical care in 
identifiable cases. These shortcomings could be adduced to ignorance as well as misleading perception of wellbeing in the women who are often free of significant symptoms during the period.

Pre-eclampsia is a recognized risk factor for development of VTE and guidelines recommend, at the very least, a risk assessment in the immediate postpartum period followed by administration of thrombo-prophylaxis if indicated. Almost all women with PE in this study, many of them with additional risk factors, were denied thrombo-prophylaxis after delivery. The near total lack of adherence to this recommendation suggests VTE is not considered a disease of priority in hospitals across the country. Some researchers have indeed put the annual prevalence of VTE in pregnancy and puerperium between 380 and 448 per 100,000 births ${ }^{19}$. The same study revealed that a quarter of wide spectrum of patients at risk of VTE in Africa do not receive prophylaxis. Contrary to guidelines, this study showed that only one-quarter of women with PE who remained hypertensive two weeks after delivery were offered (specialist) medical reviews. As a matter of fact, none of the participants had their platelets, transaminase and serum creatinine estimated within 48 - 72 hours after delivery. This is another substandard inpatient care factor that may have to do with the cost implications and inconveniences of such reviews and tests. Of importance is the fact that this study was conducted in tertiary hospitals with presumably better access to qualitative care and as such it is expected that levels of adherence to standard guidelines would likely be poorer at lower level facilities.

Although the evaluation of care offered to these women was mainly focused on the postpartum period, it was observed that a pattern of substandard care encompassed the entire maternity care continuum. Up to $40 \%$ of women did not receive any form of antenatal care (ANC), and only $5 \%$ registered in their first trimester. This is similar to the $37 \%$ ANC default rate reported by the most recent national demographic and health survey ${ }^{20}$. These findings are in sharp contrast to the WHO's vision that every pregnant woman receive quality care during pregnancy, childbirth and puerperium, including a first ANC visit in the first trimester ${ }^{21}$. This is especially relevant to women who might later develop HDP as ANC offers the opportunity for early detection and timely appropriate management, with substantial maternal and perinatal health benefits ${ }^{22-23}$. 


\section{Study limitations}

For this Nigerian study, indicators were seldom available as some postpartum services were not included, such as screening for causes of secondary hypertension in women with resistant hypertension, checking for resolution of biochemical changes in pre-eclampsia as well as mental health support. Evaluation of the latter would have added value as a component of comprehensive postpartum care. This would be required in this population to support grievances and bereavement processes as a consequence of adverse fetal or newborn outcomes ${ }^{24}$.

\section{Program and Policy implications}

By and large, our findings have significant program and policy implications in the global strive to reduce the burden of preventable maternal deaths in low- and middle-income countries. In order to achieve this, care for women with HDP should be looked upon as a continuum from preconceptual, pre-natal, intrapartum and through postpartum period ${ }^{19-21}$. This is especially in light of the very low early antenatal care attendance (first antenatal contact at $<12$ weeks of pregnancy) among our study participants. This observation is also relevant for international practice. While, overall, early antenatal care attendance has increased to 60\% globally in 2013 , pockets of inequality remain in LMICs with estimated less than $50 \%$ coverage $(25 \%$ for subSaharan Africa) as opposed to $85 \%$ in High Income Countries (HICs ${ }^{26}$. The current WHO guideline for the diagnosis and treatment of pre-eclampsia has re-emphasized the importance of early antenatal care attendance for all pregnant women ${ }^{13}$. However, there is a need to emphasize care of women with HDP beyond the immediate postpartum period. Hopefully, future reviews will correct this oversight.

\section{Conclusion}

Level of adherence to guidelines on postpartum management of HDP in tertiary hospitals in Nigeria is poor. Given the shortcomings observed, worse scenarios should be expected for secondary and primary level facilities. Due to the increased risk of morbidity and mortality associated with HDP in the postpartum period and beyond, it is recommended that institutionalization of these guidelines be prioritized and linked to the entire continuum of care 
beginning from adolescence, pregnancy, childbirth, postpartum to longer-term ongoing care by medical specialists, whenever indicated. 


\section{References}

1. Laura AM, Peter VD, William S, Mathew M. The FIGO textbook of pregnancy hypertension: An Evidence-based guide to monitoring, prevention and management. 2016; ISBN: 9780-9927545-5-6.

2. Noubiap JJ, Bigna JJ, Nyaga UF, et al. The burden of hypertensive disorders of pregnancy in Africa: A systematic review and meta-analysis. J Clin Hypertens. 2019; 21:479-488. https://doi.org/10.1111/jch.13514.

3. Oladapo OT, Adetoro OO, Ekele BA et al. Nigeria near-miss and Maternal Death Surveillance Network. When getting there is not enough: a nationwide cross-sectional study of 998 maternal deaths and 1451 near-misses in public tertiary hospitals in a lowincome country. BJOG 2015; DOI: 10.1111/1471-0528.13450.

4. Odum C, Akinkugbe A. The causes of maternal deaths in eclampsia in Lagos, Nigeria. West Afr J Med. 1991; 10:371-376.

5. Keskinkilic B, Engin-Ustun Y, Sanisoglu S et al. Maternal mortality due to hypertensive disorders of pregnancy, childbirth and puerperium between 2012 and 2015 in Turkey: a nation-based study. J Turk Ger Gynecol Assoc. 2017; 18:20-25. doi:10.4274/jtgga.2016.0244.

6. Nyfløt LT, EllingsenL, Yli BM, Øian P, Vangen S. Maternal deaths from hypertensive disorders: lessons learnt. Acta Obstet Gynecol Scand 2018; 97:976-987.

7. Deruelle $P$, Coudoux E, Ego A et al. Risk factors for post-partum complications occurring after preeclampsia and HELLP syndrome. A study in 453 consecutive pregnancies. Eur J Obstet Gynecol Reprod Biol 2006; 125:59-65.

8. Girsberger M, Muff C, Hösli I, Jan Dickenmann M. Short term sequelae of preeclampsia: a single center cohort study. BMC Pregnancy and Childbirth. 2018; 18:177. https://doi.org/10.1186/s12884-018-1796-z

9. Royal College of Obstetricians \& Gynaecologists. Green-top Guideline 37a: Reducing the risk of thrombosis and embolism during pregnancy and the puerperium. 2015; Available at: https://www.rcog.org.uk/en/guidelines-research-services/guidelines 
10. Melchiorre K, Sutherland G, Liberati M, Thilaganathan B. Preeclampsia is associated with persistent postpartum cardiovascular impairment. Hypertension 2011; 58:709-715.

11. Salomon A, Ishaku S, Kirk K, Warren C. Detecting and managing hypertensive disorders in pregnancy: a cross-sectional analysis of the quality of antenatal care in Nigeria. $B M C$ Health Services Research 2019; 19:411. doi.org/10.1186/s12913-019-4217-4218.

12. Ishaku S, Ahonsi B, Oginni A, Tukur J, Adoyi G. Obstetric knowledge of nurse educators in Nigeria: levels, regional differentials and their implications for maternal health delivery. Health Education Journal 1-10; 2015. DOI: 10.1177/0017896915571763.

13. Mark A. Brown, Laura A et al. Hypertensive Disorders of Pregnancy: ISSHP Classification, Diagnosis, and Management Recommendations for International Practice. Hypertension. 2018; 72:24-43. doi: 10.1161/HYPERTENSIONAHA.117.10803.

14. National Institute for Health and Care Excellence (NICE) guideline. Hypertension in pregnancy: diagnosis and management. 2019. www.nice.org.uk/guidance/ng133

15. WHO recommendations for Prevention and treatment of pre-eclampsia and eclampsia. World Health Organization, 20 Avenue Appia, 1211 Geneva 27, Switzerland. ISBN 97892 41548335.

16. Population Council, Nigerian Federal Ministry of Health. National training manual on treatment of pre-eclampsia and eclampsia for doctors, nurses and midwives. Population Council. Abuja. 2017.

17. Sharif H, Kanij S, Salma R et al. Hypertensive disorders in pregnancy: assessing postnatal quality of care and outcomes for women and their infants in Bangladesh. (c) 2019 The Population Council, Inc.

18. Oke O, Adedapo A. Antihypertensive Drug Utilization and Blood Pressure Control in a Nigerian Hypertensive Population. General Med 3: 1000169. 2015; doi:10.4172/23275146.1000169.

19. Danwang C, Temgoua M, Agbor V, Tankeu A, Noubiap J. Epidemiology of venous thromboembolism in Africa: a systematic review. J Thromb Haemost 2017; https://doi.org/10.1111/jth.13769 
20. National Population Commission (NPC) [Nigeria] and ICF. Nigeria Demographic and Health Survey 2018. Abuja, Nigeria, and Rockville, Maryland, USA: NPC and ICF. 2019.

21. World Health Organization. Recommendations on antenatal care for a positive pregnancy experience. ISBN 9789241549912.

22. von DP, Magee LA. Preventing deaths due to the hypertensive disorders of pregnancy. Best Practice \& Research Clinical Obstetrics and Gynecology 2016;36 83e102.

23. Moore N, Blouin B, Razuri H, Casapia M, Gyorkos TW. Determinants of first trimester attendance at antenatal care clinics in the Amazon region of Peru: A case-control study. 2017. PLOS ONE 12: e0171136.doi: 10.1371/journal.pone.0171136.

24. Simhi M, Sarid O, Cwikel J. Preferences for mental health treatment for post-partum depression among new mothers. Isr J Health Policy Res 8, 84 (2019). https://doi.org/10.1186/s13584-019-0354-0

25. Kavita S, William TS, Allisyn CM. Assessing the Continuum of Care Pathway for Maternal Health in South Asia and Sub-Saharan Africa. Matern Child Health J. 2016; 20: 281-289. doi:10.1007/s10995-015-1827-6.

26. Iqbal S, Maqsood S, Zakar, R. et al. Continuum of care in maternal, newborn and child health in Pakistan: analysis of trends and determinants from 2006 to 2012. BMC Health Serv Res 17, 189. 2017. https://doi.org/10.1186/s12913-017-2111-9

27. Sakuma S, Yasuoka J, Phongluxa K, Jimba M. Determinants of continuum of care for maternal, newborn and child health services in rural Khammouane, Lao PDR. PLOS ONE (2019) 14: e0215635.https://doi.org/10.1371/journal.pone.0215635.

28. Ann-Beth M, Max P, Doris C, Lale S. Early antenatal care visit: a systematic analysis of regional and global levels and trends of coverage from 1990 to 2013. Lancet Glob Health 2017; 5: e977-83. 



\section{Part II}

Chronic medical disorders associated with prior hypertensive disorders in pregnancy 



\section{Chapter 4}

Persistent hypertension up to one year postpartum among women with hypertensive disorders in pregnancy in a low-resource Setting: A prospective cohort study

\section{Salisu M Ishaku}

Tukur Jamilu

Agbo Innocent

Gbenga A Kayode

Dattijo M Lamaran

Oyeneyin Lawal

Warren E Charlotte

Owa 0 Olorunfemi

Hannifa D Abubakar

Tunau Karima

Odusolu O Patience

Abdulkarim Musa

Onyebuchi K Azubuike

Aminu M Baffah

Arie Franx

Diederick E Grobbee

Joyce L Browne

Submitted to Global Heart Journal for publication, responded to reviewers' comments and awaiting decision 


\begin{abstract}
Background

Hypertensive disorders in pregnancy (HDP) are associated with lifelong increased cardiovascular disease risk. Persistent hypertension in women with HDP during the postpartum period could suggest progression to the development of chronic hypertension. Outcomes of postpartum follow up of women with HDP in low- and middle-income countries (LIMCs) have not been well examined, and most previous follow-ups typically last for maximally six weeks postpartum. We assessed the prevalence of persistent hypertension up to one year in women with HDP in a low resource setting and determined associated risk factors.
\end{abstract}

\title{
Methodology
}

A prospective cohort study was conducted at eight tertiary health care facilities in seven states of Nigeria. Four hundred and ten women with any HDP were enrolled within 24 hours of delivery and followed up at nine weeks, six months and 1 year postpartum. Descriptive statistics were performed to express the participants' characteristics. Univariable and multivariable logistic regressions were conducted to identify associated risk factors.

\section{Results}

Of the 410 women enrolled, 278 were followed up to 1 year after delivery (follow up rate 68\%). Among women diagnosed with gestational hypertension and pre-eclampsia/eclampsia, $22.3 \%$ $(95 \% \mathrm{Cl} ; 8.3-36.3)$ and $62.1 \%(95 \% \mathrm{Cl} ; 52.5-71.9)$, respectively, had persistent hypertension at 6 months and this remained similar at one year $22.3 \%(95 \% \mathrm{Cl} ; 5.6-54.4)$ and $61.2 \%(95 \% \mathrm{Cl}$; 40.6 - 77.8), respectively). Maternal age and body mass index were significant risk factors for persistent hypertension at one year after delivery [aORs $=1.07 /$ year $(95 \% \mathrm{Cl} ; 1.02-1.13)$ and $1.06 / \mathrm{kg} / \mathrm{m}^{2}$ (95\% Cl; 1.01-1.10)], respectively. 


\section{Conclusion}

This study showed a substantial prevalence of persistent hypertension beyond puerperium. Health systems in LMICs need to be organized to anticipate and maintain postpartum monitoring until blood pressure is normalized, or women are referred for further medical review or discharged to family physicians as appropriate. In particular, attention should be given to women who are obese, and or of higher maternal age both during postpartum and pre-conception periods.

Keywords: Hypertensive Disorder in Pregnancy, Persistent Hypertension, Nigeria 


\section{Introduction}

Hypertensive disorders in pregnancy (HDP) place a huge burden on maternal and child health with at least 70,000 and 500,000 maternal and neonatal deaths, respectively, annually ${ }^{1,2}$. Globally HDP complicates $5-10 \%$ of pregnancies ${ }^{1,2}$. The spectrum includes chronic hypertension (secondary or essential), gestational hypertension and (pre-)eclampsia ${ }^{2}$. In Nigeria, pre-eclampsia contributes to over $23 \%$ of the direct causes of maternal mortality in referral health care facilities ${ }^{3}$. Bearing in mind delivery of the placenta is considered the cure for HDP (except chronic hypertension), the traditional expectation is that hypertension resolves by the end of the puerperium, a period generally defined as six to eight weeks after delivery ${ }^{4}$. However, emerging evidence suggests that hypertension may persist for a variable period beyond puerperium and up to years after delivery - especially following severe forms of HDP such as pre-eclampsia and eclampsia ${ }^{5,6}$. This is due to combination of two factors. First, women with HDP have an increased lifetime risk of future chronic hypertension, cardiovascular disease risk factors and cardiovascular diseases due to underlying vascular (re)consistitution or HDP-related (vascular) damage 7-8 $^{7}$. Second, women with previous undiagnosed chronic hypertension are misclassified during pregnancy as gestational hypertension, ${ }^{7,34-35}$ and their persisting hypertension beyond puerperium erroneously considered new onset chronic hypertension following HDP.

Therefore, estimation and quantification of the true magnitude of persistent hypertension in HDP is an important initial step to curb the risk of future chronic hypertension and adverse cardiovascular events ${ }^{7-8}$. While a rising trend in premature mortality from CVDs is evident in LMICs, sub-Saharan Africa suffers disproportionately ${ }^{9}$. Despite these facts, few studies have been conducted in low-resource settings and the available evidence is usually restricted to short-term observation between six weeks and six months after delivery ${ }^{10-14}$. This study prospectively determined the prevalence of hypertension over one year in a cohort of Nigeria's women with HDP who delivered in tertiary facilities and assessed the risk factors associated with persistent hypertension. 


\section{Methods}

Study design

The study was a prospective cohort study. Women with HDP who delivered in the participating health facilities were recruited from August 2017 to April 2018 and followed up over a subsequent period of one year. The last woman recruited exited the study on March 31, 2019 after one year of follow up.

\section{Study setting}

The study was conducted at eight tertiary hospitals in the six geo-political zones of Nigeria. The hospitals were purposefully selected to reflect diversity in the country in terms of ethnic differences and socio-economic status. The following states (and hospitals) participated: Bauchi State (Abubakar Tafawa Balewa University Teaching Hospital, ATBUTH), Cross River State (University of Calabar Teaching Hospital, UCTH), Ebonyi State (Federal Teaching Hospital Abakaliki, FTHA), Kogi State (Federal Medical Center, FMC Lokoja), Kano State (Aminu Kano Teaching Hospital, AKTH), Ondo State (Mother and Child Hospital Akure and University of Medical Sciences Teaching Hospital, Ondo) and Sokoto State (Usmanu Danfodio University Teaching Hospital, UDUS). See figure I below depicting study locations:

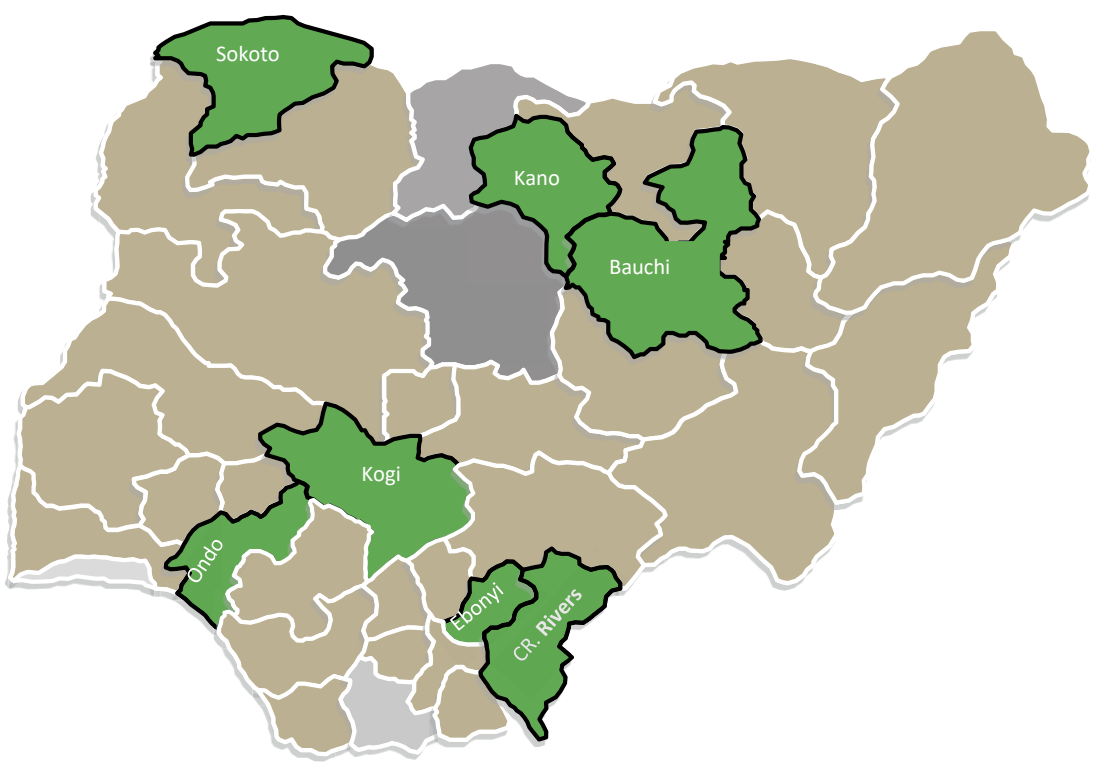


Because of the geographical spread of the health facilities, women who received care in these facilities were considered a good representation of the diversity in the country. The health facilities, being tertiary, were high-volume sites with well-functioning ante- and postnatal clinics, delivery rooms, and laboratory services with combined annual deliveries averaging 38,400.

\section{Participants}

Women who delivered at the facilities (regardless of where they received their antenatal care), who were 18 years or above, and had a diagnosis of HDP were eligible for inclusion. Women with the following conditions were excluded: multiple pregnancies, medical disorders in pregnancy other than HDP (e.g., diabetes mellitus, sickle cell disease, heart disease, kidney and other connective tissues disorders).

\section{Study procedures}

Women were informed of the study either during antenatal care period (for registered clients) or after delivery but all recruitments were done in the postpartum period. Those willing to participate were individually counseled, informed consent obtained - either signed or thumb printed- (consent rate over 95\%) and enrollment forms completed within 24 hours of delivery. The enrollment form was a questionnaire that collected information on socio-demographic and obstetrics variables such as age, body mass index (BMI), parity and booking status. After enrollment, the women underwent baseline clinical evaluations to assess their symptoms and signs. The clinical evaluation involved general and systemic examination including the determination of the blood pressure measurements. In addition, laboratory investigations were performed on the participants before they were discharged from the hospitals. They were subsequently followed-up during which the same clinical and laboratory investigations conducted at baseline were repeated at nine weeks, six months and one year postpartum. To improve follow up rate over the entire duration of one year, the research participants were requested (if willing and consented) to provide their contact information (particularly personal and spousal mobile telephone numbers). They were reminded of their follow up appointments through phone calls. Participants' contact information was not linked to their clinical records while all clinical information was linked to unique identifiers. 


\section{Exposures variables}

The main exposure of interest was the presence of any of the HDP sub-types including chronic hypertension, gestational hypertension and pre-eclampsia as defined by the International Society for the Study of Hypertension in Pregnancy (ISSHP) ${ }^{1,2}$.

Hypertension was defined as systolic blood pressure of $\geq 140 \mathrm{mmHg}$ and/or diastolic blood pressure of $\geq 90 \mathrm{mmHg}$ measured on two consecutive periods 4-6 hours apart. Chronic hypertension in pregnancy was defined as any hypertension with onset before the index pregnancy or diagnosed within the first 20 weeks of the index pregnancy. Gestational hypertension was defined as any hypertension occurring after the first 20 weeks of pregnancy without significant proteinuria (<2++ of proteinuria on urine dipstick measurement) or any hematological or biochemical abnormality. Pre-eclampsia was defined as hypertension with onset after the first 20 weeks of pregnancy with significant proteinuria ( $\geq 2++$ of proteinuria on urine dipstick measurement) or the presence of any hematological and biochemical abnormality.

\section{Outcome variables}

The main outcome variable was persistent hypertension, defined as the occurrence of systolic blood pressure of $\geq 140 \mathrm{mmHg}$ and/or, diastolic blood pressure of $\geq 90 \mathrm{mmHg}$ measured at least 46 hours apart after 8 weeks postpartum. Other outcome measures of interest included persistent significant proteinuria after delivery ( $\geq 2++$ of proteinuria on urine dipstick measurement), BMIs (weight $(\mathrm{kg}) /$ height $\left(\mathrm{m}^{2}\right)$ ), abnormally high serum creatinine greater than $90 \mathrm{mmol} / \mathrm{L}$ (range $=45-$ $90 \mathrm{mmol} / \mathrm{L}$ ), abnormally high serum urea greater than $7.1 \mathrm{mmol} / \mathrm{L}$ (range $=2.5-7.1 \mathrm{mmol} / \mathrm{L}$ ), abnormally high uric acid greater than $446 \mathrm{mmol} / \mathrm{L}$ (range $209-446 \mathrm{mmol} / \mathrm{l}$ ), raised Alanine transaminase (ALT) above 55 units/liter (range $=7-55$ ), raised Aspartate transaminase (AST) above 48 units per/liter (range $=8-48$ ), thrombocytopenia (below 150, 000 counts per microliter, range $=150,000-450,000$ ), hypercholesterolemia above $200 \mathrm{mg} / \mathrm{dl}$ and hyper triglycerides above 1.7 millimoles per liter. 


\section{Data source/data collection}

At each data collection period (baseline: i.e., within 24 hours of delivery, nine weeks, six months and one year postpartum), we measured participants' BMIs (weight $(\mathrm{kg}) /$ height $\left(\mathrm{m}^{2}\right)$ ), blood pressure (by auscultation technique using mercury sphygmomanometer, first and fourth Korortkoff sounds were taken as systolic and diastolic values, respectively), urine protein (using meditest combi 10 dipsticks) and a general physical examination for symptoms and signs conducted. In addition, blood samples were collected for laboratory tests which included blood platelet estimation, liver function tests (AST, ALT), renal function assessment (serum urea, creatinine and uric acids), glucose tolerance test (random and fasting blood sugar) and serum lipids (cholesterols and triglycerides). All clinical examinations and blood sample collection were performed by trained medical officers who served as research assistants for this study. Laboratory investigations were performed by laboratory analists based on universal laid down protocol and standard practices used in all public tertiary hospitals in Nigeria (supplement 1).

\section{Risk of bias}

In order to reduce biases, participants were recruited from a similar population of women giving birth in these facilities. Since in Nigeria women go to tertiary hospitals for antenatal and delivery services based on their places of residence and socio-economic status (not based on referrals), a possible selection bias in the direction of high risk confers by socio-economic status cannot be rule-out. Recruitment proceeded independently and concurrently in all facilities until the desired sample size was reached. Case identification was done by specially trained and experienced midwives using standard diagnostic criteria. Outcomes assessors were not aware of clients' categorization into various HDP categories. The results of medical and laboratory investigations were entered in the electronic data capturing platform by trained research assistants as soon as the data were available to reduce the incidence of missing data. The hard copies of all medical and laboratory results were retained as source documents at the facilities for future reference when necessary. 


\section{Data analysis plan/statistical methods}

Frequencies, percentages and simple proportion (mean and standard deviations) were used to describe participants' obstetrics, demographics, and other variables by HDP group. Women with eclampsia were included in the pre-eclampsia sub-classification, and women with pre-existing hypertension (chronic hypertension) were not analyzed beyond their proportion in the study population. We used bar charts to illustrate trends in magnitudes of blood pressure response since delivery up to one year thereafter. Trends to blood pressure normalization since delivery were analyzed, using proportions and percentages, separately for chronic hypertension, gestational hypertension and pre-eclampsia. Univariable and multivariable logistic regression analysis was used to determine factors that predict the presence or absence of hypertension at one year after delivery. Possible predictor variables were hypothesis-driven selected based on previous literature and clinical knowledge. The data were analyzed using SPSS IBM version 25.0.

\section{Missing data}

During every follow-up period, clinical and laboratory information were obtained and entered immediately into the electronic data capturing platform to minimize instances of missing data. Cases of missing data occurred when subjects failed to report for data collection periods. We assume that data were missing completely at random. Therefore, complete case analysis was performed, such that for any data collection period, only clients that have reported and provided complete information were analyzed.

\section{Ethical approval}

The study was approved by the Population Council's institutional review board in New York (protocol no. 810), National Health Research Ethics Committee (NHREC) at the Federal Ministry of Health and by the institutional review boards at all the participating hospitals.

\section{Results}

The recruitment and follow up of study participants are depicted in figure 2 . Of the 410 women with HDP who were enrolled, 407 provided baseline data and 263, 232 and 278 women followed up at nine weeks, six months and one year, respectively (a $68 \%$ follow up rate at one year). 
Figure 2: Flowchart of Participants enrollment and follow up process*

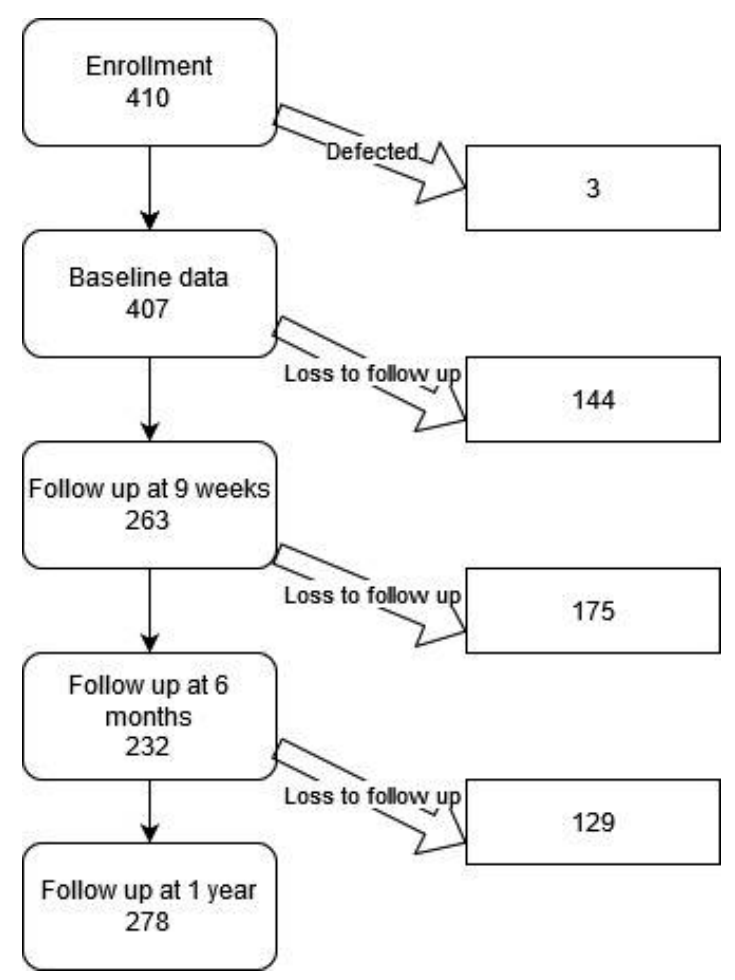

Table 1 presents the baseline characteristics of the participants. The majority of women $(60 \%, n=$ 247) had antenatal care, $3 \%$ of whom registered in the first trimester. The mean gestational ages at the onset of HDP and delivery were 33 weeks (standard deviation (sd) 8.8) and 36 (sd 4.1) weeks, respectively. The mean BMI at booking among those who received antenatal care was $28 \mathrm{~kg} / \mathrm{m}^{2}$ (sd 7.7). The perinatal death rate was 9\% (35): $7 \%$ (29) and $2 \%(6)$ for stillbirths and early neonatal deaths respectively. 
Table 1: Socio-demographic and obstetric characteristics of the of 410 women with HDPs enrolled in the study

\begin{tabular}{|c|c|c|c|c|c|c|}
\hline Variables & Number (\%) & Mean (SD) & GH & CHT & PE & EC \\
\hline Mean age (SD) & & $28.9(6.4)$ & $33.3(28.9)$ & $35.2(6.8)$ & $28.4(5.7)$ & $24.6(6.3)$ \\
\hline Mean BMI at booking (SD) & & $28.8(7.7)$ & $31.6(8.5)$ & $31.7(11.7)$ & $27.7(6.2)$ & $24.9(4.2)$ \\
\hline \multicolumn{7}{|l|}{ Parity (N=366) } \\
\hline Para 0 n (\%) & $78(21.4)$ & & $9(12.0)$ & $1(3.0)$ & $45(22.5)$ & $23(40.3)$ \\
\hline Para $1-3 n(\%)$ & $200(54.8)$ & & $51(68.0)$ & $14(42.4)$ & $108(54)$ & $27(47.4)$ \\
\hline zpara 4 n (\%) & $87(23.8)$ & & $15(20.0)$ & $18(54.6))$ & $47(23.5)$ & $7(12.3)$ \\
\hline \multicolumn{7}{|l|}{ Booking status $n(\%)$} \\
\hline Booked & $247(60.2)$ & & $60(80.0)$ & $23(70.0)$ & $117(58.5)$ & $21(36.8)$ \\
\hline Unbooked & $163(39.8)$ & & $15(20.0)$ & $10(30.0)$ & $83(41.5)$ & $36(63.2)$ \\
\hline Mean Gestational age at booking & & & $24.9(6.7)$ & $23.8(4.6)$ & $23.1(6.3)$ & $23(6.7)$ \\
\hline \multicolumn{7}{|l|}{ Distribution of GA at booking $n(\%)$} \\
\hline$\leq 12$ weeks & $14(3.4)$ & & $3(4.0)$ & $0(0.0)$ & $7(3.5)$ & $2(3.5)$ \\
\hline $13-20$ weeks & $73(17.8)$ & & $14(18.7)$ & $6(18.2)$ & $43(21.5)$ & $6(10.5)$ \\
\hline$>20$ weeks & $323(78.8)$ & & $58(77.3)$ & $27(81.8)$ & $150(75)$ & $49(86)$ \\
\hline Mean Gestational age at onset of HDP & & $33.2(8.8)$ & $36.9(4.1)$ & ------------- & $33(8.5)$ & $34.5(6.5)$ \\
\hline \multicolumn{7}{|l|}{ GA distribution of onset of HDP } \\
\hline$\geq 34$ weeks & $239(65.5)$ & & $61(81.3)$ & --------- & $122(61)$ & $42(73.7)$ \\
\hline$<34$ weeks & $126(34.5)$ & & $11(14.7)$ & --------- & $78(39)$ & $15(26.3)$ \\
\hline HDP sub-types & & & & & & \\
\hline
\end{tabular}




\begin{tabular}{|c|c|c|c|c|c|c|}
\hline Variables & Number (\%) & Mean (SD) & GH & CHT & PE & EC \\
\hline Chronic hypertension & $33(9.0)$ & ----------- & --------- & |-------- & |--------- & ---------- \\
\hline Gestational hypertension & $75(20.5)$ & ------------ & --------- & --------- & ----------' & ----------' \\
\hline Pre-eclampsia & $200(54.5)$ & --------------- & -------- & --------' & 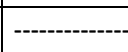 & - \\
\hline Eclampsia & $58(16.0)$ & & & & & \\
\hline Mean Gestational age at delivery (SD) & & $36.5(4.1)$ & $38.7(1.9)$ & $36.1(3.9)$ & $36(4.3)$ & $35.5(4.3)$ \\
\hline \multicolumn{7}{|l|}{ Distribution of GA at delivery $n$ (\%) } \\
\hline$>=37$ weeks & $265(64.6)$ & & $67(89.3)$ & $18(54.5)$ & $103(51.5)$ & $31(54.4)$ \\
\hline$<37$ weeks & $145(35.4)$ & & $8(10.7)$ & $15(45.5)$ & $97(48.5)$ & $26(45.6)$ \\
\hline \multicolumn{7}{|l|}{ Mode of Delivery $n$ (\%) } \\
\hline Spontaneous Vaginal Delivery & $165(45.1)$ & ------------- & $42(56)$ & $18(54.5)$ & $82(41)$ & $23(40.4)$ \\
\hline Assisted Vaginal Delivery & $6(1.6)$ & 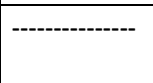 & $2(2.7)$ & $0(0.0)$ & $3(1.5)$ & $1(1.8)$ \\
\hline Elective Caesarean section & $19(5.2)$ & 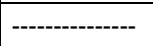 & $6(8)$ & $2(6.1)$ & $11(5.5)$ & $0(0.0)$ \\
\hline Emergency Caesarean section & $176(48.1)$ & -------------- & $25(33.3)$ & $13(39.4)$ & $104(52)$ & $33(57.8)$ \\
\hline \multicolumn{7}{|l|}{ Apgar score at $1 \mathrm{~min} n(\%)$} \\
\hline Apgar $>=7$ & $224(61.4)$ & & $58(77.3)$ & $20(60.6)$ & $117(58.5)$ & $29(50.9)$ \\
\hline Apgar $<7$ & $141(38.6)$ & & $17(22.7)$ & $13(39.4)$ & $83(41.5)$ & $28(49.1)$ \\
\hline \multicolumn{7}{|l|}{ Apgar score at $5 \min n(\%)$} \\
\hline Apgar $>=7$ & $308(84.4)$ & & $72(96.0)$ & $29(87.9)$ & $163(81.5)$ & $44(77.2)$ \\
\hline Apgar $<7$ & $57(16.6)$ & & $3(4.0)$ & $4(12.1)$ & $37(18.5)$ & $13(22.8)$ \\
\hline
\end{tabular}




\begin{tabular}{|c|c|c|c|c|c|c|}
\hline Variables & Number (\%) & Mean (SD) & GH & CHT & $\mathrm{PE}$ & EC \\
\hline \multicolumn{7}{|l|}{ Perinatal deaths $n(\%)$} \\
\hline Stillbirths & $29(70.7)$ & ---------------- & $2(66.7)$ & $1(33.3)$ & 20(69) & $6(100.0)$ \\
\hline Early neonatal deaths & $12(29.3)$ & --------------- & $1(33.3)$ & $2(66.7)$ & 9(31) & $0(0.0)$ \\
\hline $\begin{array}{l}\text { Proportion of HDPs with BP } \\
\geq 150 / 100 \mathrm{mmHg} \text { within } 5 \text { days of } \\
\text { delivery on anti-hypertensive } \\
\text { medications }\end{array}$ & $33 \%$ & ------------- & & & & \\
\hline
\end{tabular}

$\mathrm{HDP}=$ Hypertensive disorders in pregnancy, $\mathrm{SD}=$ standard deviation, $\mathrm{GHT}=$ Gestational hypertension, $\mathrm{CHT}=$ Chronic hypertension, $\mathrm{PE}=\mathrm{Pre}-$ eclampsia, $\mathrm{EC}=$ Eclampsia, $\mathrm{GA}=$ gestational age, $\mathrm{BP}=$ Blood pressure

The proportion of women with persistent hypertension (systolic of $\geq 140 \mathrm{mmHg}$ and or diastolic of $\geq 90 \mathrm{mmHg}$ ) at nine weeks, six months and one year after delivery is shown in Figure 3 . For women with gestational hypertension, persistent hypertension at nine weeks, six months and one year occurred in $23 \%(95 \% \mathrm{Cl} ; 6.6-52.6), 22 \%$ (95\% Cl; $8.3-36.3)$ and 22\%, (95\% Cl; $5.6-$ 54.4) respectively. Women with pre-eclampsia/eclampsia had a much higher prevalence: $62 \%$ (95\% Cl; $47.9-76), 62 \%(95 \% \mathrm{Cl} ; 52.5$ - 71.9) and 61\% (95\% Cl; $40.6-77.8)$, respectively. 
Figure 3: Prevalence of persistent hypertension $(B P>140 / 90 \mathrm{mmHg}$ ) based on HDP sub-types and total over one year of follow up

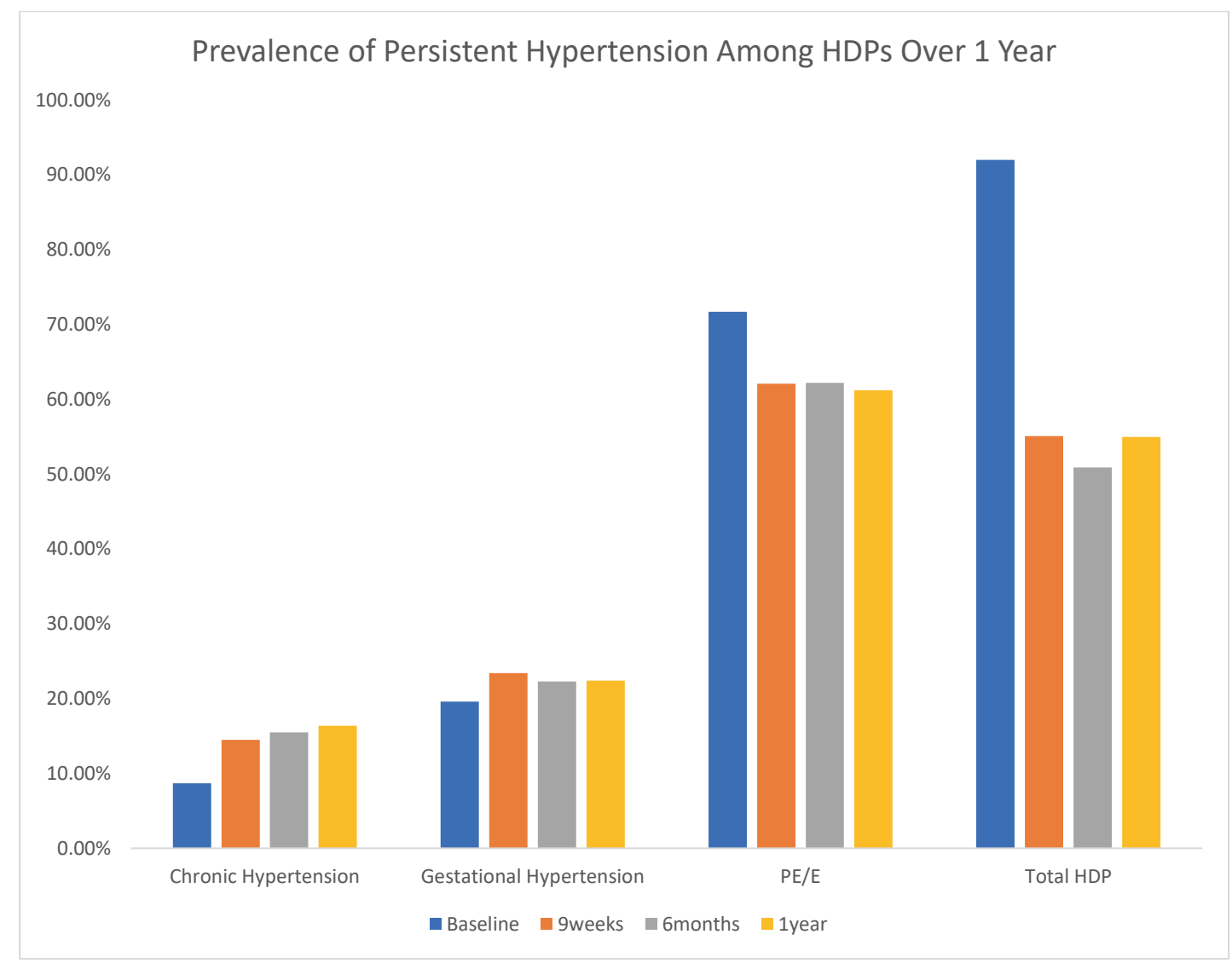

HDPs = Hypertensive disorders in pregnancy; $\mathrm{PE} / \mathrm{E}=$ Pre-eclampsia/eclampsia

The mean systolic and diastolic blood pressure (Figure 4) declined progressively from delivery baseline values of $160 \mathrm{mmHg}$ and $102.5 \mathrm{mmHg}$ respectively to a one-year mean values of 132.9 $\mathrm{mmHg}$ and $86.9 \mathrm{mmHg}$, respectively. Within the first five postpartum days, only $33 \%$ of those with high blood pressure were on anti-hypertensive therapy. 
Figure 4: Trend in mean systolic and diastolic blood pressure over one year of follow up:

\section{Mean BP of HDP client over time}

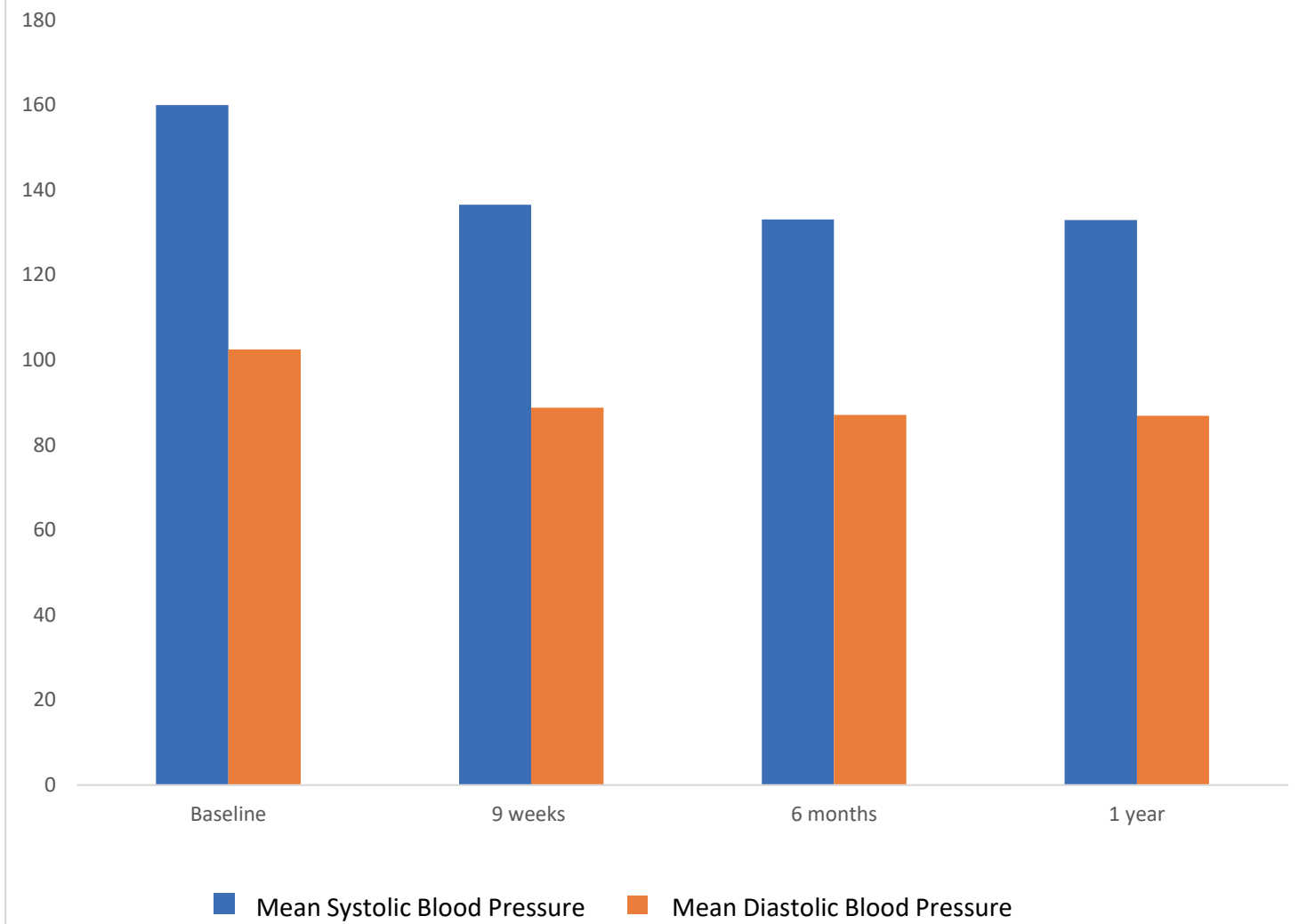

\footnotetext{
$\mathrm{BP}=$ Blood pressure, $\mathrm{HDP}=$ Hypertensive disorders in pregnancy
}

Table 2 shows the results of univariable and multivariable logistic regression to assess the association between 13 possible risk factors for the presence of persistent hypertension one year after delivery among women with gestational hypertension and pre-eclampsia and eclampsia(chronic hypertension in pregnancy excluded in the determinants). Of these predictors, maternal age, BMI, systolic and diastolic blood pressure at delivery significantly predicted the presence of persistent hypertension at one year in univariable analysis [(ORs $=1.11 /$ year $(95 \% \mathrm{Cl}$; $1.06-1.15), 1.03 / \mathrm{kg} / \mathrm{m}^{2}$ (95\% Cl; $\left.1.00-1.07\right), 1.01 / \mathrm{mmHg}(95 \% \mathrm{Cl} ; 1.00-1.02)$ and 1.02/mmHg 
(95\% Cl; $1.00-1.03)]$ respectively]. In the multivariable analysis, only maternal age and BMI at delivery were independent predictors and increased the risk of persistent hypertension at one year after delivery following HDP [adjusted OR (1.07/year $(95 \% \mathrm{Cl} ; 1.02-1.13)$ and $1.06 / \mathrm{kg} / \mathrm{m}^{2}$ (95\% Cl; 1.01-1.1)] respectively.

Table 2: Univariate and multivariable logistic regression analysis of factors associated with persistent hypertension among $\mathbf{2 5 4}$ women with HDPs (excluding chronic hypertension) at one year after delivery

\begin{tabular}{|c|c|c|c|c|}
\hline \multirow{2}{*}{ Variable } & \multicolumn{2}{|l|}{ Univariate } & \multicolumn{2}{|l|}{ Multivariate } \\
\hline & OR (95\% Cl) & P-value & OR $(95 \% \mathrm{Cl})$ & P-value \\
\hline Age & $1.11(1.06-1.15)$ & 0.000 & $1.07(1.02-1.13)$ & 0.011 \\
\hline $\mathrm{BMI}$ at baseline & $1.03(1.00-1.07)$ & 0.147 & $1.06(1.01-1.10)$ & 0.010 \\
\hline $\begin{array}{l}\text { Random blood glucose at } \\
\text { baseline }\end{array}$ & $0.99(0.90-1.09)$ & 0.869 & $0.92(0.80-1.06)$ & 0.258 \\
\hline $\begin{array}{l}\text { Gestational age at onset } \\
\text { of HDP }\end{array}$ & $0.97(0.94-1.00)$ & 0.055 & $0.99(0.96-1.05)$ & 0.948 \\
\hline Gestational age at delivery & $0.97(0.92-1.04)$ & 0.513 & $0.98(0.91-1.07)$ & 0.729 \\
\hline Systolic BP at baseline & $1.01(1.00-1.02)$ & 0.030 & $1.01(0.99-1.03)$ & 0.633 \\
\hline Diastolic BP at baseline & $1.02(1.00-1.03)$ & 0.067 & $1.01(0.98-1.04)$ & 0.421 \\
\hline $\begin{array}{l}\text { Diagnosis of gestational } \\
\text { hypertension }\end{array}$ & $0.25(0.09-0.71)$ & 0.009 & $0.71(0.44-1.15)$ & 0.164 \\
\hline $\begin{array}{l}\text { Diagnosis of } \\
\text { eclampsia }\end{array}$ & $0.20(0.08-0.52)$ & 0.001 & & \\
\hline $\begin{array}{lll}\text { Raised } & \text { creatinine } & >90 \\
\mathrm{mmol} / \mathrm{L} & & \\
\end{array}$ & $1.04(0.90-1.20)$ & 0.061 & $1.06(0.90-1.25)$ & 0.477 \\
\hline $\begin{array}{l}\text { Raised uric Acid } \\
>446 \mathrm{mmol} / \mathrm{L}\end{array}$ & $1.01(0.95-1.06)$ & 0.797 & $1.03(0.96-1.10)$ & 0.471 \\
\hline $\begin{array}{l}\text { Raised } \\
\text { Triglycerides }>1.7 \mathrm{mmol} / \mathrm{l}\end{array}$ & $0.99(0.88-1.11)$ & 0.871 & $0.95(0.80-1.12)$ & 0.520 \\
\hline $\begin{array}{l}\text { Raised } \\
\text { cholesterol }>200 \mathrm{mg} / \mathrm{dL}\end{array}$ & $0.99(0.99-1.00)$ & 0.634 & $0.99(0.99-1.00)$ & 0.594 \\
\hline
\end{tabular}

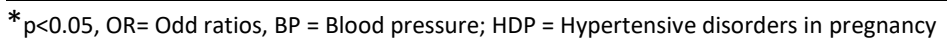




\section{Discussion}

To date this cohort study of women with HDP recruited and followed up from delivery until one year afterward is the largest and longest conducted in a sub-Saharan African setting. This study observed high rates of persistent hypertension regardless of HDP subtype ( $21 \%$ and $61 \%$ of women with gestational hypertension and pre-eclampsia, respectively). This is in line with findings from other studies within sub-Sahara Africa in Nigeria, Cameroon, Uganda and Sudan ${ }^{10-}$ 14 , which reported rates between $25.5 \%$ and $35.6 \%$ six weeks to six months after delivery of HDPcomplicated pregnancies.

Rates of persistent hypertension in pre-eclampsia of $39 \%$ and $18 \%$ at three months and two years postpartum, respectively, and higher mean blood pressure among pre-eclamptic cohort six months after delivery have also been reported in the Netherlands ${ }^{5,19}$. Switzerland reported $57.4 \%$ six to twelve weeks after delivery ${ }^{6}$, while United States reported $21 \%$ at six weeks postpartum ${ }^{20}$. Japanese women required more than two months after delivery for normalization of blood pressure following gestational hypertension and pre-eclampsia ${ }^{21}$. While these studies looked at either pre-eclampsia or gestational hypertension, the patterns and magnitude of prevalence of persistent hypertension across HDP sub-types and time from delivery appear common in subSaharan African women and others.

Although hypertension persists up to one year in many women in our study, the proportion and severity of hypertension progressively declined after delivery. The proportion of persistent hypertension, mean systolic and diastolic blood pressure progressively declined after delivery up to one year. This trend was also reported in Cameroonian study with hypertension prevalence declining from $42.8 \%, 27.8 \%$ and $14.8 \%$ respectively at six weeks, three months and six months postpartum, respectively ${ }^{11}$. As previously noted, elsewhere ${ }^{5}$, our study showed a significant proportion of women with persistent hypertension following HDP will not resolve after a year postpartum. In addition to a progression to develop chronic hypertension for previously normotensive women $^{7-8,31}$, this could also be explained by an underdiagnosis of pre-pregnancy chronic hypertension due to a lack of blood pressure measurements prior to pregnancy or a masking because of the first and second trimester physiologic blood pressure reduction? 
If women with HDP are not monitored long enough until blood pressure normalized or referred to continued medical practitioners' care, hypertension can persist unnoticed and increase the risk of adverse cardiovascular ${ }^{23,24}$, and renal complications (22\% prevalence of chronic kidney diseases has been reported among people with undiagnosed hypertension) ${ }^{26}$. Notably, we observed from unpublished data on adherence to quality of care for women with HDP that only one-thirds of women with hypertension in the first five days of delivery were on antihypertensive treatment. Although we did not assess this measure of compliance to treatment beyond this period, given previous evidence and the health care context ${ }^{27,28}$, it is unlikely to expect compliance would have improved over time.

Maternal age and BMI were predictors of persistent hypertension, as were observed in prior studies $^{11,18-19}$, and were expected based on the known associations between BMI, age and chronic hypertension. These have important clinical and public health implication given the mean age (28.9 years) and BMI $(28.8 \mathrm{~kg} / \mathrm{m} 2)$ among our cohort at ANC booking. While acknowledging that only $3 \%$ of our cohort registered for ANC within the first trimester when BMI measurement could have been more reliable, it is still concerning given the overweight and obesity prevalence among non-pregnant Nigerian women of $16.2 \%$ and $6.6 \%$, respectively ${ }^{29}$. The relationships between abnormal pre-pregnancy BMI and adverse feto-maternal outcomes have long been established ${ }^{30}$ and this finding strengthens a call for routine pre-conception care services, risk factors-based focused ANC and lifestyle modification for women in sub-Saharan Africa. BMI is a modifiable risk factor and women can be counselled to maintain healthy BMIs after delivery, and before future conceptions to reduce the risk of complications ${ }^{32}$, including subsequent persistent hypertension as shown in this study.

\section{Study strengths}

Our study was able to follow up nearly $70 \%$ of participants at one year postpartum; a reflection of extensive investments to retain women in the study within this context. This retention rate was also commendable based on recommended follow up rates between $50-80 \%$ for cohort studies $^{15-17}$. To our knowledge, this is the largest cohort of women with HDP followed up the longest in African settings (previous single-center studies in Cameroon, Nigeria, Sudan and 
Ugandan were for between six weeks to six months) $)^{10-14}$. This study enrolled women from eight tertiary health facilities spread across Nigeria ensuring diversity of patients and settings.

\section{Limitations}

Despite our reasonable follow-up rate, the missing 32\% of the women may have introduced limitations to the generalizability of the findings. However, analysis of sociodemographic and obstetrics characteristics between women who completed one year follow up and those lost (missed appointments) did not show difference between the two groups (Supplement table II). The lack of information of BP prior to pregnancy almost certainly lead to underestimation of chronic hypertension present before pregnancy. We used $4^{\text {th }}$ Korotkoff as diastolic BP which shows poor reproducibility in studies. Also, as women of higher socio-economic status (often of higher maternal age and BMIs compared to general maternal age) self-select themselves for routine ANC and delivery care at tertiary hospitals in Nigeria, this might may have caused overestimation of both determinants and outcomes.

\section{Clinical and research implications}

All women with HDP, irrespective of the sub-type, should be managed and followed up until hypertension is normalized either physiologically or supported by appropriate anti-hypertensive treatment ${ }^{1,2}$. In this study, over $60 \%$ of women with continuously elevated high blood pressure within the first week after delivery discontinued anti-hypertensive treatment. If this pattern is not reversed, the long-term cardiovascular and other poor health outcomes could be enormous. A large systematic review and meta-analysis has reported after pre-eclampsia complicated pregnancies a threefold higher risk for chronic hypertension (RR 3.70, 95\% Cl $2.70-5.05$ ) after 14.1 years weighted mean follow-up, a two-fold higher risk for ischemic heart diseases after 11.7 years (RR 2.16, 95\% Cl 1.86 - 2.52) nearly twofold higher risk of stroke (RR 1.81, 95\% Cl 1.45 2.27) after 10.4 years ${ }^{33}$.

The rising burden of cardiovascular diseases such as ischemic heart disease and stroke in subSaharan Africa underscores the importance of early recognition of women at risk ${ }^{23,25}$. As both hypertension and pre-eclampsia are independent risk factors for cardiovascular diseases ${ }^{24,28}$, adequate management of these risk factors can improve the future health of these women and 
reduce the burden to the health system. As such, HDP need to be included in guidelines for public health and individual health cardiovascular risk management (CVRM) strategies and risk models for women future cardiovascular health ${ }^{24}$. Importantly, beyond inclusion of postpartum management for women with HDP into existing guideline, effective implementation will be an essential challenge to overcome, especially in sub-Saharan African setting. Therefore, ongoing monitoring and evaluation would be necessary.

\section{Conclusion}

A substantial percentage of women who have had pregnancies complicated by hypertensive disorders have persistent hypertension beyond puerperium, and up to one year postpartum. Therefore, women with HDP-complicated pregnancies need regular postpartum evaluation until blood pressure is normalized or referral for further medical or primary care physician follow up including lifestyle modification, to reduce persistent hypertension and subsequent CVDs risk after delivery. These are strategies that may be effective, but still need to be evaluated for effectiveness after implementation on the reduction of HPD in a next pregnancy and long-term hypertension and CVD. Given the persistent burden of cardiovascular disease in pregnancy and pre-mature deaths of women globally, this will support the attainment of the Sustainable Development Goals.

Funding: This work was supported by the US Agency for International Development (USAID), under the terms of cooperative agreement number [USAID APSOAA-14-000048]. This article reflects the views of the authors and does not represent the views of the U.S. Government. S.M. Ishaku has been supported by the UMC Utrecht Global Health Fellowship Program.

Authors Contribution: All authors conceived the article. First and second authors developed the first draft. First and second authors performed the data analysis. All authors provide substantial review of the manuscript. 
SUPPLEMENT TABLE I: Laboratory protocol and standard for public tertiary hospitals in Nigeria

\begin{tabular}{|c|c|c|c|}
\hline S/N & ANALYTE/PARAMETER & ANALYTICAL PRINCIPLE & ANALYSER/EQUIPMENT/PLATFORM \\
\hline 1 & Glucose & $\begin{array}{l}\text { Trinder's Method: } \\
\text { Glucose Oxidase }\end{array}$ & Roche C311 and Abbott C4000 \\
\hline 2 & Sodium & $\begin{array}{l}\text { Ion Selective Electrode } \\
\text { (ISE) }\end{array}$ & Notek EL 3000 \\
\hline 3 & Potassium & ISE & Notek EL 3000 \\
\hline 4 & Chloride & ISE & Notek EL 3000 \\
\hline 5 & Bicarbonate & ISE & Notek EL 3000 \\
\hline 6 & Urea & $\begin{array}{l}\text { Berthelot's Method: } \\
\text { Urease Method }\end{array}$ & Roche C311 and Abbott C4000 \\
\hline 7 & Creatinine & $\begin{array}{l}\text { Modified Jaffe Kinetic } \\
\text { Method }\end{array}$ & Roche C311 and Abbott C4000 \\
\hline 8 & Uric acid & Uricase method & Roche C311 and Abbott C4000 \\
\hline 9 & Serum Total Calcium & $\begin{array}{l}\text { Cresophthalein } \\
\text { Complexone }\end{array}$ & Roche C311 and Abbott C4000 \\
\hline 10 & Inorganic Phosphate & $\begin{array}{l}\text { Ammonium } \\
\text { Phosphomolybdate } \\
\text { method }\end{array}$ & Roche C311 and Abbott C4000 \\
\hline 11 & Albumin & $\begin{array}{l}\text { Bromocresol Green } \\
\text { (BCG) }\end{array}$ & Roche C311 and Abbott C4000 \\
\hline 12 & AST & $\begin{array}{l}\text { Spectrophotometric } \\
\text { measurement of NADH } \\
\text { coupled Oxoacid } \\
\text { formation }\end{array}$ & Roche C311 and Abbott C4000 \\
\hline 13 & ALT & $\begin{array}{l}\text { Spectrophotometric } \\
\text { measurement of NADH } \\
\text { coupled Oxoacid } \\
\text { formation }\end{array}$ & Roche C311 and Abbott C4000 \\
\hline 14 & ALP & $\begin{array}{l}\text { Spectrophotometric } \\
\text { Using NPP as substrate }\end{array}$ & Roche C311 and Abbott C4000 \\
\hline 15 & Total Bilirubin & $\begin{array}{l}\text { Modified Diazo (Malloy } \\
\text { Evelyn) Method }\end{array}$ & Roche C311 and Abbott C4000 \\
\hline 16 & Direct Bilirubin & $\begin{array}{l}\text { Modified Diazo (Malloy } \\
\text { Evelyn/Jendrassic Groff) } \\
\text { Method }\end{array}$ & Roche C311 and Abbott C4000 \\
\hline
\end{tabular}


SUPPLEMENT TABLE II): Distribution of Sociodemographic and Obstetric Characteristic of HPD Clients that Were Followed up versus Loss to Follow up at one Year

\begin{tabular}{|l|l|l|}
\hline Variables & Followed up to 1 year & Loss to follow up at one year \\
\hline Mean age (SD) & $29.8(6.2)$ & $29.1(19.9)$ \\
\hline Mean Gestational age at onset of HDP (SD) & $33(9.9)$ & $33.5(7.3)$ \\
\hline Mean Gestational age at booking (SD) & $23.4(6.8)$ & $24.3(5.6)$ \\
\hline \multicolumn{2}{|l|}{} \\
\hline Category of HDP & \multicolumn{1}{l|}{} \\
\hline Chronic Hypertension n (\%) & $15(7.54)$ & $18(11.1)$ \\
\hline Gestational hypertension n (\%) & $52(26.1)$ & $20(12.4)$ \\
\hline Pre-eclampsia n (\%) & $107(53.8)$ & $92(56.8)$ \\
\hline Eclampsia n (\%) & $25(12.6)$ & $32(19.8)$ \\
\hline \multicolumn{2}{|l|}{} \\
\hline Mean BMI at baseline (SD) & $29.2(7.9)$ & $28.2(7.5)$ \\
\hline Mean BMI at 1 year (SD) & $28.7(7.2)$ & \\
\hline Mean systolic BP at baseline (SD) & $158.8(20.8)$ & $161.7(23.3)$ \\
\hline Mean systolic BP at 1 year (SD) & $133.3(26.6)$ & \\
\hline Mean diastolic at baseline (SD) & $137.1(23.4)$ & $135(29.3)$ \\
\hline Mean diastolic at 1 year (SD) & $86.1(18.3)$ & \\
\hline
\end{tabular}




\section{References}

1) Magee LA, Dadelszen PV; Stones W, Mathai M. An Evidence-based guide to monitoring, prevention and management. The FIGO textbook of pregnancy hypertension: 2016: 5-6

2) Brown MA, Magee LA, Kenny LC et al. Hypertensive disorders of pregnancy ISSHP Classification, Diagnosis, and Management Recommendations for International Practice. Hypertension. 2018; 72:24-43. DOI: 10.1161/HYPERTENSIONAHA.117.10803

3) Oladapo OT, Adetoro OO, Ekele BA et al. Nigeria Near-miss and Maternal Death Surveillance Network. When getting there is not enough: a nationwide cross-sectional study of 998 maternal deaths and 1451 near-misses in public tertiary hospitals in a lowincome country. Br J Obstet Gynaecol 2015; DOI: 10.1111/1471-0528.13450.

4) Rosemary T, Patrick $O^{\prime}$, Asma K. Current best practices in the management of hypertensive disorders in pregnancy. integrated Blood Pressure Control 2016:9 79-94

5) Berks D, Eric APS, Marek M, Willy V. Resolution of hypertension and proteinuria after preeclampsia. Obstet and Gynecol, 2009, 114(6):1307-1314

6) Agnes D, Wuerzner G, Ponte B et al. Prevalence of hypertensive phenotypes after preeclampsia: prospective cohort study. Hypertension2018; 71:103-109. DOI: 10.1161/HYPERTENSIONAHA.117.09799

7) Claire LS, Richard JM. What is the evidence base for diagnosing hypertension and for subsequent blood pressure treatment targets in the prevention of cardiovascular disease? BMC Medicine (2015) 13:256. DOI 10.1186/s12916-015-0502-5

8) Leening MJG, Ikram MA. Primary prevention of cardiovascular disease: The past, present, and future of blood pressure- and cholesterol-lowering treatments. PLoS Med (2018) 15(3): e1002539. https://doi.org/10.1371/journal.pmed.1002539

9) Gregory AR, Mark DH, Andrew EM et al. Global and Regional Patterns in Cardiovascular Mortality from 1990 to 2013. Circulation. 2015; 132:1667-1678. DOI: 10.1161/CIRCULATIONAHA.114.008720.)

10) Fadalallah ZM, Elhassan EM, Rayis DA, Abdullahi H, Adam I. Prospective cohort study of persistent hypertension following pre-eclampsia at Medani Hospital, Sudan. Int/ J Gynecol Obstet 2016; 134:66-68. 
11) Kaze FF, Njukeng FA, Andre-Pascal K et al. Post-partum trend in blood pressure levels, renal function and proteinuria in women with severe preeclampsia and eclampsia in SubSaharan Africa: A 6-months cohort study. BMC Pregnancy and Childbirth 2014; 14:134.

12) Nakimuli A, Elliott AM, Kaleebu P, Moffett A, Mirembe F. Hypertension Persisting after Pre-Eclampsia: A Prospective Cohort Study at Mulago Hospital, Uganda. PLoS ONE 2013; 8(12): e85273. doi: 10.1371/journal.pone.0085273

13) Ndayambagye EB, Nakalembe M, Kaye DK. Factors associated with persistent hypertension after puerperium among women with preeclampsia/eclampsia in Mulago hospital, Uganda. BMC Pregnancy and Childbirth 2010; 1471-2393/10/12

14) Olagbuji B, Ezeanochie M, Ande A, Okonkwo C. Prevalence and risk factors for persistent hypertension after the puerperium in pregnancies complicated with hypertensive disorders. J Obstet Gynaecol. 2012 Aug;32(6):529-32. doi: 10.3109/01443615.2012.689891

15) Kristman V, Manno M, Côté P. Loss to Follow-Up in Cohort Studies: How Much is Too Much? European Journal of_Epidemiology 2004; 19(8):751-60.

16) Lohr SL. Nonresponse. In: Lohr SL (ed.), Sampling: design and analysis. Pacific Grove: Duxbury Press, 1999: 255-287.

17) Altman DG. Statistics in medical journals: some recent trends. Stat Med 2000; 19: 32753289

18) Hwang J, Park S, Oh S et al. The risk factors that predict chronic hypertension after delivery in women with a history of hypertensive disorders of pregnancy. Medicine 2015; 94, (42): e1747

19) van RBB, Nijdam ME, Bruinse HW et al. Cardiovascular disease risk factors in women with a history of early-onset preeclampsia. Obstet Gynecol. 2013;121(5):1040-8. doi: 10.1097/AOG.0b013e31828ea3b5.

20) Levine LD, Nkonde-Price C, Limaye M, Srinivas SK. Factors associated with postpartum follow-up and persistent hypertension among women with severe preeclampsia. $J$ Perinatol 2016; 36(12): 1079-1082. doi:10.1038/jp.2016.137. 
21) Mikami $Y$, Takagi $K$, Itaya $Y$ et al. Post-partum recovery course in patients with gestational hypertension. J Obstet Gynecol Res 2014; 40(4):919-25. doi: 10.1111/jog.12280.

22) World Health Organization. Cardiovascular diseases. Geneva, Switzerland: World Health Organization; Available from http://www.who.int/mediacentre/factsheets/fs317/en/.

23) Suzan H, Wim G, Milena P. Trends in cardiovascular diseases and associated risks in subSaharan Africa: a review of the evidence for Ghana, Nigeria, South Africa, Sudan and Tanzania, The Aging Male, 2019. 22:3, 169-176, DOI: 10.1080/13685538.2019.1582621

24) Guidelines for the Prevention of Stroke in Women: A Statement for Healthcare Professionals from the American Heart Association/American Stroke Association. Stroke. 2014; 45:1545-1588.

25) Jean JN, Jean JB, Ulrich FN et al. The burden of hypertensive disorders in Africa: A systematic review and meta-analysis. J Clin Hypertens. 2019; 21:479-488.

26) Deidra CC, Laura CP, Edgar RM et al. Prevalence of Chronic Kidney Disease in Persons with undiagnosed or prehypertension in the United States. Hypertension. 2010; 55(5):11021109

27) Elysia La, Miriam R, Godfrey MM, Redempta M, Margaret EK. Missed opportunities to improve the health of postpartum women: high rates of untreated hypertension in rural Tanzania. Matern Child Health J. 2017; 21(3): 407-413.

28) Boateng $D$, Wekesah F, Browne JL et al. Knowledge and awareness of and perception towards cardiovascular disease risk in sub-Saharan Africa: A systematic review. PLOS ONE (2017) 12(12): e0189264. https://doi.org/10.1371/ journal. pone.0189264

29) Ghose B, Yaya S. Media use and excess body weight among women in Nigeria: a crosssectional study. BMJ Open 2018;8: e020802. doi:10.1136/bmjopen-2017-020802

30) Joice MV, Jusciele BM, Claudia GM et al. Maternal and fetal outcomes in pregnancies complicated by overweight and obesity. Reproductive Health (2016) 13:100. DOI 10.1186/s12978-016-0206-0

31) Reem M, Sana A, Anu G, Rocco CV. A Comprehensive Review of Hypertension in Pregnancy. Journal of Pregnancy; Volume 2012, doi:10.1155/2012/105918 
32) Adane AA, Mishra GD, Tooth LR. Adult Pre-pregnancy Weight Change and Risk of Developing Hypertensive Disorders in Pregnancy. Paediatr Perinat Epidemiol. 2017 ;31(3):167-175. doi: 10.1111/ppe.12353.

33) Bellamy L, Casas JP, Hingorani AD, Williams DJ. Pre-eclampsia and risk of cardiovascular disease and cancer in later life: Systematic review and meta-analysis. BMJ. 2007; 335 (7627):974

34) Angela T, Cristiano F. Diagnosis of hypertensive disorders in pregnancy: an update. Journal of Laboratory and Precision Medicine. doi: 10.21037/2019.11.04

35) Magee LA, Sharma S, Nathan HL et al. The incidence of pregnancy hypertension in India, Pakistan, Mozambique, and Nigeria: A prospective population-level analysis. PLoSMed 16(4): e1002783.https://doi.org/2019/10.1371/journal.pmed.1002783

36) Arie F, Joris AMV, Gert AVM, Hein WB, Gerard HAV. The fourth sound of Korotkoff in pregnancy: a myth? The Lancet (1996). 347: 841 


\title{
Chapter 5
}

\section{METABOLIC SYNDROME FOLLOWING HYPERTENSIVE DISORDERS IN PREGNANCY IN A LOW-RESOURCE SETTING: A COHORT STUDY}

\author{
Salisu M Ishaku* \\ Tunau Karima* \\ Khadijat A Oboirien \\ Agbo P Innocent \\ Oyeneyin Lawal \\ Tukur Jamilu \\ Joyce L Browne \\ Kayode A Gbenga \\ Onyebuchi K Azubuike \\ Dattijo M Lamaran \\ Aminu M Baffah \\ Owa O Olorunfemi \\ Odusolu O Patience \\ Warren E Charlotte \\ Hanifah D Abubakar \\ Abdulkarim Musa \\ Kerstin-Klipstein Grobusch \\ Arie Franx \\ Diederick E Grobbee
}

*First and second authors contributed equally

Submitted to Pregnancy Hypertension Journal, responded to reviewers' comments, and awaiting decision 


\section{Background}

Hypertensive disorders in pregnancy (HDP) are associated with increased risk of future medical conditions like metabolic syndrome. Despite the huge burden of HDP in sub-Saharan Africa, its association with metabolic syndrome has not been adequately studied in this population.

\section{Methods}

This was a prospective cohort study on pregnant women recruited between August 2017 and April 2018 up to one year after their deliveries and evaluated for presence of metabolic syndrome at delivery, nine weeks, six months and one year. Descriptive and logistic regression analyses were performed to compare baseline, outcomes and incidence rates for women with HDP and normotensive cohorts well as identification of factors that predict metabolic syndrome.

\section{Results}

A total of 488 pregnant women were included in the cohort: 410 and 78 with hypertensive disorders and normotensive, respectively. None of the normotensive had metabolic syndrome at delivery and up till one year afterwards $(1.7 \%=1$ out of 59 observations). The prevalence of metabolic syndrome among those with HDP were $17.4 \%$ (71 of 407), 8.7\% (23 of 263), 4.7\% (11 of 232 ) and $6.1 \%$ (17 of 278), at delivery, nine weeks, six months and one year postpartum, respectively. High BMI and blood pressure were the main drivers of metabolic syndrome in this population. The incidence rate for metabolic syndrome in HDP versus normotensive at one year were, respectively, 57.5/1000 person years (95\% $\mathrm{Cl}$; $35.8-92.6)$. and 16.9/1000 person years $(95 \% \mathrm{Cl} ; 2.4-118.3)$, with incidence rate ratio of $3.4 / 1000$ person years. Only parity significantly predicted the presence of metabolic syndrome for women with HDPs at one year in the multivariable logistic analysis [(aOR=3.26/delivery $(95 \% \mathrm{Cl} ; 1.21-8.79)]$. 


\section{Conclusion}

Women with HDP were associated with a higher incidence of metabolic syndrome up to one year after delivery. All women with HDP, especially those of higher parity should, therefore, be routinely screened for metabolic syndrome within the first year of delivery to reduce future cardiometabolic health risks.

Key words: Hypertensive disorders in pregnancy, metabolic syndrome, Nigeria 


\section{Introduction}

Metabolic syndrome is a group of clinical, metabolic and biochemical abnormalities with negative impact on global health ${ }^{1}$. Based on the International Diabetes Federation (IDF) consensus statement, it is defined as the presence of body mass index $>30 \mathrm{~kg} / \mathrm{m}^{2}$ with any two of the following: triglycerides $\geq 1.7 \mathrm{mmol} / \mathrm{L}$, high-density lipoprotein $<1.29 \mathrm{mmol} / \mathrm{L}$, hypertension (systolic BP $\geq 130$ or diastolic BP $\geq 85 \mathrm{~mm} \mathrm{Hg}$ ) or fasting blood glucose $\geq 100 \mathrm{mg} / \mathrm{dL}$ or 5.6 $\mathrm{mmol} / \mathrm{L}^{2}$. Normal pregnancy adaptations to cater for nutritional needs of the growing fetus induces cardiometabolic stress reminiscent of metabolic syndrome with increased insulin resistance, hyperlipidemias and changes in protein and amino acid metabolism ${ }^{3,9}$. As such, pregnancy is sometimes considered a 'stress test'. Hypertensive disorders in pregnancy (HDP) may therefore be manifestations of underlying metabolic and cardiovascular disease (CVD) risks due to constitutional predisposition ${ }^{4-8,10}$.

There is growing evidence that abnormalities in cardiometabolic markers during pregnancy can persist in HDP including pre-eclampsia well beyond the peri-partum period ${ }^{4,9-10}$. In fact, metabolic syndrome has been shown to be commoner among these women ${ }^{4,7,10}$. In addition, HDP without initial aberrations may begin to exhibit abnormal metabolic profiles postpartum, some of which result in metabolic syndrome ${ }^{10}$. Consequently, many authorities, including the American Heart Foundation, have included HDP as indicator risk factors for cardiovascular diseases (CVDs) ${ }^{8}$. The International Society for the Study of Hypertension in Pregnancy (ISSHP) and the National Institute for Health and Care Excellence (NICE) guidelines also recommend longterm postpartum follow up for women with HDP due to heightened cardiovascular and metabolic disease risks associated with prior HDP ${ }^{11,12}$.

Following HDP, varying prevalence rates of metabolic syndrome have been reported from around the world depending on the postpartum interval the evaluation was made, with values ranging from $13.9 \%$ in the Netherlands (median of 8 months postpartum) and $14.5 \%$ in the United States of America ${ }^{9,13}$. Fourteen years after pre-eclampsia, an $82.3 \%$ prevalence of metabolic syndrome has been reported in Brazil ${ }^{14}$. Udenze et al. reported a prevalence range from $10.9 \%$ to $27.3 \%$ after a pre-eclamptic pregnancy in an inter-continental systematic review ${ }^{15}$. 
Evidence on the burden of metabolic syndrome in prior HDP in sub-Saharan African setting is limited. One study from Ghana was conducted during the second half of pregnancy and reported a prevalence of $61 \%$ based on the National Cholesterol Education Program (NCEP) definition ${ }^{16}$. Two Nigerian cross-sectional studies are also available with reported prevalence of metabolic syndrome ranging from of $24.3 \%$ to $31.7 \%^{17,18}$. However, they were conducted among semiurban non-pregnant women.

Given that metabolic syndrome is considered an intermediate and modifiable risk factor for future cardiovascular and metabolic diseases and its proven association with prior HDP, a prospective cohort was conducted among women with prior HDP who delivered at tertiary facility settings, in order to determine the prevalence and risk factors of metabolic syndrome.

\section{Methods}

\section{Study design}

This was a prospective cohort study on pregnant women recruited between August 2017 and April 2018 up to one year after deliveries. They were evaluated for metabolic syndrome at predetermined intervals and the last recruited participant completed the study on March 31, 2019.

\section{Study setting}

The study was conducted in eight tertiary hospitals in Nigeria namely: Abubakar Tafawa Balewa University Teaching Hospital, Bauchi State, University of Calabar Teaching Hospital, Cross River State, Federal Teaching Hospital, Ebonyi state, Federal Medical Center, Kogi State, Aminu Kano Teaching Hospital Kano State, Usmanu Danfodiyo University Teaching Hospital Sokoto as well as Mother and Child Hospital and the University of Medical Sciences Teaching Hospital, both in Ondo State. The health facilities were high-volume sites with well-functioning clinics, delivery rooms and laboratory services with combined annual deliveries averaging 38,400.

\section{Participants}

This study recruited women 18 years old and above who delivered with diagnoses of HDP. Multiple pregnancies and medical disorders in pregnancy other than hypertensive disorders such 
as diabetes mellitus, sickle cell anemia, heart disease, kidney and other connective tissues disorders were excluded. The normotensive cohort comprised of pregnant women who also delivered in the study sites.

\section{Study procedures}

Participants (both women with HDP and normotensive) were informed of the study during the antenatal period (for registered clients) or after delivery but all recruitments were done within 24 hours of delivery. Intending participants were individually counseled, informed consent obtained - either signed or thumb printed - (consent rate over 95\%) and enrollment forms (comprising information on socio-demographic and obstetric variables) was completed. After enrollment, the women underwent baseline clinical evaluations to assess their symptoms and signs. In addition, laboratory investigations were performed before discharged from the hospitals. They were subsequently followed-up during which the same clinical and laboratory investigations conducted at baseline were repeated at nine weeks, six months and one year postpartum. To enhance follow up, the participants were requested to provide their contact information (particularly personal and spousal mobile telephone numbers). They were reminded of their follow up appointments through phone calls. Participants' contact information was not linked to their clinical records while all clinical information was linked to unique identifiers.

\section{Exposure variables}

The main exposures of interest were the presence of any HDP sub-type namely, chronic hypertension, gestational hypertension and pre-eclampsia as defined by the ISSHP ${ }^{11}$. Hypertension was defined as systolic blood pressure of $\geq 140 \mathrm{mmHg}$ and or diastolic blood pressure of $\geq 90 \mathrm{mmHg}$ measured on two consecutive periods 4-6 hours apart. Chronic hypertension in pregnancy was defined as any hypertension with onset before the index pregnancy or diagnosed within the first 20 weeks of the index pregnancy. Gestational hypertension was defined as any hypertension occurring after the first 20 weeks of pregnancy without significant proteinuria ( $<2++$ of proteinuria on urine dipstick measurement) or any hematological or biochemical abnormality. Pre-eclampsia was defined as hypertension with 
onset after the first 20 weeks of pregnancy with significant proteinuria $(\geq 2++$ of proteinuria on urine dipstick measurement) or the presence of any hematological and biochemical abnormality.

\section{Outcome variables}

We assessed the prevalence and incidence of metabolic syndrome at three time-periods after delivery: nine weeks, six months and one year. Metabolic syndrome was as defined by the International Diabetes Federation ${ }^{2}$, the presence of $\mathrm{BMI}>30 \mathrm{~kg} / \mathrm{m}^{2}$ with any two of; triglycerides $\geq 1.7 \mathrm{mmol} / \mathrm{L}$, high-density lipoprotein $(\mathrm{HDL})<1.29 \mathrm{mmol} / \mathrm{L}$, hypertension (systolic BP $\geq 130$ or diastolic BP $\geq 85 \mathrm{~mm} \mathrm{Hg}$ ) or fasting blood glucose $\geq 100 \mathrm{mg} / \mathrm{dL}$ or $5.6 \mathrm{mmol} / \mathrm{L}$ We used the IDF definition for ease of use in our setting as other commonly used definitions require measurements not readily available such as measures of insulin resistance, insulin concentration (the World Health Organization criteria) and waist circumference (the National Cholesterol Education Program - NCEP ATP III) ${ }^{19}$.

\section{Data source/data collection}

At each data collection period, participant's BMI (weight $(\mathrm{kg}) /$ height $\left(\mathrm{m}^{2}\right)$ ), was measured along with urine protein estimation (using meditest combi 10 dipsticks) and blood pressure (BP) by auscultation technique using mercury sphygmomanometer. The first and fourth Korotkoff sounds were taken as systolic and diastolic values, respectively. A general physical examination for signs was also conducted. In addition, blood samples were collected for laboratory tests which included fasting blood glucose and serum lipids (total cholesterols, HDL and low-density lipoproteins (LDL) cholesterols, triglycerides). All clinical examinations and blood sample collection were performed by trained medical officers (research assistants). Laboratory investigations were performed by scientists based on laid down protocols following standard practices used in all the study sites.

\section{Risk of bias}

The women with HDP and the normotensive cohort were recruited from a similar maternity population. In Nigeria, access to such health facilities is based on women's preferences, places of residence and socio-economic status (not necessarily based on referrals), with the more 
educated and high-income group typically attending tertiary care centers. Because women of particular risk strata could self-select themselves for tertiary care delivery, estimates of metabolic syndrome in this population may not be generalized. However, recruitment proceeded independently in all facilities. Case identification was done by specially trained midwives using standard diagnostic criteria. Outcomes assessors were blinded of participants' HDP status. The results of medical and laboratory investigations were entered in the electronic data capturing platform by trained research assistants as soon as the data were available to reduce the incidence of missing data. The hard copies of all medical and laboratory results were retained as source documents at the facilities for future reference when necessary.

\section{Sample size}

Given that on average $14 \%$ of pregnant women develop metabolic syndrome postpartum across previously conducted studies with varying follow up time ${ }^{9,13}$, it is estimated that 185 women in each arm were required to participate in the study in order to detect a similar level of postpartum metabolic syndrome in our Nigerian population after HDP and compare it to women after normotensive pregnancy, at alpha level of $5 \%$ and power of $80 \%$. Considering a potential $10 \%$ loss to follow up, 204 women were needed. As the study was only observational with minimal risks, many women as could consent to participation within available resources and time, were enrolled.

\section{Data analysis plan/statistical methods}

The women with HDP and the normotensive followed-up for each period were successfully analyzed. Laboratory results reported in $\mathrm{mg} / \mathrm{dl}$ were converted to $\mathrm{mmol} / \mathrm{L}$ before the analyses were carried out. Frequencies, percentages, proportions and means (standard deviations) were used to describe participants' obstetric and demographics variables as well as the prevalence of metabolic syndrome. Independent t-test was used to compare mean differences between the HDP and the normotensives. For comparing categorical data, chi square or Fisher's exact test was used. In addition, the incidence rates of metabolic syndrome (in person years experienced) for each period were calculated to delineate between new onset metabolic syndrome attributable to hypertensive disorders from the persistent effects of pregnancy adaptation that could 
influence prevalence. Univariable and multivariable logistic regression analyses to identify factors that predict metabolic syndrome at six months and one year postpartum were performed. Confounders' selection was hypothesis-driven, and the following variables were included: age, parity, gestational age at delivery, gestational age at onset of HDP, booking status, gestational hypertension, pre-eclampsia and eclampsia. The data was analyzed using SPSS IBM version 25.0.

\section{Results}

In this cohort, 410 women with hypertensive disorders and 78 normotensives ones (due to differential consenting rates between the two cohorts (95\% and $38 \%$ for HDPs and normotensive respectively)) participated. Figure 1 shows flowchart of follow up of women with HDPs versus the normotensive over the one-year period. The number of women assessed at various time points varied due to missed appointment: 263 (65\%), 232 (57\%), $278(68 \%)$ and 53 (68\%), 43 (55\%), and 58 (74\%) for HDPs and control at nine weeks, six months and one year, respectively. 
Figure 1: Follow-up rates among women HDP and the normotensive pregnancies
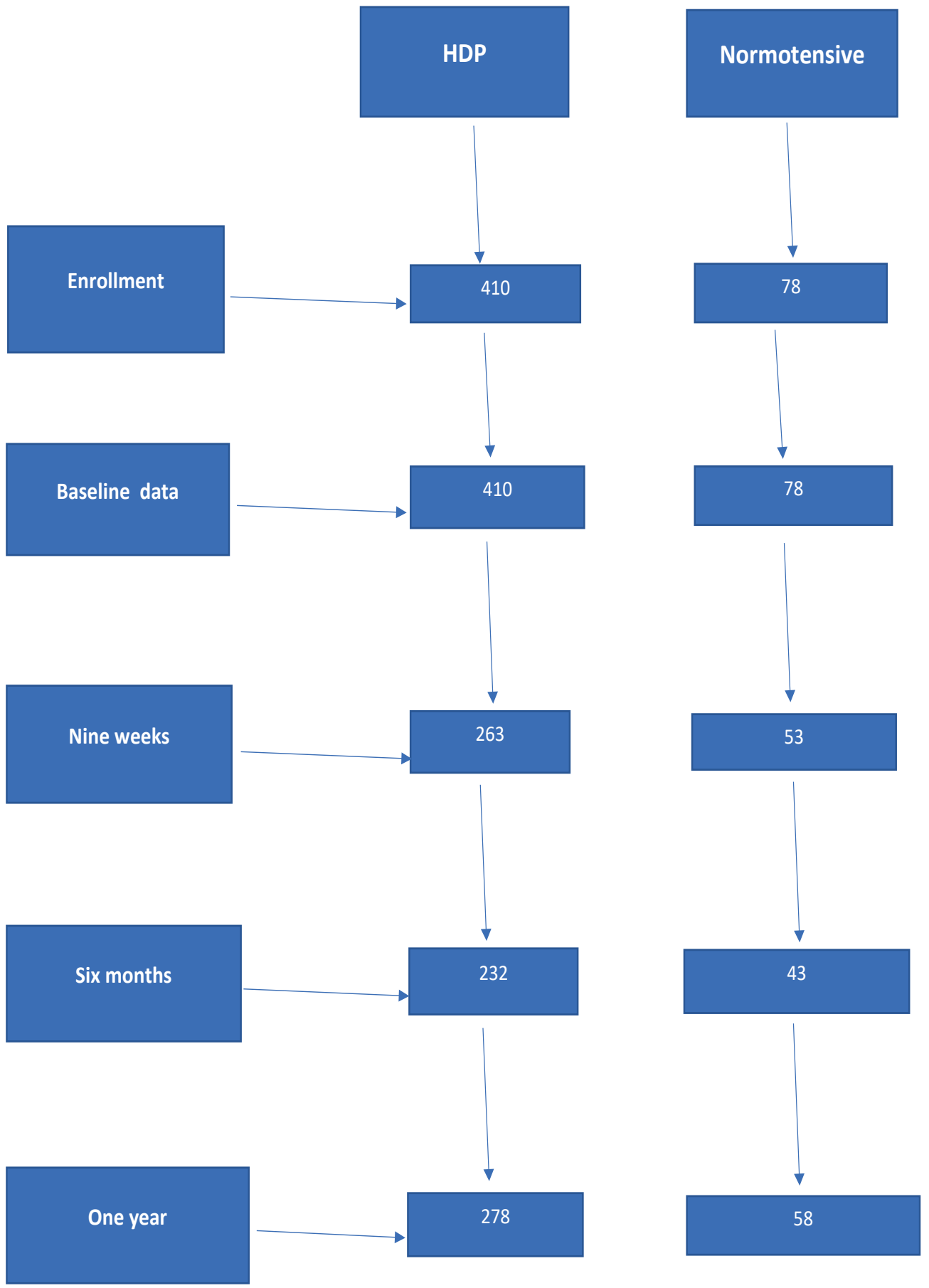
Table 1 shows the baseline characteristics of the women. The mean age and booking BMI of women with HDP and normotensive women were similar [age: (29.4; $\mathrm{Sd}=0.58)$ versus (28.3; $\mathrm{Sd}=0.59), \mathrm{BMI}$ : (28.8; $\mathrm{Sd}=0.51)$ versus (26.7; $\mathrm{Sd}=0.81)$ ]. The proportion of women who were primigravidae was higher in the HDP group $(21 \%, n=86 / 410)$ than in the normotensive counterpart (13\%, n=10/78). About 40\% (163) and 10\% (8) of the HDP and the normotensive women, were not registered for antenatal care (ANC). Of those who registered, similar proportions of HDP and normotensives did so within the first trimester $(3.4 \%, n=14$ versus $3.8 \%$, $\mathrm{n}=3)$. The mean gestational age at booking were similar (23.7 ( $\mathrm{Sd}=0.41)$ versus $22.8(\mathrm{Sd}=0.75))$. Women with HDP were more likely to deliver preterm compared to normotensive women (mean delivery gestational age of $36.5(\mathrm{Sd}=4.1)$ versus $38.3(\mathrm{Sd}=1.7)]$.

At baseline (within 24 hours of delivery, Table II), women with HDP significantly differed from the normotensive in some components of metabolic syndrome. HDP had significantly higher mean BMI [29.1 kg/m² $(S d=7.3)$ versus $\left.27.3 \mathrm{~kg} / \mathrm{m}^{2} \quad(S d=6.2) \mathrm{p}=0.047\right]$, mean systolic [159.6mmHg $(\mathrm{Sd}=22.2)$ versus $113.7 \mathrm{mmHg}(\mathrm{Sd}=10.9), \mathrm{p}=0.001$ ] and mean diastolic blood pressure [102 $\mathrm{mmHg}$ $(S d=15.8)$ versus $71.5 \mathrm{mmHg}(S d=6.9), p=0.001$ ], with no significant differences with respect to serum cholesterol, triglycerides and fasting blood glucose levels, although the proportions of women with high abnormal values for these three components are higher among the HDP at baseline and at 1 year (with the exception of FBS at baseline). Similarly, at one year after delivery, women with HDP as compared to the normotensives had significantly higher mean BMI [28.5 $\mathrm{kg} / \mathrm{m}^{2}(\mathrm{Sd}=7.1)$ versus $26.4 \mathrm{~kg} / \mathrm{m}^{2}(\mathrm{Sd}=5.5), \mathrm{p}=0.035$ ], mean systolic [132.86mmHg $(\mathrm{Sd}=26.8)$ versus $112.07 \mathrm{mmHg}(\mathrm{Sd}=13.8), \mathrm{p}=0.001$ ] and mean diastolic blood pressure [86.8mmHg ( $\mathrm{Sd}=18.5$ ) versus $72.6 \mathrm{mmHg}(\mathrm{Sd}=11.5), \mathrm{p}=0.001]$, but similar cholesterol, triglycerides and fasting blood glucose concentrations. At both the baseline and at 1 year postpartum, higher proportion of women with HDP, as compared to the normotensive, had abnormality in all the components of metabolic syndrome (Table II)

Women with normotensive pregnancies had no metabolic syndrome at baseline, nine weeks and six months after delivery, but $1.7 \%(n=1 / 59)$ had it at one year postpartum. For women with HDP, 
this was $17.4 \%(71 / 410), 8.7 \%(15 / 263), 4.7 \%(11 / 232)$ and $6.1 \%(17 / 278)$, respectively. The incidence rates for metabolic syndrome among women with HDP dropped from 329.7/1000 person years (95\% Cl; 217.8 - 498.9) to 57.5/1000 person years at one year. For women with a normotensive pregnancy the incidence rate at one year was $16.9 / 1000$ person years $(95 \% \mathrm{Cl} ; 2.4$ $-118.3)$. 


\begin{tabular}{|c|c|c|c|c|c|c|c|c|c|c|c|c|c|c|c|}
\hline 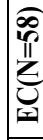 & & 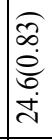 & $\begin{array}{l}\widehat{\widehat{2}} \\
\hat{e} \\
\text { } \\
\dot{\nu}\end{array}$ & & 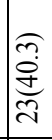 & 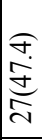 & 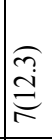 & & 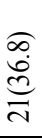 & 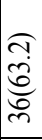 & 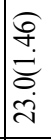 & & $\begin{array}{l}\widetilde{n} \\
\stackrel{n}{n}\end{array}$ & శ్ & 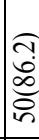 \\
\hline 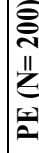 & & 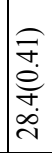 & 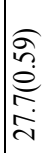 & & 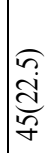 & 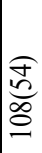 & $\begin{array}{l}\widetilde{\Omega} \\
\widetilde{d} \\
\stackrel{f}{f}\end{array}$ & & 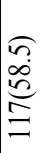 & 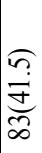 & 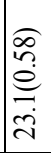 & & $\frac{\widetilde{\Omega}}{\stackrel{2}{\sim}}$ & 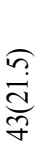 & 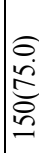 \\
\hline 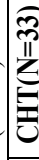 & & 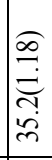 & 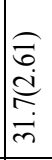 & & $\begin{array}{l}\partial \\
\stackrel{c}{c}\end{array}$ & 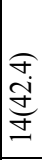 & $\begin{array}{l}\widehat{\sigma} \\
\stackrel{0}{0} \\
\infty \\
-\end{array}$ & & 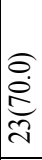 & 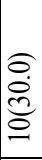 & 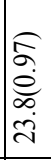 & & & 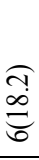 & $\begin{array}{l}\infty \\
\frac{\infty}{\infty} \\
\stackrel{\infty}{\sim}\end{array}$ \\
\hline 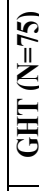 & & $\begin{array}{c}\stackrel{f}{m} \\
\tilde{n} \\
\tilde{m} \\
\tilde{m} \\
\tilde{n}\end{array}$ & 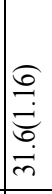 & & 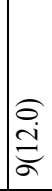 & 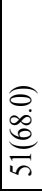 & 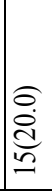 & & $\begin{array}{l}\text { } \\
\dot{\otimes} \\
\dot{0} \\
\dot{0}\end{array}$ & 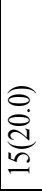 & 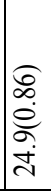 & & 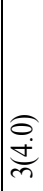 & 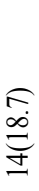 & 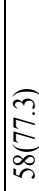 \\
\hline A $^{\prime}$ & & 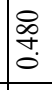 & $\stackrel{8}{0}$ & ণิ & & & & 这 & & & $\begin{array}{l}\stackrel{8}{0} \\
\stackrel{1}{0} \\
0\end{array}$ & 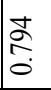 & & & \\
\hline & 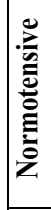 & 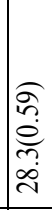 & 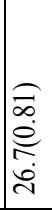 & & 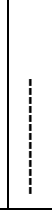 & 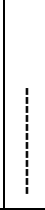 & 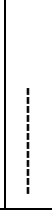 & & 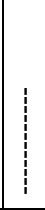 & & 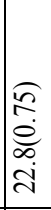 & & & & \\
\hline 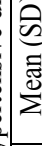 & 宣 & 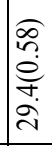 & $\begin{array}{l}\widehat{\vec{n}} \\
\stackrel{e}{e} \\
\infty \\
\infty \\
\infty \\
i\end{array}$ & & 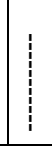 & 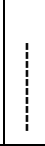 & 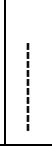 & & 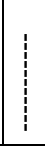 & & 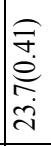 & & & & \\
\hline & 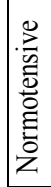 & & & & 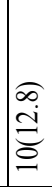 & 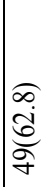 & $\begin{array}{l}\stackrel{\Im}{J} \\
\stackrel{d}{d} \\
\precsim\end{array}$ & & 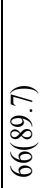 & 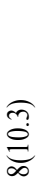 & $\mid$ & & $\begin{array}{l}\infty \\
\dot{d} \\
m \\
m\end{array}$ & 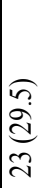 & $\begin{array}{l}\hat{\text { fo }} \\
\text { o } \\
\text { in }\end{array}$ \\
\hline है & $\hat{\hat{\mid}}$ & & & & 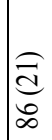 & 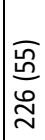 & 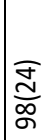 & & 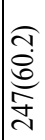 & 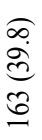 & 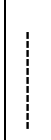 & & 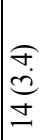 & $\underset{\stackrel{\infty}{\infty}}{\stackrel{\infty}{\Xi}}$ & $\begin{array}{l}\stackrel{2}{\hat{\sigma}} \\
\approx \\
\curvearrowright\end{array}$ \\
\hline 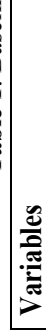 & & 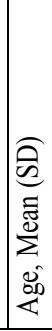 & 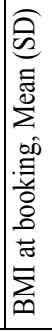 & है & 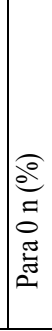 & 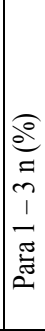 & 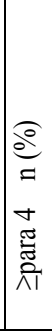 & 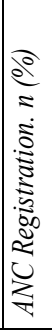 & 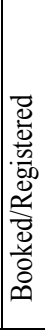 & 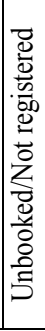 & 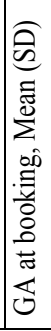 & 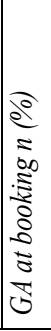 & 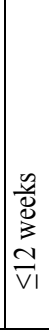 & 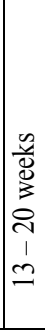 & 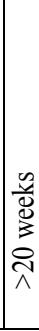 \\
\hline
\end{tabular}




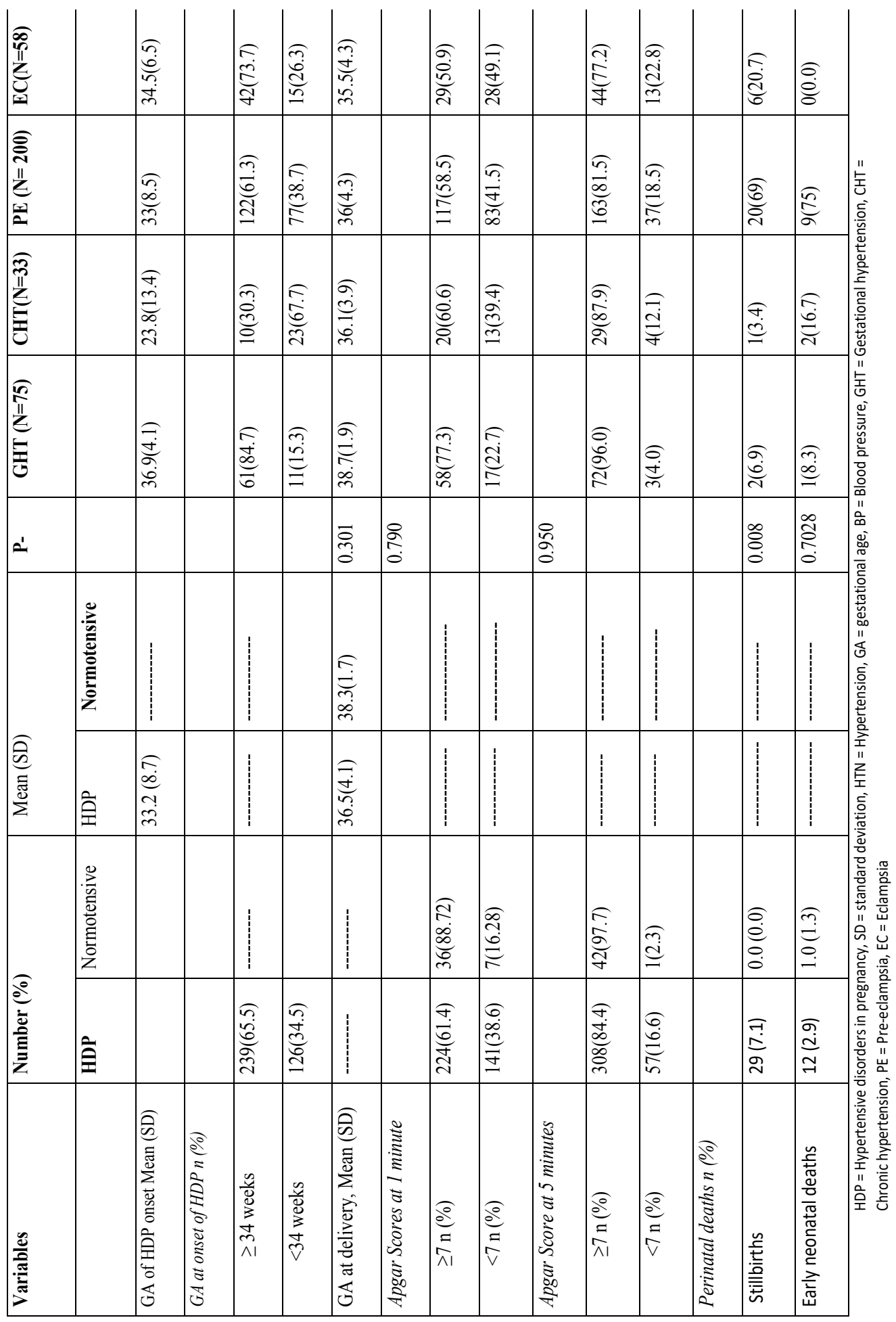



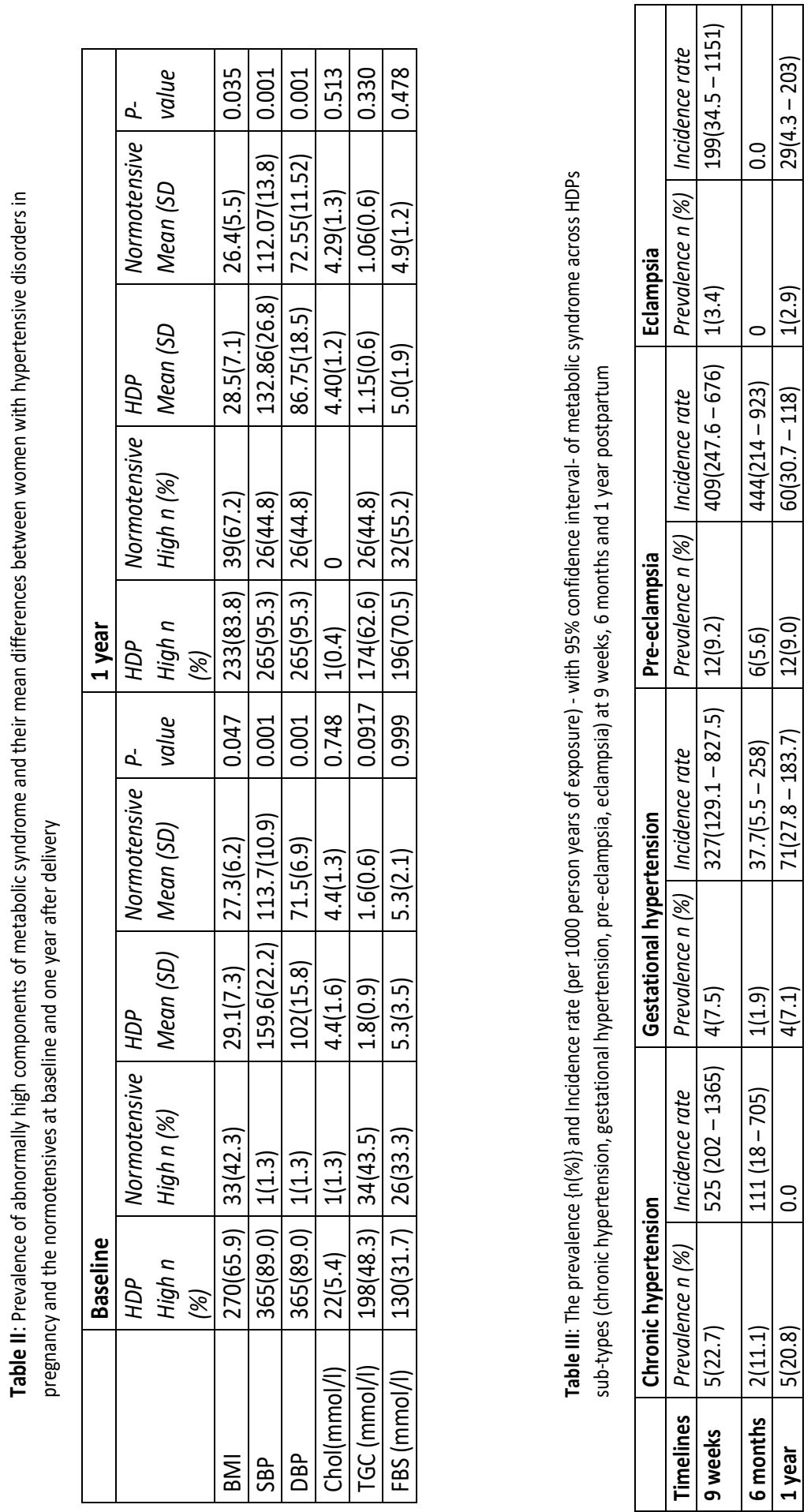


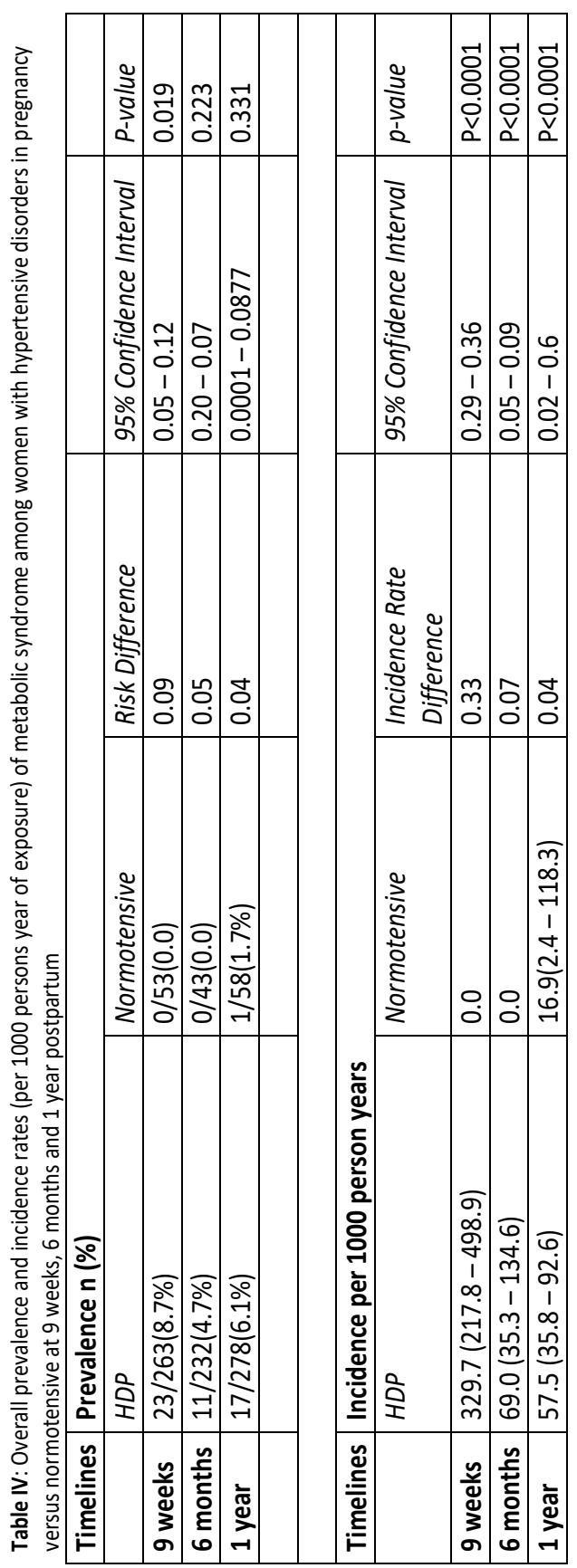


The incidence and prevalence rates of metabolic syndrome among the different HDP categories differed (Table III). At 6 months postpartum, women with pre-eclampsia were more likely to have metabolic syndrome $[(I R=444 / 1000$ person years $(95 \% \mathrm{Cl} ; 214-923)$, followed by chronic hypertension $[(I R=111 / 1000$ person years $(95 \% \mathrm{Cl} ; 18-705)$ and finally gestational hypertension $[(I R=37.7 / 1000$ person years $(95 \% \mathrm{Cl} ; 5.5-258)]$. While no woman with chronic hypertension developed new onset metabolic syndrome at one year, the rate in gestational hypertension, preeclampsia and eclampsia were, respectively, 71/1000 person years $(95 \% \mathrm{Cl} ; 27.8-183.7)$, $60 / 1000$ person years $(95 \% \mathrm{Cl} ; 30.7-118)$ and $29 / 1000$ person years $(95 \% \mathrm{Cl} ; 4.3-203)$.

Table IV describes the overall prevalence and incidence rates of metabolic syndrome for the HDP and normotensive at each of the time points. At nine weeks, six months and one year postpartum, the prevalence of metabolic syndrome was higher in the HDP category than in the normotensive but only significantly so at nine weeks ( $p-0.019)$. Similarly, development of new onset metabolic syndrome (incidence) occurs significantly more among the HDP at all postpartum times with $\mathrm{p}<0.0001$.

while Table $\mathbf{V}$ describes the results of univariable and multivariable logistic regression analyses of maternal demographic and obstetric factors as predictors of metabolic syndrome at six months and one year postpartum. At six months postpartum, none of the possible predictors were associated with metabolic syndrome. At one year postpartum, only parity significantly predicted the development of metabolic syndrome [(aOR=3.26/delivery $(95 \% \mathrm{Cl} ; 1.21-8.79)]$. 


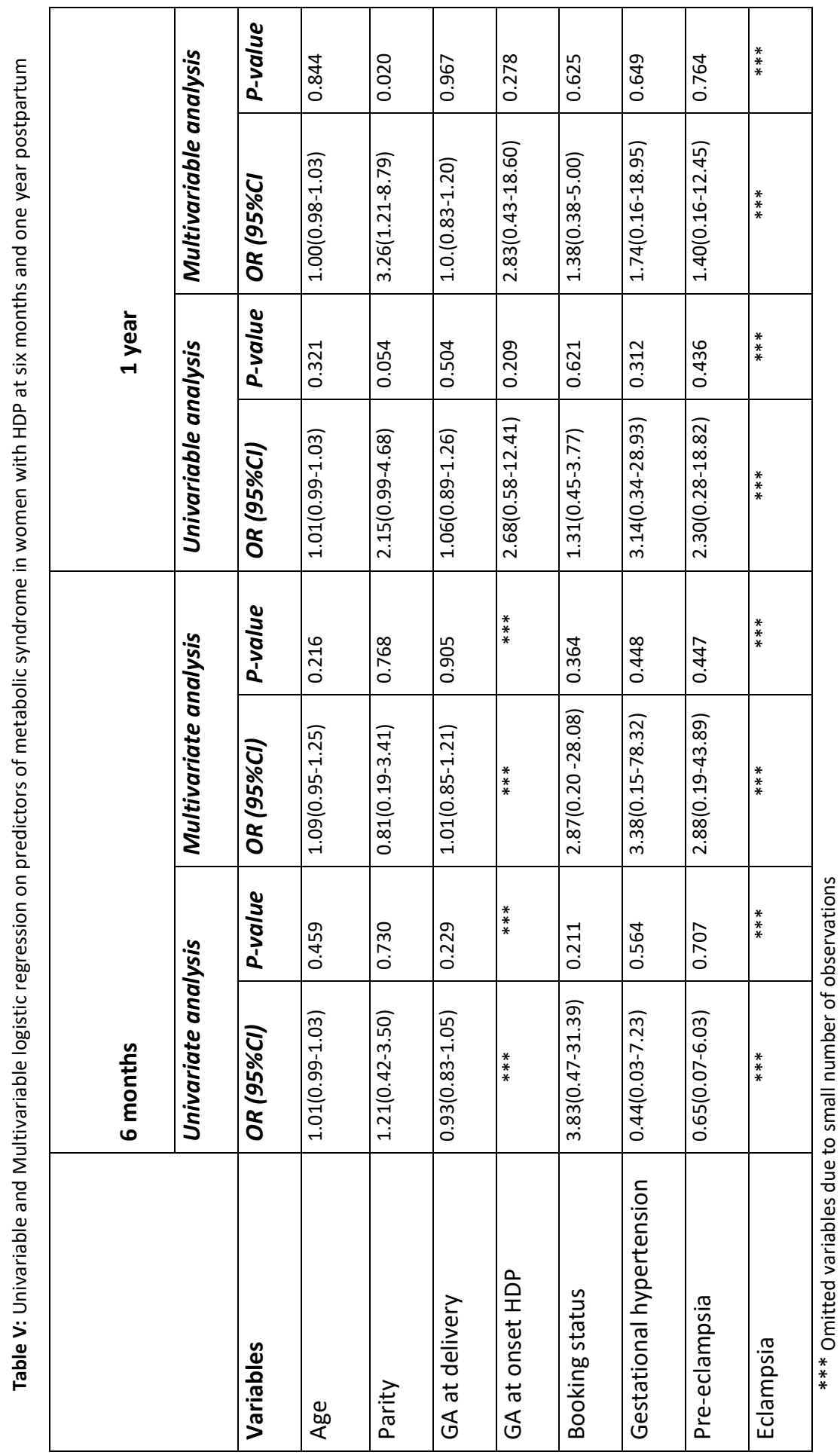




\section{Discussion}

This study showed a relatively high prevalence (6.1\%) of (persistent) metabolic syndrome up to one year postpartum. In contrast, only $1.7 \%$ of women in the comparison group developed metabolic syndrome the year following birth. The prevalence of metabolic syndrome after puerperium among our cohort of women with HDPs was lower than the reported global proportion of about $15 \%^{2,9,13}$. These differences may be reflective of differences in the criteria used and the postpartum time periods during which measurements were taken. The IDF criteria was used in this study, whereas others used the NCEP ATP III (defined as presence of any three of the following: hypertension $\geq 130 / 85 \mathrm{mmHg}$; fasting plasma glucose $\geq 6.1 \mathrm{mmol} / \mathrm{L}$; fasting plasma triglycerides $\geq 1.69 \mathrm{mmol} / \mathrm{L}$; fasting $\mathrm{HDL}$ cholesterol $<1.29 \mathrm{mmol}$ or subjects were receiving treatment for their condition and waist circumference $>88 \mathrm{~cm}$ ) and the World Health Organization definition (hyperinsulinemia - the upper fourth of the fasting insulin level among nondiabetic subjects or hyperglycemia - fasting glucose $\geq 110 \mathrm{mg} / \mathrm{dl}$ - in addition to at least two of the following: waist girth $\geq 94 \mathrm{~cm}$, dyslipidemia - triglycerides $\geq 150 \mathrm{mg} / \mathrm{dl}$ or $\mathrm{HDL}$ cholesterol $\geq 40$ $\mathrm{mg} / \mathrm{dl}$, or BP $\geq 140 / 90 \mathrm{mmHg}$ or taking BP medication ${ }^{19}$. The two criteria could potentially lead to higher estimates of metabolic syndrome than IDF criteria. For instance, the WHO criteria utilize insulin resistance which, in pathogenesis term, precede raised fasting blood glucose used by the IDF definition, and while the NCEP definition uses waist circumference to measure obesity, the IDF utilizes the BMI.

The $1.7 \%$ prevalence of metabolic syndrome among normotensive women at one year is lower than the $24.3 \%$ reported from a non-pregnant women population in Nigeria in which the IDF definition was also used ${ }^{17}$. This disparity could be explained by the fact that the normotensive population in our study had fewer risk factors compared to women in this random survey of suburban population in whom $42 \%$ were overweight with mean systolic blood pressure of $131 \mathrm{mmHg}$ (112 $\mathrm{mmHg}$ in our normotensive cohort after one year of follow up). ${ }^{17}$

Because the cardiometabolic changes (reminiscent of metabolic syndrome) in HDP also occur in normal pregnancies and the effects could persist throughout puerperium and for variable length of postpartum period, we assessed metabolic syndrome from nine weeks postpartum up to one 
year - after which most pregnancy-related metabolic changes are expected to have waned off; and included normotensive women as a control group. The finding that only one new onset metabolic syndrome occurred out of fifty-nine normotensive observations at one year $(I R=$ 16.9/1000 person years) as opposed to sixteen out of 278 HDP observations (IR $=57.5 / 1000$ person years) - with incidence rate ratio of 3.4/1000 person years- further strengthened the association between metabolic syndrome with prior HDP.

We observed that at six months post-delivery of an HDP-complicated pregnancy, the likelihood of developing metabolic syndrome diminishes in the order of HDP sub-type severity: preeclampsia, chronic hypertension in pregnancy and gestational hypertension. Although preeclampsia has the highest risk at six months postpartum, the prevalence of metabolic syndrome among pre-eclamptic cohort was only 3.7\%. Other studies reported both higher and lower levels: a study in the Netherlands observed $13.9 \%$ prevalence at six months based on the WHO definition, ${ }^{13}$ and a Korean study reported no association with development of metabolic syndrome postpartum among women with pre-eclampsia who had two or more components of metabolic syndrome-pre-pregnancy. ${ }^{20}$ Although we had no information on the pre-pregnant status of our cohort, the prevalence of $17.4 \%$ metabolic syndrome at delivery may suggest a population with already pre-exiting abnormal metabolic components which might have conferred some protection against metabolic syndrome developing postpartum.

Only parity significantly exerts independent influence on occurrence of metabolic syndrome one year postpartum in our HDP cohort. By intuition, this should be expected as increasing parity is typically associated with advancing maternal age, hypertension, and abnormal BMI which, in turn, increases the risk of abnormal cardiovascular surrogate marker, including metabolic syndrome. Studies of Hispanic/Latino women in the United States ${ }^{21}$ and Chinese women ${ }^{22}$ have established significant association between higher parity and some components of metabolic syndrome independent of age. The American study observed that higher parity accompanied with abdominal obesity suggest high risk of metabolic dysregulation ${ }^{21}$. 


\section{Clinical and research implication}

West Africa in general, and Nigeria in particular, carry a heavier burden of CVDs risk compared to other regions ${ }^{23}$. It is projected to be the leading cause of premature mortality and disabilityadjusted life years (DALY) burden by $2050^{24}$. In addition, West Africa has one of the highest fertility rates with 5.2 children per woman (in contrast to 4.4 for Africa overall) ${ }^{23}$. Given the association between parity, HDP, metabolic syndrome and future risk of adverse cardiometabolic disorders, ${ }^{24}$ routine screening and management of metabolic syndrome following HDP - and especially when associated with multiparity - is essential, and should be included in any strategy to reduce the CVD burden in sub-Sahara Africa. Although screening for CVD risk markers following HDP-complicated pregnancies is an established practice in many high-income countries, there is no agreement on timing of commencement of screening and of ongoing follow up processes ${ }^{25}$. Guidelines for screening strategies for postpartum detection of metabolic syndrome following HDPs in high-income country settings are currently limited with only one template suggested by Dutch researchers which entails a stepwise approach starting at a younger age ${ }^{25}$. For the Nigerian, and possibly other (West) African health care contexts, commencement of screening during the pre-conceptional and antenatal periods would be recommendable, as the modification of risk for future CVDs based on body weight and blood pressure management, also reduces the risk of HDP in the shorter term. Importantly, up to $40 \%$ of our HDP cohort did not receive antenatal care. A general continued effort to promote (early) antenatal care attendance remains urgent. For pregnancies complicated by HDP, secondary prevention could commence within first year of delivery for a timely detection and management of metabolic syndrome in order to mitigate future risks of cardiovascular and metabolic disorders.

\section{Strengths and limitations}

This unique study prospectively followed over five hundred women with HDP and normotensive pregnancies in sub-Saharan Africa for one year and evaluated their health at three different time periods: nine weeks, six months and one year postpartum. It allowed the exploration of both prevalence and incidence rates and risk factors, including the comparison between women with hypertensive disorders and those with normal BP during pregnancy. However, some limitations 
need to be considered in the interpretation of these findings. First, that one year of follow up after delivery is not adequate to detect transition beyond the intermediary risk factors on the pathways to develop cardiovascular diseases to outcomes itself. Secondly, while we aimed for a minimum of 185 normotensive pregnancies, less than half were recruited. This was due to lower consent rates to participate in the study by women in that category ( $(95 \%$ and $38 \%$ for HDPs and normotensive respectively - due to low perception of risk and threat among the normotensive). This could have led to insufficient power to detect metabolic syndrome at different time intervals given the low(er) prevalence. At the same time, our sample size is sufficient to suggest that these will be at the very low ends and still justifies our conclusion to focus efforts on reducing the metabolic syndrome prevalence primarily among women with HDP at highest risk.

\section{Conclusion}

Hypertensive disorders in pregnancy are associated with increased risk of metabolic syndrome after delivery, and this is augmented by parity independent of age. Maternal BMI and blood pressure are the main drivers of metabolic syndrome in these women. Given the growing burden of cardiovascular disease on morbidity and mortality in sub-Saharan Africa, as well as availability of risk reducing strategies, screening of all women with HDP for metabolic syndrome should routinely take place starting within the first year of delivery (but also during pre-conceptional and antenatal periods).

\section{Funding}

This work was conducted as part of the Ending Eclampsia project (2014-2019), supported by the US Agency for International Development (USAID), under the terms of cooperative agreement number [USAID APSOAA-14000048]. This article reflects the views of the authors and does not represent the views of the U.S. Government. Salisu Ishaku was also supported by the UMC Utrecht Global Health Fellowship Program.

Conflict of interest

None

Authors Contribution

First and fifth authors made substantial contributions in the conception of the work. Second and third authors made the first draft. First, second, fifth and sixth authors revised critically for important intellectual contents. First 
and fourth authors conducted the data analysis while the remaining authors provide final revision and approval of the version to be published. 


\section{References}

1. Hernandez-Camacho JD, Hernandez-Camacho M. Clinical Update on metabolic syndrome Rev Esp Nutr Hum Diet 2017; 21(4): 384-392

2. The International Diabetes Federation consensus worldwide definition of the metabolic syndrome. Retrived from https://www.idf.org/e-library/consensus-statements/60idfconsensus-worldwide-definitionof-the-metabolic-syndrome.html on February 03 , 2020

3. Nijdam ME, Timmerman MR, Franx A. Cardiovascular risk factor assessment after preeclampsia in primary care. BMC Fam Pract 2009; 10:77.

4. Barry DR, Utzschneider KM, Tong J, et al. Intraabdominal fat, insulin sensitivity, and cardiovascular risk factors in postpartum women with a history of preeclampsia. Am J Obstet Gynecol 2015; 213:104. e1-11.

5. Jonsdottir L, Arngrimsson R, Geirsson R et al. Death rates from ischemic heart disease in women with a history of hypertension in pregnancy. Acta Obstet Gynecol Scand 1995; 74:772-6

6. Libby G, Murphy DJ, McEwan NF, et al. Preeclampsia and the later development of type 2 diabetes in mothers and their children: an intergenerational study from the Walker cohort. Diabetologia 2007; 50:523-30.

7. Carr DB, Newton KM, Utzschneider KM, et al. Preeclampsia and risk of developing subsequent diabetes. Hypertens Pregnancy 2009; 28:435-47

8. Guidelines for the Prevention of Stroke in Women: A Statement for Healthcare Professionals from the American Heart Association/American Stroke Association. Stroke. 2014; 45:1545-1588.

9. Erin PF, Emily JJ, Teri LH. Pregnancy Reveals Evolving Risk for Cardiometabolic Disease in Women. JOGNN, 45, 413-425; 2016. http://dx.doi.org/10.1016/j.jogn.2016.02.004

10. Jean-Claude F, Joe"I G, Jacques M et al. Early Occurrence of Metabolic Syndrome After Hypertension in Pregnancy. Obstetrics \& Gynecology; 2005:105(6). doi: 10.1097/01.AOG.0000163252. 02227.f8 
11. Magee LA, Dadelszen PV; Stones W et al. An Evidence-based guide to monitoring, prevention and management. The FIGO textbook of pregnancy hypertension: 2016: 5-6

12. National Institute for Health and Care excellence. Hypertension in pregnancy: diagnosis and management. Published: 25 June 2019, retrieved August 10, 2020 from www.nice.org.uk/guidance/ng133

13. Al-Nasiry S, Ghossein-Doha C, Polman SEJ et al. Metabolic syndrome after pregnancies complicated by pre-eclampsia or small-for-gestational-age: a retrospective cohort. BJOG 2015; 122:1818-1823.

14. Teixeira HACP, Costa CFH, Feitosa $\mathrm{HN}$ et al. Metabolic syndrome after pre-eclampsia: a cohort study with a mean follow up of 14 years. J Metabolic Syndrome 2013;3(3):1-7.

15. Udenze IC. Association of pre-eclampsia with metabolic syndrome and increased risk of cardiovascular disease in women: a systematic review. Niger J Clin Pract 2016; 19:431435

16. Turpin CA, Ahenkorah L, Owiredu WKBA et al. The Prevalence of the Metabolic Syndrome Among Ghanaian Pregnancy-Induced Hypertensive Patients Using the World Health Organisation and the National Cholesterol Education Program III Criteria. Journal of Medical Sciences 2008, 8: 443-451.

17. Ayokunle SD, Daisi DA, Peter OA et al. Metabolic Syndrome and Framingham Risk Score: Observation from Screening of Low-Income Semi-Urban African Women. Medicines 2016, 3, 15; doi:10.3390/medicines3020015

18. Oguoma VM, Nwose EU, Richards RS. Prevalence of cardio-metabolic syndrome in Nigeria: a systematic review. $p$ u b lich e a I th 2015;413-423

19. Huang PL. A comprehensive definition for metabolic syndrome. Dis Model Mech. 2009;2(5-6):231-237. doi:10.1242/dmm.001180

20. Geum JC, Un SJ, Jae YS et al. Is preeclampsia itself a risk factor for the development of metabolic syndrome in the postpartum period? Obstet Gynecol Sci 2019;62(4):233-241 
21. Catherine JV, Anna MS, Daniela $S$ et al. Parity and Components of the Metabolic Syndrome Among US Hispanic/Latina Women: Results From the Hispanic Community Health Study/Study of Latinos. Circ Cardiovasc Qual Outcomes (2016) Vol 9, No. 2_suppl_1. DOI: 10.1161/CIRCOUTCOMES.115.002464

22. Jing W, Guiqiang $X$, Lijun $S$ et al. Parity and risk of metabolic syndrome among Chinese women. Journal of women's health, 2015; Vol. 24, No. 7_ 602 - 607; DOI: 10.1089/jwh.2014.5134.

23. Gregory AR, Mark DH, Andrew EM et al. Global and Regional Patterns in Cardiovascular Mortality from 1990 to 2013. Circulation. 2015; 132:1667-1678. DOI: 10.1161/CIRCULATIONAHA.114.008720.)

24. Suzan H, Wim G, Milena P. Trends in cardiovascular diseases and associated risks in subSaharan Africa: a review of the evidence for Ghana, Nigeria, South Africa, Sudan and Tanzania, The Aging Male, 2019. 22:3, 169-176, DOI: 10.1080/13685538.2019.1582621

25. Katrien JG, Bas BVR, Arie F et al. Preventing cardiovascular disease after hypertensive disorders of pregnancy: Searching for the how and when. European Journal of Preventive Cardiology; 2017;. 24(16): 1735-1745 


\section{Chapter 6}

Prevalence and determinants of chronic kidney disease in women with hypertensive disorders in pregnancy in Nigeria: a cohort study

Salisu M. Ishaku

Timothy Olusegun Olanrewaju

Joyce L. Browne

Kerstin Klipstein-Grobusch

Kayode Gbenga

Arie Franx

Diederick E. Grobbee

Charlotte Warren

Submitted to Pregnancy Hypertension Journal, responded to reviewers' comments and awaiting decision 


\begin{abstract}
Background

Worldwide, hypertensive disorders in pregnancy (HDP) complicate between $5-10 \%$ of pregnancies. Sub-Saharan Africa is disproportionately affected by a high burden of HDP and chronic kidney disease (CKD). Despite mounting evidence associating HDP with the development of CKD, data from SSA are scarce.
\end{abstract}

\title{
Methods
}

Women with HDP $(n=410)$ and normotensive women $(n=78)$ were recruited at delivery and prospectively followed-up for one year. Serum creatinine was measured and the estimated glomerular filtration rates(eGFR) using CKD-Epidemiology equation determined. CKD was defined as decreased eGFR $<60 \mathrm{~mL} / \mathrm{min} / 1.73 \mathrm{~m} 2$ lasting for $\geq$ three months. Prevalence of CKD at six months and one year after delivery was estimated. Logistic regression analyses were conducted to evaluate predictors of CKD at six months and one year postpartum.

\section{Results}

Within 24 hours of delivery, nine weeks and six months postpartum, women with HDP were more likely to have a decreased eGFR compared to normotensive women (12\%, 5.7\%, 4.3\% versus $0 \%$, $2 \%$ and $2.4 \%$, respectively). The prevalence of CKD in HDP at six months and one year postpartum was $6.1 \%$ and $7.6 \%$, respectively, as opposed to zero prevalence in the normotensive women for the corresponding periods. Proportions of decreased eGFR varied with HDP sub-types and intervening postpartum time since delivery, with pre-eclampsia and eclampsia showing higher prevalence than chronic and gestational hypertension. Only maternal age independently predicted occurrence of decreased eGFR at six months postpartum $(\mathrm{aOR}=1.18 /$ year; $95 \% \mathrm{Cl} 1.04-$ 1.34). 


\section{Conclusion}

Prior HDP was associated with risk of future CKD, with prior HDP being more likely to experience evidence of CKD over periods of postpartum follow-up. Routine screening of women following HDP-complicated pregnancies should be part of a postpartum monitoring program to identify women at higher risk. Future research should report on both the eGFR and total urinary albumin excretion to enable detection of women at risk of future deterioration of renal function.

Key words: Hypertensive disorder in pregnancy, chronic kidney disease, Nigeria, low- and middleincome countries, sub-Saharan Africa 


\section{Introduction}

Hypertensive disorders in pregnancy (HDP) complicate $5-10 \%$ of pregnancies world-wide $\mathrm{e}^{1,2,8}$ and are annually responsible for more than 500,000 and 70,000 global neonatal and maternal deaths respectively ${ }^{2}$. HDP include chronic hypertension, gestational hypertension and (pre-) eclampsia $^{1,2}$. Besides their contribution to immediate pregnancy-related complications, HDP are associated with long-term health consequences for both the mothers (for examples, cardiovascular diseases, type II diabetes, kidney diseases and mental health problems) and their babies (prematurity and low birth weight) ${ }^{1,2}$.

The association between kidney disease and HDP (especially pre-eclampsia) ${ }^{5}$ could be complex due to possibility of reverse causality. On one hand, previously undiagnosed pre-existing renal impairment can be unmasked by pregnancy (manifesting as hypertension and or proteinuria) ${ }^{4}$ due to increased demand on renal function, leading to wrong diagnosis of HDP ${ }^{3}$. On the other hand, renal function abnormalities can occur de novo in association with HDP, especially preeclampsia ${ }^{5}$. In both situations, the renal impairments may persist, and could lead to chronic kidney diseases (CKD) ${ }^{5}$. In addition, pre-eclampsia affects renal morphology ${ }^{6}$, leads to persistent microalbuminuria-9, and progression of CKD to end-stage kidney disease (ESKD) ${ }^{10-13}$. The associations between pre-eclampsia and CKD were shown to be stronger within 5 years of index pregnancies and weaker thereafter ${ }^{6,11}$.

Kidney disease is defined as "an abnormality of kidney structure or function with implications for the health of an individual, which can occur abruptly, and may either resolve or become chronic" $^{\prime 15}$. When kidney disease is present for $\geq 3$ months, it is described as chronic kidney disease (CKD)"15. In 2017, CKD accounted for 1.2 million and 35.8 million, respectively, of global deaths and disability adjusted life years (DALYs), with sub-Saharan Africa experiencing heavier burden for its state of development ${ }^{16}$. In 2017, 13,740 deaths occurred from kidney disease in Nigeria ${ }^{16}$. The contribution of HDP to the burden of CKD is not fully elucidated in sub-Saharan Africa in general and Nigeria in particular. Scarce data from a cross sectional study undertaken during pregnancy in Nigeria showed an association between pre-eclampsia and markers of kidney 
dysfunction ${ }^{18}$ and a small short-term follow up study of Cameroonian women with severe preeclampsia revealed persistent proteinuria of $31.5 \%$ and $1.8 \%$ at three and six months postpartum, respectively ${ }^{17}$. To date, no study from sub-Saharan Africa prospectively evaluated prevalence of CKD following deliveries of pregnancies complicated by hypertensive disorders. In this study, we examined the prevalence and determinants of CKD among women with hypertensive disorders in pregnancy over a 1-year period after delivery, at tertiary care settings in Nigeria.

\section{Methodology}

\section{Study design}

The study was a prospective cohort study. Women with HDP and women with normotensive pregnancies who delivered in the participating hospitals were recruited from August 2017 to April 2018 and followed up over a subsequent period of one year. The last woman recruited exited the study on March 31, 2019 after one year of follow up.

\section{Study setting}

The study was conducted at eight tertiary hospitals in the six geo-political zones of Nigeria. The hospitals were purposefully selected to reflect diversity in the country in terms of ethnic differences and socio-economic status. The following states (and hospitals) participated: Bauchi State (Abubakar Tafawa Balewa University Teaching Hospital, ATBUTH), Cross River State (University of Calabar Teaching Hospital, UCTH), Ebonyi State (Federal Teaching Hospital Abakaliki, FTHA), Kogi State (Federal Medical Center, FMC Lokoja), Kano State (Aminu Kano Teaching Hospital, AKTH), Ondo State (Mother and Child Hospital Akure and University of Medical Sciences Teaching Hospital, Ondo) and Sokoto State (Usmanu Danfodiyo University Teaching Hospital, UDUS). The facilities were high-volume sites with well-functioning antepartum, intrapartum, and postpartum clinics, delivery rooms, and laboratory services with combined average annual deliveries of 38,400 . 


\section{Participants}

Receiving delivery care services at the facilities, being 18 years or above, and had a diagnosis of HDP were the main eligibility criteria for inclusion in the study. The unmatched normotensive women were recruited from the same hospitals. HDP and normotensive women were recruited from similar population of women giving births in these facilities. Recruitment proceeded independently at all facilities. Case identification was carried out by specifically trained and experienced midwives using standard diagnostic criteria based on HDP definitions described above. Women with the following conditions (based on previously documented clinical history and diagnoses) were excluded: having multiple pregnancies, diabetes mellitus, sickle cell disease, heart disease, preexisting kidney disease and connective tissues disorders.

\section{Study procedures}

Normotensive women and women with HDP were informed of the study either during antenatal care or after delivery. All recruitments were done in the postpartum period. Those willing to participate were individually counseled and signed or thumb-printed informed consent form obtained (consent rate over 95\% among HDP, 35\% among normotensive). Enrollment forms, collecting information on socio-demographic and obstetrics variables such as age, body mass index (BMI), parity and booking status, were completed within 24 hours of delivery. After enrollment, the women underwent general and systemic clinical examination. In addition, laboratory investigations, including urine protein analysis and renal function tests, were performed on the participants before they were discharged from the hospitals. They were subsequently followed up undergoing the same clinical and laboratory investigations conducted at baseline, at nine weeks, six months and one year postpartum. To improve follow up rate, the research participants were requested to provide their contact information and spousal mobile telephone numbers. They were reminded of their follow up appointments through phone calls. Participants' contact information was not linked to their clinical records while all clinical information was linked to unique identifiers. 


\section{Exposure variables}

The main exposures of interest were the presence of any of the HDP sub-type including chronic hypertension, gestational hypertension and pre-eclampsia as defined by the ISSHP1,2. Hypertension was defined as systolic blood pressure of $\geq 140 \mathrm{mmHg}$ and or diastolic blood pressure of $\geq 90 \mathrm{mmHg}$ measured on two consecutive periods 4-6 hours apart. Chronic hypertension in pregnancy was defined as any hypertension with onset before the index pregnancy or diagnosed within the first 20 weeks of the index pregnancy. Gestational hypertension was defined as any hypertension occurring after the first 20 weeks of pregnancy without significant proteinuria (<2++ of proteinuria on urine dipstick measurement) or any hematological or biochemical abnormality. Pre-eclampsia was defined as hypertension with onset after the first 20 weeks of pregnancy with significant proteinuria $(\geq 2++$ of proteinuria on urine dipstick measurement) or the presence of any hematological and biochemical abnormality ${ }^{1,2}$.

\section{Outcome variables}

We assessed prevalence of CKD based on serum creatinine (Modified Jaffe Kinetic Method Roche C311 and Abbott C4000) and estimated glomerular filtration rate (eGFR) by CKDEpidemiology equation as recommended by the Kidney Disease Improving Global Outcomes for black women ${ }^{14}$ :

- $\quad$ eGFR for creatinine $\leq 0.7 \mathrm{mg} / \mathrm{dl}: 144 *$ (serum creatinine/0.7)-0.239*0.993Age *1.159]

- eGFR for creatinine $>0.7 \mathrm{mg} / \mathrm{dl}: 144^{*}$ (serum creatinine/0.7)-1.209* $\left.0.993^{\text {Age }} * 1.159\right]$

The CKD-epidemiology equation was used together with the GFR calculator of the National Kidney Foundation available at https://www.kidney.org/professionals/kdogi/gfr calculator. When the eGFR was $<60 \mathrm{~mL} / \mathrm{min} / 1.73 \mathrm{~m}^{2}$, it is considered decreased and when decreased eGFR persists for $\geq$ three months, it indicates presence of $C K D^{6,16}$.

\section{Data source/data collection}

At baseline-within 24 hours of delivery, 9 weeks, 6 months and one year postpartum, laboratory tests were performed on the women. We collected blood samples for serum urea and creatinine 
measurement and estimated glomerular filtration rate. Clinical examination, blood and urine sample collection were performed by trained medical officers who served as research assistants for this study. Laboratory tests were performed using techniques as reported above. Both the medical officers and the laboratory scientists were not aware of clients' categorization as either HDP or normotensive.

\section{Sample size}

Based on previous evidence that about $14 \%$ of women with pre-eclampsia had abnormal GFR, at least, four months after delivery ${ }^{17}$, we estimated that 185 women would be required to participate in the study, each for HDP and normotensive (at $5 \%$ alpha level and power of $80 \%$ ). Considering $10 \%$ potential loss to follow-up, 204 women were required to be recruited in each arm. However, we planned to enroll as many women as resources could accommodate given that the study was observational with minimal risks to the women. Because of the differential consenting rates between the two cohorts (95\% and 38\% for HDP and normotensive respectively - due to low perception of risk and threat among the normotensive), we were able to recruit 410 and 78 women with HDP and normotensive pregnancies respectively.

\section{Data management and statistical analysis}

The results of medical and laboratory investigations were entered into electronic data capturing platform (ODK) by trained research assistants. All women with HDP and normotensive women who were recruited and successfully followed-up for each period were analyzed using SPSS IBM version 25.0. Frequencies, percentages, proportion and means (standard deviations) were used to describe participants' baseline characteristics. For comparison of mean eGFR between normotensive women and women with HDP, independent t-tests was used in case of normally distributed data, otherwise the Mann-Whitney $U$ test was applied. To compare mean differences in eGFR between HDP sub-types (gestational hypertension, pre-eclampsia and eclampsia), oneway analysis of variance (ANOVA) was performed. A Tukey post hoc test was used to determine where the difference lied between the HDP sub-types in their mean eGFR. Univariable and multivariable logistic regression analyses were performed to assess predictors of CKD at six months and one year postpartum. Confounders' selection was theory-driven, and the following 
variables were included: age, BMI, parity, gestational age at delivery, early- and late-onset HDP, booking status and HDP sub-types.

Loss to follow up

Cases of loss to follow up were nearly similar between HDP and normotensive women and occurred when subjects failed to report for data collection for each period. Of the 410 women with HDP enrolled, $147(36 \%), 178(43 \%)$ and $132(32 \%)$ were lost to follow up at nine weeks, six months and one year postpartum, respectively. The corresponding values for the normotensive women were $25(32 \%), 35(45 \%)$ and $19(24 \%)$ respectively. We assumed data were missing completely at random. Therefore, complete case analysis was performed such that for any data collection period only clients that have reported and provided complete information were analyzed.

\section{Ethical approval}

The study was approved by the Population Council's institutional review board in New York (protocol no. 810), National Health Research Ethics Committee (NHREC) at the Federal Ministry of Health of Nigeria and by the institutional review boards at all the participating hospitals.

Results

Table I describes the baseline characteristics of women with HDP and those with normotensive pregnancies. A total of 410 women with HDP and 78 with normotensive pregnancies were enrolled. Women with HDP and normotensive were not significantly different in respect to their mean age $[29.5(S d=13.8)$ versus 28.3(Sd=5.1), p=0.48] and BMI [ 28.9(Sd=7.7) versus $26.8(S d=6.4), p=0.05$ ]. The proportion of multiparity ( $\geq 4$ previous deliveries) was similar in the two groups (24\%). Similarly, there was no significant difference in gestational age at booking [with mean booking gestational age of 23.7 weeks $(S d=6.3)$ and 22.8 weeks $(S d=6.4), p=0.301$ ] for women with HDPs and normotensive, respectively. Of women with HDP, about two-thirds (62.3\%) were late-onset ( $\geq 34$ weeks of gestation). However, based on HDP sub-types, late-onset disease occurred in $85 \%, 74 \%$ and $61 \%$ of women with gestational hypertension, eclampsia, and pre-eclampsia respectively. The prevalence of CKD in HDP at six months and one year postpartum 
was $6.1 \%$ and $7.6 \%$, respectively, as opposed to zero prevalence in the normotensive cohort for the corresponding periods. 


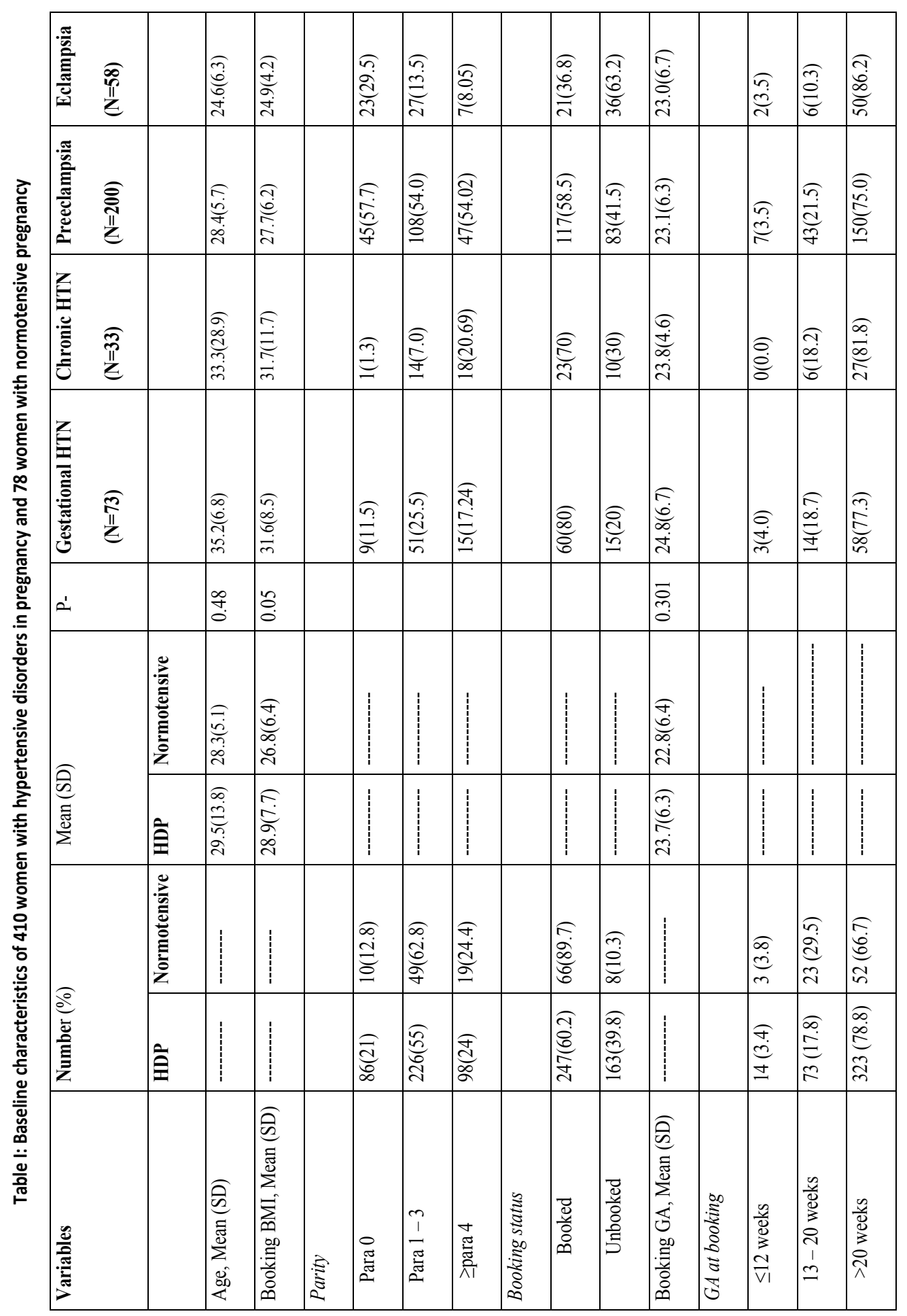




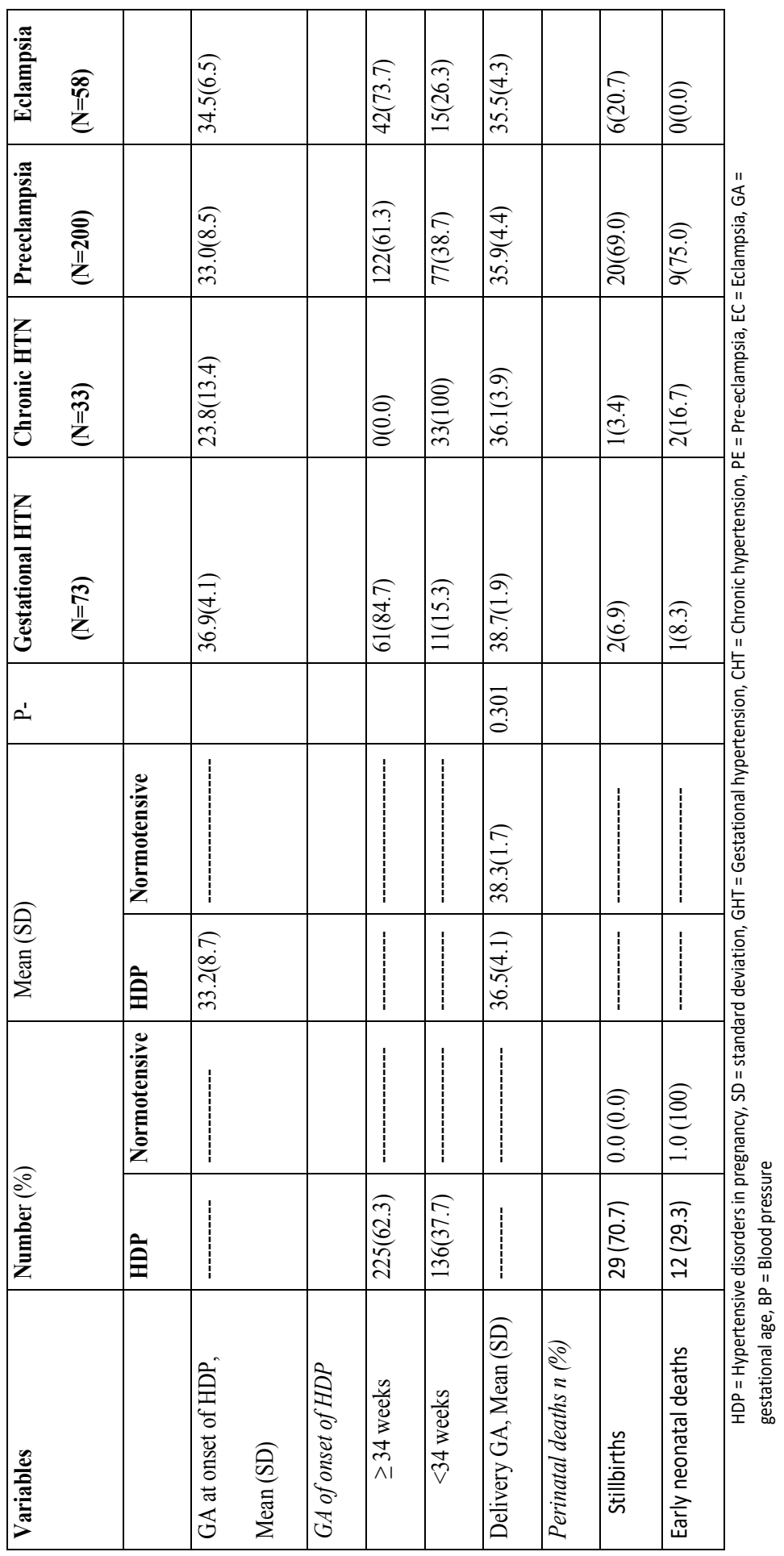




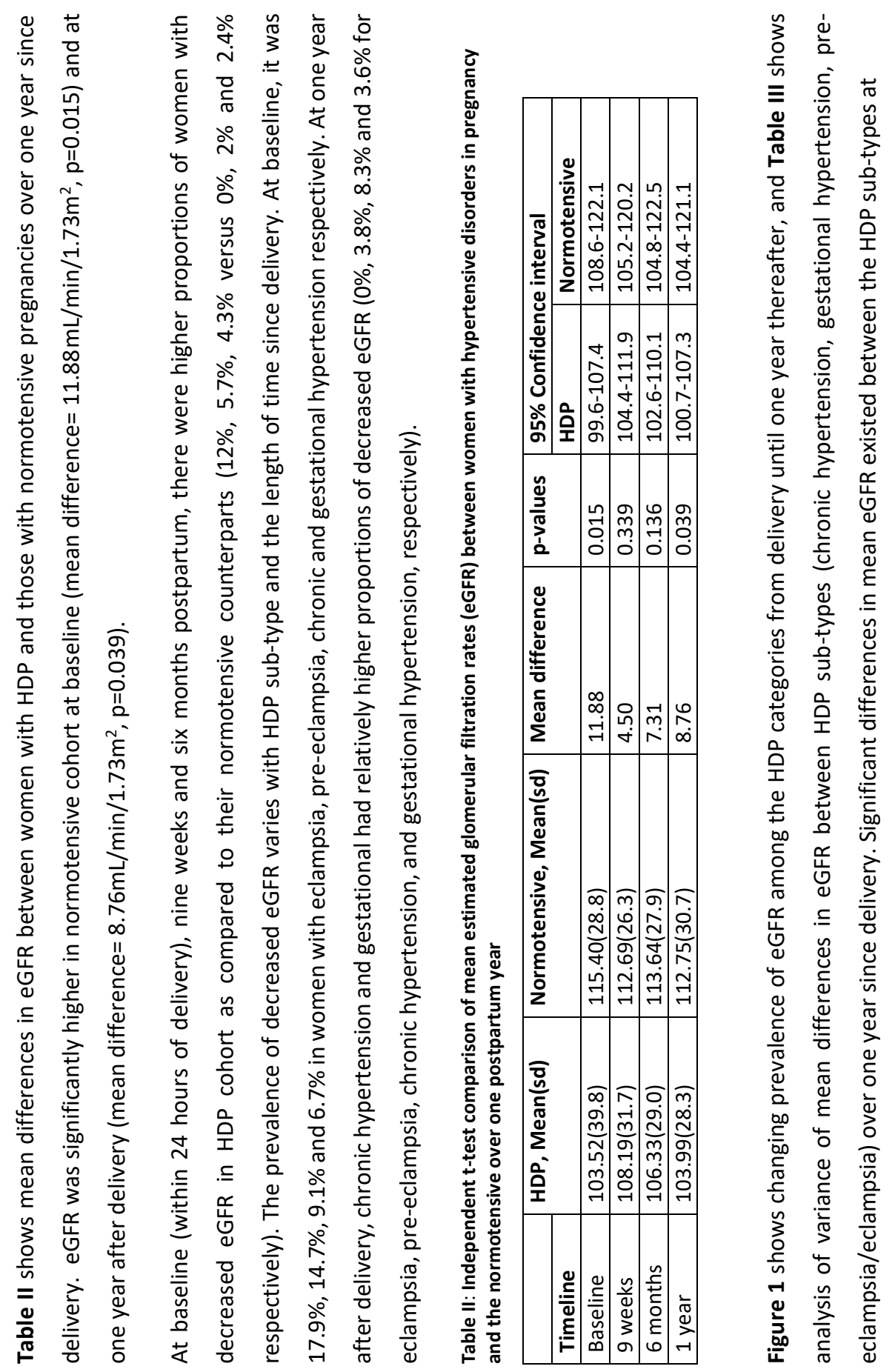


baseline $(p=0.0015)$ and at six months after delivery $(p=0.0194)$. Based on post-hoc test, the difference at baseline was between women with eclampsia and gestational hypertension $(p=0.001)$ while at six months after delivery, the difference was between pre-eclampsia and chronic hypertension $(p=0.014)$.

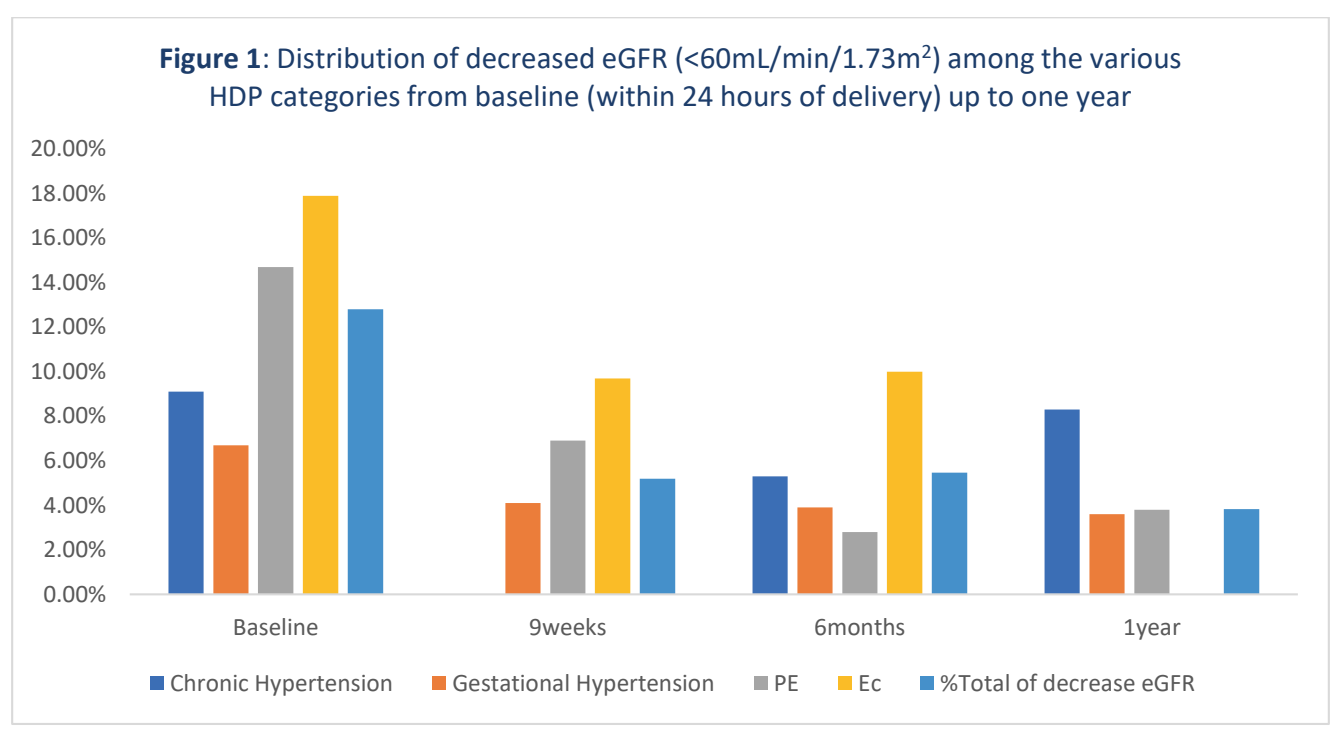




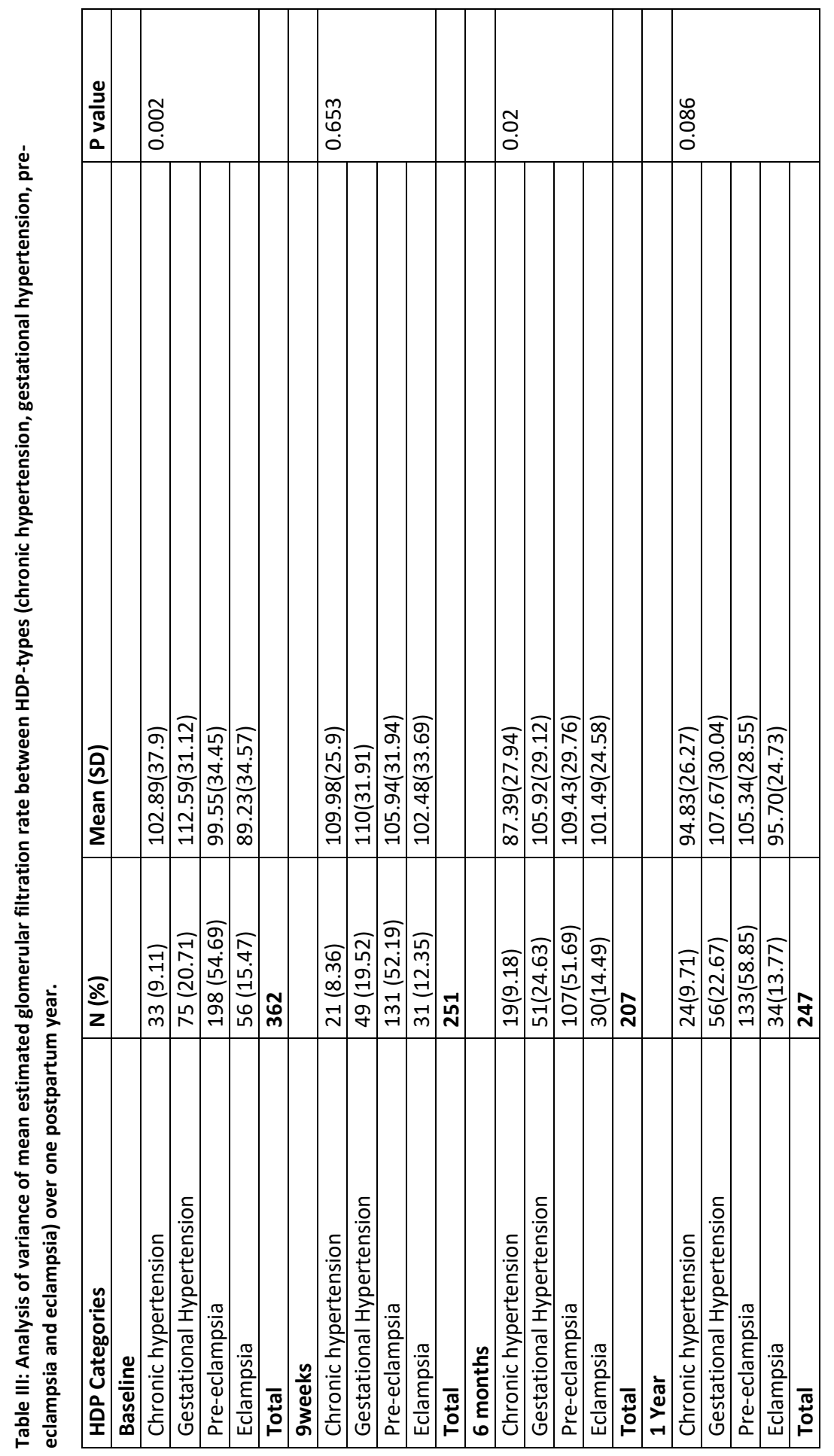


At one year postpartum, while the proportion of women with decreased eGFR was higher in the normotensive women than in the HDP cohort (5.5\% and 3.5\% respectively), the mean creatinine value was higher in the HDP cohort [(945umol/L(Sd=570) versus $844 \mathrm{umol} / \mathrm{L}(\mathrm{Sd}=270)]$.

Finally, Table IV describes the results univariable and multivariable logistic regression analysis of predictors of decreased eGFR in women with HDP at six months and one year after delivery. Only maternal age independently predicted the occurrence of decreased eGFR at six months postpartum ( $\mathrm{aOR}=1.18 /$ year; $95 \% \mathrm{Cl}=1.03-1.36)$. At one year after delivery, none of the included variables was a significant predictor. 


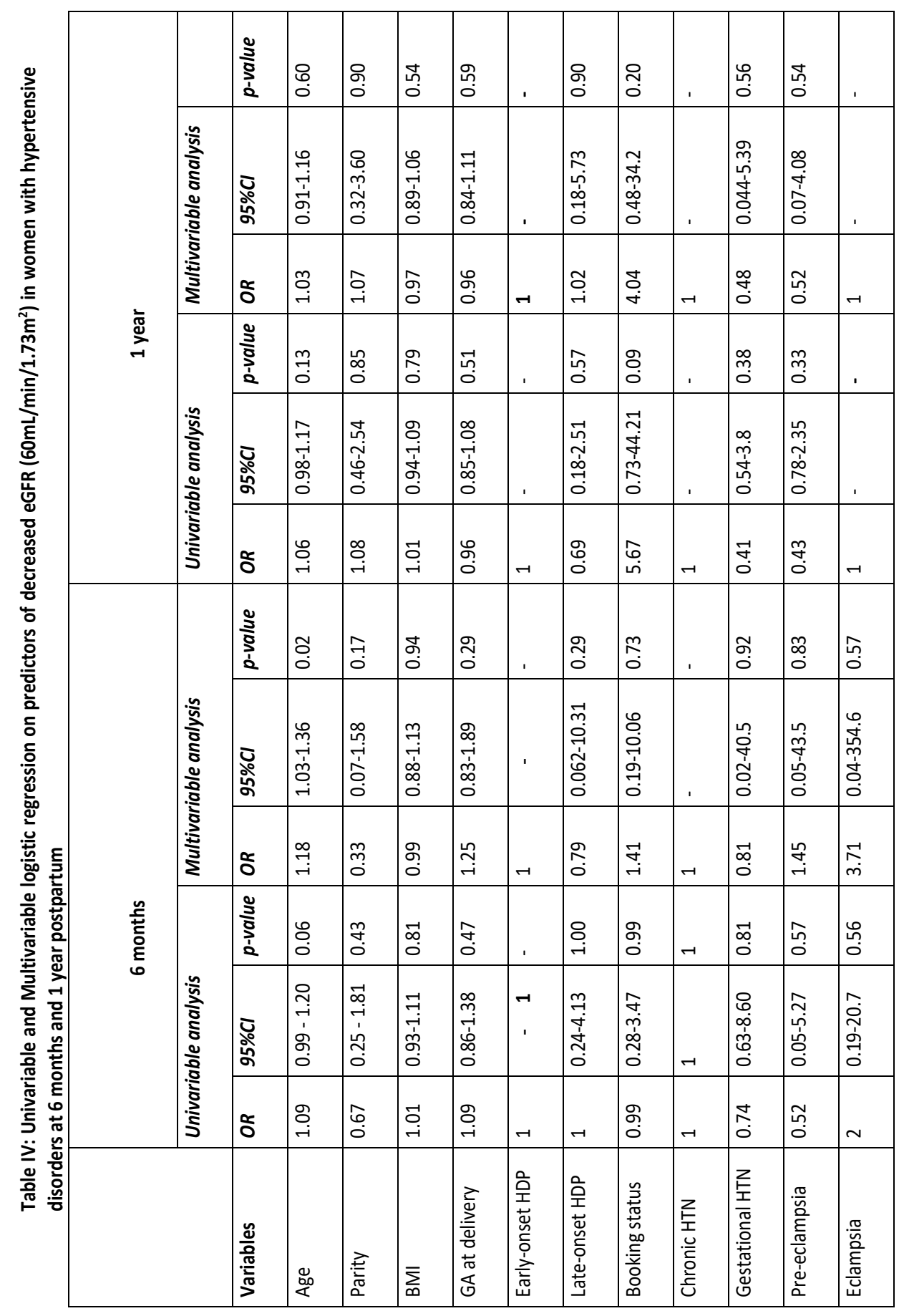




\section{Discussion}

The 2012 Kidney diseases: Improving Global Outcomes (KDIGO) provides guiding criteria for identification of chronic kidney disease which include either determining the presence of one or more markers of kidney disease or identifying presence of decreased estimated GFR (eGFR) $\left(<60 \mathrm{~mL} / \mathrm{min} / 1.73 \mathrm{~m}^{2}\right)$ lasting $\geq$ three months ${ }^{14-15}$. We used eGFR derived from serum creatinine (eGFR creat $_{\text {) }}$ to assess CKD in this study because it is the recommended approach ${ }^{14}$. We, therefore, reported prevalence of both the decreased eGFR and CKD. The findings that, at delivery, none of the normotensive women had decreased eGFR as opposed to $12 \%$ prevalence among the HDP is suggestive of adverse effect that HDP could have on renal function, with pre-eclampsia or eclampsia having more profound renal effect than chronic or gestational hypertension. To the best knowledge of the authors, no previous study reported eGFR at delivery following HDP.

Based on KDIGO guideline (2012), 7.6\% of HDP women with CKD at one year postpartum in our study will require yearly monitoring of their renal function ${ }^{14}$, as opposed to $13.7 \%$ reported for Dutch women ${ }^{19}$. We expect the prevalence to be higher in the Dutch study because the measurement was exclusively performed among women with pre-eclampsia who have the highest risk ${ }^{10-13}$. This is comparable to our pre-eclampsia participants with decreased eGFR at six months after delivery $(13.7 \% \text { versus } 12.8 \% \text { respectively })^{19}$. It is not unexpected that preeclampsia/eclampsia are associated with more profound renal impairment than the other HDP sub-types as they result in glomerulo-endotheliosis which affects renal parenchyma and functions, an effect absence in chronic and gestational hypertension ${ }^{23-25}$. However, a Taiwanese study reported that previous history of any HDP type has greater than 9 folds risk of leading to future $\mathrm{CKD}\left(\mathrm{HR}=9.38(95 \% \mathrm{Cl} 7.09-12.4)^{10}\right.$. A long-term Scottish cohort study revealed prevalence of CKD of $7.5 \%$ and $5.2 \%$ in women who previously had gestational hypertension and pre-eclampsia, respectively ${ }^{26}$.

As pregnancy-related effect of HDP on kidney function wanes in the postpartum period, the proportions of women with decreased eGFR among the HDP declined progressively up to six months. However, at every postpartum time, mean eGFR is lower in HDP than in their 
normotensive counterparts. This pattern was also observed by Paauw et al. in Groningen, although this was from a historical cohort after several years since delivery ${ }^{21}$. But at the same time, proportions of decreased eGFR rose progressively (albeit marginally) among the normotensive women. It could mean that while HDP adversely affects renal function in pregnancy resulting in decreased eGFR, women who remain normotensive during pregnancy may not show renal function derangement until after delivery perhaps in those with pre-existing renal impairments. This suggestion requires further investigation.

HDP categories do not seem to affect kidney function to the same degrees, and their differential impact seemed to vary with increasing length of postpartum follow up time. Immediately after delivery and up to the end of puerperium, kidney function was better in descending order of gestational hypertension, chronic hypertension, pre-eclampsia, and eclampsia based on the eGFR. After six postpartum months onward, women with chronic and gestational hypertension in pregnancy fared the worst, although pre-eclampsia continued to be associated with decreased eGFR. While it is understood that pre-eclampsia affects kidney function more than the other HDP sub-types, why women with gestational/chronic hypertension had better eGFR than preeclampsia/eclampsia in first 6 months of delivery with reversing trend thereafter is not very clear, and has not been reported previously.

Given the strong association between maternal age and CKD among our HDP cohort, the importance of maternal age in the management of HDP becomes necessary. End-stage renal disease occurred in Norwegian women with previous pre-eclampsia at mean age of 41 years 22 . Women in sub-Saharan Africa have relatively wider reproductive experience (commencing childbirths much earlier and stopping late) than elsewhere, with mean age of women seeking to limit pregnancy and childbirth being 37 years $^{20}$. In this study, women as old as 57 were still giving birth. This implies that women in the sub-region could suffer double jeopardy in respect to HDP on account of their relatively advanced maternal age; have higher predisposition to HDP which increases the likelihood of future chronic health conditions including chronic kidney diseases. 


\section{Study strength and limitations}

Globally, this is among the first studies to estimate the prevalence of chronic kidney disease based on estimated GFR as recommended by the KDIGO 2012, and to the best of authors' knowledge the first in sub-Saharan Africa especially in women with previous history of HDP. Because our cohort was recruited and assessed at delivery and prospectively followed for one year, we were able to report prevalence of decreased eGFR within 24 hours after childbirth. This information is lacking in previous studies. Our study is not without limitations. While we aimed to recruit a minimum of 185 normotensive pregnancies, we only recruited less than half as a results of lower consent rates by women with normal pregnancies. This could have led to insufficient power to detect presence of decreased eGFR and or CKD as appropriate among the normotensive cohort. On the other hand, our sample size is sufficient to suggest that these will be at the very low ends and still justifies our conclusion to focus efforts on reducing CKD prevalence primarily among women with HDP who are at highest risk.

Although our study was considerably affected by high number of missed appointments at various follow-up periods, the proportions of missed appointments were nearly similar at all time periods between women with HDP and those with normotensive pregnancies. Because our assumption to data missing completely at random may not hold true, the complete case analysis performed could have led to erroneous estimates. However, analyses of loss to, versus complete follow up at one year for both the HDP and the normotensive did not show significant difference in most of the demographic and clinical parameters between the two groups (see supplement table I \& II). Only among the HDP cohort that serum creatinine and cholesterol were significantly different with loss to follow up having higher mean creatinine values at baseline. This means that the prevalence of CKD reported in this article could have been higher were the loss to follow ups accounted for. Serum cholesterol is not known to affect renal functions.

We were unable to report on the risk of deteriorating kidney function because to do this requires estimating both the GFR and a 24-hour urinary albumin excretion which we could not do under this research setting. Although some investigators reported that risk of kidney diseases following HDP becomes weaker after 5 years of delivery ${ }^{6,11}$, others reported an average of 17 years 
postpartum for kidney disease occurrence after pre-eclampsia in first pregnancy ${ }^{22}$. We were unable to follow our cohorts for these durations which limits our ability to comment on longterm association between HDP and CKD. Finally, we have no information on women's pre-existing medical conditions which might have confounded our observations and interpretation by ascribing renal impairment to prior HDP in women with undocumented pre-existing renal disease before pregnancy, as reported by other researchers ${ }^{9}$.

\section{Conclusion}

Due of high fertility rate in sub-Saharan Africa, HDP will continue to place a high burden on women's health during their reproductive years and beyond. With the mounting evidence suggesting an association between prior HDP and risk of future CKD, routine screening of women following HDP-complicated pregnancies should be part of our postpartum workup to identify women in need of close monitoring. In addition, future studies should report both the eGFR and urinary albumin excretion to detect women at risk of deteriorating renal function.

\section{Acknowledgement}

We wish to acknowledge the contribution of Titia Lely who provided guide and technical support to some methodological aspects in this article

\section{Funding}

This work was conducted as part of the Ending Eclampsia project (2014-2019), supported by the US Agency for International Development (USAID), under the terms of cooperative agreement number [USAID APSOAA-14000048]. This article reflects the views of the authors and does not represent the views of the U.S. Government. Salisu Ishaku was also supported by the UMC Utrecht Global Health Fellowship Program.

\section{Authors Contribution}

First and third authors made substantial contributions in the conception of the work. First author made the first draft. Second, third, fourth and fifth authors revised critically for important methodological and intellectual contents. First author conducted the data analysis while the remaining authors provide final revision and approval of the version to be published. 
Supplement I: Comparative analyses of characteristics of completed versus loss to follow up at one year among women with hypertensive disorders in pregnancy and the normotensive counterpart.

\begin{tabular}{|l|l|l|l|l}
\hline & \multicolumn{2}{|c|}{ HDP } & P Value & Normotensive \\
\hline Variables & Followed up n(mean) & Loss to follow up n(mean) & & Followed up n(mean) \\
\hline Mean age & $278(29.8)$ & $132(29.1)$ & 0.641 & $58(28.2)$ \\
\hline $\begin{array}{l}\text { Mean Gestational age } \\
\text { at onset of HDP (SD) }\end{array}$ & $245(33)$ & $120(33.5)$ & 0.442 & \\
\hline $\begin{array}{l}\text { Mean Gestational age } \\
\text { at booking (SD) }\end{array}$ & $174(23.4)$ & $71(24.3)$ & 0.040 & $54(22.9)$ \\
\hline Mean BMI at baseline & $271(29.2)$ & $121(28.2)$ & 0.958 & $58(28.2)$ \\
\hline $\begin{array}{l}\text { Mean systolic BP at } \\
\text { baseline (mmHg) }\end{array}$ & $219(158.8)$ & $43(161.7)$ & 0.464 & $46(111.2)$ \\
\hline $\begin{array}{l}\text { Mean diastolic BP at } \\
\text { baseline (mm/Hg) }\end{array}$ & $219(137.1)$ & $43(135)$ & 0.786 & $46(73.2)$ \\
\hline $\begin{array}{l}\text { Mean creatinine at } \\
\text { baseline (mg/dL) }\end{array}$ & $278(0.949)$ & $126(1.191)$ & 0.014 & $58(0.848)$ \\
\hline Cholesterol (mmol/l) & $277(4.529)$ & $127(4.052)$ & 0.008 & $58(4.296)$ \\
\hline Triglycerides (mmol/l) & $276(1.798)$ & $122(1.823)$ & 0.819 & $54(1.631)$ \\
\hline
\end{tabular}

SUPPLEMENT II: Distribution of Sociodemographic and Obstetric Characteristic of completed versus lost to follow up among the HDP at one year

\begin{tabular}{|c|c|c|}
\hline Variables & Followed up to 1 year & Loss to follow up at one year \\
\hline Mean age (SD) & $29.8(6.2)$ & $29.1(19.9)$ \\
\hline Mean Gestational age at onset of HDP (SD) & $33(9.9)$ & $33.5(7.3)$ \\
\hline Mean Gestational age at booking (SD) & $23.4(6.8)$ & $24.3(5.6)$ \\
\hline \multicolumn{3}{|l|}{ Category of HDP } \\
\hline Chronic Hypertension n (\%) & $15(7.54)$ & $18(11.1)$ \\
\hline Gestational hypertension $\mathrm{n}(\%)$ & $52(26.1)$ & $20(12.4)$ \\
\hline Pre-eclampsia n (\%) & $107(53.8)$ & $92(56.8)$ \\
\hline Eclampsia $\mathrm{n}(\%)$ & $25(12.6)$ & $32(19.8)$ \\
\hline Mean BMI at baseline (SD) & $29.2(7.9)$ & $28.2(7.5)$ \\
\hline Mean BMI at 1 year (SD) & $28.7(7.2)$ & \\
\hline Mean systolic BP at baseline (SD) & $158.8(20.8)$ & $161.7(23.3)$ \\
\hline Mean systolic BP at 1 year (SD) & $133.3(26.6)$ & 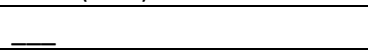 \\
\hline Mean diastolic at baseline (SD) & $137.1(23.4)$ & $135(29.3)$ \\
\hline Mean diastolic at 1 year (SD) & $86.1(18.3)$ & - \\
\hline
\end{tabular}




\section{References}

1) Laura AM, Peter VD, William S., Mathew M. (2016). The FIGO textbook of pregnancy hypertension: An Evidence-based guide to monitoring, prevention and management. ISBN: 978-0-9927545-5-6

2) Mark AB, Laura AM, Louise CK et al. Hypertensive Disorders of Pregnancy ISSHP Classification, Diagnosis, and Management Recommendations for International Practice. Hypertension. 2018; 72:24-43. DOI: 10.1161/HYPERTENSIONAHA.117.10803.

3) Maria LGS, Andrea K, Joseph PG, Vesna G. Renal Disorders in Pregnancy: Core Curriculum 2019. Am J Kidney Dis (2018). 73(1): 119-130.

4) Brown MA, Magee LA, Kenny LC, et al, International Society for the Study of Hypertension in Pregnancy (ISSHP). The hypertensive disorders of pregnancy: ISSHP classification, diagnosis \& management recommendations for international practice. Pregnancy Hypertens 2018; 13:291310. doi: 10.1016/j.preghy.2018.05.004

5) Jonas HK, Saima B, Jan W, Mette BD, Heather AB. Pre-eclampsia and risk of later kidney disease: nationwide cohort study. British Medical Journal 2019;365: I1516

6) Gaber LW, Spargo BH, Lindheimer MD. Renal pathology in pre-eclampsia. Baillieres Clin Obstet Gynaecol 1994; 8:443-68

7) Wieteke MH, Ralph RS, Fred KL, Marc EAS. History of preeclampsia is more predictive of cardiometabolic and cardiovascular risk factors than obesity. European Journal of Obstetrics \& Gynecology and Reproductive Biology 194 (2015) 189-193

8) Bar J, Kaplan B, Wittenberg C, et al. Microalbuminuria after pregnancy complicated by preeclampsia. Nephrol Dial Transplant 1999; 14:1129-32.

9) Andrea GK, Vesna DG. From Delivery to Dialysis: Does Preeclampsia Count? Am J of Kidney Dis(2018). Vol 71; 601 - 604. doi: 10.1053/j.ajkd.2018.02.005

10) I-Kuan W, Chih-Hsin M, Yi-Chih C et al. Association between hypertensive disorders during pregnancy and end-stage renal disease: a population-based study. Canadian Medical Association Journal, February 19, 2013, 185(3)

11) McDonald SD, Han Z, Walsh MW, et al. Kidney disease after preeclampsia: a systematic review and meta-analysis. Am J Kidney Dis2010; 55:1026-39

12) Vikse BE, Irgens LM, Leivestad T, Skjaerven R, Iversen BM. Preeclampsia and the risk of endstage renal disease. New England Journal of Medicine. 2008;359(8):800-809. 
13) Kattah AG, Scantlebury DC, Agarwal S, et al. Preeclampsia and ESRD: the role of shared risk factors. Am J Kidney Dis.2016;69(4):498-505.

14) KDIGO 2012 clinical practice guideline for the evaluation and management of chronic kidney disease. Kidney international; Volume 3 Issue 1

15) Adeera L, Paul ES. Summary of KDIGO 2012 CKD Guideline: behind the scenes, need for guidance, and a framework for moving forward. Kidney International (2013) 85, 49-61; doi:10.1038/ki.2013.444

16) Boris B, Carrie P, Andrew SL et al. Global, regional, and national burden of chronic kidney disease, 1990-2017: a systematic analysis for the Global Burden of Disease Study 2017. Lancet 2020; 395: 709-33.

17) Kaze, FF, Francis AN, Andre-Pascal K et al. Postpartum trend in blood pressure levels, renal function and proteinuria in women with severe pre-eclampsia and eclampsia in sub-Saharan Africa: A 6-month cohort study. BMC Pregnancy and Childbirth 2014 14:134.

18) Oloruntoba $A E$, Oluwatumininu MO, Christian CM, Nkeiruka OO. Biochemical Assessment of Renal and Liver Function among Preeclamptics in Lagos Metropolis. International Journal of Reproductive Medicine (2018). https://doi.org/10.1155/2018/1594182

19) Veronica ALB, Julia JS, TC, Marc EAS. Prevalence of chronic kidney disease after preeclampsia. J Nephrol (2017) 30:403-409; DOI 10.1007/s40620-016-0342-1

20) Van LLM, Yahner M, Bakamjian L. Women's growing desire to limit births in sub-Saharan Africa: meeting the challenge. Glob Health Sci Pract. 2013;1(1):97-107. http://dx.doi.org/10.9745/GHSP-D-12-00036

21) Paauw ND, van der Graaf AM, Bozoglan R, et al. Kidney function after a hypertensive disorder of pregnancy: a longitudinal study. Am J Kidney Dis. 2018;71(5):619-626.

22) Vikse BE, Irgens LM, Leivestad T, Skjaerven R, Iversen BM. Preeclampsia and the risk of endstage renal disease. N Engl J Med. 2008;359(8):800-809.

23) Stillman IE, Karumanchi SA. The glomerular injury of preeclampsia. J Am Soc Nephrol. 2007;18(8):2281-2284.

24) Craici IM, Wagner SJ, Bailey KR, et al. Podocyturia predates proteinuria and clinical features of preeclampsia: longitudinal prospective study. Hypertension. 2013;61(6):1289-1296.

25) Craici IM, Wagner SJ, Weissgerber TL, Grande JP, Garovic VD. Advances in the pathophysiology of preeclampsia and related podocyte injury. Kidney Int. 2014;86(2):275-285. 
26) Ayansina D, Black C, Hall SJ, et al. Long-term effects of gestational hypertension and preeclampsia on kidney function: Record linkage study. Pregnancy Hypertens. 2016;6(4):344-349. doi: 10.1016/j.preghy.2016.08.231 



\section{Part III}

Community-based approach to management of hypertensive disorders in pregnancy 


\title{
Chapter 7
}

Exploring survivor perceptions of pre-eclampsia and eclampsia in Nigeria through the health belief model

\author{
Pooja Sripad \\ Karen Kirk \\ Gloria Adoyi \\ Amy Dempsey \\ Salisu Ishaku \\ Charlotte E. Warren
}

Published in BMC Pregnancy and childbirth; (2019) 19:431. https://doi.org/10.1186/s12884-0192582-2 


\section{Abstract}

\section{Background}

In Nigeria, hypertensive disorders have become the leading cause of facility-based maternal mortality. Many factors influence pregnant women's health-seeking behaviors and perceptions around the importance of antenatal care. This qualitative study describes the care-seeking pathways of Nigerian women who sufferfrom pre- eclampsia and eclampsia. It identifies the influences-barriers and enablers - that affect their decision making, and proposes solutions articulated by women themselves to overcome the obstacles they face. Informing this study is the health belief model, a cognitive valueexpectancy theory that provides a framework for exploring perceptions and understanding women's narratives around pre-eclampsia and eclampsia-related care seeking.

\section{Methods}

This study adopted a qualitative design that enables fully capturing the narratives of women who experienced pre-eclampsia and eclampsia during their pregnancy. In-depth interviews were conducted with 42 women aged 17-48 years over five months in 2015 from Bauchi, Cross River, Ebonyi, Katsina, Kogi, Ondo and Sokoto states to ensure representation from each geo-political zone in Nigeria. These qualitative data were analyzed through coding and memo-writing, using NVivo 11 software.

\section{Results}

We found that many of the beliefs, attitudes, knowledge and behaviors of women are consistent across the country, with some variation between the north and south. In Nigeria, women's perceived susceptibility and threat of health complications during pregnancy and childbirth, including preeclampsia and eclampsia, influence care-seeking behaviors. Moderating influences include acquisition of knowledge of causes and signs of pre- eclampsia, the quality of patient-provider antenatal care interactions, and supportive discussions and care seeking- enabling decisions with families and communities. These cues to action mitigate perceived mobility, financial, mistrust, and contextual barriers to seeking timely care and promote the benefits of maternal and newborn survival and greater confidence in and access to the health system. 


\section{Conclusions}

The health belief model reveals intersectional effects of childbearing norms, socio-cultural beliefs and trust in the health system and elucidates opportunities to intervene and improve access to quality and respectful care throughout a woman's pregnancy and childbirth. Across Nigerian settings, it is critical to enhance context- adapted community awareness programs and interventions to promote birth preparedness and social support.

Keywords: Maternal health, Community awareness, Pre-eclampsia, Care-seeking behavior, Health belief model 


\section{Background}

Despite progress made in maternal health and shifting mortality patterns, evidence shows growing disparity around access to quality maternal care. It is essential to place perspectives, wants, and needs of individuals who seek and provide maternal health services at the center of shaping strategies to promote access to live-saving care [1].

\footnotetext{
"The true engine of change in maternal health...will be the determination of people at the front-lines of health systems patients, providers, and managers - to find or take the power to transform their own lived reality. Our job in global health is first to listen to them, and then to co-create the conditions at every level of the system that can make that locally drive transformation possible." [1]
}

In a world where 830 women die every day from pregnancy- or childbirth-related causes, persisting causes of death among women of reproductive age are hemorrhage (18\%), unsafe abortion (18\%), hypertensive disorders (12\%), sepsis (9\%), and other maternal disorders (22\%). Ninety-nine percent of these deaths occur in low- and middle-income countries and are more common among poor, rural women than urban women $[2,3]$. Preventing these deaths requires deeper investigation into the contributory causes of maternal mortality, the quality of care women receive, and their experiences during labor and delivery at health facilities.

In Nigeria, hemorrhage and pre-eclampsia/eclampsia make up over $50 \%$ of the country's maternal mortality. With strides in preventing hemorrhage-related deaths, hypertensive disorders have become the country's leading cause of maternal mortality, accounting for $29 \%$ of these deaths in tertiary hospitals [4]. A study conducted in Ogun State, Southwestern Nigeria found that location, time, obstetric condition, and socio-cultural characteristics influence health-seeking behaviors among pregnant women [5]. This study also showed that while some women preferred accessing health services at facilities, most preferred traditional doctors, healers, religious care, and traditional birth attendants during pregnancy and delivery. A lack of financial resources further delays seeking care, as women depend on their husbands to provide money for health services. Family members play a significant role in women's ability to access health care during pregnancy [6]. Since families consider pregnancy to be a natural part of life, they underestimate the importance of antenatal care (ANC) and the severity of danger signs and symptoms of pregnancy-related complications. Despite their social proximity to a care seeking woman, limited qualitative research exists on the influence of family 
members -spouses, parents, guardians and others - and community members on maternal careseeking decisions in Nigeria.

\section{Health belief model}

The health belief model is a cognitive value-expectancy theory that provides a framework for understanding women's perceptions of pre-eclampsia-related care seeking. In linking sociodemographics to perceptions and cues to action, the health belief model asserts that beliefs and attitudes predict health-seeking behaviors. The health belief model enables us to understand perceived susceptibility and perceived severity or threat of a specific health problem as well as the perceived benefits of and perceived barriers to its recommended solution [7]. It also considers 'cues to action' and the notion of self-efficacy in decision-making around a specific behavior. In many cultures, Nigeria included, pregnancy is a shared experience by a woman and her family. Studies that apply the health belief model to understanding women's actions during pregnancy explore healthy eating and being physically active as behaviors of focus and suggest that women's knowledge of what to do during pregnancy influences their perception of severity/ threats of health risks $[8,9]$. In the scenario of maternal health, pregnant women are more likely to engage in a specified behavior when perceived benefits override barriers. To our knowledge, the health belief model has not been used to look at pre-eclampsia and eclampsia nor to under- stand socio-cultural influences in Nigeria. This study describes the care-seeking pathways of Nigerian women who suffer from pre-eclampsia and eclampsia. It identifies the influences - barriers and enablers - that affect their decision making, and proposes solutions articulated by women to overcome the obstacles they face.

\section{Methods}

This study adopted a qualitative design that fully captures the narratives of women who experienced pre-eclampsia and eclampsia during their pregnancy. Data were collected over a five-month period from April to August 2015 in seven states across Nigeria: Bauchi, Cross River, Ebonyi, Katsina, Kogi, Ondo and Sokoto. Selected states include representation from each geo-political zone, covering the cultural diversity, varied socioeconomic development and differential access to health care services. Forty-four individual in-depth interviews (IDIs) were conducted with women who experienced preeclampsia, purposively selected and recruited in the community through health facility referrals by community health ex- tension workers. Participants were identified as survivors of pre-eclampsia by health care providers who managed - provided some level of care and referral - during women's labor 
and deliveries. While survivors likely experienced danger signs, they all received care either during ANC, childbirth, and/or in the early postpartum period. Eligibility criteria was not restricted to women who delivered in facilities nor differentiated on progression to eclampsia prior to care-seeking. The data collection team used a contact-tracing approach and engaged local guides to recruit women and interview them in the community. Data collectors experienced in qualitative methods and reproductive health were trained on study topics, interview guides, and research ethics. Data collectors had no prior established relationship with study participants. Instruments were pre- tested during the training. Women were asked open-ended questions related to their pregnancy, delivery, and post-natal experiences using a structured guide (Additional file 1) that included probes on quality of ANC, knowledge of pre- eclampsia and eclampsia, and factors that affect care seeking at individual, household, community and health systems levels. Socio-demographic information was collected to contextualize our findings. After obtaining written informed consent from participants, data collectors conducted interviews in local languages including Hausa (Bauchi, Katsina, Kogi, and Sokoto), Yoruba (Ondo), Igbo (Ebonyi), and Ibibio (Cross River). Care was taken to interview women in private settings. Two people attended each interview; one conducted the interview and the other took field notes.

Interviews were audio-recorded, transcribed verbatim and translated into English. With a grounded theory orientation, after an initial reading of the transcripts, a code structure was inductively developed, discussed, and applied to the data using the NVivo 11 qualitative software by two researchers. Memos written while coding the data allowed researchers to describe similarities and differences in women's perspectives between states, by age of marriage and parity, as well as emergently relevant characteristics. Through a deliberative process, researchers further grouped codes into themes and analyzed the local findings in dialogue with the health belief model.

Ethical approval for this study was granted by the Population Council's institutional review board (Protocol \#693), the National Health Research Ethics Committee of Nigeria, and research ethics committees from each study state.

\section{Results}

The 42 women interviewed were aged 17-48 years (median age: 30 years), married on average around the age of 21 years (range 13-37 years), and had between 0 and 8 children (Table 1 ). Women who married under 18 years of age (or 'early') lived in the northern states (Bauchi, Katsina and 
Sokoto) where the median age at marriage among our sample was 17 years (range: $13-25$ years). Of the 27 women who provided information on their level of education, 7 did not attend any school, 3 completed primary, 8 completed secondary, and 9 completed a higher degree (associates, bachelors, masters). The majority (39/42) of respondents attended ANC for at least one of their pregnancies. Those who did not attend ANC were teenage (aged 17-19 years) or older (36 years) mothers. The 32 respondents whose narratives approximated timing of first ANC visit during their recent pregnancy sought services at various stages $(n=12, n=15, n=5$ in the first, second and third trimesters, respectively). Women who only received ANC in their third trimester were young (4/5 were 17-20 years). Out of the 42 women who experienced pre-eclampsia, 18 developed eclampsia and 8 experienced perinatal death. Out of the 18 women who developed eclampsia - often reporting loss of consciousness as proxy for a seizure, 8 recalled it occurring at home, 2 mentioned during transit or in a village setting, 4 described it taking place at a hospital, and 6 did not specify. 
Table 1. Characteristics of Women WhoExperiencedPE/E

\begin{tabular}{|c|c|c|c|}
\hline & $\begin{array}{l}\text { Northe } \\
\mathrm{rn}\end{array}$ & $\begin{array}{l}\text { Souther } \\
n\end{array}$ & $\begin{array}{l}\text { Tota } \\
1\end{array}$ \\
\hline \multicolumn{4}{|l|}{ Age at interview } \\
\hline$\leq 17$ & 5 & 0 & 5 \\
\hline $18-29$ & 11 & 2 & 13 \\
\hline $30-48$ & 6 & 16 & 22 \\
\hline Missing & 1 & 1 & 2 \\
\hline \multicolumn{4}{|l|}{ Age at marriage } \\
\hline$\leq 17$ & 10 & 1 & 11 \\
\hline $18-29$ & 9 & 10 & 19 \\
\hline $30-37$ & 1 & 5 & 6 \\
\hline Missing & 3 & 3 & 6 \\
\hline \multicolumn{4}{|l|}{ Education } \\
\hline None & 7 & 0 & 7 \\
\hline Primary & 3 & 0 & 3 \\
\hline Secondary & 4 & 4 & 8 \\
\hline Higher Degree & 2 & 7 & 9 \\
\hline Missing & 7 & 8 & 15 \\
\hline \multicolumn{4}{|c|}{ Number of Children } \\
\hline 0 & 4 & 1 & 5 \\
\hline $1-2$ & 10 & 8 & 18 \\
\hline $3-4$ & 5 & 6 & 11 \\
\hline $5+$ & 3 & 2 & 5 \\
\hline Missing & 1 & 2 & 3 \\
\hline \multicolumn{4}{|l|}{ Age of youngest } \\
\hline$<1$ year & 9 & 5 & 14 \\
\hline $1-5$ years & 4 & 3 & 7 \\
\hline $5+$ years & 0 & 4 & 4 \\
\hline Missing & 10 & 7 & 17 \\
\hline \multicolumn{4}{|l|}{ Birth Outcome } \\
\hline Alive & $17^{\wedge}$ & 14 & 31 \\
\hline Perinatal Death & $4^{\wedge}$ & 4 & 8 \\
\hline Missing & 4 & 1 & 5 \\
\hline
\end{tabular}

"Two cases of twin births where one baby survived, and one died

We found that many beliefs, attitudes, knowledge and behaviors of women are relatively consistent across the country, with some variation among those in the north who married and became pregnant early. Nigerian women's perceptions of the susceptibility and threat of health complications during pregnancy and childbirth, including pre- eclampsia and eclampsia, were moderated by a number of factors including individual characteristics, medical histories and knowledge of causes, cues to action (awareness to seek care, trust in the health system, and family and community support), as well as perceived barriers (mistrust of health systems, socio-cultural 
barriers) and benefits (maternal/ newborn survival) to seeking timely care influence care- seeking behaviors (Fig. 1).

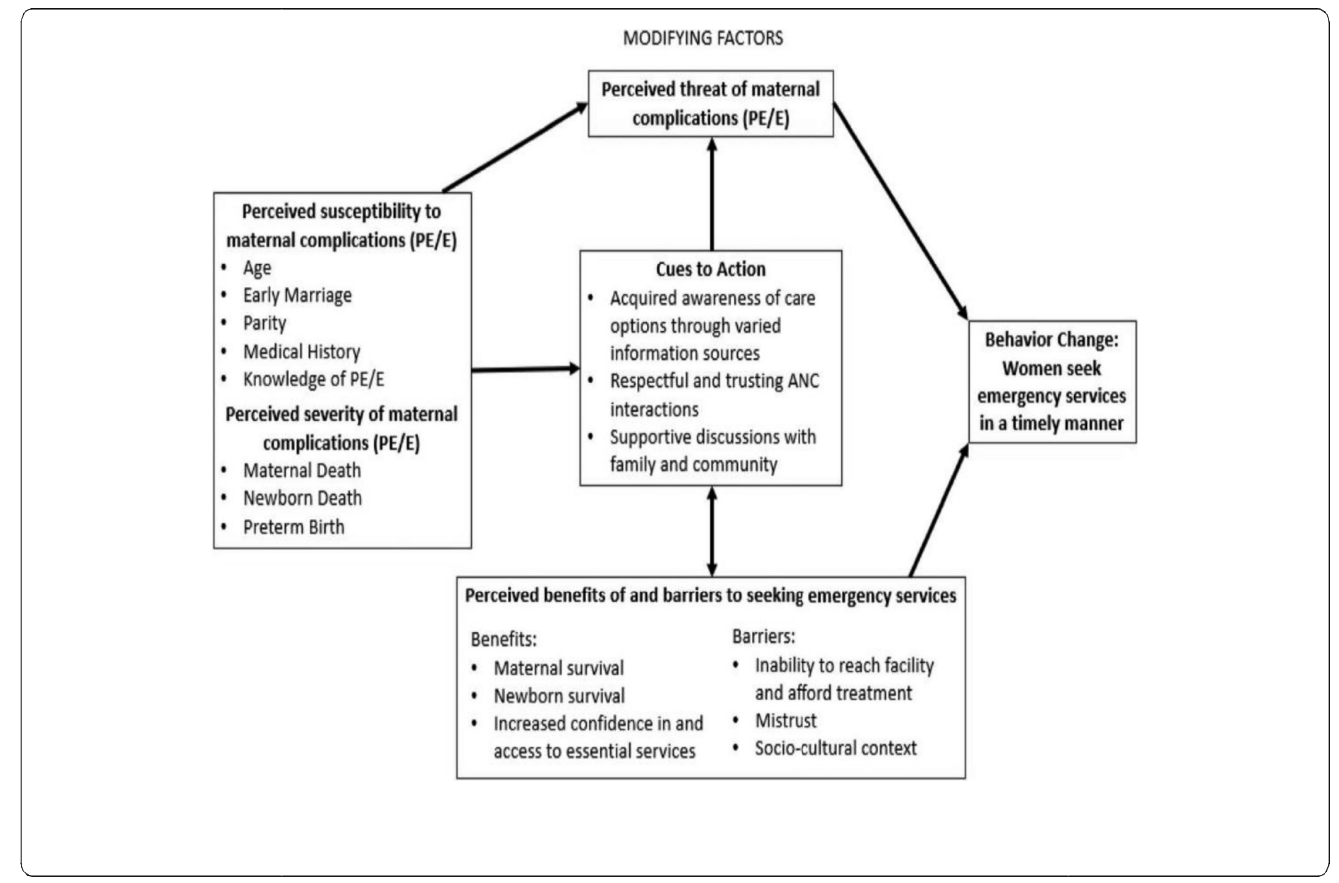

Fig. 1 Nigerian women's perspectives on care-seeking for pre-eclampsia and eclampsia applying a modified health belief model. *Adapted from (Champion \& Skinner, 2008) 


\section{Perceived susceptibility, severity and threat}

Women's perceived susceptibility of maternal health complications (e.g. pre-eclampsia and eclampsia) reflects their sense of risk - namely, their internal calculation of how likely they are to suffer complications. Perceived severity of these complications includes maternal and newborn death, as well as preterm birth; the combined notions of susceptibility and severity capture women's perceived threat of pre-eclampsia and eclampsia and its consequences. Though women's narratives suggest perceived susceptibility are often influenced by age, early marriage and/or parity, knowledge, and personal or family medical histories, a prevailing lack of comprehensive knowledge on pre-eclampsia and eclampsia (including danger signs and symptoms, causes, management, counseling, health risks during and after pregnancy) emerged across all states.

\section{Individual characteristics, histories and knowledge of causes and implications}

Variable knowledge of the causes of hypertension in pregnancy persist. Most women describe pre-eclampsia and eclampsia as brought on suddenly by stress (stress caused by household problems, normal activity, or pregnancy) or by 'thinking too much'; however, quite a few women report not knowing about these complications and related implications for maternal and newborn health outcomes.

\footnotetext{
"The first time I registered for antenatal I was told my BP [blood pressure] was too high so I never thought it was a problem, I thought it was a normal stress or maybe it was due to stress during pregnancy...nobody told me that it was a problem." (Ondo, 2 children)

Early marriage, common in the north (e.g. Katsina), appears associated with less comprehensive knowledge of causes and implications of pre-eclampsia and eclampsia potentially tied to women's limited educational status. In contrast, in the south (e.g. Cross River), women describe the notion of spiritual interference and witch- craft as a cause of preeclampsia and eclampsia.
} 
"When I first took - in [conceived] this time around, there was no problem just like in the case of other normal pregnancies. I thought it will equally go well, but to my surprise, things started changing as the pregnancy got older. I started with fever, later headache and dizziness. I did not go to hospital or ANC yet." (Katsina, 5 children).

"Sometimes they will tell you it's spiritual. They will tell you, a pregnant woman is supposed to be like this, be like that. Seek spiritual attention from traditional whatever..." (Cross River, 4 children).

In all states, one's own medical and family history as well as exposure to ANC messaging significantly modified perceptions of susceptibility and threat of incurring pre-eclampsia and eclampsia as a complication and risk of maternal death. Women who previously experiencedor had a family member who experienced - pre- eclampsia and eclampsia during childbirth were primed in perceiving the severity of complications that manifest as a real threat compared to first time pregnant women.

"My mom died of high blood related illness, [...] I remember when I was a kid, my mother during her sixth pregnancy was operated upon, and she lost the baby and was unconscious for some time and after that she couldn't take in [conceive] again. I don't know whether it was [high BP] or pre-eclampsia. Eventually she died of hypertension. My immediate younger sister had the same problem before she took in [conceived] and during pregnancy though she delivered safely through cesarean section." (Cross River, 2 children)

As the 40-year old woman with two children recounts, her perceived susceptibility to preeclampsia and the severity of negative consequences drew primarily from her family medical history. This woman went on to attend ANC and access emergency obstetric care where she delivered by cesarean section. She also reflected on her own personal history of hypertension in pregnancy.

"In the third pregnancy, the same thing occurred. My BP went up again in the first trimester and I went to the
hospital early and was placed on drugs, nifedipine and aldomet and it went down a bit, but later went up and I lost
the pregnancy. The experience of losing the pregnancy even triggered it the more...the fourth time I registered
early because of the high BP problem." (Cross River, 2 children)
Most women who attended ANC for one child continued to do so for subsequent pregnancies
and had greater knowledge of pre-eclampsia and eclampsia, suggesting that ANC plays a critical
role in birth preparedness, threat comprehension, and care seeking for obstetric emergencies. 


\section{Cues to action}

Cues to action on the perceived threat are broadly situated in enabling events or discussions that influence care seeking for pregnancy complications, including pre-eclampsia and eclampsia. Acquired awareness of care options Information sources for women on maternal complications and comprehension of danger signs and symptoms of pre- eclampsia and eclampsia - including the engagement of PHC providers, such as community health extension workers that helped with participant recruitment and provided ANC-emerge as cues to action. Comparing narratives from the south and north, we found that irrespective of education status, age of marriage, parity, and ANC attendance, women maintained similarly limited awareness of the signs and symptoms of pre-eclampsia and eclampsia, a perceived barrier to care seeking. This widespread lack of awareness points to concerns around quality and content of ANC messaging on clinical manifestations of maternal complications and overall birth preparedness. Despite generally knowing that high blood pressure is a potential problem during pregnancy, few women fully understood the significance of their own hypertension and the subsequent risk to their labor and delivery. Similarly, they did not comprehend the possible severity (i.e. maternal/newborn death). The following examples show how awareness of pre-eclampsia and eclampsia affects the quality of ANC interaction and learning; the first woman describes her inability to ask the right questions to health workers during ANC and the second describes the need for awareness- building, through media, community-focused intervention, and PHC provider-provided ANC as a cue to action.

"I did not attempt asking it [effect of high blood pressure] because I didn't know things of this nature [implications of BP] can come up." (Kogi, 4 children)

\footnotetext{
"Awareness is very important; if awareness is created through the media, and when we are talking about preeclampsia/eclampsia, it should be in simple language that the common person understands [...] When they go for ANC, women should ask the care givers of drug to take to prevent high BP especially those that have a history of high BP in the family... If pregnant women are enlightened that when they go for ANC, they should ask the nurses, 'since my mother had these issues, is there anything you can give me so that I will not develop the same problem?'” (Cross River, 2 children)
} 
Mixed quality of care at ANC in each state affects the extent to which women with preeclampsia/ eclampsia know where and when to seek emergency obstetric services. Though most women said that their blood pressure was taken, or urinalysis conducted, providers rarely explained results and implications. Often women describe having been prescribed blood pressure or other routine medications (e.g. antihypertensive, paracetamol, malaria) without adequate explanation. Consequently, not all women attributed their pregnancy risk to high blood pressure. No participants describe having abnormal urine - urinalysis results in relation to pre- eclampsia/eclampsia was never followed up during provider-patient discussions.

\section{Respectful and trusting ANC interactions}

Women's descriptions of their ANC and delivery experiences related to the technical quality of health services and respectful and trusting maternity care interactions, a cue to care-seeking action. Some women spoke about available (free) essential drugs for high blood pressure and supplies for safe delivery in government facilities precursors to quality of care and trust. However, as alluded to in the prior sub-section, when women did not receive ad- equate explanation about their condition or about any prescribed medications including potential side effects, their trust waivers, and cues to action (management of condition and careseeking in the future) are jeopardized.

"In the hospital, they advise and prescribe drugs for patients. But some patients tend to stop taking the drugs because of some side effects experienced... some don't buy the drugs. Usually, hypertensive drugs cause different things, if you don't tell what the drug reactions are, they wouldn't know. So, some decide to stop the drugs without coming back to hospital to explain what they feel when the drugs are swallowed." (Sokoto, no child survived)

Respectful maternity care affects care seeking for ANC and delivery as women fairly consistently describe positive experiences influencing their behavior.

\footnotetext{
"Then, the nurse that took my labor I would say she was an angel, God sent. She was just too nice, and she delivered my baby safely. I went home and I never thought it was a problem." (Ondo, 2 children, 41 years)
} 


\begin{abstract}
"They attend to me, my first day, they attended to me very well. The second attempt they [also] attended to me very well. I went there, and my BP was high. I needed and asked where I can lay my head and gather myself. They later attended to me." (Ebonyi, 7 children)

Women's narratives suggest that their trust in the health system's ability to manage maternal complications shifts over time and is determined by internalizing one's own experience during ANC, delivery and postnatal care as well by learning from others' experiences. Socio-cognitive processing of a woman's positive interaction with health providers and systems over the course of her pregnancy, including, receiving appropriate, timely, and respectful, non-judgmental services enhances trust and motivates care seeking. When women have or hear about negative experiences at health facilities, their trust in the system breaks down and delays their decision to seek emergency obstetric care. The following example describes a scenario in which a woman's perception rooted in community norms and perceptions shifted with her own care experience.
\end{abstract}

"Since I have been coming [to ANC], this caring helped save the baby and prevent me from this suffering. We were thinking everything is too costly and the staff might abuse us, but this is not what I found here." (Sokoto, 1 child)

This woman's shift toward respectful and trusting patient- provider relationships suggests that despite her and her husbands' perceived barriers to care - unfriendly providers and high cost - positive and caring interactions play a role in changing attitudes.

\title{
Supportive discussions with family and community
}

Engaging in discussions with family and communities emerged as a critical cue to action as these groups often provide instrumental support in women's ANC and delivery care seeking. Most women describe interactive discussion-based decisions with their spouse during pregnancy (before childbirth), suggesting husbands have significant influence on whether a woman gets timely care. Mothers-in-law and others in the household were also influential - in the cases of delivery, most women delayed care seeking till they passed out and a family member (spouse, uncle, mother, father, or sibling) brought them to the facility for care. Some women spoke of health providers and community members as influential, but less so than immediate family. Two women in northern states of Bauchi and Katsina decided on their own to seek care. 
"My husband used to tell me to relax and that with time it would go. My mother-in law sometimes encouraged me and sometimes she would talk as if I'm trying to be lazy but good friends would encourage me to always visit my doctors for check-up and other treatment." (Ebonyi, 1 child, 31 years)

"They prescribed some drugs; we bought them even though they were bit expensive. Some my husband bought outside the hospital." (Sokoto, no children/ currently pregnant)

Some believed that communities and churches ought not to bear influence since maternal complications relate to the private sphere of family decision-making, while others felt they should play a role in motivating timely care seeking and promoting ANC in addition to offering healing prayers. Perceived barriers and benefits to seeking timely care Perceived barriers to seeking timely care include an inability to reach facilities and afford treatment, mistrust of the health system, and socio-cultural context that affect a women's decision-making to seek emergency obstetric care, considering the perceived threat of complications.

\section{Inability to reach a facility and afford treatment}

Though not unique to these women or this context, in- adequate transportation options and significant costs emerged as significant transportation and financial barriers. In contrast to some women who described living near facilities, many lamented that long distances, challenging terrain, and inability to access vehicles prevent women from seeking services.

"The distance from the house to where the hospital is, is far, then I was ready to go to that hospital because of the distance, I was not able to make it, I delivered in the house." (Kogi, 4 children)

"[...] later the severe headache I was having surfaced again. I was unconscious, and my husband was out of town, there was no vehicle to convey me to the hospital. I was lucky to be conveyed by a government driver who was coming back from the farm." (Bauchi, 8 children)

Financial barriers affect access to transportation and affordability of health services or medicines. Some women in Bauchi, Sokoto, Kogi, Katsina, and Ebonyi experienced financial difficulty in securing transportation to the health facility while others felt that the prescribed drugs or necessary services were expensive. Women in Cross River did not experience the same financial burden for health services, noting that the drugs were affordable, and treatment was provided free of cost. 
"One day when the pregnancy was about 9 months, fever gripped me with severe headache, my body started shaking, I did not go out, I send for my husband, he came and said he will look for money to take me to the hospital, after some time, back pains started and I don't really know what was happened again." (Katsina, 5 children)

"We have no vehicles around and no money to travel to the Hospital. My husband suggested I went on my foot to the maternity near our village, I did." (Bauchi, 4 children)

\section{Mistrust}

Mistrust of the health system is often rooted in disrespect and abuse during childbirth either experienced or heard about in a community. Harsh or dismissive attitudes of providers toward women (self or others) in the maternity ward, coupled with negative childbirth out- comes - as often occurs with women who experience pre-eclampsia and eclampsia - may perpetuate mistrust of providers and the health system more broadly.

" $80 \%$ of nurses are hostile. They talk anyhow, they don't count you as somebody. During my delivery, I went through hell..." (Ondo, 2 children)

"The doctor or the nurse would be quarrelling that they don't want to see her [laboring woman]. [...] The doctor [...] said that the woman is not yet ready to deliver, that she should go and walk around... the woman said that this baby is coming down and they force the woman to go out of the labor ward to go and climb steps. In the process of climbing that step, the baby fell out and broke his head [...] before the woman could know, the baby is already dead. [...] the mother was crying the other nurse came and tell her to pay them their bill. Bill for what? For the dead child?" (Ebonyi, 2 children)

\section{Socio-cultural context}

While spirituality and tradition affect women's lives profoundly, they rarely factored into our sample's actual care- seeking decisions. Women often (particularly in the south) describe the importance of offering prayers (by a third party) to promote well-being of a woman experiencing pre- eclampsia.

"...despite that God is helping us we depend on that doctor..." (Ebonyi, 2 children)

One woman described going to a traditional birth attendant and few mentioned using traditionalist/herbalists, but the majority did not use or even mention them. 
"I never attended antenatal care throughout my pregnancies including this recent one. In this current pregnancy, I was well from the beginning until when it was six months when I began to have severe headache few days later, I noticed that my legs had swollen up. They gave me some herbs, the headache goes and comes back, it did not stop completely." (Sokoto, 3 children)

"The church believes everything is a spiritual attack and as a result, they keep you there until you pass out." (Cross River, 2 children)

As the above quotes suggest, confusion around spiritual and biomedical causes of complications alongside norms of using traditional remedies may delay necessary care seeking of women experiencing danger signs and symptoms. These complex socio-cultural dynamics manifest through interactions of church and community with families and spouses as primary decision-makers.

Perceived benefits influence care-seeking behaviors

The perceived benefit of a positive childbirth outcome (maternal and newborn survival) reflects the end goal for survivors of preeclampsia and eclampsia and requires balancing barriers with motivating cues to action, that enable women's care seeking. Our sample of women who experienced pre-eclampsia consistently describe the outcome benefits of accessing skilled care, though some articulate the merits to continuity of care as a part of developing trusted relationships that would enable better recognition and management of complications.

"My mind was telling me that if I went to that hospital [where] I delivered before, I won't have had such problem [...] I'm advising other women [...] please register in a hospital early as they notice they are pregnant and [...] stick to that particular place so that the doctors who started the antenatal would know the situation of their delivery and if there is a problem. They should tackle it at early stage [...] Both mother and child would come out alive and healthy." (Ebonyi, 2 children)

Survivors also describe candidly an elevated confidence in the health system's ability to care for them in an emergency as perceived benefit of care seeking - particularly as women receive ANC earlier and are empowered by programming that increases their knowledge of danger signs and symptoms of pre-eclampsia and eclampsia. Survivors also suggest a forward-looking 
community benefit of increased access to essential emergency services, if community-level education and mobilization.

"At the community level, I think we need to do more awareness programs for patients so that if they have hypertension, they don't play with it. It's not only eclampsia that it leads to." (Kogi, 4 children)

\section{Discussion}

Dialoguing Nigerian women's narratives through a modified health belief model reveals how perceived susceptibility to pregnancy complications relates to perceptions of severity or threat of pre-eclampsia and eclampsia and subsequent care-seeking behavior. Our data also show how this pathway is influenced by a range of modifying factors, including cues to action, and perceived barriers to and benefits of seeking emergency care. Findings indicate that enhancing women's and community knowledge of pre-eclampsia and eclampsia, danger signs in pregnancy, including supporting social responsiveness to symptoms and enabling access to care, advocating for early-stage interaction with health providers - particularly among younger primigravida women, and strengthening quality ANC interactions between women and providers may lead to positive care-seeking behavior and improved maternal and newborn health outcomes in Nigeria. These intervening points may increase trust and confidence in health systems. Relatively consistent findings across the north and south further suggest that these propositions can be leveraged as a guide and customized to specific gaps in a programming context.

Beyond the minor differences in perceived susceptibility and threat across respondents' age, other factors including age at marriage, parity, family medical history and knowledge levels (ANC and community-derived) strongly affected women's perception of pre-eclampsia and eclampsia. By linking prior pregnancies and others' experiences of pregnancy in which high blood pressure was a concern, respondents illustrate the social learning pathway that occurs around risk, consequences, and care options for pre-eclampsia and eclampsia. The low to moderate quality of ANC (insufficient explanation of clinical implications of blood pressure tests and urinalysis) and birth preparedness (what to do/where to go when danger signs experienced) described by almost all the women regardless of parity, age, or geographic region, 
triangulate this awareness gap around pre- eclampsia and eclampsia. Spirituality plays a larger role in the South, early marriage confounds associations in the North, and women in Cross River state (South) face fewer financial and transport barriers. (Mis)trust in health systems - including positive and negative inter- personal relationships with providers during ANC and childbirth that may extend into the postpartum period - and social support moderate women's experiences by affecting whether and how women weigh and act upon barriers and benefits to seeking emergency care.

\section{Gap in awareness}

Knowledge of causes and consequences of pre-eclampsia and eclampsia, in addition to awareness about when and where to seek care, affect a woman's perception of threat of developing pre-eclampsia as seen in our study and elsewhere. A study conducted in Sokoto, Nigeria interviewed 'relations' of women with eclampsia and found that only $7 \%$ of 159 interviewees correctly associated eclampsia with high blood pressure. Instead, they cited 'evil spirits', pregnancy, and God as causes and tended to seek traditional rather than biomedical treatment [10]. Another community- based survey collating binary responses from 200 adult women in Tanzania demonstrated the sample's significant lack of awareness (50\% or less across all knowledge items); only $13 \%$ knew epigastric pain to be a symptom of pre- eclampsia and half believed evil spirits and exposure to fire are contributory causes of pre-eclampsia [11]. A systematic review confirms that traditions, cultural beliefs, and social norms are common factors that affect perceptions of antenatal and obstetric care in sub-Saharan Africa [12]. It is critical to address this awareness gap and focus on how women are getting their maternal health information. Improving community awareness and understanding of pregnancy risks will allow them to perceive threat of pre- eclampsia and eclampsia and promote timely and appropriate care seeking.

\section{Inequitable financial burden}

Frequently, financial barriers affect access to transportation, availability of drugs, and affordability of services. Many women did describe costs to affect their ability to access care. Respondents from Cross River consistently received free services or paid nominal amounts for 
their care, possibly related to the public-private partnership, Saving Mothers Giving Life, that works to strengthen the health system and improve maternal and newborn health outcomes [13]. Though Nigeria has made efforts to set up a National Health Insurance Scheme, there has been inconsistent success in reducing out-of-pocket expenditure.

\section{Trust and experience}

The modifying influence of trust and positive experience in contrast to mistrust and negative experience described by Nigerian women is critical to improving the continuum of care received throughout pregnancy (ANC and labor and delivery), particularly in the timely management of complications like pre-eclampsia and eclampsia. The way in which trust and mistrust in the health system motivates and reinforces care-seeking are growing areas of investigative interest in sub-Saharan Africa and in maternity care specifically [14, 15]. Globally, the recognition that trust or confidence in a health system is part of perceived health care quality, further emphasizes the need to understand the mechanisms and measurement of these concepts in practice [16]. The experience of hostile and dismissive attitudes shown by health providers in our study echoes forms of disrespect and abuse described elsewhere in Nigeria [17]. While quality of ANC in Nigeria remains limited and non-comprehensive [18], more research is needed around how trust (and mistrust) interact with experience and outcomes of antenatal, delivery, and postnatal care in Nigeria.

\section{Limitations}

The analysis only considered the perceptions of women who experienced pre-eclampsia. The study team was therefore unable to draw comparisons between those who did and did not experience pre-eclampsia regarding their perceived susceptibility, perceived threat, cues to action, perceived benefits and timely care seeking. However, as maternal recall among women who suffer complications may be similar or higher compared to a general population, the findings maintain credibility. Another limitation was insufficient probing into select themes of spirituality and prayer and disrespect and abuse. Articulation of nuanced mechanisms through which these concepts affected care-seeking would have allowed for discerning religious reasons or specific traditional practices leading to delays in care-seeking decisions and behavior 
among women who experienced pre-eclampsia/eclampsia (and/or their families). All the narratives could have probed further into the context, type, frequency, and determinant of the disrespectful, abusive, or respectful experiences during ANC and labor and delivery.

The health belief model helps explicate what factors motivate individuals to engage in a specific action, it limits commentary to that one action, as opposed to distinguishing future intentions and behaviors. Influence of social norms and the gap between intent and actual behavior change cannot be fully captured in health belief model but arise as an issue in Nigeria. Future research should consider the Theory of Normative Social behavior [19] or similar models that consider the influence of descriptive and injunctive norms and interpersonal communication on individual's health-seeking behavior.

\section{Implications}

Despite these limitations, study findings reinforce a need to address educational deficiencies, health care attitudes, and access to resources at the community level to effectively harness cues to action and mitigate the barriers faced by women to seek care. Connecting women with timely care in this context requires:

- Increasing awareness of hypertension in pregnancy through community-based health promotional activities that leverage locally relevant and trusted forums

- Improving quality of ANC at primary health care level, closer to where women live; and

- Strengthening primary health care givers' ability to manage hypertension in pregnancy and make timely referrals to hospitals.

Community-focused interventions that emphasize birth preparedness for all women during their pregnancy and adopt social behavior change approaches render cues to action (in case of a complication) more interpretable [20]. In Nigeria, the role of communities and social leaders in dispelling myths, translating knowledge of hypertension in pregnancy, and link women to quality care at primary health care facilities ought to be leveraged. Group ANC and women's groups are potential platforms to improve health literacy, care-seeking attitudes, and use of necessary antenatal and delivery services $[21,22]$. Affording access to financial resources that 
enable women to reach facilities at the community level in Nigeria requires evaluation of the functionality of National Health Insurance Scheme, though could also draw from regional work demonstrating increase in maternal service use among women participating in micro-finance groups $[23,24]$. Public policies enhancing primary health care givers' capacity to manage preeclampsia and linking women to a functional referral system that does not rely solely on a family's financial ability is critical to facilitating care use.

\section{Conclusion}

This study reveals that social-behavioral theory offers useful perspective to understanding careseeking pathways in maternal health, illuminating the effects of childbearing norms, sociocultural beliefs and trust in the health system on women's decisions in northern and southern Nigeria. Where women's understanding of their susceptibility to and threat of pregnancy complications including pre- eclampsia is limited, the health belief model elucidates opportunities to intervene and improve access to antenatal and emergency obstetric care. Across Nigerian settings, the cues to action identified in our study - as they relate to the benefit of positive health outcomes and greater access to essential emergency services - offer pragmatic implications for investment in (a) programs that enhance context-adapted community awareness-building approaches to promote birth preparedness and social support and (b) development and implementation of national policies emphasizing respectful quality care throughout pregnancy and childbirth.

\section{Abbreviations}

ANC: antenatal care; BP: blood pressure; HBM: Health Belief Model

Acknowledgements

We thank USAID for their feedback and funding the Ending Eclampsia Project's landscape analysis that provided the empirical data analyzed in this manuscript.

Authors' contributions

PS conceived of this manuscript, its design and analysis, drafted and revised the paper. KK supported conceptualization of the manuscript, participated in analysis, and contributed to writing and revising the manuscript. CW and SI participated in conceptual design and coordination of the overall Ending Eclampsia study 
and reviewed the manuscript. GA coordinated data collection and supported codebook development and analyses along with AD. All authors read and approved the final manuscript.

Funding

Data analyzed in this manuscript were collected under the Ending Eclampsia project funded by the United States Agency for International Development (USAID) under the terms of the cooperative agreement USAID AID-OAA14- 000048. USAID funded researchers' time and data collection activities and were involved in a final review of the manuscript. USAID was not directly involved in the study design, data collection, analysis and interpretation, nor writing of the manuscript.

Ethics approval and consent to participate

All study participants provided written informed consent. Ethical approval for this research protocol was granted by the Population Council's institutional review board (Protocol \#693), the National Health Research Ethics Committee of Nigeria, Cross River State Health Research Ethics Committee, Kogi State Health Research Ethics Committee, Katsina State Health Research Ethics Committee, Bauchi State Health Research Ethics Committee, Ondo State Health Research Committee, Sokoto State Health Research Ethics Committee, and Ebonyi State Health Research Ethics Committee.

Competing interests

The authors declare that they have no competing interests.

Author details

${ }^{1}$ Population Council, 4301 Connecticut Avenue NW Suite 280, Washington, DC 20008, USA. ${ }^{2}$ Population Council,

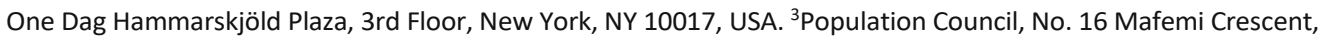
Utako District, Abuja, Nigeria.

${ }^{4}$ Julius Center for Health Science and Primary Care, University Medical Center, Utrecht University, Utrecht, Netherlands. 


\section{References}

1. Freedman LP. Implementation and aspiration gaps: whose view counts? Lancet. 2016;388(10056):2068-9.

2. Alkema L, et al. Global, regional, and national levels and trends in maternal mortality between 1990 and 2015, with scenario-based projections to 2030: a systematic analysis by the un maternal mortality estimation inter-agency group. Lancet. 2016;387(10017):462-74.

3. Graham W, et al. Diversity and divergence: the dynamic burden of poor maternal health. Lancet. 2016;388(10056):2164-75.

4. Oladapo $O$, et al. When getting there is not enough: a nationwide cross- sectional study of 998 maternal deaths and 1451 near-misses in public tertiary hospitals in a low-income country. BJOG. 2015.

5. Akeju DO, et al. Determinants of health care seeking behaviour during pregnancy in Ogun State, Nigeria. Reprod Health. 2016;13(1):32.

6. Fagbamigbe AF, Idemudia ES. Barriers to antenatal care use in Nigeria: evidences from non-users and implications for maternal health programming. BMC Pregnancy Childbirth. 2015;15(1):95.

7. Champion VL, Skinner CS. The health belief model. In: Glanz K, Rimer BK, Viswanath K, editors. Health behavior and health education: theory, Research, and Practice, 4th ed. San Francisco: Jossey-Bass; 2008. pp. 45-65.

8. Khoramabadi M, et al. Effects of education based on health belief model on dietary behaviors of Iranian pregnant women. Glob J Health Sci. 2016;8(2): 230-9.

9. Da Costa D, Ireland K. Perceived benefits and barriers to leisure-time physical activity during pregnancy in previously inactive and active women. Women Health. 2013;53(2):185-202.

10. Adamu A, Tunau K. The perception of patients' relations on the cause of Eclampsia. Gynecol Obstet. 2014;4(2):1-4.

11. Savage AR, Hoho L. Knowledge of pre-eclampsia in women living in Makole Ward, Dodoma, Tanzania. Afr Health Sci. 2016;16(2):412-9. 
12. Brighton A, D'Arcy R, Kirtley S, Kennedy S. Perceptions of prenatal and obstetric care in sub-Saharan Africa. Int J Gynecol Obstet. 2013;120(3):224-7.

13. Saving Mothers Giving Life, "2016 annual report, reducing maternal mortality in subSaharan Africa," 2016.

14. Østergaard L. Trust matters: a narrative literature review of the role of trust in health care systems in sub-Saharan Africa. Glob Public Heal. 2015;10(9):1046-56.

15. Sripad P, et al. Exploring meaning and types of Trust in Maternity Care in Peri-urban Kenya: a qualitative cross-perspective analysis. 2017.

16. Kruk ME, et al. The Lancet Global Health Commission High-quality health systems in the Sustainable Development Goals era: time for a revolution. no. 18, pp. 1-57, 2018.

17. Bohren MA, et al. Mistreatment of women during childbirth in Abuja, Nigeria: a qualitative study on perceptions and experiences of women and healthcare providers. Reprod Health. 2017:1-13.

18. Salomon A, Ishaku S, Kirk KR, Warren CE. Detecting and managing hypertensive disorders in pregnancy: a cross-sectional analysis of the quality of antenatal care in Nigeria. BMC Health Serv Res. 2019;19(1):411.

19. Rimal R, Lapinski M, Cook R, Real K. Moving toward a theory of normative influences: how perceived benefits and similarity moderate the impact of descriptive norms on behaviors. J Health Commun. 2005;10(5):433-50.

20. Brazier E, Fiorentino R, Barry S, Kasse Y, Millimono S. Rethinking how to promote maternity care-seeking: factors associated with institutional delivery in Guinea. Health Care Women Int. 2014;35(7-9):878-95.

21. Lori JR, Ofosu-Darkwah H, Boyd CJ, Banerjee T, Adanu RMK. Improving health literacy through group antenatal care: a prospective cohort study. BMC Pregnancy Childbirth. 2017;17(1):228.

22. Mangham-Jefferies L, Pitt C, Cousens S, Mills A, Schellenberg J. Cost- effectiveness of strategies to improve the utilization and provision of maternal and newborn health care in low-income and lower-middle-income countries: a systematic review. BMC Pregnancy Childbirth. 2014; 14:243. 
23. Abekah-Nkrumah G, Abor PA, Abor J, Adjasi CKD. Improving maternal healthcare utilisation in sub-Saharan Africa through micro-finance. Int J Health Care Qual Assur. 2011;24(8):601-10.

24. Lorenzetti LMJ, Leatherman S, Flax VL. Evaluating the effect of integrated microfinance and health interventions: an updated review of the evidence. Health Policy Plan. 2017;32(5):732-56. 


\section{Supplement I}

In-depth Interview Guide - woman who experienced pre-eclampsia/eclampsia

1. How old are you? How old were you when you got married (first time)? How old were you at the time of your first pregnancy?

2. Have you ever attended school/madrasa? What is the highest class you completed?

3. How many living children do you have? What is number/rank of the last child?

4. Are you currently pregnant? If yes, how old is your pregnancy?

5. What is the age of your last child (excluding the index child) When was your last child (child name) born? What was the mode of delivery? Where did you deliver your baby?

6. How far is the nearest government health facility and private hospital or clinic from your home?

7. Did you receive any ANC visit during current/last pregnancy? How many times did you receive ANC checkup in total? At what gestational age did you book this index pregnancy?

8. Where did you go for your first antenatal checkup? Who performed your first antenatal checkup? Did you go to a health facility mainly for antenatal checkup or because you have any other problem(s)? For what problem, if any, did you visit ANC?

9. When you went for ANC please can you tell me what happened during the consultation? Probe: did you have your BP measured or urine tested? Did the service providers inform you of the result of any measurement or test? If yes, did the providers explain to you significance of any finding? Can you remember what they told you your BP was?

10. Did the service provider inform you about the possible pregnancy related problems/complications? If yes, please tell me what they said.

11. Did the service provider refer you to another facility for your problem? If yes, for what complications were you referred? 
12. Did you receive any treatment for high blood pressure or seizures? If yes, where did you receive the treatment? Did your condition improved/seizure subside after receiving the treatment?

13. Who referred you to this facility? For what problems/complications did you come here? Could you explain what type of problems you were experiencing before coming to this hospital? Probe: Did you suffer from severe headache, blurred visions, high blood pressure, swelling feet, or seizures during your pregnancy time?

14. Were you aware that pregnant women may suffer from high blood pressure and or seizures? If yes, tell me what you know about this? Did any service providers tell you about the possibility? Probe: what about during ANC?

15. Now, could you briefly describe your experiences at these levels in your effort to seek care for this complication?

a) Family level, including interactions with your spouse and mother in-law?

b) Community members, including religious leaders and traditional service providers?

c) Health care facilities: how were you attended to? Availability of essential drugs, cost of the treatment and outcomes for you and your baby

a. What is your final advice on how you think the problem of high blood pressure and or convulsion in pregnancy can be dealt with at: a) Family level b) Community level c) Health care facility level? 


\section{Chapter 8}

\section{A primary health care model for managing pre-eclampsia and eclampsia in low- and middle- income countries}

Charlotte E. Warren

Sharif Mohammed Ismail Hossain

Salisu Ishaku

Deborah Armbruster

Emily Hillman

Published in Reproductive Health Journal; (2020) 17:46 https://doi.org/10.1186/s12978-020-0897-0 


\begin{abstract}
Background

Hypertensive disorders in pregnancy, specifically pre-eclampsia and eclampsia (PE/E), are the second biggest killer of pregnant women globally and remains the least understood and most challenging maternal morbidity to manage. Although great strides were made in reducing maternal and newborn mortality between 1990 and 2015, this was clearly not enough to achieve the global health goals. To reduce maternal deaths: 1) early detection of PE needs to be improved; 2) effective management of PE/E needs to occur at lower health system levels and should encourage timely care-seeking; and 3) prioritizing the scale up of a comprehensive package of services near to where women live.
\end{abstract}

\title{
Findings
}

This commentary describes a pragmatic approach to test scalable and sustainable strategies for expanding access to quality under-utilized maternal health commodities, interventions and services. We present a primary health care (PHC) $\mathrm{PE} / \mathrm{E}$ Model based on implementation research on identified gaps in care in several countries, accepted global best practice and built on the basic premise that PHC providers can take on additional skills with adequate capacity building, coaching and supervision, and community members desire control over their own health. The PHC $\mathrm{PE} / \mathrm{E}$ model displays the linkages and opportunities to prevent and treat $\mathrm{PE} / \mathrm{E}$ in a simplified way; however, there are numerous interlinking factors, angles, and critical points to consider including leadership, policies and protocols; relevant medicines and commodities, ongoing capacity building strategies at lower levels and understanding what women and their communities want for safe pregnancies. 


\section{Conclusion}

The PHC model described here uses PE/E as an entry to improve the quality of ANC and by extension the pregnancy continuum. Bringing preventive and treatment services nearer to where pregnant women live makes sense.

\section{Key message}

This model provides a pragmatic approach for improving access to antenatal services for detection, prevention and management of pre-eclampsia and eclampsia at the primary health care level. 


\section{Background}

In October 2018, in Astana, Kazakhstan, the global health community reaffirmed their commitment towards universal health coverage and made primary health care (PHC) a cornerstone of their attempt to reach the sustainable development goals (SDGs) by 2030 [1]. Al- though great strides were made in reducing maternal and newborn mortality between 1990 and 2015 (by 44 and 49\% respectively), this was not enough to achieve the SDGs [2, 3]. More than 830 women and 7700 newborns still die each day from pregnancy-related complications; an additional 7300 women experience a stillbirth with approximately $16 \%$ occurring in pregnancies complicated by hypertension [4]. Of the three direct causes of obstetric mortality (bleeding, sepsis and hypertensive disorders in pregnancy (HDPs) accounting for 27, 11 and $14 \%$ respectively) [5], HDPs, specifically pre- eclampsia and eclampsia (PE/E), remain the least understood and most challenging to manage [6]. Risks to mothers' cardiovascular and cerebrovascular health decades later and the inter-generational impact of HDPs are only now starting to be understood and remain unquantified in low- and middle-income countries $[6,7]$.

Building on the work and successful efforts to prevent and manage postpartum hemorrhage (PPH) $[8,9]$ over the last 10 years - donors and maternal health experts started to focus on other causes of maternal deaths. Globally pre-eclampsia (PE) is the second major killer of pregnant and postnatal women, but unlike $\mathrm{PPH}$, where approximately $70 \%$ can be prevented by administering oxytocin, PE is harder to recognize early and the treatment, magnesium sulphate (MgSO4), is only given to those with severe disease. To reduce maternal and perinatal deaths: 1) early detection of PE needs to be im- proved; 2 ) effective management of PE/E needs to occur at lower health system levels and should encourage timely care-seeking; and 3) prioritizing the scale up of a comprehensive package of services near to where women live [1014].

This commentary describes a pragmatic approach undertaken by a multi-country study (supported by USAID from 2014 to 2019 and implemented by Population Council, through the Ending Eclampsia project) to test scalable and sustainable strategies for expanding access to quality under-utilized maternal health commodities, interventions and services. The project 
brings an implementation science approach to catalyze focus on PE/E by building on evidencebased recommendations $[15,16]$. To understand the evolution of the development of the PHC model for PE/E presented here, we need to understand the prevailing practices prior to 2014.

Findings from the multi-country 'MAGPIE trial' in 2001 demonstrated that MgSO4 reduces the risk of eclamptic seizures by half among women with pre- eclampsia and reduces death from eclampsia [15]. Based on these findings, countries registered MgSO4 on their Essential Medicines List (EML) and introduced it to referral facilities. Although WHO recommends MgSO4 as the anticonvulsant for managing severe $\mathrm{PE} / \mathrm{E}$, (and methyldopa and hydralazine for controlling severe hypertension in pregnancy) $[16,17]$, the burden of calculating dosage of MgSO4 is daunting, time-consuming and can introduce errors, tempting providers to substitute diazepam for MgSO4, a less effective anticonvulsant [8]. Moreover, MgSO4 toxicity may result in respiratory failure and the need for the antidote, calcium gluconate. Even though less than $0.1 \%$ women die due to toxicity, there is a "fear" among providers making them reluctant to use MgSO4 [18]. More recently global advocacy efforts through the UN Commission on Life Saving Medicines for Women and Children 2012-14 focused on procuring quality MgSO4 and only one formulation of $\mathrm{MgSO} 4$ ( $50 \%$ or $5 \mathrm{~g}$ in $10 \mathrm{ml}$ ) to reduce the calculation challenges that exist [8].

Following the more widespread uptake of MgSO4 in referral facilities, a few studies also demonstrated the feasibility and acceptability of task-sharing management of PE to lower levels where providers administer a loading dose of MgSO4 correctly and refer the patient for further management $[6,19,20]$. However, WHO recommendations on task shifting or sharing maternal and newborn health (MNH) services do not include use of antihypertensives for severe hypertension for lower level cadres to prescribe [21]. Many countries are guided by WHO and therefore do not allow these cadres to prescribe and administer life-saving drugs for pregnant women with obstetric complications, yet this is where women often first seek care. Although 29/31 countries surveyed had MgSO4 on their EML in 2011, there is limited information available on specific antihypertensives for use during pregnancy in these countries [17]. 
Landscape analysis in five countries - baseline findings

In 2015-2016, we conducted landscape analyses in Bangladesh [10], Nigeria [11], and Pakistan [12] on access to PE/E services. Further in-depth assessments in Ethiopia and Kenya provided a broader picture of the provision and experience of care for PE/E across sub-Saharan Africa and south east Asia. Key findings included providers' poor knowledge and skills in addition to a lack of essential commodities and equipment. Facilities were more likely to have oxytocin for prevention and management of PPH than MgSO4 to manage PE/E. Specific gaps at the lower facility levels include lack of task shifting policy, weak referral policies, lack of access and use of antihypertensives; lack of postpartum monitoring; and lack of community awareness around $\mathrm{PE} / \mathrm{E}$ including danger signs. Headaches and blurred vision associated with $\mathrm{PE} / \mathrm{E}$ are perceived as malaria or 'overthinking' and swollen ankles are perceived as normal. Women who experienced $\mathrm{PE} / \mathrm{E}$ describe multiple contacts with the health system before receiving the necessary life- saving care [10-12]. The learning from the country level landscape analyses, in collaboration with input from key stakeholders - including in-country Ministry of Health officials, partners working in $\mathrm{MNH}$ and discussions with members of national and international technical working groups - formed the basis of a comprehensive PHC model for PE/E, tested through implementation research between 2016 and 2018. The model also built on evidencebased recommendations from WHO and other international experts [15, 20, 22].

The primary health care - pre-eclampsia/eclampsia model

We present a PHC PE/E Model (see Fig. 1) based on implementation research on identified gaps in care in several countries, accepted global best practice and built on the basic premise that PHC providers can take on additional skills with adequate capacity building, coaching and supervision, and community members desire control over their own health [1]. By using PE as a lens, we believe this model is transferrable across countries and practices along the pregnancy continuum with a particular focus on antenatal care (ANC). However, our research has also shown that without appropriate policies, sufficient finances, supplies/medicines in place and effective governance/ leadership, the success of the model may be limited [10-12]. Here, we 
describe the PHC model for PE/E care representing a set of critical interventions for early detection and quality PE/E prevention and management tested through IR:

Community level

- Use women's groups as a platform to increase awareness on danger signs and importance of early care seeking during pregnancy (Cross River state, Nigeria).

- Include country-specific community-based cadres: Community Midwives (CMWs) in Sindh Province, Pakistan, identified as capable providers of MgSO4 loading dose with referral.

\section{Primary health care}

- Use PE/E as a lens on quality of ANC:

- Encourage early care seeking behavior for enabling early detection of high blood pressure (BP).

- Raise awareness of signs and symptoms and what women should expect/ask at facilities during ANC and postnatal (PNC) contacts.

- Build capacity / improve performance of service providers:

- Mobilize master trainers who are available locally.

- Develop training plans, which include follow- up mentoring, coaching and supportive supervision, including refresher training as necessary during facility visits.

- Ensure competent and confident providers can identify, monitor, stabilize and refer patients to higher levels

- Ensure functionality and/or readiness of system:

Availability and use of equipment, and supplies for detection and treatment: BP machines, stethoscope, urine dipsticks, patella hammer, syringes, needles, etc. 
Ensure consistent supply of quality medicines for managing HDPs; antihypertensives (those readily available in country - labetalol, nifedipine, or alpha methyldopa), MgSO4, and calcium gluconate.

Engage pharmacy workers and logistics officers to ensure they understand the importance of consistent stock of equipment and commodities for obstetric complications.

- Provide quality care:

Inform women and families routinely on danger signs/symptoms of pregnancy. This could be done through individual visits or through group counseling.

- Check BP and urine albumin routinely to detect PE early [15, 22]

- If high BP or PE is identified, monitor closely, provide antihypertensives as appropriate

- If $B P$ is not controlled or severe PE/E ensues, stabilize with antihypertensive, loading dose of MgSO4 and refer to appropriate facility [16, 22, 23].

- $\quad$ Ensure hypertension is controlled:

- Use of a simple algorithm/clinical protocol: monitor BP closely.

- Prescribe anti-hypertensive for moderate to severe hypertension [24].

- Ensure referral system is in place:

- Assist or identify locally available transport. Referral notification with higher level facility. Ensure post-referral monitoring/follow-up 


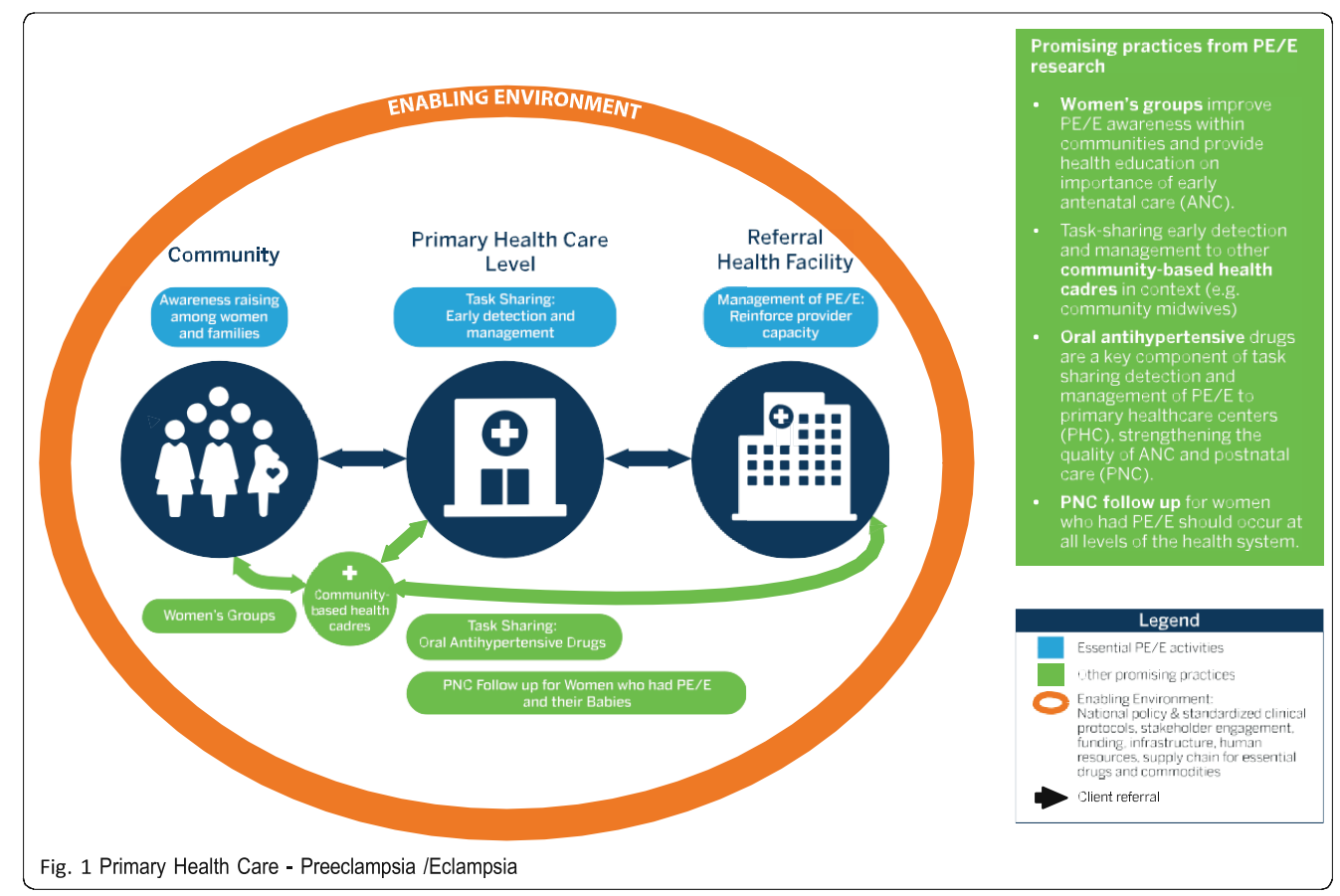

\section{Referral health facility}

- Functional referral facility (Emergency Obstetric and Neonatal Care):

- Ensure maternity units have received training on identification and management of $\mathrm{PE} / \mathrm{E}$

- Ensure use of nationally/internationally recognized treatment algorithms/protocols

- Ensure necessary equipment, and commodities, in place

- Ensure expertise and appropriate skills mix of staff available

Skilled providers to manage PE/E:

- Ensure providers are confident and capable to manage PE/E appropriately (as above)

- Assist local staff to create a functioning referral and counter referral plan

The model can be adapted to different country con- texts or tailored to country level. Here are the gaps to consider. 


\section{Critical issues to consider}

The Primary Health Care Pre-eclampsia/eclampsia (PHC PE/E) model (Fig. 1) displays the linkages and opportunities to prevent and treat PE/E in a simplified way; however, there are numerous interlinking factors, angles, and critical points to consider. The different elements of the model each play an important role and have different resource implications, when ensuring that women with $\mathrm{PE} / \mathrm{E}$ are treated quickly and appropriately. Policies must be in place that include task sharing/shifting to PHC providers to prescribe antihypertensive drugs for managing moderate to severe hypertension (systolic BP $\geq 160$ or diastolic $\geq \mathrm{BP}-110$ ) and administer the MgSO4 loading dose to women with severe PE/E. Standardized protocols need to be used all the time by everyone to ensure consistent management across the system [16, 22]. Leadership at facility, district, regional, national levels is critical to champion the implementation of the policy.

The medicines (MgSO4, calcium gluconate and antihypertensive drugs) recommended by WHO must be available where needed. Support to the public sector supply chain is required in order to have the right medicine, in the right place, at all times - and availability in the private sector or alternatives for when either system fails. Health care providers at PHC level must have the necessary equipment and logistics and the knowledge and skills to identify, provide initial treatment (loading dose of MgSO4 and antihypertensive) and refer [14, 24]. At referral facilities, providers should have the expertise and capacity for optimal emergency management of PE/E. Providers should benefit from refresher training, emergency drills/updates as needed, ongoing supportive supervision, and mentoring. However, it is important to recognize that task shifting or sharing to lower cadres only makes sense if those cadres already have basic clinical skills (for example taking BP, administering injections) $[22,23]$. Evidence shows that MgSO4 should not be 'pushed' to the lowest level in communities (i.e. community health workers) but stay within a clinical setting [25]. We cannot forget that the women and communities must be involved, they must be knowledgeable and able to advocate for themselves and to ensure providers are listening to them. It is important that they recognize the danger signs, attend ANC in the first trimester and have at least eight contacts to be screened for signs of PE in order to minimize the impact of this disease on themselves and their newborns. Community platforms 
- such as women's groups - can be leveraged to raise awareness about health topics, including $\mathrm{PE} / \mathrm{E}[26,27]$. Referral pathways must be well defined to achieve the best outcomes. During training, cadres (from consultants to lower level providers) at all levels require updating in the referral process to ensure women receive prompt care. Close monitoring of women during labor and delivery is critical as is monitoring during the immediate postpartum period when 20-30\% of hypertensive and eclamptic seizures occur and again before she leaves the facility, followed by comprehensive follow-up PNC. This PHC model complements the International Society for the Study of Hypertension in Pregnancy (ISSHP) recommendations for low resource settings [24] and resonates with new guidelines in South Africa [28].

This project has been pushing the agenda forward to provide better care for women on HDPs and $\mathrm{PE} / \mathrm{E}$ and has an expanded view of what is needed $[26,27,29,30]$. Ensuring services are available at lower levels provides opportunities to identify pre-eclampsia early, timely delivery and effective treatment for those with a severe form of the condition [14]. The results of the implementation research linked to this PHC model will be published as separate manuscripts. We are also considering reviewing the cost-effectiveness. If the mission of a public health system is to protect and improve pregnant women and their babies' healthcare, it will be imperative for Ministries of Health to act comprehensively in addressing the issues of $\mathrm{PE} / \mathrm{E}$ and MNH more broadly and specifically at PHC level $[15,18,24,30]$.

\section{Conclusion}

The PHC model described here uses PE/E as an entry to improve the quality of ANC and by extension the pregnancy continuum. Bringing preventive and treatment services nearer to where pregnant women live makes sense. The global community have recognized this by reendorsing PHC through the 2018 Astana commitment. Implementation research suggests that this PHC- PE/E model works and can improve the current and future health of women and infants.

\section{Abbreviations}

ANC: antenatal care; BP: blood pressure; CMWs: community midwives 
HDP: hypertensive disorders in pregnancy; MgSO4: magnesium sulphate; PE/ E: preeclampsia and eclampsia; PHC: primary health care; PNC: postnatal care; PPH: postpartum hemorrhage; SDG: Sustainable Development Goal

\section{Acknowledgements}

We wish to acknowledge the contribution of Ending Eclampsia colleagues and to Mary Ellen Stanton and Michelle Hindin for their input and review of an earlier version.

\section{Authors' contributions}

CEW conceived the idea and wrote the first draft, all authors critically reviewed the manuscript, read and approved the final version of the manuscript.

\section{Funding}

This work was supported by the US Agency for International Development (USAID), under the terms of cooperative agreement number [USAID APS- OAA-14-000048]. This article reflects the views of the authors and does not represent the views of the U.S. Government.

\section{Availability of data and materials}

Not applicable.

Ethics approval and consent to participate

Not applicable.

\section{Consent for publication}

Not applicable.

Competing interests

The authors declare that they have no competing interests.

\section{Author details}

${ }^{1}$ Population Council, Washington, DC, USA. 2Population Council, Dhaka, Bangladesh. ${ }^{3}$ Julius Centre for Health Sciences and Primary Care, UMC, Utrecht, The Netherlands. ${ }^{4}$ USAID, Washington DC, USA. 


\section{References}

1. Declaration of Astana. Astana: global conference on primary health care. Geneva: WHO; 2018. Available at https://www.who.int/primary-health/ conference-phc. Accessed 20 Feb 2019.

2. World Health Organization, UNICEF, UNFPA, the World Bank, United Nations Population Division. Trends in maternal mortality: 1990-2013. Geneva: Switzerland WHO; 2014. Available at. http://apps.who.int/iris/ bitstream/10665/112682/2/9789241507226_eng.pdf Accessed 28 Dec 2018.

3. United Nations Inter-Agency Group for Child Mortality Estimation (UN IGME). Levels \& trends in child mortality: report. New York: United Nations; 2018. Available at https://www.un.org/en/development/desa/population/

4. publications/mortality/child-mortality-report-2018.asp Accessed 20 Apr 2019.

5. Lawn JE, Blencowe $\mathrm{H}$, Waiswa $\mathrm{P}$, et al. Stillbirths: rates, risk factors, and acceleration towards 2030. Lancet. 2016;387(10018):587-603.

6. Say L, Chou D, Gemmill A, Tunçalp Ö, Moller AB, Daniels JD, et al. Global causes of maternal death: a WHO systematic analysis. Lancet Glob Health. 2014;2(6):323-33.

7. Salam RA, Das JK, Ali A, Bhaumik S, Lassi ZS. Diagnosis \& management of preeclampsia in community settings in low and middle-income countries. J Family Med Prim Care. 2015;4(4):501-6.

8. von Dadelszen P, Firoz T, Donnay F, et al. Preeclampsia in low- and middle- income countries-health services lessons learned from the PRE-EMPT (PRE- EclampsiaEclampsia Monitoring, Prevention and Treatment) project. J Obstet Gynaecol Can. 2012;34(10):917-26.

9. Pronyk PM, Nemser B, Maliqi B, for the UNCoLSC Technical Resource Teams, UN Agency Leads, and UNCoLSC Monitoring and Evaluation Advisory Group, et al. The UN Commission on Life Saving Commodities 3 years on: global progress update and results of a multi-country assessment. Lancet Glob Health. 2016; 4:276-86. 
10. Prata N, Bell S, Weidert K. Prevention of postpartum hemorrhage in low- resource settings: current perspectives. Int J Women's Health. 2013; 5:737-52. https://doi.org/10.2147/IJWH.S51661.

11. Warren CE, Hossain SM, et al. Landscape analysis of pre-eclampsia/eclampsia in Bangladesh report. New York: Population Council; 2016. Available at http://www.endingeclampsia.org/wp-content/uploads/2017/04/FINAL-

LandcapeAnalysis-Bangladesh-USAID-GA31-EndingEclampsia-Mar2016.pdf Accessed 30 Nov 2018.

12. Warren CE, Ishaku SM et al, Landscape analysis of pre-eclampsia/eclampsia in Nigeria report. New York: Population Council; 2016. Available at. http:// www.endingeclampsia.org/wp-content/uploads/2017/04/EndingEclampsiaLandscapeReport-Nigeria.pdf Accessed 30 Nov 2018.

13. Mir AM, Shaikh S, et al. Landscape report on pre-eclampsia/eclampsia in Pakistan report. New York: Population Council; 2016. Available at https:// www.popcouncil.org/uploads/pdfs/2016RH_LandscapingAnalysisPakistan. pdf. Accessed 2 Feb 2020.

14. World Health Organization (WHO). Recommendations on antenatal care for a positive pregnancy experience. Geneva: WHO; 2016. Available at https:// www.who.int/reproductivehealth/publications/maternal_perinatal_health/ ancpositive-pregnancy-experience/en/. Accessed 29 Dec 2018.

15. Hodgins S. Pre-eclampsia as underlying cause for perinatal deaths: time for action. Glob Health Sci Pract. 2015;3(4):525-7. https://doi.org/10.9745/GHSP- D-15-00350 Published 2015 Dec 17.

16. Magpie Trial Collaborative Group. Do women with pre-eclampsia, and their babies, benefit from magnesium sulphate? The Magpie Trial: a randomized placebo-controlled trial. Lancet. 2002;359(9321):1877-90.

17. World Health Organization (WHO). Recommendations for prevention and treatment of pre-eclampsia and eclampsia. Geneva: WHO; 2011. Available at: 
https://apps.who.int/iris/bitstream/handle/10665/44703/9789241548335_ eng.pdf. Accessed 29 Dec 2018.

18. World Health Organization (WHO). Model list essential medicines 18th list. Geneva: WHO; 2013. Available at https://www.who.int/medicines/ publications/essentialmedicines/en. Accessed 29 Nov 2018.

19. Smith JM, Lowe RF, Fullerton J, et al. An integrative review of the side effects related to the use of magnesium sulfate for preeclampsia and eclampsia management. BMC Pregnancy Childbirth. 2013; 13:34 Available at . http://www.biomedcentral.com/1471$\underline{2393 / 13 / 34}$.

20. Firoz Sanghvi H, Merialdi M, von Dadelszen P. Pre-eclampsia in low- and middle-income countries. Best Pract Res Clin Obstet Gynaecol. 2011; 25:537- 48.

21. Shamsuddin L, Nahar K, Nasrin B, Nahar S, et al. Use of parenteral magnesium sulphate in eclampsia and severe pre-eclampsia cases in a rural set up of Bangladesh. Bangladesh Med Res Counc Bull. 2005;31(2):75-82.

22. World Health Organization (WHO). Recommendations: optimizing health worker roles to improve access to key maternal and newborn health interventions through task shifting. Geneva: WHO; 2012. Available at https:// apps.who.int/iris/bitstream/handle/10665/250796/9789241549912-eng. pdf?sequence=1. Accessed 30 Nov 2018.

23. Brown M, Magee LA, Kenny LC, on behalf of the International Society for the Study of Hypertension in Pregnancy (ISSHP), et al. Hypertensive disorders of pregnancy: ISSHP classification, diagnosis, and management recommendations for international practice. Hypertension. 2018; 72:24-43.

24. Salam RA, Qureshi RN, Sheikh S, et al. Potential for task-sharing to Lady Health Workers for identification and emergency management of pre- eclampsia at community level in Pakistan. Reprod Health. 2016;13(Suppl 2): 107. Published 2016 Sep 30. https://doi.org/10.1186/s12978-016-0214-0.

25. Fujioka A, and Smith J. Prevention and management of postpartum hemorrhage and pre-eclampsia/eclampsia: national programs in selected USAID program-supported 
countries: Maternal and Child Health Integrated Program (MCHIP) global status reports. 2011. Available at: http://resources. jhpiego.org/resources/prevention-andmanagement-postpartum- hemorrhage-and-pre-eclampsia/eclampsia-national Accessed Dec 20, 2019.

26. Obstetrical and Gynecological Society of Bangladesh (OGSB), et al. Operationalizing the proposed national protocol for the prevention and management of severe preeclampsia and eclampsia patients using loading dose of magnesium sulphate at community level in Bangladesh. New York: Population Council. 2014. Available at https://www.popcouncil.org/research/

results?keywords=magnesium+sulphate\&resources=yes\&projects Accessed Feb 2020.

27. Pooja S, Nwala E, McClair T, Ishaku S, Warren CE. Engaging community women's groups to improve maternal health care delivery in cross river, Nigeria: implementation research report. New York: Population Council; 2019. Available at https://www.popcouncil.org/uploads/pdfs/2019RH_EE-

CommunityWomensGroupsCRS.pdf. Accessed 20 Jan 2020.

28. Ali $M$, et al. Expanding services to detect, manage, and prevent pre- eclampsia and eclampsia in Tando Allahyar District of Sindh Province, Pakistan implementation research report. New York: Population Council; 2019. Available at https://www.popcouncil.org/uploads/pdfs/2019RH_EE-

ExpandingServicesPakistan.pdf. Accessed 20 Jan 2020.

29. Moodley J, et al. Hypertensive disorders in pregnancy: 2019 national guideline. S Afr Med J. 109:9 ISSN 2078-5135. Available at: http://www.samj. org.za/index.php/samj/article/view/12723. Accessed 20 Jan 2020.

30. Nwala E, Anaba U, Sripad P, Ishaku SM, Warren CE. Feasibility and acceptability of community health extension workers to identify and treat hypertension associated with pregnancy. Implementation research report. New York: Population Council; 2019. Available at https://www.popcouncil. org/uploads/pdfs/2019RH_EECHEWsAntiHypertensives.pdf. Accessed 15 Jan 2020. 
31. Hossain S, Roy S, Sultana K, Warren CE. Assessing the effect of a primary health care intervention for improving pre-eclampsia and eclampsia knowledge and practice in Bangladesh. New York: Population Council; 2019. Available at http://www.endingeclampsia.org/wp-content/uploads/2019/10/ Report-BangladeshUSAID-Ending-Eclampsia-Final-Study-Report_2019.pdf Accessed 21 Jan 2020. 


\section{Chapter 9}

\section{Discussion}

\section{Enhancing research capacity to improve HDP-related health outcomes in LMICs: needs for NGOs-academia collaboration}

\section{Introduction}

In the preceding chapters, the chronic health burden posed by hypertensive disorders in pregnancy (HDP) have been examined. From this study and earlier evidence, HDP have been shown to be associated with risks of persistent (chronic) hypertension, cardiovascular disorders (ischemic heart diseases and cerebrovascular accident), acute and chronic kidney diseases, metabolic syndrome, and potentially type II diabetes. Despite the sustained high burden of HDP in sub-Saharan Africa, most of the current evidence comes from high-income countries (HICs) ${ }^{1,2}$. In general, while LMICs account for $92 \%$ of global diseases burden, only $10 \%$ of global research funding (and the actual conduct of research) goes to these regions, thereby creating inequality in research efforts, the so-called '10/90' gap². Despite growing efforts to correct this disproportional allocation of research resources, not much has changed ${ }^{1}$.

Over the past decades, enormous efforts have gone into improving access to quality health service deliveries in LMICs through the activities of International Non-governmental Organizations (INGOs) ${ }^{1}$. For INGOs with interest in maternal health services, the focus has been mainly on optimizing prepartum and intrapartum outcomes; with postpartum consequences of pregnancy and delivery on women's health often not adequately addressed ${ }^{3}$. This is a missed opportunity to promote lifelong cardiovascular and metabolic health of women at relatively young age. As critical as antepartum and intrapartum periods are, two-thirds of the $99 \%$ of global maternal death that occur in LMICs are postpartum in timing, mainly during puerperium. ${ }^{3}$. In addition, responding to health research needs of this population is also not a priority for many INGOs, with a few exceptions ${ }^{3}$. In instances where research is of interest, the capacities to generate valid evidence are limited ${ }^{4}$. Academic research institutions with capacity in research are 
not widely present in many LMICs, where the need for a locally-led research effort is crucial4,5. Lack of it creates a scenario of huge inequity in health research between the two settings.

Although of recent there has been an expansion in NGOs-led implementation science programs in many LMICs, they are mainly motivated by the need to bridging the know-do-gaps rather than providing answers to emerging health research questions based on implementation research principles ${ }^{6}$. Without rigorous research methods, concepts and methods of current implementation science programs in LMICs cannot be applied to achieve widespread health impact $^{7}$, and consequently, opportunities for continuous learning are, once again, not fully realized.

Prominent among the reasons why neither the routine INGOs service delivery models nor the current implementation programs adequately provides quality research outputs are identified as challenges hampering quality research outputs in LMICs and are discussed further below. The implication is that the bulk of NGOs-derived evidence only ends up as program reports for donors' need without enjoying scientific dissemination and contributing to the improvement in health care delivery outcomes and impact. One report indicated only $45 \%$ of evaluation results were shared publicly ${ }^{8}$. To bridge research gaps in LMICs settings, collaboration between INGOs and local academic research institutions is crucial.

\section{Challenges amid opportunities for research through INGOs program in LMICS}

Myriads of challenges, operating individually or in concert, hamper the utility of NGO-derived data in research. These include unskilled personnel/weak health system, lack of objective outcomes data and conflict of interest between donors, implementers, and researchers ${ }^{1,6,9}$.

Many NGO's health-related projects are implemented via public sector (Public-Private Partnership - PPP) for cost-effectiveness and sustainability with varying degree of success ${ }^{1}$. In most cases, however, services are provided either by local staff employed by NGOs or by the existing public health care providers. In both situations, personnel lack essential research skills or research intent is not considered ab initio or the facilities are not strengthened to support research activities ${ }^{1,9}$. In many NGOs-led and implementation programs, although monitoring and evaluation is always integral, the what? (in terms of outcomes and impact), the how? and the 
when? to monitor and evaluate programs are (too) often not well-defined at inception of health interventions ${ }^{6}$. A recent trend among donors is simultaneous engagement of Third-Party Monitors (TPMs) or professional evaluators to work along with program implementers with the view to improving evaluation outcomes. However, motives are mainly on adherence and independence of evaluation findings rather than asking pertinent scientific questions ${ }^{8,9}$. In addition, TPMs come into projects often too late to collect relevant quality data. Less than $25 \%$ of TPMs engage program implementers in planning, implementing and interpretating of results ${ }^{8}$. Another drawback with INGOs data is the tendency to focus predominantly on process indicators (inputs and outputs) with less emphasis on health outcomes and impact due to (un)justifiable belief that health impact and outcomes result from multiple complex pathways involving numerous stakeholders operating simultaneously, therefore, not appropriate to be claimed by one project or organization ${ }^{13}$. Donors' interests are largely responsible for INGOs' over-emphasis on process indicators ${ }^{10}$. Unfortunately, policy-changing research derives their values in proving that interventions result in improved health outcomes. These conflicting expectations between development work and mainstream science further render predominantly NGOs-derived data inadequate for use to answer emerging questions in health care research. Furthermore, NGOs do not routinely capture and report the unintended effects of their program interventions.

Finally, because NGOs' interventions are donors-driven, there is often a conflict of interests in term of what donors want and what science expects; donors frequently ask for indicators of accountability, coverage and value for money ${ }^{1,10}$. In contrast, science requires high-quality data based on rigorous methodologies that validly explain existing and or emerging health challenges. Since NGOs are accountable to their donors, they focus on service delivery coverage indicators as opposed to generating valid scientific evidence. In fact, many NGOs are of the view that proposals with a focus on research outputs would not be funded by donors ${ }^{10}$. Hence, the increasing tendency among NGOs to focus on service delivery indicators.

\section{Promoting NGOs/academia collaboration in research}

Given NGOs' superior comparative advantages over academic institutions in extensive coverage and access to LMICs settings where research gaps exist, enabling environments that should set 
the stage for NGO-academia collaboration in research is already in place. Over the past years, the emphasis has been on promoting collaboration between NGOs and public sector health systems in poor-resource countries on one hand ${ }^{1}$ and 'openness' and 'transparency' of research findings on the other hand ${ }^{11}$. While this is, undoubtedly, yielding progress, there has not been proportionate growth in our ability to answer key research questions on the health of this population. Promoting and building NGOs-academia research collaboration in LMICs could potentially advance our guess to realizing this and subsequently reduce research inequity in global health. Such collaboration should start very early in projects conceptualization for it to be considered a product of teamwork.

One way to achieve seamless NGO-academia collaboration, for example, is building a culture of developing joint proposals in response to donors' calls that serve both academic and program's interest. At this stage, suitable professional evaluators can be invited to contribute their expertise in designing rigorous evaluation methodologies. How subsequent implementation and evaluation of projects are conducted can be agreed upon by the collaborating parties at inception through improved communication ${ }^{16}$, but this is of lesser significance provided the initial project designs are collaborative with objectively well-defined interventions and outcome measures that are relevant to both parties.

Governing issues between collaborating parties are potential hindrances to a smooth team's work. This can be addressed once a mutually developed project protocol (based on trust, transparency and respect) is agreed upon ${ }^{12}$, including development of detailed validated data capturing tools. For instance, project implementation and routine evaluation can be carried out by partner NGOs and professional evaluators, while academic collaborators are given unlimited access to the collected data for subsequent relevant analyses and interpretation. Alternatively, academic institutions can be given direct access to project sites for direct data collection as appropriate. This approach should lead to the collection of pertinent scientifically relevant information that minimizes measurement biases. In essence, a key to harmonious and productive collaboration is transparency in communication throughout the process ${ }^{12}$. 
An unintended but foreseen unhealthy consequence of joint NGO-academia response to a proposal is the potential to generate competing interest in the distribution of financial resources and ownership of results among the collaborating parties ${ }^{12}$. This can be avoided with opened and fair agreement at the start. For example, academic institutions must not necessarily be grants recipients as partners on health programs. Grants could go to NGOs, but postgraduate students from academic institutions should be given opportunities to access project sites and collect scientifically relevant information as already captured in the original protocol jointly conceptualized. This should create a win-win situation between the parties in the sense that while NGOs receive funding and implement in line with donors' expectations, academia use their platforms to collect sound scientifically relevant data at no costs. This approach should also minimize potential conflict with donors' needs. Information that is relevant for donor's needs is summarized and reported by NGOs and professional evaluators while academic researchers retrieve information that is relevant for scientific consumption.

Ultimately, such collaboration would promote quality evidence generation and dissemination on pertinent global health challenges in both high-income and LMICs, thereby reducing inequality and improving quality in research outcomes. While academia is better placed to provide sound theoretical, methodological, technical expertise and capacity to advance knowledge through scientific publication, NGOs align research efforts with local needs and political realities, communicate research findings to policymakers and facilitate community engagement and participation in the development, implementation, and uptake of research ${ }^{15}$

\section{HDP-related health research as priority}

Over the next decades, the burden of HDP to women's health in sub-Saharan Africa is predicted to increase exponentially due to rising rates of obesity, essential hypertension and advanced maternal age $\mathrm{e}^{17-19}$. These factors by themselves and in conjunction with HDP are likely to increase the burden of cardiometabolic disorders in LMICs settings leading to rising premature deaths and disability-adjusted life years (DALYs) lost ${ }^{18}$. However, most of the current evidence of poorer health outcomes associated with HDP come from studies in HICs. This thesis is one of few examples of original research that looked at the future health outcomes of women after HDP- 
complicated pregnancies. Although the initial ideas and designs of the work came from the INGO setting, further reviews on its technical and methodological components by experts in academia ensured relevant scientifically beneficial insight were derived, and are being communicated in relevant fora in order to contribute to new knowledge in this emerging field.

Given the foregoing, and while a general call for narrowing research inequalities between the HICs and LMICs is timely and reasonable, researching into the intermediate- and long-term health outcomes associated with HDPs in LMICs should be given a pride of place in this collaboration.

\section{Recommended next steps}

INGOs implementing HDP-related programs in LMICs should collect and record accurate blood pressure measurements using validated devices starting in the first trimester of pregnancy. For women with HDP, measurement and documentation of blood pressure should continue in the postpartum period until when the blood pressure is normalized, or clients referred for specialist care. In cases of referral, efforts should be made to track blood pressure recordings until resolution of high blood pressure.

INGOs operating in LMICS on HDP-related programs should collaborate with local Obstetricians and researchers in academia to develop monitoring and evaluation tools that are responsive to research needs in relation to the association between any HDP-type with future risks of cardiovascular and metabolic disorders. Examples of research priorities in this respect are as recommended by the International Federation of Gynecologists and Obstetricians (FIGO) ${ }^{20}$, the National Institute for Health and Care Excellence (NICE) ${ }^{21}$, ISSHP22 and the World Health Organization $^{23 .}$

When women with HDPs are seen during antenatal care, efforts should be made to ensure, as much as possible, information about their pre-conceptional health is obtained accurately and documented for subsequent estimation of morbidities to be valid. 


\section{References}

1. Olivia B, Martin E, Siw Ca, Magnus F, Birger C. Forsberg. Collaboration between nongovernmental organizations and public services in health a qualitative case study from rural Ecuador. Citation: Glob Health Action 2016, 9: 32237 http://dx.doi.org/10.3402/gha.v9.32237

2. Global Forum for Health Research. The 10/90 report on health research 2000. Geneva: Global Forum for Health Research, 2000.

3. Étienne VL, Malgorzata M, Maria VZ, Abdul G, Daniela Z, Igor Karp. Inequities in postnatal care in low- and middle-income countries: a systematic review and meta-analysis. Bulletin of the World Health Organization 2015;93:259-270G. doi: http://dx.doi.org/10.2471/BLT.14.140996

4. Franzen SRP, Chandler C, Lang T. Health research capacity development in low- and middle-income countries: reality or rhetoric? A systematic meta-narrative review of the qualitative literature. BMJ Open 2017;7: e012332. doi:10.1136/bmjopen-2016-012332

5. Dye C, Boerma T, Evans D et al. The World Health Report: research for universal health coverage. Geneva: World Health Organization, 2013.

6. Alonge $\mathrm{O}$, Rodriguez $\mathrm{DC}$, Brandes $\mathrm{N}$ et al. How is implementation research applied to advance health in low-income and middle-income countries? BMJ Glob Health 2019;4: e001257. doi:10.1136/bmjgh-2018-001257

7. Sanders $D$, Haines $A$. Implementation research is needed to achieve international health goals. PLoS Med 2006;3: e186.

8. Amy A, Prithi T, Fay T. The Value of our Evaluations: Assessing Spending and Quality. Hewlett Foundation. February 2018.

9. Elias $S$, Julia $S$, András $D$, Camille $H$. The use of third-party monitoring in insecure contexts: Lessons from Afghanistan, Somalia and Syria. Secure Access in volatile environment; Resource paper October 2016. Retrieved September 11, 2020 from https://www.gppi.net/media/SAVE_2016 The use of third party monitoring in insecure contexts.pdf 
10. Gooding K, Newell JN, Emmel N. Capacity to conduct health research among NGOs in Malawi: Diverse strengths, needs and opportunities for development. PLOS ONE (2018) 13(7): e0198721. https://doi.org/10.1371/journal.pone.0198721

11. Storeng KT, Abimbola $S$, Balabanova $D$ et al. Action to protect the independence and integrity of global health research. BMJ Global Health 2019;4: e001746. doi:10.1136/ bmjgh-2019-001746

12. Catherine O, Matthew RH, Valéry R. NGO-researcher partnerships in global health research: benefits, challenges, and approaches that promote success, Development in Practice, (2016) 26:4, 444-455, DOI: 10.1080/09614524.2016.1164122

13. Donald C, Tamara C. Successful Interventions, Positive Outcomes: Understanding the Impact of Social Determinants of Health. Retrieved September 29, 2020 from https://www.ajmc.com/view/successful-interventions-positive-outcomesunderstanding-the-impact-of-social-determinants-of-health

14. Zachariah R, Ford N, Draguez B, Yun O, Reida T. Conducting Operational Research within a Non-Governmental Organization: The Example of Médecins Sans Frontières. International Health; 2010. 2: 1-8.

15. Delisle H, Roberts JH, Munro M, Jones L, Gyorkos TW. The Role of NGOs in Global Health Research for Development. Health Research Policy and Systems; 2005. 3: 3.

16. Stephen, C, Daibes I. Defining Features of the Practice of Global Health Research: An Examination of 14 Global Health Research Teams. Global Health Action; 2010. 3: 5188.

17. Gregory AR, Mark DH, Andrew EM et al. Global and Regional Patterns in Cardiovascular Mortality from 1990 to 2013. Circulation. 2015; 132:1667-1678. DOI: 10.1161/CIRCULATIONAHA.114.008720.)

18. Suzan H, Wim G, Milena P. Trends in cardiovascular diseases and associated risks in subSaharan Africa: a review of the evidence for Ghana, Nigeria, South Africa, Sudan and Tanzania, The Aging Male, 2019. 22:3, 169-176, DOI: 10.1080/13685538.2019.1582621

19. Van LLM, Yahner M, Bakamjian L. Women's growing desire to limit births in sub-Saharan Africa: meeting the challenge. Glob Health Sci Pract. 2013;1(1):97-107. http://dx.doi.org/10.9745/GHSP-D-12-00036 
20. Laura AM, Peter VD, William S, Mathew M. The FIGO textbook of pregnancy hypertension: An Evidence-based guide to monitoring, prevention and management. 2016; ISBN: 9780-9927545-5-6

21. National Institute for Health and Care Excellence (NICE) guideline. Hypertension in pregnancy: diagnosis and management. 2019. www.nice.org.uk/guidance/ng133

22. Mark AB, Laura AM, Louise $C K$ et al. Hypertensive Disorders of Pregnancy: ISSHP Classification, Diagnosis, and Management Recommendations for International Practice. Hypertension. 2018; 72:24-43. doi: 10.1161/HYPERTENSIONAHA.117.10803.

23. WHO recommendations for Prevention and treatment of pre-eclampsia and eclampsia. World Health Organization, 20 Avenue Appia, 1211 Geneva 27, Switzerland. ISBN 97892 41548335. 


\section{Appendix}

Summary

Samenvatting

Contributing authors

Acknowledgement

Curriculum vitae

List of Publications 
Appendix/summary

\section{Summary}

Hypertensive disorders in pregnancy is the second leading cause of maternal mortality in lowand middle-income countries after postpartum hemorrhage. It is the leading cause of deaths and morbidity in referral care setting in Nigeria. Despite the progress of the past decades in reducing maternal deaths in low- and middle-income setting, most of the reduction were results of improvement in the prevention and management of postpartum hemorrhage. Preventing deaths and complications from HDP are proving very challenging. This is because, unlike postpartum hemorrhage, prediction of complications from HDP is not always easy especially in primary and secondary health care facilities where majority of deliveries take place. In addition to the immediate pregnancy-associated complications of HDP, women whose pregnancies were complicated by HDP stand additional risks of long-term negative health outcomes such as cardiovascular, renal and metabolic disorders. All these inter-connections have not been fully elucidated in many low- and middle-income countries.

This thesis starts with looking at gaps in the care of women with hypertensive disorders during pregnancy, delivery and in the immediate postpartum period in Nigeria. Short- and intermediateterm medical conditions associated with hypertensive disorders in pregnancy were then evaluated. Subsequently, the roles of communities, where women live, and the potential contribution women could play in improving their care and outcomes were investigated.

In part I, quality of care for women with hypertensive disorders in pregnancy was reviewed. Chapter 2, the knowledge and skills of antenatal care providers and the capacity of the health system to detect and manage hypertensive disorders among pregnant women in Nigeria was evaluated. All domains of quality except provider interpersonal skills scored below $55 \%$. The lowest overall scores were observed in provider knowledge (49.9\%) and provider technical skill (47.7\%). PHCs performed significantly worse than hospitals in all elements of quality except for provider interpersonal skills. Provider knowledge was significantly associated with their level of designation. Chapter 3 assessed level of compliance and adherence to standard guidelines in postpartum management of women with HDP across tertiary hospitals in Nigeria. This also 
revealed paucity in the level of adherence and provision of life-saving interventions. In general, level of adherence to guidelines on postpartum management of HDP in tertiary hospitals in Nigeria is poor. It is recommended that institutionalization of these guidelines be prioritized and linked to the entire continuum of care from pre-conception, pregnancy, childbirth through longer term postpartum care.

In part II of this thesis, the prevalence, incidence and determinants of common medical disorders associated with prior HDP were investigated. Chapter $\mathbf{4}$ assessed the prevalence of persistent hypertension up to one year in women with HDP in a low resource setting and determined associated risk factors. Of the 410 women enrolled, 278 were followed up to 1 year after delivery (follow up rate 68\%). Among women diagnosed with gestational hypertension and preeclampsia/eclampsia, $22.3 \%(95 \% \mathrm{Cl} ; 8.3-36.3)$ and $62.1 \%$ (95\% Cl; 52.5 - 71.9), respectively, had persistent hypertension at 6 months and this remained similar at one year $22.3 \%(95 \% \mathrm{Cl}$; 5.6 - 54.4) and 61.2\% (95\% Cl; 40.6 - 77.8), respectively). Maternal age and body mass index were significant risk factors for persistent hypertension at one year after delivery [aORs = $1.07 /$ year (95\% Cl; $1.02-1.13)$ and $1.06 / \mathrm{kg} / \mathrm{m}^{2}$ (95\% Cl; 1.01-1.10)], respectively.

Chapter 5 studied the association of metabolic syndrome with history of hypertensive disorders in pregnancy. The prevalence of metabolic syndrome among those with HDPs were $17.4 \%$ ( 71 of 407 ), $8.7 \%$ (23 of 263), 4.7\% (11 of 232) and 6.1\% (17 of 278), at delivery, nine weeks, six months and one year postpartum, respectively. The incidence rate for metabolic syndrome in HDPs versus normotensive at one year were, respectively, 57.5/1000 person years $(95 \% \mathrm{Cl} ; 35.8$ - 92.6). and $16.9 / 1000$ person years (95\%Cl; $2.4-118.3)$, with incidence rate ratio of $3.4 / 1000$ person years. Only parity significantly predicted the presence of metabolic syndrome for women with HDPs at one year in the multivariable logistic analysis.

Chapter 6 investigated if the reported association between previous HDP (especially preeclampsia) and chronic kidney diseases (defined by decreased eGFR $<60 \mathrm{~mL} / \mathrm{min} / 1.73 \mathrm{~m}^{2}$ lasting for $\geq$ three months) in high-income setting applies to Nigeria's women. Within 24 hours of delivery, nine weeks and six months postpartum, women with HDP were more likely to have a decreased eGFR compared to their normotensive counterparts $(12 \%, 5.7 \%, 4.3 \%$ versus $0 \%, 2 \%$ 
and $2.4 \%$ respectively). The prevalence of CKD in HDP at six months and one year postpartum was $6.1 \%$ and $7.6 \%$, respectively, as opposed to zero prevalence in the normotensive cohort for the corresponding periods. Only maternal age independently predicted occurrence of decreased eGFR at six months postpartum.

In part III, the thesis focuses on various community-based interventions in the management of hypertensive disorders in pregnancy that could improve the health and wellbeing of women, their babies and families during and after pregnancy. Therefore Chapter $\mathbf{7}$ explored survivor perceptions of pre-eclampsia and eclampsia in Nigeria through the health belief model. It was found that many of the beliefs, attitudes, knowledge and behaviors of women are consistent across the country, with some variation between the north and south. Women's perceived susceptibility and threat of health complications during pregnancy and childbirth, including preeclampsia and eclampsia, influence care-seeking behaviors. Moderating influences include acquisition of knowledge of causes and signs of pre- eclampsia, the quality of patient-provider antenatal care interactions, and supportive discussions and care seeking- enabling decisions with families and communities. These cues to action mitigate perceived mobility, financial, mistrust, and contextual barriers to seeking timely care and promote the benefits of maternal and newborn survival and greater confidence in and access to the health system.

Chapter 8 recommends a primary health care model for managing pre-eclampsia and eclampsia that should adopted and scale up in low- and middle- income countries. The PHC $\mathrm{PE} / \mathrm{E}$ model displays the linkages and opportunities to prevent and treat $\mathrm{PE} / \mathrm{E}$ in a simplified way; however, there are numerous interlinking factors, angles, and critical points to consider including leadership, policies and protocols; relevant medicines and commodities, ongoing capacity building strategies at lower levels and understanding what women and their communities want for safe pregnancies.

In conclusion, this thesis recommends strict vigilance on all women at risk of developing HDP or with confirmed diagnosis along entire maternity continuum; pre-conceptional, antenatal, intranatal and postnatal periods. For successful management outcomes, all stakeholders (women, the communities, health care providers, policy-makers) should be carried along in all aspects that 
improve care and survival of women with HDP. All stakeholders should be educated that effects of HDP are not limited to during pregnancy and childbirth but potentially life-long. In order to accelerate research around HDP in low- and middle-income countries, a collaboration between local/international non-governmental organization and academic research institutions is highly recommended. 


\section{Samenvatting}

Hypertensieve aandoeningen tijdens de zwangerschap (HDP's) zijn na postpartumbloedingen de meest voorkomende oorzaak van maternale mortaliteit in landen met lage en gemiddelde inkomens. HDP's zijn de voornaamste oorzaak van sterfte en morbiditeit in de referente zorg in Nigeria. Er is de afgelopen decennia vooruitgang geboekt wat betreft het terugdringen van maternale sterfte in settings met lage en gemiddelde inkomens, maar de daling is vooral het gevolg van verbeteringen bij de preventie en behandeling van postpartumbloedingen. De preventie van sterfte en complicaties als gevolg van HDP's blijkt grote uitdagingen met zich mee te brengen. Dit komt doordat complicaties van HDP's, anders dan postpartumbloedingen, niet altijd eenvoudig te voorspellen zijn, met name in primaire en secundaire zorginstellingen, waar de meeste bevallingen plaatsvinden. Vrouwen van wie de zwangerschap gecompliceerd werd door HDP's lopen naast de onmiddellijke zwangerschapsgerelateerde complicaties van HDP's bijkomende risico's op langdurige negatieve gezondheidsuitkomsten zoals cardiovasculaire aandoeningen, nieraandoeningen en stofwisselingsstoornissen. De onderlinge verbanden hiertussen in veel landen met lage en gemiddelde inkomens zijn niet helemaal duidelijk.

In dit proefschrift wordt ten eerste gekeken naar leemten in de zorg voor vrouwen met hypertensieve aandoeningen tijdens de zwangerschap, de bevalling en de onmiddellijke postpartumperiode in Nigeria. Daarna worden kort- en middellangdurende medische aandoeningen beoordeeld die in verband worden gebracht met hypertensieve aandoeningen tijdens de zwangerschap. Vervolgens wordt de rol van de gemeenschappen waarin de vrouwen leven onderzocht, alsook de mogelijke bijdrage die vrouwen kunnen leveren aan de verbetering van hun zorg en van de uitkomsten.

In deel I wordt de kwaliteit van de zorg voor vrouwen met hypertensieve aandoeningen tijdens de zwangerschap beoordeeld. In hoofdstuk 2 wordt gekeken naar de kennis en vaardigheden van prenatale zorgaanbieders en het vermogen van de gezondheidszorg om hypertensieve aandoeningen bij zwangere vrouwen in Nigeria te kunnen vaststellen en behandelen. Behalve voor de interpersoonlijke vaardigheden van de zorgaanbieder was de score voor alle kwaliteitsdomeinen lager dan 55\%. De totaalscores waren het laagst voor de kennis van de 
zorgaanbieder $(49,9 \%)$ en de technische vaardigheden van de zorgaanbieder $(47,7 \%)$. Primaire zorginstellingen scoorden op alle onderdelen behalve interpersoonlijke vaardigheden van de zorgaanbieder aanzienlijk lager dan ziekenhuizen. De kennis van de zorgaanbieder stond in sterke mate in verband met het niveau van zijn of haar functie. In hoofdstuk $\mathbf{3}$ wordt beoordeeld in hoeverre bij de postpartumbehandeling van vrouwen met HDP's in tertiaire ziekenhuizen in Nigeria de standaardrichtsnoeren werden nageleefd. Hieruit komt tevens naar voren dat levensreddende interventies in geringe mate werden nageleefd en toegepast. In het algemeen was in tertiaire ziekenhuizen in Nigeria sprake van een slechte naleving van de richtsnoeren met betrekking tot de postpartumbehandeling van HDP's. Aanbevolen wordt om prioriteit te geven aan institutionalisering van deze richtsnoeren en deze institutionalisering te koppelen aan het gehele zorgcontinuüm vanaf de preconceptie, de zwangerschap en de bevalling tot en met de postpartumzorg voor de langere termijn.

In deel II van dit proefschrift worden de prevalentie, de incidentie en de determinanten van vaak voorkomende medische aandoeningen in verband met eerdere HDP's onderzocht. In hoofdstuk 4 wordt de prevalentie van aanhoudende hypertensie tot één jaar beoordeeld bij vrouwen met HDP's in een lage-inkomenssetting en worden hiermee in verband staande risicofactoren vastgesteld. Van de 410 aan het onderzoek deelnemende vrouwen werden er 278 tot één jaar na de bevalling gevolgd (follow-up-percentage 68\%). Bij vrouwen met de diagnose zwangerschapsgerelateerde hypertensie en pre-eclampsie/eclampsie, was na zes maanden bij respectievelijk 22,3\% (95\% Bl; 8,3 - 36,3) en 62,1\% (95\% BI; 52,5 - 71,9) sprake van aanhoudende hypertensie en was de situatie na één jaar vergelijkbaar (respectievelijk $22,3 \%(95 \% \mathrm{Bl} ; 5,6-54,4)$ en $61,2 \%$ (95\% BI; 40,6 - 77,8)). Maternale leeftijd en body mass index waren één jaar na de bevalling significante risicofactoren voor aanhoudende hypertensie [respectievelijk aOR's = 1,07/jaar (95\% Bl; $1,02-1,13)$ en 1,06/kg/m² $(95 \% \mathrm{Bl} ; 1,01-1,10)$ ].

In hoofdstuk 5 wordt gekeken naar het verband tussen metabool syndroom en een voorgeschiedenis van hypertensieve aandoeningen tijdens de zwangerschap. De prevalentie van metabool syndroom onder vrouwen met HDP's was bij de bevalling en negen weken, zes maanden en één jaar postpartum respectievelijk 17,4\% (71 van de 407), 8,7\% (23 van de 263), $4,7 \%$ (11 van de 232 ) en 6,1\% (17 van de 278 ). Het incidentiepercentage voor metabool syndroom 
bij HDP's ten opzichte van normotensief na één jaar was respectievelijk 57,5/1000 personenjaren $(95 \% \mathrm{Bl} ; 35,8$ - 92,6) en 16,9/1000 personenjaren (95\% Bl; $2,4-118,3$ ), met een relatief incidentierisico van 3,4/1000 personenjaren. Alleen pariteit voorspelde significant de aanwezigheid van metabool syndroom voor vrouwen met HDP's na één jaar in de multivariabele logistische analyse.

In hoofdstuk 6 wordt onderzocht of het gemelde verband tussen eerdere HDP's (met name preeclampsie) en chronische nierziekten (gedefinieerd door verlaagde eGFR $<60 \mathrm{ml} / \mathrm{min} / 1,73 \mathrm{~m}^{2}$ gedurende $\geq$ drie maanden) in een hoge-inkomenssetting van toepassing is op vrouwen in Nigeria. Binnen 24 uur na de bevalling en negen weken en zes maanden postpartum was het risico op een verlaagde eGFR bij vrouwen met HDP's groter dan bij normotensieve vrouwen (respectievelijk 12\%, 5,7\%, 4,3\% t.o.v. 0\%, 2\% en 2,4\%). De prevalentie van CKD bij HDP's zes maanden en één jaar postpartum was respectievelijk $6,1 \%$ en $7,6 \%$ ten opzichte van een prevalentie van nul in het normotensieve cohort voor de overeenkomstige perioden. Alleen maternale leeftijd voorspelde onafhankelijk het optreden van een verlaagde eGFR zes maanden postpartum.

Deel III van het proefschrift is toegespitst op diverse interventies in gemeenschapsomgevingen bij de behandeling van hypertensieve aandoeningen tijdens de zwangerschap waarmee de gezondheid en het welzijn van vrouwen, hun pasgeboren kind en hun gezin tijdens en na de zwangerschap kan worden verbeterd. Om die reden worden in hoofdstuk 7 percepties van overlevenden van pre-eclampsie en eclampsie in Nigeria verkend aan de hand van het gezondheidsgeloofsmodel (Health Belief Model, HBM). Er werd vastgesteld dat een groot deel van de overtuigingen, attitudes, kennis en gedrag van vrouwen door het hele land consistent was, met enige variatie tussen noord en zuid. De door vrouwen waargenomen vatbaarheid en dreigende gezondheidsproblemen tijdens de zwangerschap en de bevalling, waaronder preeclampsie en eclampsie, zijn van invloed op het zorgzoekend gedrag. Matigende invloeden zijn onder andere de verwerving van kennis over oorzaken en tekenen van pre-eclampsie, de kwaliteit van patiënt-zorgverlenerinteracties in de prenatale zorg en ondersteunende gesprekken met en zorgzoekende en -faciliterende beslissingen aangaande gezinnen en gemeenschappen. Deze impulsen tot actie verkleinen waargenomen belemmeringen in verband 
met mobiliteit, financiën en wantrouwen en contextuele belemmeringen om tijdig zorg te zoeken en vergroten de overleving van moeders en pasgeborenen alsook het vertrouwen in en de toegang tot de gezondheidszorg.

In hoofdstuk 8 wordt een aanbeveling gedaan voor de invoering en opschaling van een model voor de behandeling van pre-eclampsie en eclampsie (PE/E-model) in primaire zorginstellingen in landen met lage en gemiddelde inkomens. In het PE/E-model voor primaire zorginstellingen worden de verbanden en kansen met betrekking tot de preventie en behandeling van PE/E op vereenvoudigde wijze weergegeven; er moeten echter talrijke verbindende factoren, invalshoeken en kritieke punten in aanmerking worden genomen, onder andere met betrekking tot leiderschap, beleid en protocollen, relevante geneesmiddelen en producten, lopende capaciteitsvormende strategieën op lagere niveaus en de wensen van vrouwen en de gemeenschappen waarin ze leven op het gebied van veilige zwangerschappen.

Tot slot wordt in dit proefschrift een aanbeveling gedaan voor nauwlettende bewaking van alle vrouwen met een risico op of een bevestigde diagnose van HDP's in het gehele zorgcontinuüm rond de zwangerschap (tijdens de preconceptionele, prenatale, intranatale en postnatale periode). Voor succesvolle behandeluitkomsten dienen alle belanghebbenden (vrouwen, de gemeenschappen, zorgaanbieders, beleidsmakers) bij alle aspecten die tot verbetering van de zorg en overleving van vrouwen met HDP's leiden, te worden betrokken. Alle belanghebbenden dienen te leren dat de effecten van HDP's niet beperkt blijven tot de periode tijdens de zwangerschap en de bevalling maar mogelijk levenslange gevolgen hebben. Om het onderzoek rond HDP's in landen met lage en gemiddelde inkomens te versnellen wordt samenwerking tussen lokale/internationale niet-gouvernementele organisaties en academisch onderzoeksinstellingen ten zeerste aanbevolen. 


\section{Contributing authors}

Angela, Salomon

Dalla Lana School of Public Health, Toronto, Canada

Karen, R. Kirk

Population Council, One Dag Hammarskjöld Plaza, 3rd Floor, New York, NY 10017, USA

Charlotte, E. Warren

Population Council, 4301 Connecticut Avenue NW Suite 280, Washington, DC 20008, USA

Lawal, Oyeneyin

University of Medical Sciences Teaching Hospital, Ondo, Ondo State, Nigeria

Onyebuchi, Azubuike

Federal Teaching Hospital, Abakaliki, Ebonyi State, Nigeria

Innocent, Agbo

Population Council, Abuja, Nigeria

Lamaran, Dattijo

Abubakar Tafawa Balewa University Teaching Hospital, Bauchi, Bauchi State, Nigeria

Aminu, Baffah

Abubakar Tafawa Balewa University Teaching Hospital, Bauchi, Bauchi State, Nigeria

Gbenga, Kayode

Julius Global Health, Julius Center for Health Sciences and Primary Care, University Medical Center Utrecht, Utrecht University, The Netherlands and Institute of Human Virology, Abuja, Nigeria

Olorunfemi, Owa

Mother and Child Hospital, Akure, Nigeria

Patience, Odusolu

University of Calabar Teaching Hospital, Calabar, Cross River State, Nigeria 
Karima, Tunau

Usman DanFodio University Teaching Hospital, Sokoto, Sokoto State, Nigeria

Jamilu, Tukur

Bayero University/Aminu Kano Teaching Hospital Kano, Kano State, Nigeria

Hannifa, Abubakar

Muhammad Abdullahi Wase Teaching Hospital, Kano, Kano State, Nigeria

Musa, Abdulkarim

Federal Medical Center, Lokoja, Kogi State, Nigeria

Arie, Franx

Erasmus Medical Center, University Medical Center, Rotterdam, The Netherlands

Diederick, Grobbee

Julius Global Health, Julius Center for Health Sciences and Primary Care, University Medical Center Utrecht, Utrecht University, The Netherlands

Joyce, Browne

Julius Global Health, Julius Center for Health Sciences and Primary Care, University Medical Center Utrecht, Utrecht University, The Netherlands

Pooja, Sripad

Population Council, 4301 Connecticut Avenue NW Suite 280, Washington, DC 20008, USA

Gloria, Adoyi

Population Council, No. 16 Mafemi Crescent, Utako District, Abuja, Nigeria

Amy, Dempsey

Population Council, 4301 Connecticut Avenue NW Suite 280, Washington, DC 20008, USA 
Sharif Mohammed, Ismail Hossain

Population Council, Dhaka, Bangladesh

Deborah, Armbruster

United States Agency for International Development, Washington DC, USA.

Emily, Hillman

United States Agency for International Development, Washington DC, USA.

Khadijat, A Oboirien

Usmanu DanFodio University Teaching Hospital, Sokoto, Sokoto State, Nigeria

Kerstin-Klipstein, Grobusch

Julius Global Health, Julius Center for Health Sciences and Primary Care, University Medical Center Utrecht, Utrecht University, The Netherlands and Division of Epidemiology and Biostatistics, School of Public Health, Faculty of Health Sciences, University of the Witwatersrand, Johannesburg, South Africa

Timothy Olusegun, Olanrewaju

University of Ilorin Teaching Hospital, Ilorin, Kwara State, Nigeria 
Appendix/acknowledgements

\section{Acknowledgments}

At the conference of the International Society for the Study of Hypertension in Pregnancy (ISSHP) on October 2015 In Sao Paulo Brazil, I was privileged to be a co-panelist with Dr Joyce L. Browne. Like an Angel of mercy, Joyce quickly recognized that my area of interest perfectly aligns with that of the Global Health Program at the Julius Center for Health Science and Primary Care, University Medical Center Utrecht, the Netherlands. This connection triggered chains of events leading to my enrollment in both the PhD and the MSc Epidemiology programs. Apart from being a game-changer, Joyce, I appreciate the enormous amount of your time, patience and support you invested on me daily from the beginning to the end. Thank you so much.

Because I discontinued with earlier PhD opportunity in the United States to focus on completing my residency training six years before meeting Joyce, I was too reluctant to accept this challenge until after I discussed with Dr Charlotte Warren, then, one of my supervisors at the Population Council. Her push, encouragement, materials and technical support was crucial to my eventual enrollment into the program. Thank you, Charlotte.

My subsequent meetings with Professors, Rick Grobbee and Arie Franx and Dr Kerstin KlipsteinGrobusch assured me that I was in good and trusted hands. You were always warm and welcoming, caring and energizing. By introducing me to Dr Gbenga Kayode as a Local supervisor, you already provided me with a platform to succeed and flourish. Thank you, Dr Kayode for coping with my nuisance that knew no limit: during official hours, weekends and holidays.

My appreciation goes to the members of the Ending Eclampsia project who worked tirelessly to make sure the project was a success. Although the list is endless, the following deserve a special mention: Rahat Ara Nur, Kanij Sultana and Gloria Adoyi (who were part of the Ending Eclampsia team at the ISSHP conference in Sao Paulo), Sharif Mohammed Hussein, Innocent Agbo, Emmanuel Nwala, Pooja Sripad, Karen Kirk, Amy Dempsy, Angela Salomon, Charity Ndwiga, 
Deborah Armbruster, Mary Ellen Stanton, and Emily Hillman. Thank you all for the roles you played in my success.

To all the co-investigators at the various locations of this project (Professors Jamilu Tukur and Karima Tunau, Drs. Lamaran Dattijo, Lawal Oyeniyin, Musa Abdulkarim, Onyebuchi Azuibuike, Patience Odusolu and Owa Olorunfemi), thank you for your partnership and support. The support and cooperation of all the staff and clients from your facilities is deeply appreciated.

During this difficult period, I derived immense support and goodwill from Babatunde Ahonsi, John Townsend, Saumya RamaRao, Chi-Chi Undue, Harriet Birungi and Michelli Hindin; extension of the care and mentorship I used to receive during my time with Population Council. Your contribution to my development and success will forever be remembered.

To all my colleagues on the global Women's Integrated Sexual Health (WISH) team - Rachel Cullen, Caroline Guinard, Kaitlin Keane, Laura Nurse, Heidi Brown, Susan Camara, Megan McLaren, Karen Penrose-Theis, Gurvir Deol, Ellen Clancy, Ellora Howie, Sarah Palmer, Alhaji Sheriff and Lisa Abwa-Renard - your daily and weekly encouragement and goodwill provided the courage and energy I needed to keep working on my thesis as the COVID-19 pandemic raged on. Your approval of a time for me to work from Utrecht in the course of this program will always be remembered. I learned one aspect of humanity working with you.

Finally, I would like to acknowledge the roles and support provided by many of my colleagues in the global health program and at the Julius Center who at different times made enormous sacrifice to make the completion work of this and of the MSc Epidemiology program a reality. It is almost impossible to mention everyone but permit to identify a few. Thank you, Marcus Rijken, Melanie' Peroz, Giene de Vries, Jestke Hartman, Maud, Anna Marza Florensa, Klaartje Olde Loohuis, Timothy Olanrewaju, Kwame Adu-Bonsaffoh, Natasha Housseine, Engelbert Nonterah, Daniel Boteng, Wout and Josephine Rees. 


\section{Curriculum vitae}

Salisu Ishaku is a scientist and expert in maternal and child health and family planning with extensive experience as a clinician, researcher, health policy champion and public health advocate. He is a physician with a background in Obstetrics and Gynecology with over 19 years of experience. He has worked across all levels of health care system in Nigeria from implementing programs aimed at preventing deaths from childhood illnesses, maternal mortality and morbidity and agitating for policies that improve the lives of women and children.

The first six years of his career was dedicated to clinical practice, a major part of it as a registrar in Obstetrics and Gynecology providing pre-natal care, family planning services, gynecology cancer screening, intra-partum and postpartum care services and surgical treatment of obstetric complications such as obstetric fistulas and genital tract lacerations. As a public health practitioner, he has worked as a service consultant providing technical assistant to local organizations to expand access to modern contraception in northern Nigeria and supported secondary health facilities across Nigeria to implement the continuum of care for postpartum hemorrhage prevention and management, which included active management of third stage of labor and use on non-pneumatic anti-shock garment for the treatment of hypotensive shocks following obstetric hemorrhage.

Salisu is one of the leading public health experts to introduce magnesium sulphate as prophylaxis and treatment of severe pre-eclampsia in secondary facilities in Kano state of Nigeria and his work with lower cadre providers at primary health centers to administer loading dose of magnesium sulphate paved the way for the change in national task-shifting policy to allow this cadre to administer the loading dose of magnesium before referral to higher centers. His work on pre-eclampsia/eclampsia in Nigeria has attracted international attention including further grants from USAID under the Ending Eclampsia project to replicate these successes in several states in southern Nigeria and other international locations including Bangladesh, Ethiopia, Kenya and Pakistan. He led the Nigeria's arm of the Ending Eclampsia project and supported its implementation in other countries. 
He served as a temporary consultant for the World Health Organization/Merck for Mothers collaboration on pre-eclampsia and eclampsia in Geneva, Switzerland in 2013 and participated at the joint WHO/UNICEF/UNFPA experts' consultation on the Definition and Coverage Measurement of Skilled Attendance at birth in June 2016 in New York City, United States of America.

He has also worked extensively using both qualitative and quantitative research to assess the knowledge, attitudes and practice of health care providers and policymakers around Emergency Contraception (EC) in Nigeria. This led to a series of policy changes including listing of EC on the Essential Drug List in the country. He has collaborated on many occasions with the World Health Organization and International Consortium for Emergency Contraception (ICEC) on expert panels, local and international workshops and conferences on EC.

He was a part of the team of Population Council researchers that implemented the Bill and Melinda Gates Foundation's funded acceptability study of Progesterone Vaginal Ring in Kenya, Nigeria and Senegal. The successful conduct of the study and associated researches (e.g. willingness to pay for the ring study, market segmentation) paved the way for additional support from USAID to register and introduce the ring in sub-Saharan Africa. Salisu led the Nigeria arm of the study and supported in other countries.

He was also one of the Principal Investigators on the Evidence project - USAID-funded- to conduct implementation science for family planning and reproductive health in many countries including Nigeria. In Nigeria, he led an operational research to assess and determine the feasibility of Patent Medicine Vendors (PMVs) to provide all forms of services around progestogen-based injectable contraceptives, including Sayana Press. This was a policy-changing intervention given the roles of PMVs in Nigeria's health system. This model is being replicated in many countries in Africa and Asia. 
He was one of the Population Council's experts that worked in nine countries toward generating evidence to eliminate Female Genital Mutilation/Cutting (FGM/C) in one generation with funding from the United Kingdom Department for International Development (DfID) in collaboration with UN joint program (UNICEF/UNFPA) and Girl's Generation. In collaboration with Engender Health (the Fistula Care Plus project), Salisu worked on a global team to generate evidence to eliminate barriers hindering women with Obstetrics Fistula from assessing curative care in Nigeria and Uganda. He coordinated the activities of five service-providing NGOs, including Marie Stopes International, Rotary International, Pathfinder International and Planned Parenthood Federation of Nigeria to increase access to Levonorgestrel Intra-uterine System (LNG IUS) to poor women, in a program runs by the International Contraceptive Access (ICA) Foundation.

Salisu currently work as Nigeria's partnership lead for the Women Integrated Sexual Health (WISH) project, an FCDO-funded project operating in about thirty countries in Africa and Asia.

He previously worked as a teaching assistant in Leadership and Communication Skills and as a research assistant at the Center for Global Health, Tulane University to develop a new training curriculum for medical schools in Ethiopia.

He obtained his medical degree from Ahmadu Bello University, Zaria, Nigeria and an MPH degree in global maternal and child health from the School of Public Health and Tropical Medicine, Tulane University, New Orleans, USA, He obtained two fellowship awards from Ford Foundation and Compton Foundation. 


\section{List of publications}

Charlotte E. Warren, Sharif Mohammed Ismail Hossain, Salisu Ishaku, Deborah Armbruster, Emily Hillman. A primary health care model for managing pre-eclampsia and eclampsia in low- and middle- income countries. Reproductive Health (2020) 17:46; https://doi.org/10.1186/s12978020-0897-0

Ekechi Okereke, Salisu Mohammed Ishaku, Godwin Unumeri, Bello Mohammed, Babatunde Ahonsi. Reducing maternal and newborn mortality in Nigeria-a qualitative study of stakeholders' perceptions about the performance of community health workers and the introduction of community midwifery at primary healthcare level. Human Resources for Health (2019) 17:102. https://doi.org/10.1186/s12960-019-0430-0

Pooja Sripad, Karen Kirk, Gloria Adoyi, Amy Dempsey, Salisu Ishaku, Charlotte E. Warren. Exploring survivor perceptions of preeclampsia and eclampsia in Nigeria through the health belief model. BMC Pregnancy and Childbirth (2019) 19:431. https://doi.org/10.1186/s12884-019-2582$\underline{2}$

Angela Salomon, Salisu Ishaku, Karen R. Kirk, Charlotte E. Warren. Detecting and managing hypertensive disorders in pregnancy: a cross-sectional analysis of the quality of antenatal care in Nigeria. BMC Health Services Research (2019) 19:411. https://doi.org/10.1186/s12913-0194217-8

Sara Chace Dwyer, Aparna Jain, Salisu Mohammed Ishaku, Faizah Tosin Okunade, Chiamaka Uzomba, Adedamola Adebayo, Elizabeth Tobey. The effect of job aids on knowledge retention among Patent and Proprietary Medicine Vendors trained to administer injectable contraceptives: longitudinal results from implementation science in Nigeria. BMC Public Health (2019) 19:1362. https://doi.org/10.1186/s12889-019-7668-2

Salisu M Ishaku, Nafissatou Diop, Babacar Mané, Wilson Liambila, Saumya RamaRao, Heather Clark, Harriet Birungi, Godwin Unumeri, Francis Obare. Introducing the Progesterone Vaginal Ring (PVR) as a New Option for Postpartum Family Planning in Sub-Saharan Africa: Insights from Engagements with Stakeholders. African Journal of Reproductive Health 2018; 22[2]:68-75. DOI: 10.29063/ajrh2018/v22i2.7.

Saumya RamaRao, Francis Obare, Salisu Ishaku, Babacar Mané, Heather Clark, Wilson Liambila, Godwin Unumeri, Harriet Birungi, Nafissatou Diop, Deepa Rajamani, and John Townsend. DoWomen Find the Progesterone Vaginal Ring Acceptable? Findings from Kenya, Nigeria, and Senegal. Studies in Family Planning 49(1), 2018. 
Mark A. Brown, Laura A. Magee, Louise C. Kenny, S. Ananth Karumanchi, Fergus P McCarthy, Shigeru Saito, David R. Hall, Charlotte E. Warren, Gloria Adoyi, Salisu Ishaku. The hypertensive disorders of pregnancy: ISSHP classification, diagnosis \& management recommendations for international practice. Pregnancy Hypertension 13 (2018) 291-310. https://doi.org/10.1016/i.preghy.2018.05.004

Sara Chace Dwyer, Salisu Mohammed Ishaku, Faizah Okunade, Laura Reichenbach, Aparna Jain. Feasibility of patent and proprietary medicine vendor provision of injectable contraceptives: preliminary results from implementation science research in Oyo and Nasarawa, Nigeria. Contraception (2018), https://doi.org/10.1016/j.contraception.2018.08.015

Jamilu Tukur, Mohammed Salisu Ishaku, Babatunde Ahonsi, Ayodeji Babatunde Oginni, Gloria Adoyi. Training-of-trainers of nurses and midwives as a strategy for the reduction of eclampsia-related maternal mortality in Nigeria. Sahel Medical Journal / 2016 / Vol 19 | Issue 2; ISSN 1118-8561.

Salisu Ishaku Mohammed, Babatunde Ahonsi, Ayodeji Babatunde Oginni, Jamilu Tukur, Gloria Adoyi. Obstetric knowledge of nurse educators in Nigeria: Levels, regional differentials and their implications for maternal health delivery. Health Education Journal 1-10 2015. DOI: 10.1177/0017896915571763.

Godwin Unumeri, Salisu Ishaku, Babatunde Ahonsi, Ayodeji Oginni. Contraceptive Use and Its Socio-economic Determinants among Women in North-East and North-West Regions of Nigeria: A Comparative Analysis. African Population Studies Vol. 29, No. 2, 2015.

Saumya RamaRao, Salisu Ishaku, Wilson Liambila, Babacar Mane. Enhancing contraceptive choice for postpartum women in sub-Saharan Africa with the progesterone vaginal ring: a review of the evidence. Journal of Contraception 2015:6 117-123. http://dx.doi.org/10.2147/OAJC.S55033

Salisu Ishaku, Babatunde Ahonsi, Jamilu Tukur, Oginni Ayodeji. Attrition from care after the critical phase of severe pre-eclampsia and eclampsia: Insights from an intervention with magnesium sulphate in a primary care setting in northern Nigeria. Health Journal; Vol.5, No.9, 1461-1466 (2013); http://dx.doi.org/10.4236/health.2013.59199

Ekechi Okereke, Babatunde Ahonsi, Jamilu Tukur, Salisu Mohammed Ishaku and Ayodeji Babatunde Oginni. Benefits of using magnesium sulphate (MgSO4) for eclampsia management and maternal mortality reduction: lessons from Kano State in Northern Nigeria. BMC Research Notes 2012, 5:421; http://www.biomedcentral.com/1756-0500/5/421 
Jamilu Tukur, Babatunde Ahonsi, Salisu Mohammed Ishaku, Idowu Araoyinbo, Ekechi Okereke, Ayodeji Oginni Babatunde. Maternal and Fetal Outcomes After Introduction of Magnesium Sulphate for Treatment of Preeclampsia and Eclampsia in Selected Secondary Facilities: A LowCost Intervention. Maternal and Child Health Journal. DOI 10.1007/s10995-012-1105-9 